\title{
Planar Laser Induced Iodine Fluorescence Investigation of Mach 5 Jet-Jet and Jet-Jet-Surface Interactions
}

\author{
Presented to \\ The Faculty of the \\ School of Engineering and Applied Science \\ University of Virginia \\ In Partial Fufillment \\ of the Requirements for the Degree \\ Doctorate of Philosophy in Mechanical and Aerospace Engineering \\ by \\ David Barnes \\ November 7, 2017 \\ On my honor as a University student, on this assignment I have neither given nor \\ received unauthorized aid as defined by the Honor Guidelines for Papers in Science, \\ Technology, and Society Courses. \\ (Full signature) \\ James McDaniel (Signature) \\ Christopher Goyne (Signature) \\ Houston Wood (Signature) \\ Daniel Quinn (Signature) \\ Zongli Lin (Signature) \\ (Advisor) \\ (Committee Chair) \\ (Committee Member) \\ (Committee Member) \\ (Out-of-Department \\ Committee Member)
}

Approved

Approved

Approved

Approved

Approved 


\section{Abstract}

During approach maneuvers with the International Space Station (ISS), an autonomous spacecraft performed an emergency procedure in order to decrease the closing rate as the spacecraft approached the space station. NASA Johnson Space Center had performed Direct Simulation Monte Carlo (DSMC) fluid dynamics simulations in order to certify the maneuver in the vicinity of the ISS. When the spacecraft performed the maneuver however, it resulted in exterior damage to the International Space Station.

Following the flight incident, the University of Virginia's Aerospace Research Laboratory engaged in high speed fluids research in an effort to ascertain the root cause of the differences in the computational model and real-world fluid dynamics observed during the HTV maneuvers. Planar Laser Induced Iodine Fluorescence was utilized as the spectroscopic method with which to qualitatively and quantitatively investigate the complex hypersonic flowfield. Qualitative broadband visualizations were collected to visually classify each flowfield, as well as determine quantitative flowfield geometry measurements. Visualizations were collected for numerous configurations including varying the number of exhausting jets (between two and four exhausting jets), varying the separation between neighboring jets, and for a variety of impingement standoff distances. Along with providing qualitative images of the flowfield interactions, quantitative flowfield density ratios were measured. The quantitative density ratio experiments were conducted for a smaller subset of experimental configurations. The combination of the qualitative images, geometry data, and density ratio data provides significant experimental insight into the complex jet-jet and jetjet-surface interactions.

Results from the research presented herein led to three major conclusions. First, by increasing the jet spacing the neighboring jet interaction decreased, as expected. Second, the removal of two exhausting jets led to a 5 times smaller mixing percentage, which means mixing was enhanced by the two additional jets. Finally, for both the four jet and two jet flow configurations, the introduction of the impingement surface to the flow yielded an approximately $70 \%$ increase in total mixing percentage, which means there was an approximately $70 \%$ increase to the neighboring jet interactions. The data collected will be applied directly to the refinement of the NASA DSMC algorithm. The additional refinement of the analysis algorithm will result in changes to spaceflight operating procedures surrounding the approach and docking / capture process with the International Space Station. 


\section{ACKnowledgements}

First, I would like to thank my adviser, Jim McDaniel, for taking me on as a graduate student at the ARL. Even though I came to the ARL from 'unique' circumstances, I have always felt included and supported. Jim has always acted as an incredible mentor, and I thank him for his help and guidance. I also want to thank Barry for introducing me to Jim, and whose perfect timing resulted in my being able to participate in this research. I also need to thank Houston Wood for working with me at ROMAC in my early days at the University. I would also like to express a huge thank you to Josh. Josh imparted all of the lab wisdom, and a little bit of the magic voodoo, to me when he was completing his experiments and dissertation. He was always more than happy to include me in his experiments, and made me feel an equal when helping on his project or working on the beginnings of my own, even when I felt overwhelmed and lost during that first year at the ARL. Along this same line, I would like to thank all of the undergraduate interns who provided valuable assistance in the lab and for putting up with my random tangential ideas.

I also need to thank the most important people in my personal life, my family. First, I have to thank my wife for her never-ending support and patience while I spent longer than expected completing this degree. She was positive when I needed support, but also strong and focused when I needed course adjustments. Without her at my side, this degree wouldn't mean nearly as much as it does. My mother and father were instrumental in motivating me in school, and for helping cultivate my life-long interest in learning. My mother was always there for me, lending emotional (as well as occasional financial) assistance while I worked on this research. I have very strong memories of instances in my childhood spent with my father that directly led to pursuing engineering as a career, and for those experiences I am forever grateful, and I miss him deeply. And last, but by no means least, I would like to thank my brother for helping to keep me sane while I worked through the degree.

I would like to thank NASA Johnson Space Center, and specifically Forrest Lumpkin, for the grant support and for the opportunity to conduct this research. Finally, thank you to everyone whom I may have forgotten to mention, without the myriad

of support and assistance I received, I never would have completed this research and written this thesis. 


\section{Contents}

Abstract

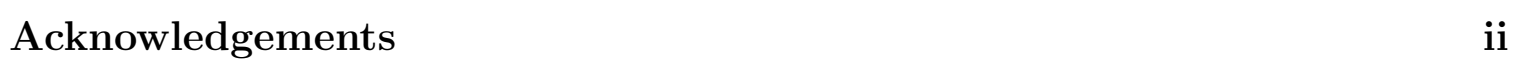

Contents $\quad$ iii

$\begin{array}{ll}\text { List of Tables } & \text { v }\end{array}$

List of Figures $\quad$ vi

List of Variables $\quad$ ix

$\begin{array}{lll}\mathbf{1} \text { Introduction } & \mathbf{1}\end{array}$

1.1 Research Objectives . . . . . . . . . . . . . . . . . . 4

$\begin{array}{llr}2 & \text { Background } & 6\end{array}$

2.1 New Research in Context . . . . . . . . . . . . . . . . . . . . . . 18

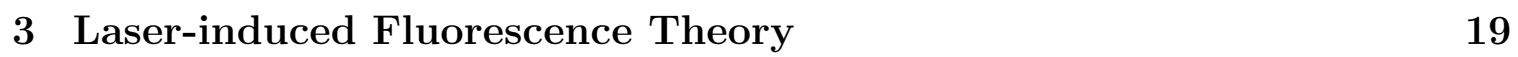

3.1 Iodine Model and Governing Rate Equation . . . . . . . . . . . . . . 20

3.1 .1 Molecular Iodine Energy Transitions . . . . . . . . . . . . . . 20

3.1.2 Governing Rate Equation . . . . . . . . . . . . . . . . . . . 23

3.1 .3 Boltzmann Population Fraction . . . . . . . . . . . . . . . . . 25

$3.1 .4 \quad$ Stern-Volmer Factor - Fluorescence Efficiency . . . . . . . . . 29

3.1 .5 Voigt Function - Line Shape . . . . . . . . . . . . . . . . . . . 29

3.2 Narrowband Measurements . . . . . . . . . . . . . . . . . . . . . . . . 33

3.2.1 Velocity Measurement . . . . . . . . . . . . . . . . . . 34

3.3 Broadband Measurements . . . . . . . . . . . . . . . . . . . . . 35

3.3 .1 Visualization . . . . . . . . . . . . . . . . . . . 36

3.3 .2 Density Ratio . . . . . . . . . . . . . . . . . . . . . . . . . . 40

4 Design of Experiment and Experimental Procedures 42

4.1 Experimental Setup . . . . . . . . . . . . . . . . . . . . . . . . . . . . . . . 42

4.1 .1 Hypersonic Wind Tunnel . . . . . . . . . . . . . . . . . . . . . 44

$4.1 .2 \quad$ Optical Systems . . . . . . . . . . . . . . . . . . . . . . . . . . 47

$4.1 .3 \quad$ Argon-ion Laser . . . . . . . . . . . . . . . . . . . . . . . . . . 48

$4.1 .4 \quad$ Charge-Coupled Device Camera . . . . . . . . . . . . . . . . . 49 
4.2 Design of Experiment . . . . . . . . . . . . . . . . . . . . . . . . . . . 49

4.2 .1 Model Mounting Design . . . . . . . . . . . . . . . . . . . 51

4.2 .2 Test Section Design . . . . . . . . . . . . . . . . . . . . 54

4.3 Experimental Procedure . . . . . . . . . . . . . . . . . . . . . 61

\begin{tabular}{|lll}
5 & Results - Broadband Visualization & $\mathbf{6 4}$
\end{tabular}

5.1 Disk 1 Qualitative Results . . . . . . . . . . . . . . . . . . . . . . . . 64

5.2 Disk 2 Qualitative Results . . . . . . . . . . . . . . . . . . . . . . . . . . 77

5.3 Disk 3 Qualitative Results . . . . . . . . . . . . . . . . . . . . 80

5.4 Flowfield Comparisons . . . . . . . . . . . . . . . . . . . . . . . 82

$5.5 \quad$ PLIIF Fluorescence Phenomenon . . . . . . . . . . . . . . . . . . . 88

6 Results - Quantitative Density Ratio 100

6.1 Broadband Density Ratio . . . . . . . . . . . . . . . . . . . 100

6.2 Density Ratio Contour Plots and Mixing Area . . . . . . . . . . . . . 101

7 Conclusions and Recommendations 121

7.1 Visualization and Density Ratio . . . . . . . . . . . . . . . . . . . . . 122

7.2 Research Objective 1 . . . . . . . . . . . . . . . . . . . . . 123

7.3 Research Objective 2 . . . . . . . . . . . . . . . . . . . . . . . . . 124

7.4 Impact of this Research. . . . . . . . . . . . . . . . . . . . . . . . . 127

7.5 Recommendations for Future Work . . . . . . . . . . . . . . . . . . . 128

\begin{tabular}{ll}
\hline Appendix A Data Summary Table & 130
\end{tabular}

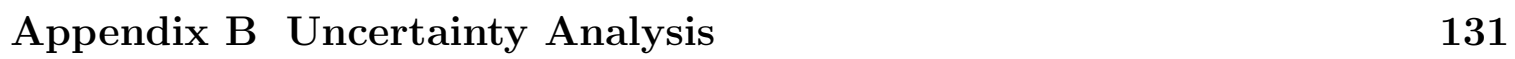

B.1 Uncertainty Analysis for Broadband Techniques . . . . . . . . . . . . 131

B.2 Uncertainty Analysis for Density Ratio . . . . . . . . . . . . . . . . . 133

\begin{tabular}{|lll}
\hline Appendix C Latvala \& Anderson Jet Boundary Prediction & 137
\end{tabular}

\begin{tabular}{|lll}
\hline Appendix D Iodine Model Matlab Code & 141
\end{tabular}

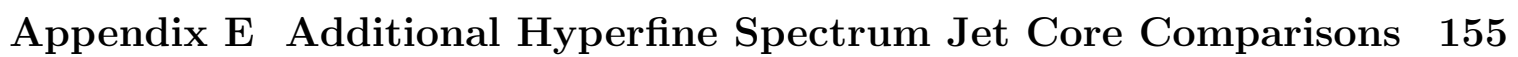

\begin{tabular}{ll}
\hline References & 169
\end{tabular} 


\section{LIST OF TABLES}

2.1 Table of mean free path ranges and $\mathrm{Kn} / \mathrm{mm}$ for various atmospheric pressure/vacuum ranges. . . . . . . . . . . . . . . . . . . . . 10

4.1 Pertinent physical dimensions. . . . . . . . . . . . . . . . . . . 55

$5.1 \quad$ Pertinent geometry measurements, in $\frac{x}{D}$. . . . . . . . . . . . . . . 84

5.2 Pertinent geometry measurements, in $\frac{x}{D}$. . . . . . . . . . . . 85

5.3 Pertinent geometry measurements, in $\frac{x}{D}$. . . . . . . . . . . . 88

A.1 Pertinent geometry measurements. . . . . . . . . . . . . . . . 130

A.2 Mixing Percentage Comparison. . . . . . . . . . . . . . . . . . . . . . 130 


\section{List OF FigURES}

1.1 HTV 2 Spacecraft. . . . . . . . . . . . . . . . . . . . 2

1.2 HTV Mach 5 Main Engine. . . . . . . . . . . . . . . . . . . 2

1.3 HTV ISS Mission Profile. . . . . . . . . . . . . . . . . . 3

1.4 NASA DSMC Simulation of Event. . . . . . . . . . . . . . . 4

$2.1 \quad$ Diagram of an under-expanded jet structure. . . . . . . . . . . 7

$2.2 \quad$ Cartesian coordinate system overlaid with cylindrical system. . . . . 16

$3.1 \quad$ Energy Level Diagram for the Diatomic Iodine Molecule. . . . . . . . 21

3.2 Diagram of Energy Transfer Mechanisms for Diatomic Iodine. . . . . 22

3.3 $\quad$ Boltzmann population fraction contribution from vibrational quantum

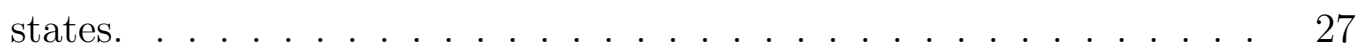

$3.4 \quad$ Boltzmann population fraction contribution from rotational quantum states. . . . . . . . . . . . . . . . . . . . 28

$3.5 \quad$ Hyperfine spectrum for P13/R15 overlapping transitions in diatomic

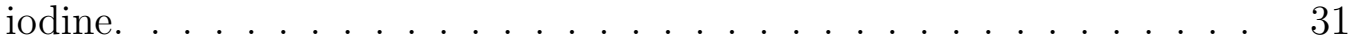

$3.6 \quad$ Overlay of $514.5 \mathrm{~nm}$ argon-ion gain profile and iodine absorption spec-

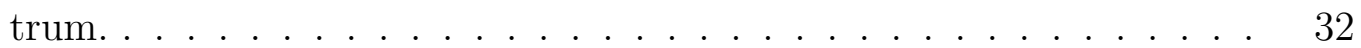

$3.7 \quad$ Static cell spectrum versus spectrum at a point in the flowfield used to determine Doppler shift. . . . . . . . . . . . . . . . . . . 35

3.8 Stern-Volmer Factor versus Mach Number. . . . . . . . . . . . . . 37

3.9 Boltzmann population fraction versus Mach number. . . . . . . . . 38

3.10 Product of Stern-Volmer Factor and Boltzmann Population Fraction versus Mach Number. . . . . . . . . . . . . . . . . . . . . . . . . . . . 39

4.1 Basic experimental layout. . . . . . . . . . . . . . . . . . . . . 43

4.2 Hypersonic test chamber and optics. $\ldots \ldots \ldots \ldots \ldots$

4.3 Test facility plumbing diagram. . . . . . . . . . . . . . . . . 46

4.4 Previous hypersonic test chamber configuration. . . . . . . . . . . 50

4.5 SolidWorks representation of the model design. . . . . . . . . . . . 51

4.6 Completed model design mounted in the test chamber. . . . . . . . . . 53

4.7 Completed experiment design with laser sheet projected on model disk.. 54

4.8 Designs for Nozzle Disk. $\ldots \ldots \ldots \ldots \ldots \ldots \ldots$

$4.9 \quad$ Jet Boundary Solution Method from Latvala and Anderson. . . . . . 57

4.10 Jet Interaction Indicated by Boundary Prediction for Nozzle Disk 1. . 57

4.11 Nozzle Disk 1 Experimental Interaction versus Prediction. . . . . . . 58

4.12 SolidWorks Exploded Model Assembly. . . . . . . . . . . . . . . 60 
4.13 As-machined Nozzle Disk $3 . \quad \ldots \ldots$. . . . . . . . . . . . 60

$5.1 \quad$ Visualization of Doppler Bias in Broadband Images. . . . . . . . . . . 66

5.2 Disk 1 - Four Jet Free Exhaust Fluorescence Plots. . . . . . . . . . . 68

5.3 Disk 1 - Four Jet Free 3.62" Impingement Fluorescence Plots. . . . . 70

5.4 Disk 1 - Four Jet Free 2.875" Impingement Fluorescence Plots. . . . . 71

5.5 Disk 1 - Four Jet Free 2.875" Impingement Fluorescence Plots. . . . . 72

5.6 Disk 1 - Two Jet Free Exhaust Fluorescence Plots. . . . . . . . . . . 74

5.7 Disk 1 - Two Jet, 3.62" Impingement Standoff Fluorescence Plots. . . 75

5.8 Disk 1 - Two Jet, 2.875" Impingement Standoff Fluorescence Plots. . 76

5.9 Disk 1 - Two Jet, 2.1875" Impingement Standoff Fluorescence Plots. . $\quad 77$

5.10 Disk 2 - Four Jet Free Exhaust. . . . . . . . . . . . . . . . . . 78

5.11 Disk 2 - Four Jet, 2.1875" Impingement Standoff Fluorescence Plots. 79

5.12 Disk 3 - Four Jet Free Exhaust Fluorescence Plots. . . . . . . . . . . 80

5.13 Disk 3 - Four Jet, 2.1875" Impingement Standoff Fluorescence Plots. 81

5.14 Comparison of Four Jet Free Exhaust Fluorescence Visualizations. . . 83

5.15 Comparison of Four Jet Free 2.1875" Standoff Visualizations. . . . . . 85

5.16 Comparison of Disk 1 Four and Two Jet Fluorescence Visualizations. 87

5.17 Fluorescence Contour Plot for a Single Jet, Free Exhaust. . . . . . . . 89

5.18 Predicted Absorption Spectrum Output from the Iodine Model. . . . 91

5.19 Ashkenas \& Sherman prediction vs. Centerline Fluorescence. . . . . . 93

5.20 Single Jet Contour Plot. . . . . . . . . . . . . . . . . . . . . 94

5.21 Overlay of Three Fluorescence Horizontal Cross-sections. . . . . . . . 95

5.22 Row 150, Comparison of Predicted Spectrum and Fluorescence Signal. 96

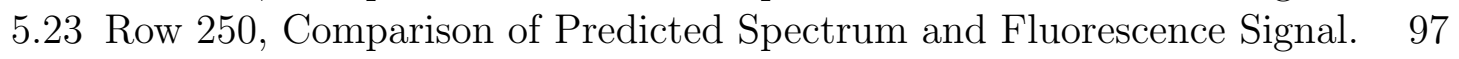

5.24 Row 350, Comparison of Predicted Spectrum and Fluorescence Signal. 98

6.1 SolidWorks Model for Density Ratio Experiments. . . . . . . . . . . . 100

6.2 Component Images for Density Ratio Calculation. . . . . . . . . . . . . 102

6.3 Example of Mixing Integral and Flow Integral Calculations for a Single Pixel Row. . . . . . . . . . . . . . . . . . . . . 104

6.4 Single Jet Seeded versus Density Ratio Contour Plots, for 4 jet, free

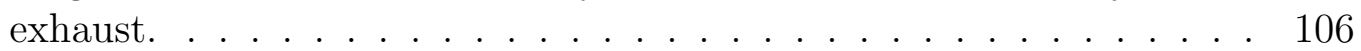

6.5 Density Ratio image - 4 Jet Free Exhaust. . . . . . . . . . . . . . . . 107

6.6 Mixing Percentage at each downstream pixel. . . . . . . . . . . . . . 109

6.7 Single Jet Seeded versus Density Ratio Contour Plots for 4 jet, 3.52" Standoff Distance. . . . . . . . . . . . . . . . . . . . . . 110

6.8 Density Ratio Image - 4 Jet, 3.52" Standoff. . . . . . . . . . . . . . . 111

6.9 Mixing Percentage at each downstream pixel. . . . . . . . . . . . . . 113

6.10 Single Jet Seeded Fluorescence versus Density Ratio Images for 2 jet free exhaust. . . . . . . . . . . . . . . . . . . . 114

6.11 Density Ratio Image - 2 Jet, Free Exhaust. . . . . . . . . . . . . . . . 115

6.12 Mixing Percentage at each downstream pixel. . . . . . . . . . . . . . 116

6.13 Single Jet Seeded Fluorescence versus Density Ratio Images for 2 Jets Impinging on a Surface at 2.54". . . . . . . . . . . . . . . . . . 117 
6.14 Density Ratio image - 2 Jet, 2.54" Standoff. . . . . . . . . . . . . . . 118

6.15 Mixing Percentage at each downstream pixel. . . . . . . . . . . . . . 119

E.1 Single jet contour plot, $P_{\text {ratio }}=19310 . \quad$. . . . . . . . . . . . . . . . 155

E.2 Overlay of Three Fluorescence Horizontal Cross-sections. . . . . . . . 156

E.3 Row 350, Comparison of Predicted Spectrum \& Fluorescence Signal. . 157

E.4 Row 250, Comparison of Predicted Spectrum \& Fluorescence Signal. . 158

E.5 Row 150, Comparison of Predicted Spectrum \& Fluorescence Signal. . 159

E.6 $\quad$ Single jet contour plot, $P_{\text {ratio }}=18170 . \ldots$. . . . . . . . . . . . . 160

E.7 Overlay of Three Fluorescence Horizontal Cross-sections. . . . . . . . . 161

E.8 Row 350, Comparison of Predicted Spectrum \& Fluorescence Signal. . 162

E.9 Row 250, Comparison of Predicted Spectrum \& Fluorescence Signal. . 163

E.10 Row 150, Comparison of Predicted Spectrum \& Fluorescence Signal. . 164 


\section{List OF VARIABLES}

$\begin{array}{ll}A & \text { Density ratio scaling constant } \\ a & \text { Speed of sound } \\ A_{21} & \text { Fluorescence emission rate, } s^{-1} \\ B & \text { Excited energy state } \\ b_{12} & \text { Rate of iodine excitatioin to B-state } \\ b_{21} & \text { Rate of iodine stimulated emission } \\ \mathrm{C} & \text { Fluorescence experimental constant } \\ \mathrm{c} & \text { Speed of light in a vacuum } \\ c_{q} & \text { Empirically determined quenching constant } \\ \mathrm{d} & \text { Particle hard shell diameter } \\ d_{j e t} \text { exit } & \text { Jet exit diameter } \\ f_{1} & \text { Fraction of molecules in rotational and vibrational quantum } \\ & \text { configurations, in X-state, corresponding to states resonant } \\ & \text { with the laser } \\ f_{2} & \text { Fraction of molecules in rotational and vibrational quantum } \\ & \text { configurations, in B-state, corresponding to states resonant } \\ f_{J}, & \text { with the laser } \\ f_{s} & \text { Population contribution of rotational quantum state } \\ f_{v \prime} & \text { Iodine seeding fraction } \\ f_{v, J} & \text { Population contribution of vibrational quantum state } \\ I & \text { Boltzmann population fraction } \\ J^{\prime \prime} & \text { Laser Intensity } \\ k_{b} & \text { Rotational quantum number } \\ K n & \text { Boltzmann constant } \\ L & \text { Knudsen number } \\ l_{j e t-j e t} & \text { Representative physical length scale } \\ M & \text { Center-to-center jet spacing } \\ m & \text { Mach number } \\ n & \text { Mass } \\ N_{1} & \text { Number density } \\ N_{2} & \text { Total population of available iodine in the X-state } \\ N_{d} & \text { Total population of available iodine in the B-state } \\ p & \text { Predicted number density } \\ p_{b} & \text { Pressure } \\ p_{o} & \text { Back pressure } \\ P_{r a t i o} & \text { Plenum pressure } \\ & \text { Pressure ratio } \\ & \end{array}$




$\begin{array}{ll}P_{t} & \text { Total pressure } \\ Q_{t o t a l} & \text { Total energy flux } \\ Q_{21} & \text { Collisional quenching rate } \\ Q_{23} & \text { Pre-dissociation rate of diatomic iodine } \\ S_{f} & \text { Fluorescence signal intensity } \\ T & \text { Temperature } \\ T_{t} & \text { Total temperature } \\ u & \text { Velocity component in the x-direction } \\ V & \text { Voigt lineshape function } \\ v & \text { Velocity component in the y-direction } \\ v^{\prime \prime} & \text { Vibrational quantum number } \\ w & \text { Flow width normalized by jet exit diameter } \\ X & \text { Ground energy state } \\ \frac{x}{D} & \text { Downstream distance normalized by jet exit diameter } \\ G r e e s y m b o l s & \\ \gamma & \text { Specific heat ratio } \\ \lambda & \text { Mean free path or laser wavelength } \\ \mu & \text { Mach angle } \\ \Phi & \text { Velocity potential } \\ \rho & \text { Density } \\ \rho_{t} & \text { Density of gas in the plenum } \\ \sigma & \text { Degeneracy of rotational quantum state } \\ \theta & \text { Angle between velocity vector and coordinate system } \\ \theta_{n} & \text { Cirection } \\ \theta_{\text {rot }} & \text { Cozzle exit angle } \\ \Delta v & \text { Deturacteristic rotational temperature } \\ \Delta v_{D} & \text { Doppler broadening term (Doppler linewidth) } \\ \Delta v_{o} & \end{array}$




\section{Chapter 1}

\section{INTRODUCTION}

Spaceflight in the 21st century, especially support for a persistent manned presence in space, has become a multinational effort consisting of support from Russia, Japan, Canada, and the United States, just to name a few partners in the effort. The International Space Station (ISS) offers this multinational platform for a manned presence in orbit, which requires logistic support to resupply astronauts from multiple sources. One source of resupply comes from the Japan Aerospace Exploration Agency's (JAXA) H-II Transfer Vehicle (HTV), known as Kounotori. The HTV spacecraft are unmanned cargo transport spacecraft used to deliver supplies to the ISS. Since the first test flight of the HTV spacecraft in 2009, regular flights to the ISS have been conducted. The third HTV flight, known as HTV-3, flew to the ISS from July 21, 2012 (launch) to July 27, 2012 (capture by ISS). HTV-3 successfully re-entered the atmosphere on September 14, 2012, completing the resupply mission to the space station. In Figure 1.1 the main engines of the HTV spacecraft are clearly visible on an HTV spacecraft in orbit. A close view of the main orbital propulsion engine and nozzle contour is provided in Figure 1.2 


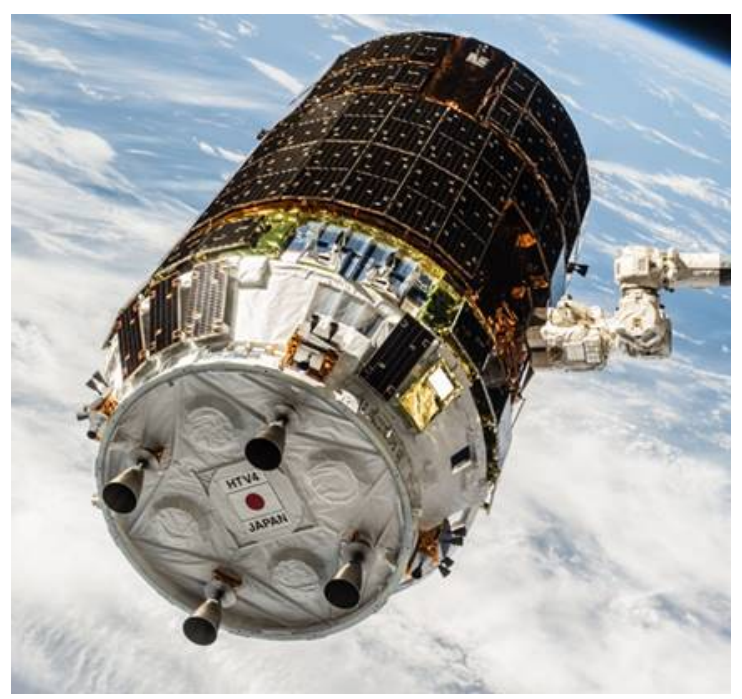

Figure 1.1: HTV 2 Spacecraft.

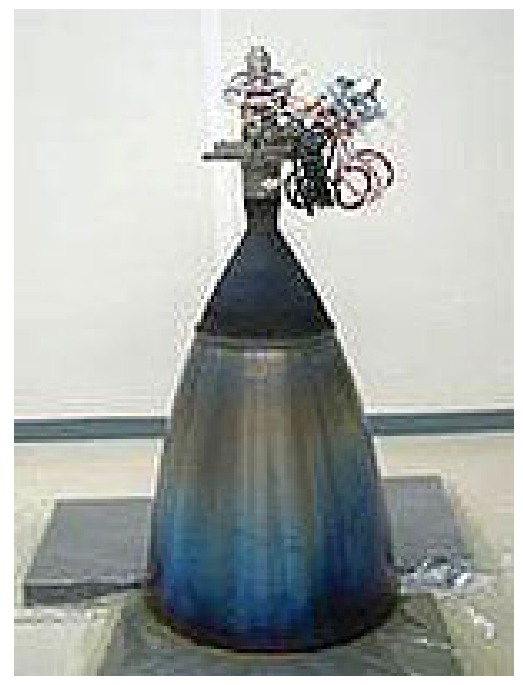

Figure 1.2: HTV Mach 5 Main Engine.

During approach maneuvers with the International Space Station, as seen in Figure 1.3 , the HTV-3 spacecraft performed an emergency procedure during the approach phase of the mission flight profile ${ }^{1}$ in order to decrease the closing rate as the spacecraft approached the space station. The procedure for decreasing the closing rate was to activate the four orbital propulsion jets on the spacecraft, and keep them activated for a set period of time. 


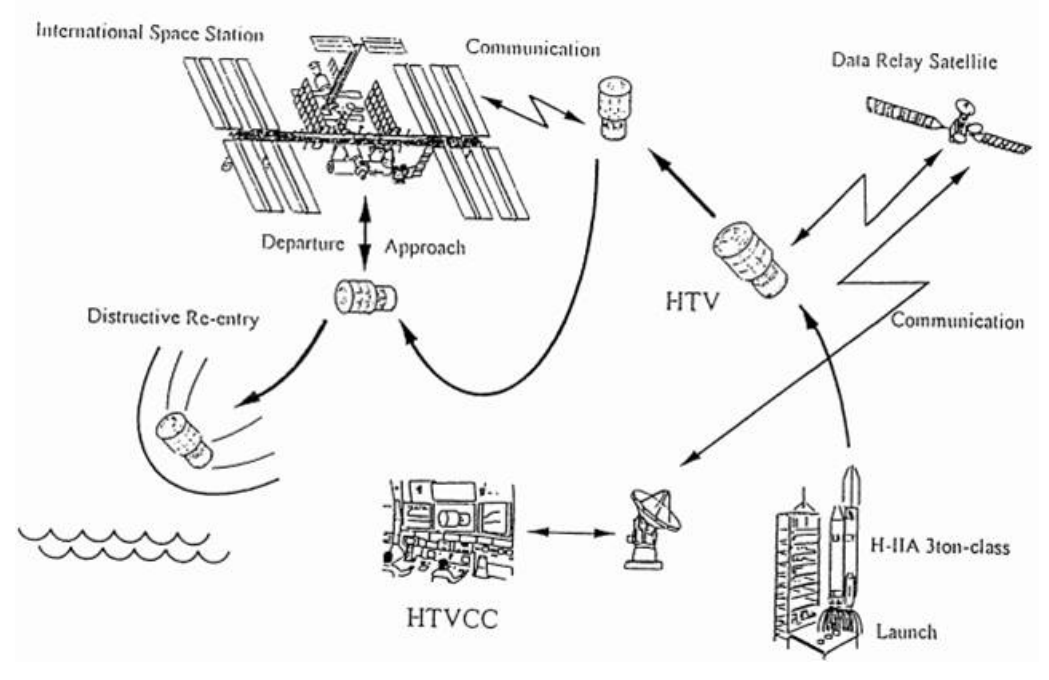

Figure 1.3: HTV ISS Mission Profile.

NASA Johnson Space Center had performed Direct Simulation Monte Carlo, referred to as DSMC, fluid dynamics simulations in order to provide the approved maneuver procedures for all spacecraft operating in the vicinity of the ISS. The analysis was conducted, using the NASA DSMC Analysis Code (DAC), of the flow field resulting from the propulsion thrusters on the HTV series spacecraft. The prediction showed minimal jet-jet interaction, as well as certifying that the emergency deceleration maneuver was safe within the proximity of the space station. When the HTV-3 spacecraft performed the maneuver, however, it resulted in exterior damage to solar cells and the main body of the International Space Station. With the occurrence of damage from the maneuver, NASA Johnson Space Center determined that their DAC prediction did not satisfactorily model the complex fluid interaction occurring in the exhaust plumes from the HTV orbital propulsion jets. Figure 1.4 below shows output of the DAC simulation that NASA Johnson Space Center conducted of the HTV / ISS interaction following the incident. ${ }^{2}$ Within Figure 1.4 the predicted number density, $N d$, and the total energy flux, $Q_{t o t a l}$, for the simulated flowfield are shown. 


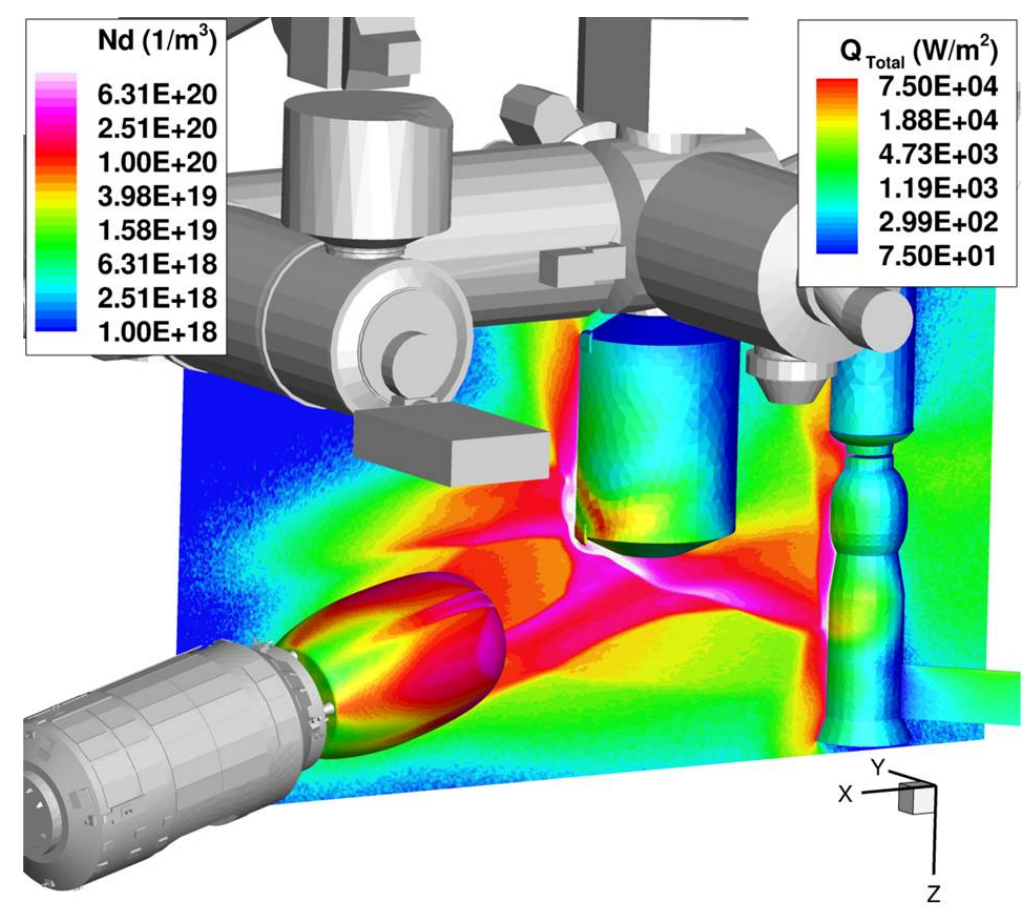

Figure 1.4: NASA DSMC Simulation of Event.

NASA Johnson Space Center subsequently awarded a research grant to the University of Virginia Aerospace Research Laboratory, specifically the Flowfield Diagnostics Laboratory, to conduct experimental research in order to provide data to refine the DAC predictive model. The additional refinement of the analysis algorithm will directly result in changes to spaceflight procedures surrounding the approach and docking / capture process with the International Space Station.

\section{$1.1 \quad$ Research Objectives}

The over-arching objective for this research is to investigate a hypersonic rarefied gas flowfield that is representative of the fluid flow encountered during the incident between the HTV and ISS. In order to achieve this, there are two primary investigation objectives to this research. The first objective is to study the interaction of parallel Mach 5 under-expanded jets. This will involve experiments where Mach 5 jets are allowed to exhaust into a quiescent atmosphere. The goal of these experiments will 
be to determine the effect of center-to-center spacing and number of exhausting jets (two jet versus four jet configurations) on jet-jet interactions, as well as quantify the mixing resulting from the jet-jet interactions.

The second research objective is to determine the impact of the introduction of an impingement surface on the jet-jet interactions. By introducing an impingement surface into the flowfield at a number of standoff distances, this investigation will offer insight into the effect of the impingement surface on flowfield geometry. The introduction of an impingement surface into the flowfield will also allow for the characterization of the impact of the impingement surface on neighboring jet interaction and mixing.

In order to achieve these two main objectives, two experimental approaches will be taken. First, broadband Planar Laser Induced Iodine Fluorescence (PLIIF) will be used for flowfield visualization. This experimental approach will provide the data necessary to determine the effect of center-to-center spacing on jet-jet and jet-jetimpingement surface interactions. Secondly, PLIIF quantitative density ratio imaging will be employed to quantify the mixing resulting from the jet-jet and jet-jetimpingement surface interactions. By focusing on these two research objectives, a thorough investigation into the jet-jet interactions, and the contribution to mixing from the introduction of an impingement surface, can be completed. Thorough insight into the fluid dynamics involved in the flight incident will lead to significant improvements in computational modeling of these complex fluid flows. 


\section{Chapter 2}

\section{BACKGROUND}

High speed fluid flows, especially under-expanded jet plumes, have been of particular interest to the aerospace industry since the beginning of high performance flight. A common example of the under-expanded jet plume is the exhaust of a rocket at high altitude. Previous research efforts in this field have focused on characterizing and collecting empirical data for under-expanded jets, in a variety of experimental conditions. Research has also been conducted into the numerical modeling and simulation of hypersonic flow fields in a variety of conditions.

A basic overview of compressible nozzle flow principles will provide a foundation for the discussion of under-expanded jets contained in the following chapters. Flow through a nozzle contour results from gas flowing from a high pressure region, called a plenum, to a low back pressure region. The high pressure plenum is of sufficient size such that any flow velocities are considered negligible. The nozzle contour utilized in this research is a converging-diverging nozzle, which consists of a section of decreasing cross-sectional area, a throat at the smallest cross-section, followed by a section of increasing cross-sectional area. Due to the pressure differential across the nozzle contour, the gas flows from the high pressure plenum into the converging portion of the nozzle, through the throat and then through the diverging portion of the nozzle contour. The gas then exhausts into the background region as a jet plume.

In a steady quasi-one dimensional internal, isentropic, inviscid flow the Mach

number can reach a maximum of one at a minimum in the cross-sectional area of 
the nozzle, and the nozzle is referred to as 'choked'. When the flow is subsonic in the diverging nozzle, the nozzle is not considered to be 'choked'. As the pressure differential increases, the flow speed increases in the nozzle. With continual increase in the pressure differential, the flow speed at the throat cross-section will reach Mach one. With continued increase in the pressure differential, the flow in the diverging contour will become supersonic. The shape of the jet plume and the flow pattern downstream of the nozzle exit will change based on the back pressure, but the velocity within the nozzle contour will remain constant at the choked flow conditions. The nozzle mass flow cannot be increased with a further decrease in back pressure, though it can be increased by an increase in plenum pressure.

Since the research described herein is concerned with under-expanded jet plume interactions, only the under-expanded case will be covered in this overview. Figure 2.1 shows a diagram of an under-expanded jet plume.

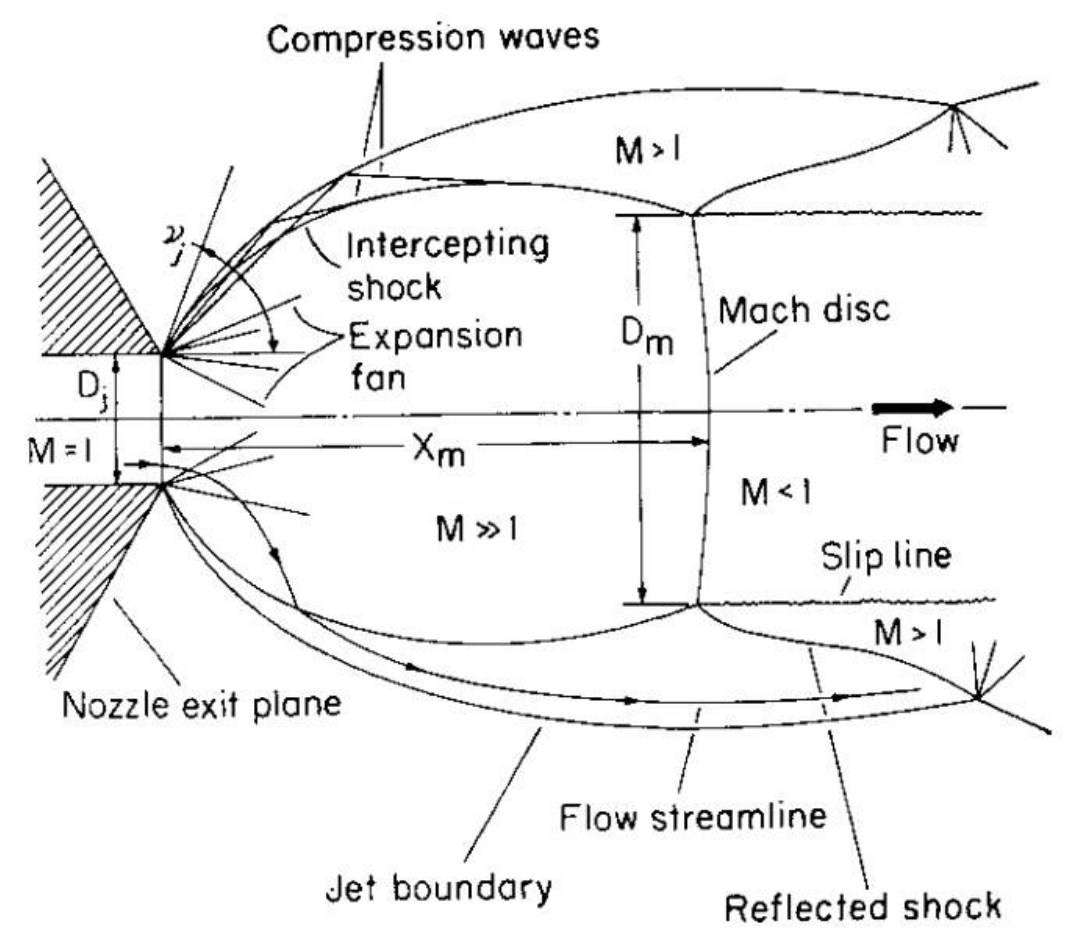

Figure 2.1: Diagram of an under-expanded jet structure.

Terming the flow under-expanded simply means that the flow must undergo an 
expansion to match the difference between the higher nozzle exit pressure and the lower ambient back pressure. In order to facilitate this expansion, a series of expansion waves, known as a Prandtl-Meyer expansion fan, forms at the nozzle exit lip. These waves cause the flow to turn outward and reduce in pressure. These expansion waves extend until they reach the free pressure boundary. At the free pressure boundary, the expansion waves will be reflected as compression waves which will coalesce to form the intercepting shock at the edge of the jet core. This intercepting shock takes the form of an oblique shock, behind which the flow remains supersonic but at a reduced Mach number compared to the flow in the jet core. As the weak compression waves converge downstream, if the compression is large enough, the compression waves will combine to form a normal shock perpendicular to the centerline flow direction. In the under-expanded jet case, the pressure in the jet core expands to less than the back pressure and is increased across the normal shock. Across the normal shock the pressure of the gas is increased, but may result in a pressure that is now greater than that of the back pressure. Because the pressure is now greater than the back pressure, the flow again turns outward and creates another series of expansion and subsequent compression waves downstream of the normal shock.

Substantial experimental research has been conducted in order to understand

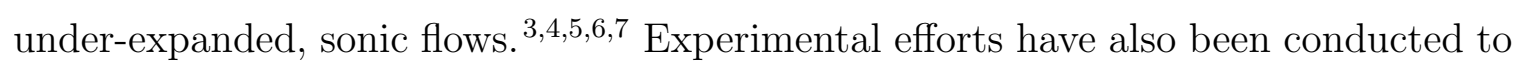
determine the Mach disk location and general shock geometry of under-expanded jets with a variety of experimental conditions, including a wide range of pressure ratios, nozzle contours, and working fluids. $\frac{8|9| 10}{}$ Additionally, significant research efforts have focused on investigating boundary layer and shock interactions. $\frac{11|12| 13}{12}$ The interaction of closely spaced, parallel sonic jets has been investigated using Schlieren imaging and laser Doppler velocimetry. $\frac{14}{}$ Experimental research has also been conducted into under-expanded jets and shock structure using Rayleigh Scattering. ${ }^{[15}$ Investigations have been conducted on the interaction of jet plumes with impingement surfaces of 
various configurations. $\frac{16|17| 18}{18}$ While most of the previously listed research focused on continuum flow of under-expanded jet plumes, another significant aspect of these flow fields is under-expanded jet plumes that exhaust into extremely low back pressures, causing the fluid flow to transition from continuum flow to rarefied gas flow. In much the same way that Reynold's number explains certain flowfield characteristics, the Knudsen number is used to determine whether continuum mechanics or statistical mechanics should be used to accurately model a given flowfield. The Knudsen number is defined as:

$$
K n=\frac{\lambda}{L}
$$

where $\lambda$ is the mean free path and $L$ is a representative physical length scale. The mean free path is defined as the average distance traveled by a molecule, in this research diatomic nitrogen or iodine, between collisions. An overview of mean free path distances for various pressure ranges can be seen in Table 2.1, compiled from multiple sources $\frac{19 \mid 20}{}$. These values are generalizations and used solely for illustrative purposes. The representative physical length scale is typically based on a physical dimension of the system being studied. For example, the single jet flowfield detailed in Chapter 5 has a maximum predicted Knudsen number, just before the Mach disk, of 4820. It is generally accepted that a flow defined by a Knudsen number greater than one is rarefied, though one source specified a Knudsen number greater than ten to be the transition 21 . The table also shows the Knudsen number per millimeter, showing the transition from continuum to rarefied flow under medium vacuum conditions. 


\begin{tabular}{|c|c|c|c|}
\hline Pressure & Pressure & Mean Free Path & Kn $/ \mathbf{~ m m}$ \\
\hline Ambient Pressure & 760 torr & $68-80 \mathrm{~nm}$ & $6.8 \times 10^{-5}-8 \times 10^{-5}$ \\
\hline Low Vacuum & $225-0.75$ torr & $0.1-100 \mu \mathrm{m}$ & $1 \times 10^{-4}-0.1$ \\
\hline Medium Vacuum & 0.75 torr $-0.75 \mathrm{mTorr}$ & $0.1-100 \mathrm{~mm}$ & $0.1-100$ \\
\hline High Vacuum & 0.75 mTorr $-75 \mathrm{nTorr}$ & $10 \mathrm{~cm}-1 \mathrm{~km}$ & $100-1 \times 10^{6}$ \\
\hline
\end{tabular}

Table 2.1: Table of mean free path ranges and $\mathrm{Kn} / \mathrm{mm}$ for various atmospheric pressure/vacuum ranges.

For a Boltzmann gas, the mean free path can be calculated based on the Boltzmann constant and the temperature of the gas, therefore the Knudsen number can be re-written as:

$$
K n=\frac{k_{b} T}{\sqrt{(2) \pi d^{2} p L}}
$$

In equation 2.2, $k_{b}$ is the Boltzmann constant, $T$ is the thermodynamic temperature of the gas, $d$ is the hard shell diameter of the particle. This is determined based on the assumption that the particle can be represented as a 'hard' sphere of constant diameter. Finally, $p$ is the total pressure. The principles of rarefied gas dynamics are thoroughly explained in Kennard's and in Vincenti and Kruger's pioneering textbooks $22 \mid 23$.

Along with the collection of experimental data, a significant amount of research has been conducted into modeling and simulating these complex flow fields. As with most numerical simulations, experimental data is regularly used to validate the modeling techniques and methodology. A primary method for numerically analyzing supersonic flows, whether in a nozzle contour or exhausting into a background, is the method of characteristics applied to the compressible flow governing equations. Research by Owen and Thornhill ${ }^{24}$ and Love et. al. ${ }^{25}$ outlined the process of creating characteristic equations and compatibility equations to numerically analyze a supersonic flowfield.

Based on the overview in Anderson's compressible flow textbook ${ }^{26}$, there are three 
primary steps in applying the method of characteristics to compressible flow:

1. For two-dimensional compressible flow, it is necessary to find particular lines in the $\mathrm{x}-\mathrm{y}$ space where the primary flow variables (pressure, density, temperature, and velocity) are continuous, but along which the derivatives of the primary flow variables are indeterminate. These unique lines are known as characteristic lines.

2. Compatibility equations are necessary to solve for the primary flow variables along the characteristic lines. By combining the partial differential conservation equations, ordinary differential equations are obtained. These ordinary differential equations will hold only along a characteristic line.

3. The compatibility equations are solved along the characteristic lines in a stepby-step fashion, starting with the known initial conditions at some point or region in the flowfield. In general, the characteristic lines depend on the flowfield and the compatibility equations are a function of geometric physical location along the characteristic lines, which means that the characteristic and compatibility equations must be solved simultaneously.

As a general introduction to the method of characteristics, the application of the previous three points will be covered further in the following section. For twodimensional steady, isentropic, irrotational supersonic flow, the governing non-linear equations are:

$$
\left(1-\frac{\Phi_{x}^{2}}{a^{2}}\right) \Phi_{x x}+\left(1-\frac{\Phi_{y}^{2}}{a^{2}}\right) \Phi_{y y}-\frac{2 \Phi_{x} \Phi_{y}}{a^{2}} \Phi_{x y}=0
$$

where $a$ is the speed of sound as a function of the velocity potential $\Phi$, and is defined as:

$$
a^{2}=a_{o}^{2}-\frac{\gamma-1}{2}\left(\Phi_{x}^{2}+\Phi_{y}^{2}+\Phi_{z}^{2}\right)
$$


which arises from $V=\nabla \Phi$ for irrotational flow. In Cartesian coordinates:

$$
\nabla \Phi=\frac{\partial \Phi}{\partial x} \hat{i}+\frac{\partial \Phi}{\partial y} \hat{j}+\frac{\partial \Phi}{\partial z} \hat{k}
$$

which simplifies to

$$
\nabla \Phi=\frac{\partial \Phi}{\partial x} \hat{i}+\frac{\partial \Phi}{\partial y} \hat{j}
$$

for two-dimensional flow. The components for the velocity vector can further be defined as:

$$
\begin{aligned}
& \frac{\partial \Phi}{\partial x}=\Phi_{x}=u \\
& \frac{\partial \Phi}{\partial y}=\Phi_{y}=v
\end{aligned}
$$

In order to solve for the final variables in equation $2.3, \Phi_{x}=f(x, y)$ and $\Phi_{y}=f(x, y)$ are assumed, resulting in the following relationship:

$$
\begin{aligned}
& d \Phi_{x}=\frac{\partial \Phi_{x}}{\partial x} d x+\frac{\partial \Phi_{x}}{\partial y}=\Phi_{x x} d x+\Phi_{x y} d y \\
& d \Phi_{y}=\frac{\partial \Phi_{y}}{\partial x} d x+\frac{\partial \Phi_{y}}{\partial y}=\Phi_{x y} d x+\Phi_{y y} d y
\end{aligned}
$$

Rewriting the above equations:

$$
\begin{aligned}
& \Phi_{x x} d x+\Phi_{x y} d y=d u \\
& \Phi_{x y} d x+\Phi_{y y} d y=d v
\end{aligned}
$$

Equations 2.3 and 2.9 can be treated as a system of linear, simultaneous, algebraic 
equations for compressible flow. Using Cramer's rule, the solution for $\Phi_{x y}$ becomes:

$$
\Phi_{x y}=\frac{\left|\begin{array}{ccc}
1-\frac{u^{2}}{a^{2}} & 0 & 1-\frac{v^{2}}{a^{2}} \\
d x & d u & 0 \\
0 & d v & d y
\end{array}\right|}{\left|\begin{array}{ccc}
1-\frac{u^{2}}{a^{2}} & -\frac{2 u v}{a^{2}} & 1-\frac{v^{2}}{a^{2}} \\
d x & d y & 0 \\
0 & d x & d y
\end{array}\right|}=\frac{N}{D}
$$

Equation 2.10 is generally held to represent a combination of continuity, momentum, and energy equations for the two-dimensional, steady, isentropic, irrotational, compressible flow. If $d x$ and $d y$ are chosen such that the denominator $D$ is equal to zero, $N$ must equal zero as well. This results from maintaining that $\Phi_{x y}$ must be defined at a given point in the flow, which means keeping $\Phi_{x y}$ finite. By enforcing this condition, $\Phi_{x y}$ is indeterminate for this set of $d x$ and $d y$, and equations for the characteristic lines can be determined. When $D=0$, the denominator of equation 2.10 yields:

$$
\left(1-\frac{u^{2}}{a^{2}}\right) d y^{2}+\frac{2 u v}{a^{2}} d x d y+\left(1-\frac{v^{2}}{a^{2}}\right) d x^{2}=0
$$

Re-arranging the previous equation by dividing by $d x^{2}$ :

$$
\left.\left(1-\frac{u^{2}}{a^{2}}\right) \frac{d y^{2}}{d x^{2}}\right|_{\text {char }}+\left.\frac{2 u v}{a^{2}} \frac{d y}{d x}\right|_{\text {char }}+\left(1-\frac{v^{2}}{a^{2}}\right)=0
$$

Applying the quadratic equation to solve for $\left.\frac{d y}{d x}\right|_{c h a r}$ yields:

$$
\left.\frac{d y}{d x}\right|_{\text {char }}=\frac{-\frac{u v}{a^{2}} \pm \sqrt{\frac{u^{2}+v^{2}}{a^{2}}-1}}{1-\frac{u^{2}}{a^{2}}}
$$


The above equation defines the characteristic line in the physical Cartersian space. The term under the radical in equation 2.13 allows for the classification of the characteristic solutions. Examining the term under the radical, and re-arranging using the components of the velocity vector and the definition of the Mach number:

$$
\frac{u^{2}+v^{2}}{a^{2}}-1=\frac{V^{2}}{a^{2}}-1=M^{2}-1
$$

The three possible solution ranges for $M$ yield:

1. If $M>1$, there are two real characteristics through each point in the flowfield. This means that equation 2.3 is defined by a hyperbolic partial differential equation

2. If $M=1$, there is one real characteristic through each point in the flowfield. This means that equation 2.3 is a parabolic partial differential equation.

3. If $M<1$, the characteristics are imaginary and equation 2.3 is an elliptic partial differential equation.

The flowfields investigated in this research are governed by Mach numbers greater than one. Returning to equation 2.13 and using the definition of velocity components at any point in the flowfield, $u=V \cos (\theta)$ and $v=V \sin (\theta)$, as well as defining the Mach angle $\mu$ as $\mu=\arcsin \left(\frac{1}{M}\right)$, equation 2.13 reduces to:

$$
\left.\frac{d y}{d x}\right|_{\text {char }}=\tan (\theta \mp \mu)
$$

With the final form of the characteristic equation shown above, it is now necessary to address the derivation of the compatibility equation.

To determine the compatibility equations, the numerator of equation 2.10 is set 
to zero. This yields:

$$
\begin{gathered}
\left(1-\frac{u^{2}}{a^{2}}\right) d u d y+\left(1-\frac{v^{2}}{a^{2}}\right) d x d v=0 \\
\frac{d v}{d u}=-\frac{\left(1-\frac{u^{2}}{a^{2}}\right)}{\left(1-\frac{v^{2}}{a^{2}}\right)} \frac{d y}{d x}
\end{gathered}
$$

Since $N$ only equals zero when $D$ equals zero, these will only hold for characteristic lines. This means $\frac{d y}{d x}=\left.\frac{d y}{d x}\right|_{\text {char }}$, and by substituting equation 2.13 into equation 2.17 (and using $u=V \cos (\theta)$ and $v=V \sin (\theta))$ yields:

$$
\frac{d(V \sin (\theta))}{d(V \cos (\theta))}=\frac{M^{2} \cos (\theta) \sin (\theta) \mp \sqrt{M^{2}-1}}{1-M^{2} \sin ^{2}(\theta)}
$$

Which in turn reduces to:

$$
d \theta=\mp \sqrt{M^{2}-1} \frac{d V}{V}
$$

This is known as the compatibility equation which describes the variation of the flow properties along a characteristic line. By integrating this equation, the PrandtlMeyer function $v(M)$ becomes a part of the compatibility equation. From this integration, the above equation can be re-written as the algebraic compatibility equations:

$$
\begin{aligned}
& \theta+v(M)=\text { constant }=K^{-}\left(\text {along the } C^{-} \text {characteristic }\right) \\
& \theta-v(M)=\text { constant }=K^{+}\left(\text {along the } C^{+} \text {characteristic }\right)
\end{aligned}
$$

These equations relate the velocity magnitude and direction along the characteristic lines. These equations are independent of spatial location, which is not true for the more complex solutions such as those for axisymmetric irrotational flow. This methodology was employed early in the computational investigations of supersonic flowfields, and is still used to design supersonic nozzle contours. 
In order to determine the equations for the method of characteristics for axisymmetric irrotational flow, a cylindrical coordinate system must be used in place of the Cartesian system shown above. Using a cylindrical coordinate system, as shown in Figure $2.2 \sqrt{26}$, results in the use of $r, \phi$, and $x$, with corresponding velocity components $v, w$, and $u$ to determine the equations for the characteristic lines and the compatibility equation.

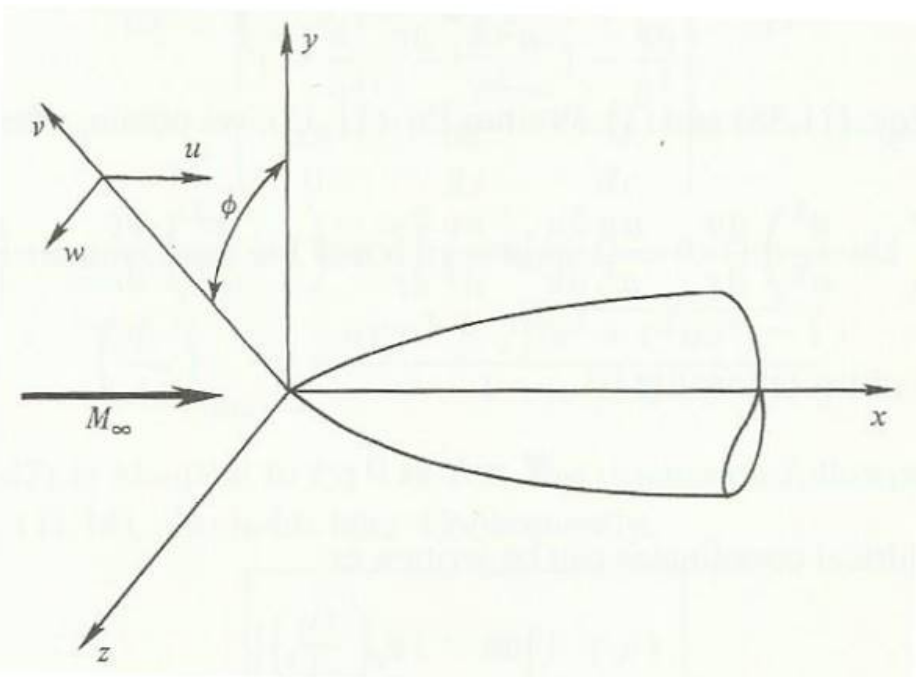

Figure 2.2: Cartesian coordinate system overlaid with cylindrical system.

Following Anderson's derivation in the Modern Compressible Flow textbook ${ }^{26}$, the characteristic lines in the cylindrical coordinate system can be written as:

$$
\left.\frac{d r}{d x}\right|_{\text {char }}=\tan (\theta \mp \mu)
$$

and the compatibility equation can be written as:

$$
\begin{aligned}
d(\theta+v) & =\frac{1}{\sqrt{M^{2}-1}-\cot (\theta)} \frac{d r}{r} \text { (along the } C^{-} \text {characteristic) } \\
d(\theta-v) & =-\frac{1}{\sqrt{M^{2}-1}+\cot (\theta)} \frac{d r}{r} \text { (along the } C^{+} \text {characteristic) }
\end{aligned}
$$


Comparing the equations for the characteristic lines between the two-dimensional irrotational flow and axisymmetric irrotational flow cases, it is shown that the change in coordinate system yielded the same definition for characteristic lines. On the other hand, when the compatibility equations for the axisymmetric irrotational flow, equation 2.22, are compared to the two-dimensional irrotational equations, equation 2.20, two major differences are apparent. First, the axisymmetric irrotational flow compatibility equation is no longer a simple algebraic equation, it has become a differential equation in $r$. Secondly, the compatibility equations are no longer constant along a characteristic line, instead they vary with respect to the spatial location in the flowfield, as dictated by the $\frac{d r}{r}$ term. The axisymmetric irrotational flow formulation was used in this research to determine the Mach 5 nozzle contour employed in the test sections, and was used as a secondary verification of desired neighboring jet interaction when determining the geometric design of the experiment. Both applications are outlined in Chapter 4 .

Further research into more accurately numerically modelling complex flowfields, such as under-expanded jets, has been reported. Godunov's paper ${ }^{27}$ represented the first major step in properly treating artificial viscosity to stabilize numerical oscillations in strong gradient regions, which led to a much better numerical prediction of shock regions. Many early methods were investigated in order to approximate the jet structure for under-expanded jet plumes. $\frac{28|29| 30}{20}$ As computing power increased, and became readily available, computational techniques for modeling complex high speed flow fields also evolved. Popular methods for numerical solutions to the Navier-Stokes equations $^{31 / 32[33}$ include finite difference schemes ${ }^{34}$, finite volume methods ${ }^{\sqrt{35 \mid 36}}$, and the Reynolds Averaged Navier-Stokes (RANS) methodology. ${ }^{37}$ While some or most of these continuum techniques have been incorporated into commercial simulation software, modeling of rarefied flow fields has also seen significant development. Bird outlined the statistical DSMC method ${ }^{[38}$, and Boyd ${ }^{39}$ outlined a computationally more 
efficient method of simulating rarefied flows.

\subsection{New Research in Context}

The experimental effort outlined in this dissertation differs from those previously mentioned in that it focuses on injecting room temperature nitrogen, seeded with iodine, through a set of four parallel Mach 5 exit velocity nozzles. The objective is to image and collect qualitative and quantitative data for an analogue of the Japanese HTV series of autonomous spacecraft. This investigation is novel both in the hypersonic flow being studied, as well as the application of the specific imaging technique utilized by the Flow Diagnostics Laboratory at the University of Virginia. Using Planar Laser Induced Iodine Florescence (PLIIF) ${ }^{40 \mid 41}$, the lab is able to collect both qualitative and quantitative data on the flow field of interest. No efforts using PLIIF to image a hypersonic flowfield of this complexity have been attempted. As part of this research effort, studies have been made to quantify the interaction effects of these hypersonic parallel under-expanded closely-spaced jets, as well as the interaction of the jets with an impingement surface. The flat surface will serve as an analogue for the interaction that occurred between the HTV spacecraft and the International Space Station. While research into flat plate interactions with jet plumes has been conducted in the past ${ }^{42 / 43 / 44}$, research into hypersonic jet-jet and jet-jet-surface interactions provides a new and novel investigation. This research is applicable to realworld spaceflight conditions and operational procedures. The data collected from this research will directly influence recommendations and procedures that NASA Johnson Space Center provides to all entities (governmental and commercial) involved in spaceflight to the International Space Station. 


\section{CHAPTER 3}

\section{LASER-INDUCED FluOREsCEnCE TheORY}

The research presented herein, investigating hypersonic interacting flow fields, takes advantage of the fluorescence properties of the iodine molecule when excited by radiative energy in the form of laser photon emission. ${ }^{45}$ The technique, known as Planar Laser-Induced Iodine Fluorescence (PLIIF), is a non-intrusive optical technique that captures time-averaged, spatially-resolved measurements in the subject flow field. This measurement technique has been used extensively at the University of Virginia

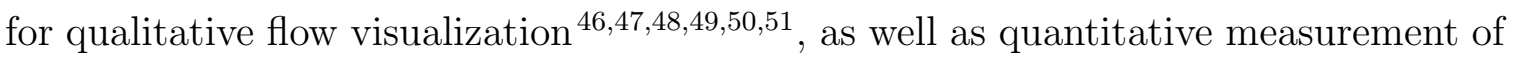
species concentration, $48 / 49150 \mid 51$ density and velocity $466147|48| 49150 \mid 51$.

While there are numerous methods that have been applied to qualitative and quantitative data collection on high speed flows, there are shortcomings in each of the major techniques that make the PLIIF method the optimal technique with which to study this unique flow field. The PLIIF technique is advantageous over other line-of-sight techniques, such as Schlieren imaging, because in line-of-sight techniques the signal is integrated over the beam path length, which results in a lack of spatial resolution within the target flow field. Schlieren also cannot image the rarefied flow regime because of the minute index of refraction changes that occur in the very low flow field density. A fluorescence technique that has been applied to rarefied flow fields, known as electron beam fluorescence, will provide qualitative data on the flow field. The shortfall of the electron beam fluorescence technique is that it lacks the ability to provide the necessary quantitative thermodynamic and velocity 
measurements for a mixed flow field. ${ }^{52 / 53}$ In this instance, a mixed flow field refers to the flow field of interest containing both continuum and rarefied flow regimes, which is exactly the type of flow conditions found in this research. Finally, the other major imaging technique known as Particle Image Velocimetry (PIV) is limited in its application in rarefied flow conditions by the velocity slip between the seed particles and the carrier molecules that occurs under rarefied conditions. In contrast to PIV, the PLIIF technique can produce pixel-accurate quantitative velocity measurements in rarefied flow, and in mixed flow field conditions.

\subsection{Iodine Model and Governing Rate Equation}

\subsubsection{Molecular Iodine Energy Transitions}

In order to fully understand the PLIIF technique, a look at the interaction of the diatomic iodine molecule with laser excitation is necessary. Energy is absorbed by a diatomic iodine molecule when the laser radiation is resonant with the energy separation between two unique energy levels in the molecule. When energy is absorbed, the molecule will undergo a transition from a lower energy level to an excited upper energy level, as shown in Figure $3.1^{54}$. 


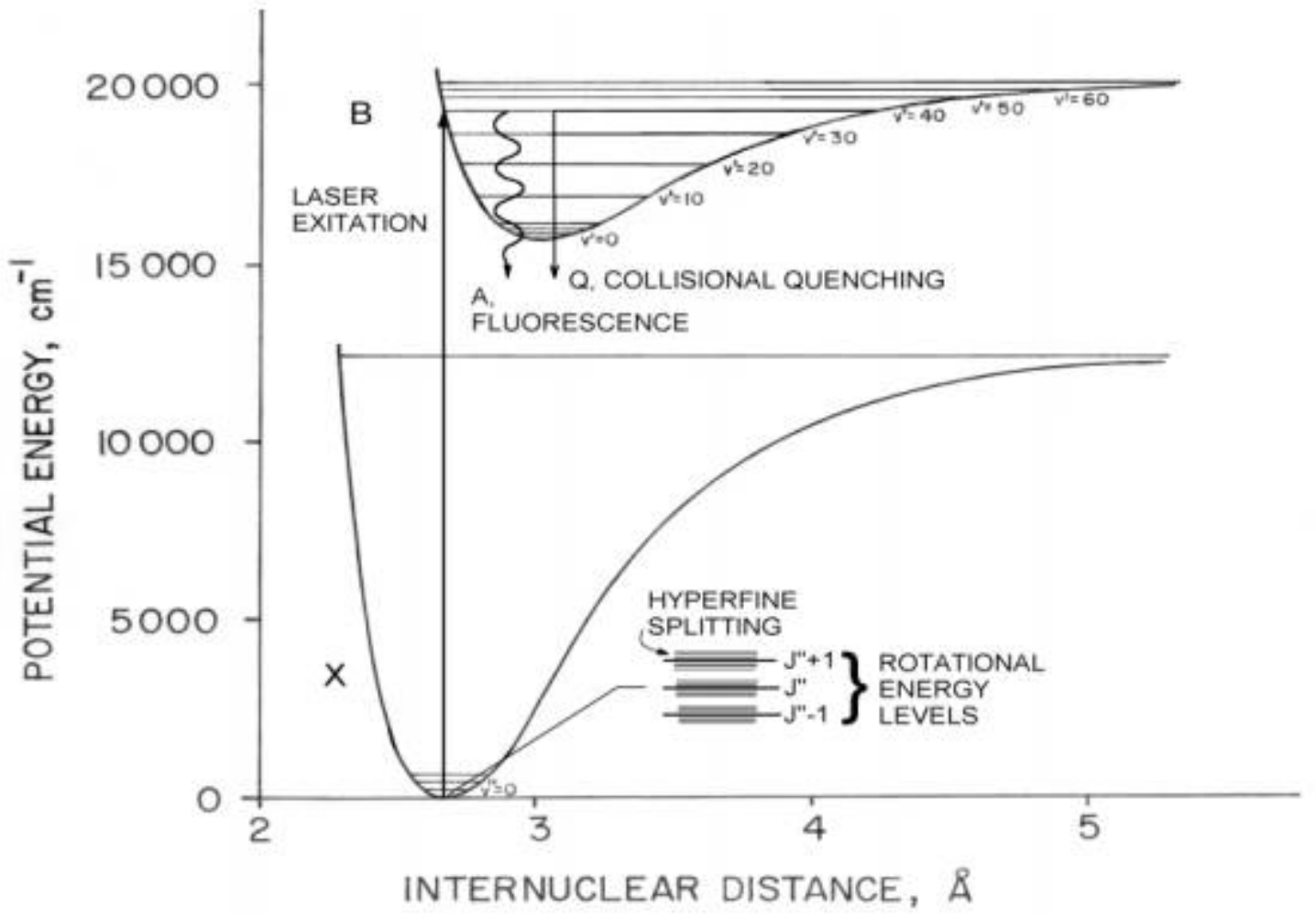

Figure 3.1: Energy Level Diagram for the Diatomic Iodine Molecule.

Shown in Figure 3.1 are the vibrational, rotational, and hyperfine splitting of the energy levels. Diatomic iodine molecules in the electronic ground state, denoted by $\mathrm{X}$, are resonant with energy levels in the excited B-state when using the 514.5 nanometer wavelength radiation emitted from an argon-ion laser. When the molecule is subjected to radiation in this wavelength range, the molecule will become excited to an upper electronic B-state. The transition from the ground energy state to the upper excited energy state is shown in Figure 3.2 . 


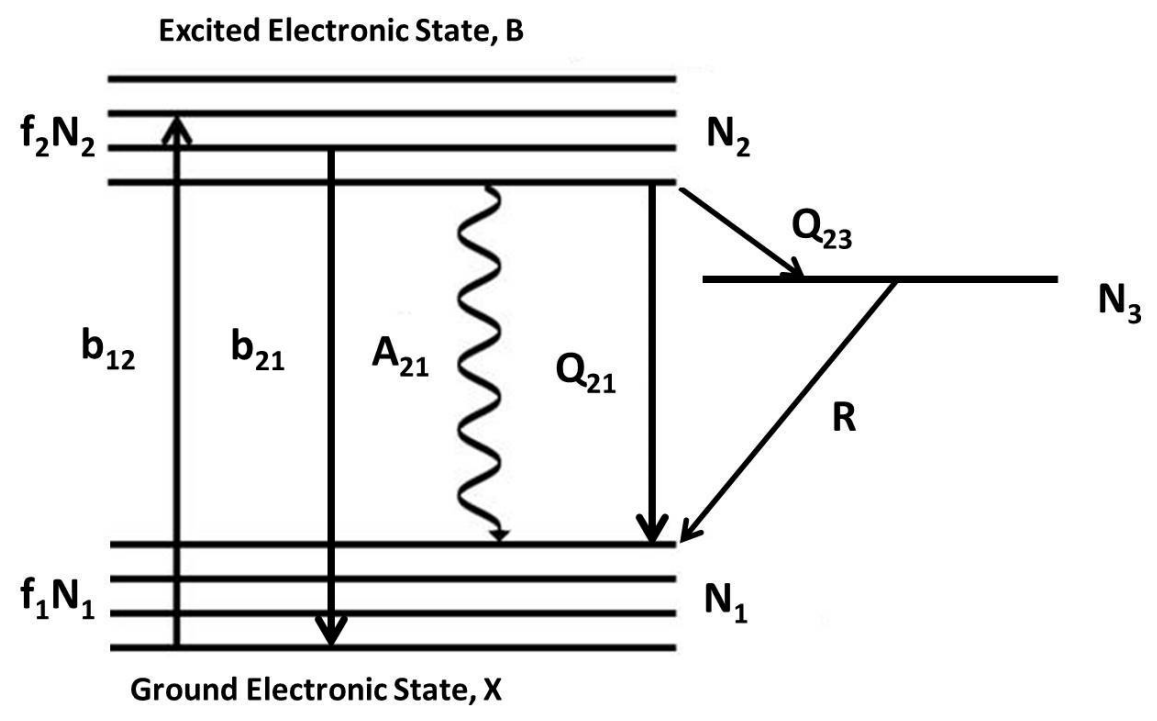

Figure 3.2: Diagram of Energy Transfer Mechanisms for Diatomic Iodine.

In order to take advantage of the ability to excite iodine atoms into the upper Bstate, an understanding of energy transfer mechanisms for the return to the ground X-state is necessary. In Figure $3.2, N_{1}$ denotes the total population of available iodine molecules in the ground electronic X-state, where $N_{2}$ represents the total population of available iodine molecules in the excited B-state. The associated fractions of molecules in the rotational or vibrational quantum configurations corresponding to states resonant with the laser are denoted with the corresponding $f_{1}$, or $f_{2}$. The term $b_{12}$ refers to the rate at which diatomic iodine molecules become excited into the upper B-state. Once in the B-state, the molecule will redistribute to other rotational and vibrational quantum levels through molecular collisions and decay back into the ground X-state through four primary energy relaxation mechanisms. First, the molecule can return to the ground state through stimulated emission, denoted by $b_{21}$ in Figure 3.2 , which is when the molecule is stimulated back to the ground state by laser radiation. This is very small for the low laser energies of the current work. $A_{21}$, or spontaneous emission (fluorescence), is another method of energy relaxation, 
and the one of interest to the PLIIF technique. Collisional quenching, $Q_{21}$, is another energy transfer method which corresponds to inelastic collision with other molecules in the flow field. Lastly, pre-dissociation of the diatomic iodine molecule, referred to by $Q_{23}$, occurs if a third dissociative energy state overlaps the excited B-state the molecule resides in. It has been estimated in previous research that around one percent of the iodine molecules transition to the $N_{3}$ state ${ }^{40}$, therefore pre-dissociation is considered negligible in the application of this technique.

Another method of energy transfer is the process of molecular dissociation. This process can occur if the excitation source contains the photon energy required to separate the diatomic iodine molecule into individual iodine atoms. This method of energy transfer is not considered in this research because the photons from the 514.5 nanometer argon-ion laser used are not sufficiently energetic. On the other hand, dissociation does occur with diatomic iodine and an argon-ion laser operating in the 488 nanometer wavelength range.

\subsubsection{Governing Rate Equation}

To examine the details of the PLIIF imaging technique, determination of a governing rate equation for the excitation and relaxation of the diatomic iodine molecule is needed. Based on experimental conditions in this research, spontaneous emission (fluorescence) and collisional quenching are the only significant energy decay mechanisms to consider when formulating a governing rate equation. A thorough derivation of the rate equation model for iodine seeded into a reference gas is developed in Reference 40. With this in mind, a population decay rate equation can be written for the B-to-X state transition as follows:

$$
\frac{\partial N_{2}}{\partial t}=b_{12} f_{1} N_{1}-\left(A_{21}+Q_{21}\right) N_{2}
$$


In Equation 3.1, the first term on the right of the equal sign denotes the excitation of diatomic iodine from a single rotational/vibrational level in the X-state to the Bstate. The second term corresponds to the decay of molecules from the B-state to the X-state through spontaneous emission and collisional quenching. It should be noted here that this equation implicitly contains the assumption that $A_{21}$ and $Q_{21}$ are constant across all B-state energy levels. This is a valid assumption because the PLIIF technique collects the fluorescence signal from all B-state energy level wavelengths, therefore the specifics of which vibrational or rotational energy levels are populated in the excited B-state is inconsequential.

Since the argon-ion laser used in this research is a continuous wave laser, the change in excited iodine molecules with respect to time is equal to zero:

$$
\frac{\partial N_{2}}{\partial t}=0
$$

Using Equation 3.2 and Equation 3.1, it can be shown that, for a laser where the laser linewidth is less than the absorption linewidth of iodine, the solution to Equation 3.1 results in Equation 3.3 for the fluorescence signal resulting from the excitation of a single rotation-vibrational transition in the X-state to the B-state ${ }^{40}$ :

$$
S_{f}=C f_{v^{\prime \prime} J^{\prime \prime}}\left[\frac{A_{21}}{A_{21}+Q_{21}(p, T)}\right] \frac{V(p, T, u, v)}{\Delta v_{D}(T)} I f_{s} n
$$

The fluorescence intensity, $S_{f}$, is a function of several parameters. $C$ is a constant that is unique to this experimental setup and is dependent on the optical collection parameters. It also contains the Frank-Condon and Hönl-London spectroscopic parameters, which are constants, dependent on the iodine absorption transitions. The Boltzmann population fraction, $f_{v^{\prime \prime}} J^{\prime \prime}$, is a measure of the distribution of the rotational and vibrational energy level population in the ground state which is resonant with the argon-ion laser. This was denoted in Equation 3.1 as $f_{1}$. The Boltzmann 
population fraction is dependent on flow field temperature. The Stern-Volmer factor, shown above in brackets, is a measure of fluorescence efficiency. The fluorescence efficiency is measured as a ratio of the fluorescence, $A_{21}$, to the total available decay mechanisms, $A_{21}+Q_{21}$. The collisional quenching for excited molecules is directly related to temperature and pressure. $V(p, T, u, v)$ is the Voigt lineshape function and is a convolution of pressure and thermal broadening effects on the fluorescence spectrum. The Voigt lineshape is normalized by the Doppler broadening term, $\Delta v_{D}(T)$. The laser intensity, $I$, is a measure of the Gaussian distribution of laser power. The ratio of iodine to nitrogen in the flow field is accounted for by the seeding fraction, $f_{s}$, and is 1 part $I_{2}$ to 7000 parts $N_{2}$ for the experiments detailed in this work. The number density of the flow field is given by the term $n$ in Equation (3.3). It is seen that $S_{f}$ is not proportional to $n$ since the other parameters depend on pressure and temperature. Each of these terms will be covered in-depth in a subsequent subsection of this Chapter.

\subsubsection{Boltzmann Population Fraction}

The Boltzmann population fraction is a measure of the distribution of iodine molecules in the rotational and vibrational energy levels in the ground state, $\mathrm{X}$, excited by the laser. The population fraction is a combination of population contributions from unique rotational and vibrational quantum configurations in the X-state. Using $v^{\prime \prime}$ to denote vibrational states, and $J^{\prime \prime}$ to denote rotational states, the Boltzmann population fraction can be written as:

$$
f_{v^{\prime \prime} J^{\prime \prime}}(T)=f_{v^{\prime \prime}}(T) f_{J^{\prime \prime}}(T)
$$

The above formulation of the Boltzmann population assumes independent distributions of rotational and vibrational quantum states. Within Equation 3.4 , the 
contribution to total population fraction from vibrational quantum states can be written as:

$$
f_{v^{\prime \prime}}=\exp \left(-\nu^{\prime \prime} \frac{\theta_{v i b}}{T}\right)\left[1-\exp \left(-\frac{\theta_{v i b}}{T}\right)\right]
$$

In Equation 3.5, $\nu^{\prime \prime}$ denotes the specific vibrational quantum number of the level excited by the laser. The characteristic vibrational temperature for diatomic iodine is expressed as $\theta_{v i b}$, where:

$$
\theta_{v i b}=308.62 \mathrm{~K}
$$

The numerical value for the characteristic temperature of iodine is available from spectroscopic studies for diatomic molecules reported by Herzberg 55 . The vibrational contribution to the Boltzmann population can be seen by plotting the population fraction for various temperatures. Figure 3.3 shows the vibrational population fraction for a range of vibrational quantum numbers and temperatures. As the temperature increases, higher quantum vibrational states become populated. In the temperature ranges found in this research, which are 10 to $300 \mathrm{~K}$, the vibrational ground state, $v^{\prime \prime}=0$, contains the most significant portion of the population fraction. 


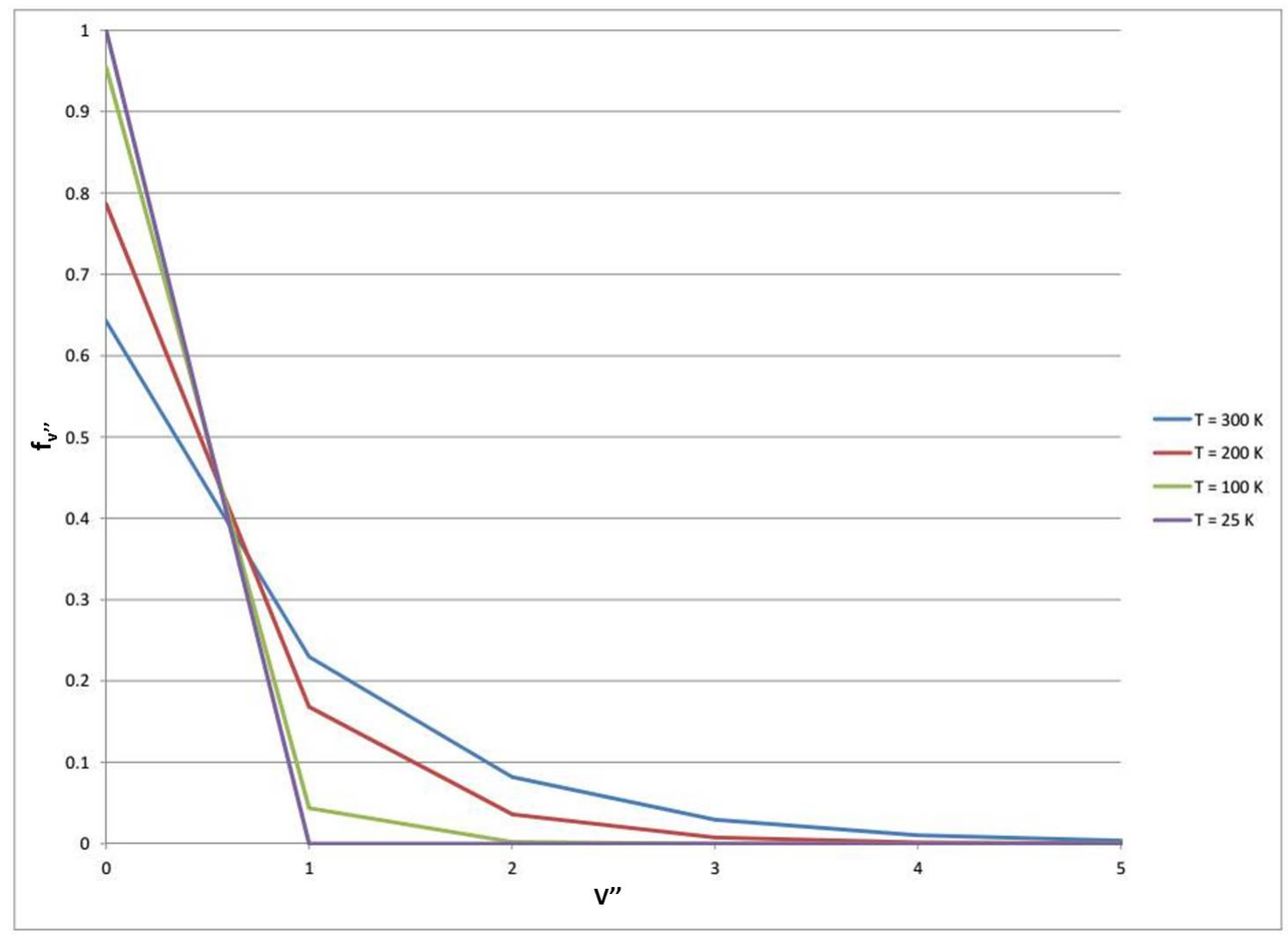

Figure 3.3: Boltzmann population fraction contribution from vibrational quantum states.

The rotational contribution to the Boltzmann population fraction can be written as:

$$
f_{J^{\prime \prime}}=\frac{\left(2 J^{\prime \prime}+1\right) \exp \left[-J^{\prime \prime}\left(J^{\prime \prime}+1\right) \frac{\theta r o t}{T}\right]}{\frac{T}{\sigma \theta_{r o t}}}
$$

where $J^{\prime \prime}$ is the rotational quantum number. The degeneracy of the rotational quantum state is $\sigma$, and is 2 for a symmetric diatomic molecule. The characteristic rotational temperature for diatomic iodine is given as ${ }^{55}$ :

$$
\theta_{\text {rot }}=0.0537 \mathrm{~K}
$$

By plotting the rotational population fraction, $f_{J^{\prime \prime}}$, versus rotational quantum numbers, $J^{\prime \prime}$, and for multiple temperatures, the contribution of a specific rotational 
level to the Boltzmann population fraction can be determined. This is shown in Figure 3.4. In Figure 3.4, the maximum population at room temperature occurs at a rotational quantum number of about 52. At the lowest temperature range encountered in this research, about $10 \mathrm{~K}$, the highest populated state occurs at a rotational quantum number of about 10 . The values of $J^{\prime \prime}$ excited by the argon laser at 514.5 nm are 13 and 15.

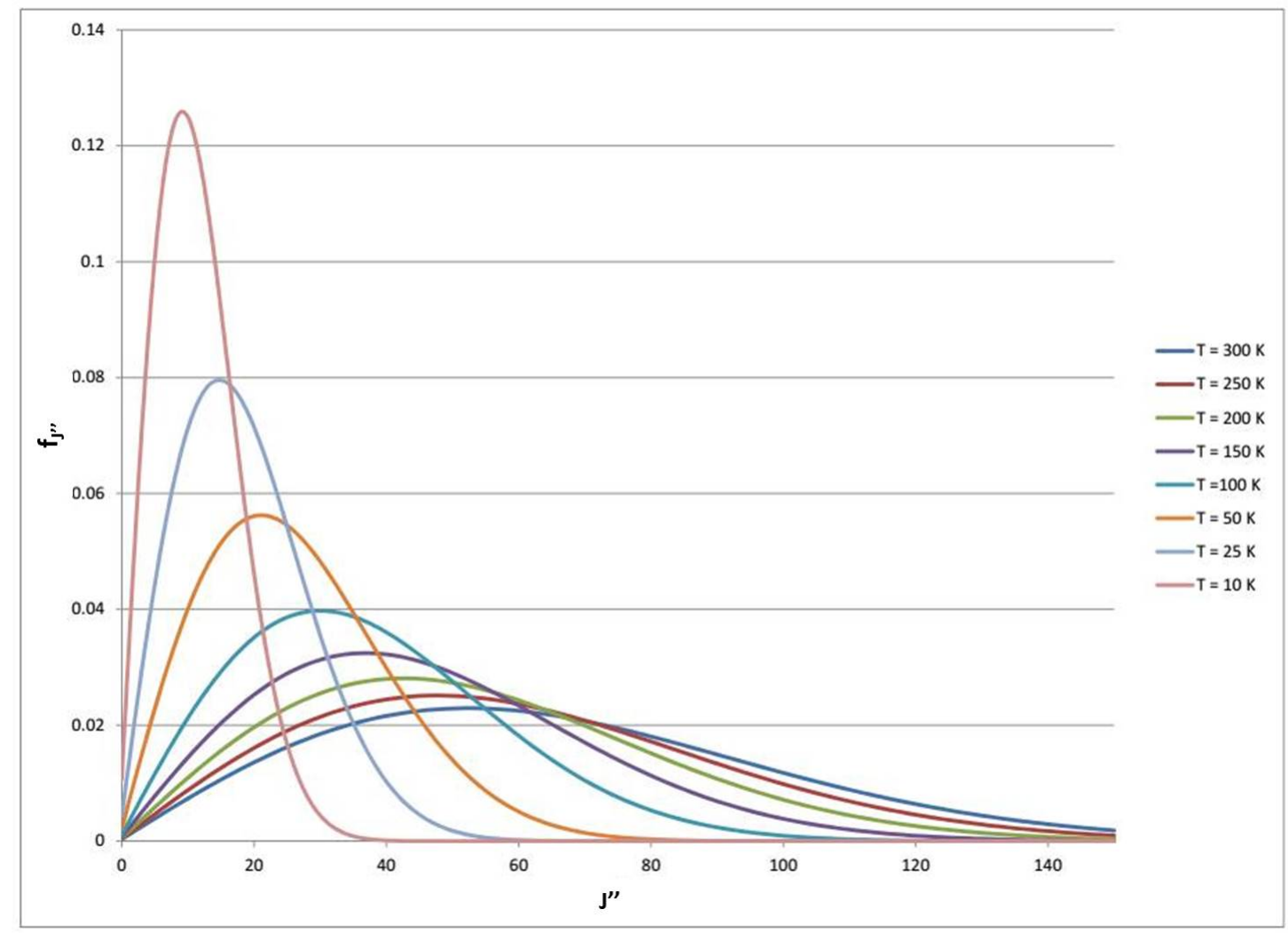

Figure 3.4: Boltzmann population fraction contribution from rotational quantum states.

The availability of access to the low rotational quantum number transitions of iodine, $J^{\prime \prime}=13$ and 15 , make the PLIIF technique utilized in this research a uniquely well-suited method for low temperature flow investigations. 


\subsubsection{Stern-Volmer Factor - Fluorescence Efficiency}

The Stern-Volmer factor is a measure of the fluorescence efficiency of excited diatomic iodine. Because the two primary methods of energy transfer are spontaneous emission, or fluorescence, and collisional quenching, the fluorescence efficiency can be written as:

$$
\frac{A_{21}}{A_{21}+Q(p, T)}
$$

In Equation 3.9, the term $A_{21}$ is the spontaneous emission, or fluorescence, and the $Q_{21}(p, T)$ is the pressure and temperature dependent collisional quenching of the iodine molecule. Re-writing Equation 3.9 in the form:

$$
\frac{A_{21}}{A_{21}+Q_{21}(p, T)}=\frac{1}{1+\frac{Q_{21}}{A_{21}}}
$$

shows that the equation giving the Stern-Volmer factor can be re-written as a ratio of the collisional quenching to the fluorescence emission. Since the collisional quenching and the fluorescence emission terms are considered to be an average for all transitions, Hartfield was able to determine the relation:

$$
\frac{Q_{21}}{A_{21}}=c_{q} \frac{p}{\sqrt{T}}
$$

where $c_{q}$ was empirically found to be $2.1 \frac{\sqrt{K}}{k P a}$ for diatomic iodine. $\frac{48}{48}$

\subsubsection{Voigt Function - Line Shape}

The Voigt lineshape function is a convolution of Lorentzian and Gaussian profiles, each of which accounts for the specific contributions of pressure and thermal broadening to the fluorescence signal from the iodine molecule thermodynamic conditions. The Lorentzian profile accounts for the homogeneous pressure broadening, which oc- 
curs when the collisions in the flow field perturb the absorption energy levels. The Gaussian profile accounts for the thermal broadening, which is a non-homogeneous process, resulting from the random thermal motion of the molecules. The motion of the molecules will cause certain molecules to absorb at slightly different wavelengths which results in the broadening of the fluorescence signal. Mathematically the Voigt convolution is written as:

$$
V(B, D)=\frac{B}{\pi} \int_{-\infty}^{\infty} \frac{e^{-y^{2}}}{B^{2}+\left(D-y^{2}\right)} d y
$$

In Equation 3.12, $D$ denotes the tuning parameter, where $B$ is the broadening parameter. The tuning and broadening parameters are defined as:

$$
\begin{aligned}
& B=\sqrt{\ln (2)} \frac{\Delta \nu_{c}}{\Delta \nu_{D}} \\
& D=2 \sqrt{\ln (2)} \frac{\Delta \nu}{\Delta \nu_{c}}
\end{aligned}
$$

In Equation 3.14, $\Delta \nu$ is the de-tuning of laser wavelength from the absorption line center. In both Equations 3.14 and $3.13, \Delta \nu_{c}$ is the collisional linewidth, and is proportional to pressure and inversely proportional to $T^{0.7}$, as shown in Reference 47 . The collisional linewidth can be written as:

$$
\Delta \nu_{c}=C_{b} \frac{p}{T^{0.7}}
$$

The constant $C_{b}$ was determined experimentally to be $4.1962 \mathrm{GHz} \frac{K^{0.7}}{k P a}$, as shown in Reference 46. The Doppler linewidth, $\Delta \nu_{D}$ is defined as:

$$
\Delta \nu_{D}=\sqrt{\frac{8 \ln (2) k T}{m c^{2}}} \nu_{0}
$$

where $m$ is the mass of the molecule, $c$ is the speed of light in a vacuum, $k$ is the 
Boltzmann constant, $T$ is the translational temperature, and $\nu_{0}$ is the molecular center frequency. At high temperature and low pressure, the Doppler broadening, $\Delta \nu_{D}$, will be the dominant mechanism contributing to fluorescence signal broadening. At high pressure, collisional broadening, $\Delta \nu_{c}$, becomes the dominant contributor to signal broadening. For most flows investigated using the PLIIF technique, the flow field will be between both extremes, and therefore a convolution of the Doppler broadening $\left(\nu_{D}\right)$ and collisional broadening $\left(\nu_{c}\right)$ is necessary to accurately account for thermodynamic contributions in the flow field to the fluorescence. The method for solving the convolution utilized in the iodine model is outlined in the work by Humlíčk ${ }^{56}$.

At very low pressure, collisional broadening becomes negligible and the hyperfine structure of the iodine absorption transitions is visible for the overlapping P13/R15 transitions in the iodine absorption spectrum, as shown in Figure 3.51 .

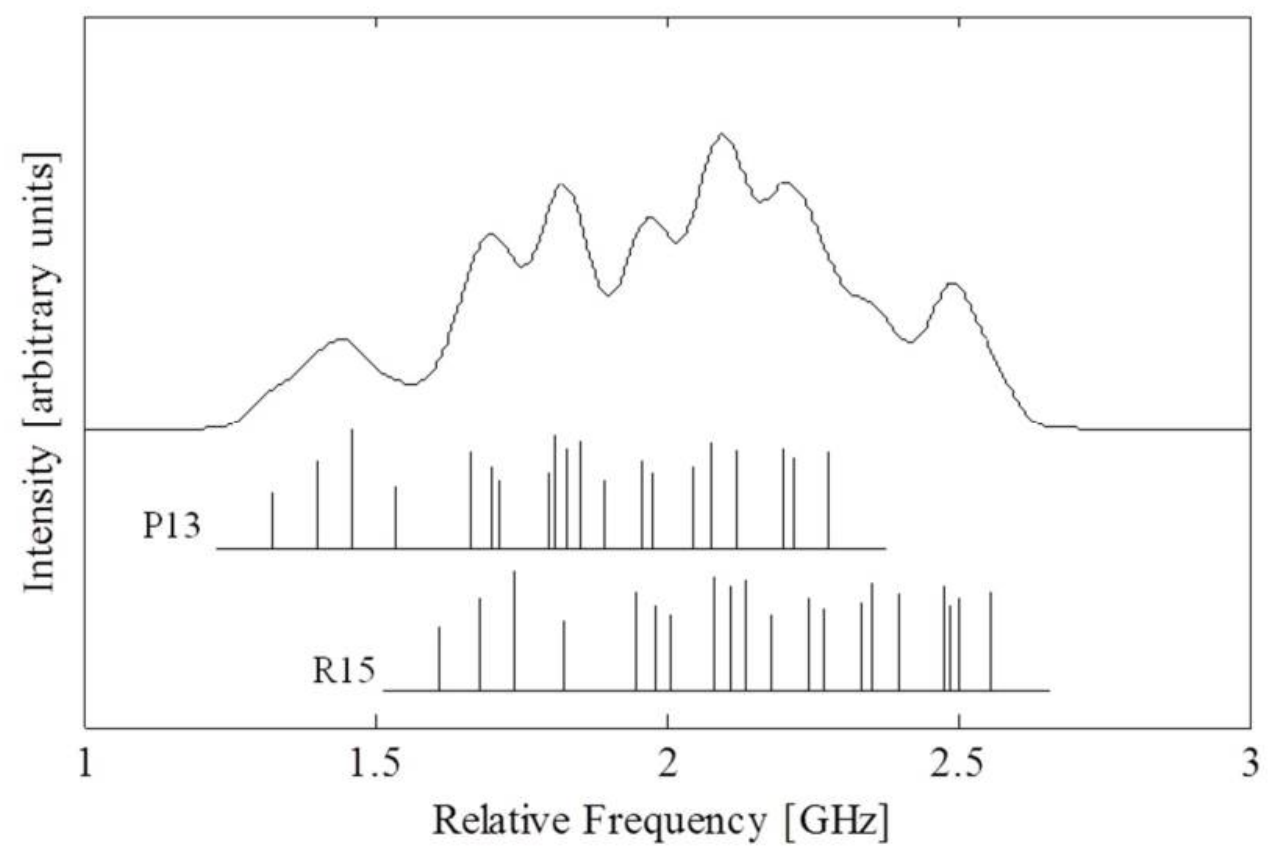

Figure 3.5: Hyperfine spectrum for P13/R15 overlapping transitions in diatomic iodine.

This hyperfine spectrum occurs because of the interaction of the nuclear spin magnetic 
moment with the magnetic field from the electronic spin and orbital angular momentum 57 . The iodine model used in this research contains all hyperfine components in the P13 / R15 transition pair 49 .

The argon-ion gain profile frequency (tuning range) superimposed on the pertinent iodine absorption spectrum at $5145 \AA$ is shown below in Figure 3.6. From Figure 3.6 . it can be seen that the argon-ion laser gain profile spans several pertinent overlapping absorption transitions for diatomic iodine. The nomenclature in Figure 3.6 contains two specific sets of numbers for each transition. For the P13 / R15, the 13 and 15 refer to the values of $J^{\prime \prime}$, while the $(43,0)$ gives the excited and ground state vibrational quantum number $v^{\prime \prime}$, respectively.

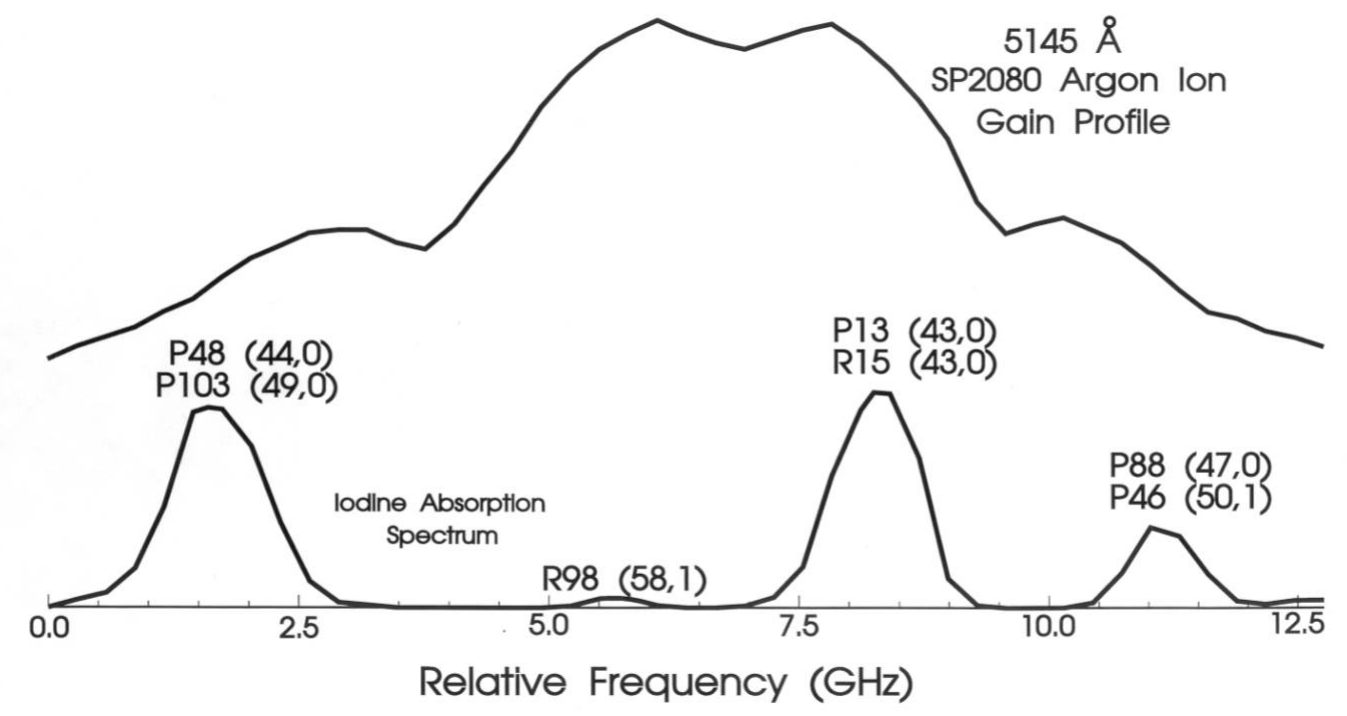

Figure 3.6: Overlay of $514.5 \mathrm{~nm}$ argon-ion gain profile and iodine absorption spectrum.

It can be seen that the P48 / P103 and P13 / R15 overlapping transitions are the highest in relative absorption strength, which makes them both candidates for the PLIIF technique. The R98 transition is known as a 'hot band', due to its high vibrational quantum number, and is not utilized in this research. Also in Figure 3.6. it can be seen that the highest portion of the gain profile coincides with the P13 / R15 overlapping transitions, which makes utilization of these transitions crucial to 
the spectroscopic technique used in this research. By having the strongest portion of the gain profile over the P13 / R15 transitions, the highest fluorescence signal emitted from the seeded flowfield is possible. On the other hand, the location of the P48 / P103 transitions, as well as the P88 / P46 transitions, under the argon-ion gain profile makes them less ideal than the P13 / R15 transitions for the investigations performed for this research.

\subsection{Narrowband Measurements}

The PLIIF technique has the ability to collect quantitative data in both the narrowband and broadband laser emission configurations. The broadband configuration will be covered in the following section. The governing fluorescence equation was shown in Equation 3.3 for the case of narrow bandwidth excitation by the argon-ion laser. A narrowband laser is defined as a laser with laser linewidth less than the absorption linewidth for the molecule being studied. The narrowband laser can be scanned in frequency over discrete iodine absorption transitions, shown in Figure 3.6. in small frequency step sizes. The control of the scanning and single frequency output of the laser is achieved by tilting an intra-cavity air-spaced etalon. The resulting fluorescence emission intensity, as well as laser frequency, are recorded at each discrete step. Subsequently, the fluorescence emission intensity at each frequency can be used to construct an absorption spectrum. Once the absorption spectrum is collected for each pixel in the CCD camera image, a non-linear least squares fit of Equation 3.3 can be applied to the constructed absorption spectra. Since the non-linear least squares fit determines an equation that matches the absorption spectrum, it can be used to calculate the thermodynamic ground state properties from the collected spectra. 


\subsubsection{Velocity Measurement}

In this section a discussion of how velocity can be measured taking advantage of the Doppler effect from narrowband excitation of diatomic iodine will be discussed. The velocity dependence of the PLIIF fluorescence signal is caused by the molecular Doppler shift. This occurs when absorbing iodine molecules interact with the laser radiation at a frequency shifted from the molecular center frequency of the exciting laser, and is due to the orientation of the molecule velocity vector relative to the laser sheet propagation direction. Iodine molecules moving toward the direction of laser sheet propagation will observe the laser radiation at a higher relative frequency, while molecules moving away from the direction of laser sheet propagation will observe the radiation at a lower relative frequency. Iodine molecules moving perpendicular to the direction of laser sheet propagation will not observe a shifted frequency from the molecular center-line frequency. In order to determine the absolute velocity shift for each pixel on the CCD camera, it is necessary to compare the Doppler shifted spectra to the reference frequency obtained in an un-shifted spectrum in a static cell. The entire experimental configuration, including the static cell, will be covered in Chapter 4 .

Once the difference in peak frequency of the static cell spectrum and the flowfield spectrum, obtained by scanning the narrowband laser frequency over the absorption spectrum at $514.5 \mathrm{~nm}$, is measured, the velocity at that specific pixel can be calculated. This is illustrated in Figure 3.7. 


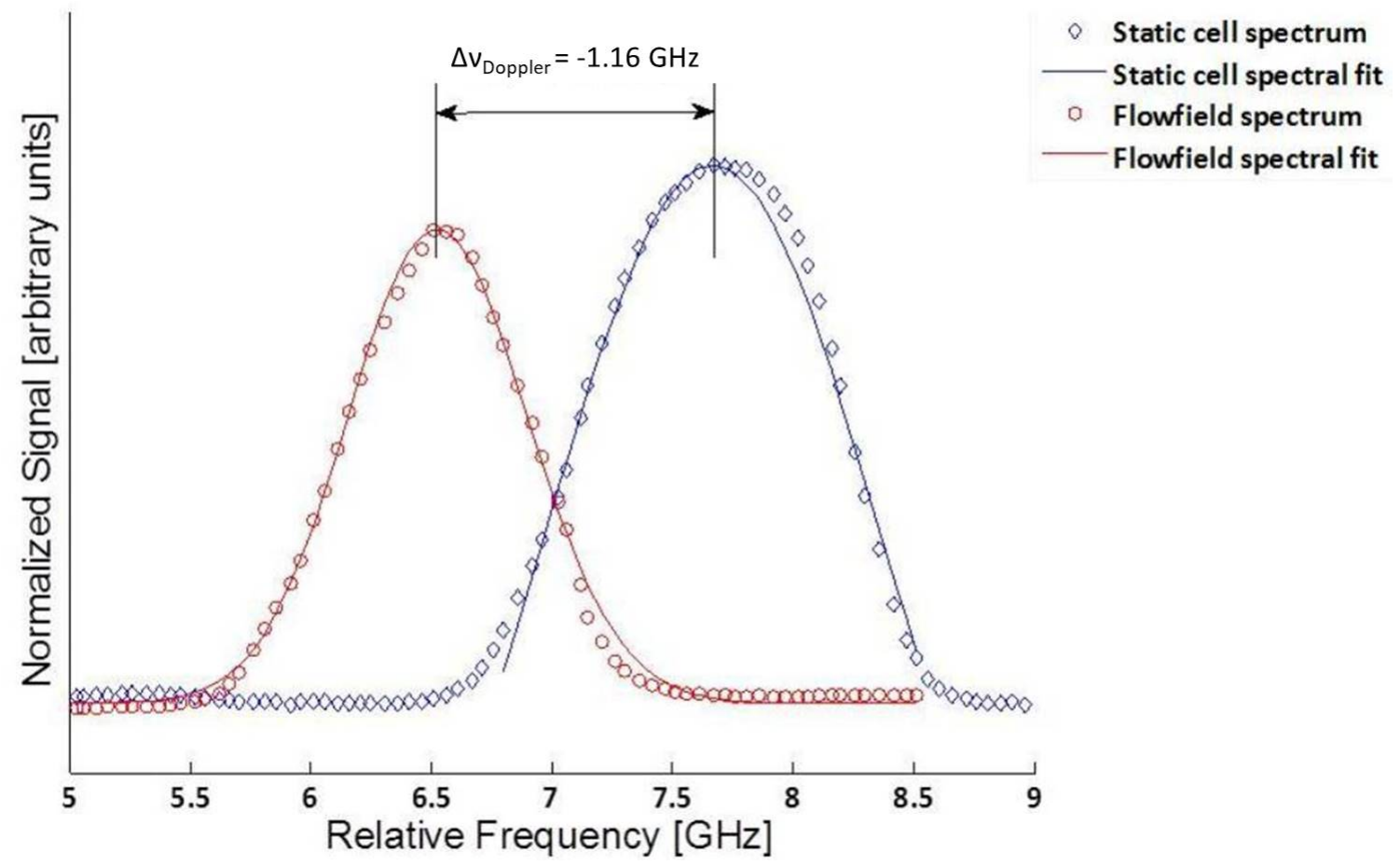

Figure 3.7: Static cell spectrum versus spectrum at a point in the flowfield used to determine Doppler shift.

The Doppler shift equation used to calculate velocity is:

$$
\Delta \nu_{\text {Doppler }}=\frac{u}{\lambda}
$$

where $\lambda$ is the wavelength of the laser. This calculated velocity, $u$, is only for velocity component in the direction of the laser sheet. Therefore, it is necessary to use an additional laser sheet angle on the same flow field to resolve a second velocity component. By measuring two velocity components, and knowing the specific laser sheet angles, a total planar velocity vector at each flowfield location can be calculated.

\subsection{Broadband Measurements}

As discussed previously, another method for data collection using the PLIIF technique utilizes laser emission in the broadband configuration. Broadband refers to the 
simultaneous excitation of all molecular transitions under the laser frequency range, shown in Figure 3.6. This is achieved with the intra-cavity etalon removed. While in this mode of operation, all longitudinal laser modes under the $10 \mathrm{Ghz}$ laser gain profile at $514.5 \mathrm{~nm}$ are excited. This results in the exciting laser linewidth being much greater than an individual iodine absorption linewidth. Due to the emission of all laser modes under the argon-ion gain profile, the Voigt lineshape function, normalized by the Doppler linewidth, integrates to unity. When the normalized Voigt function becomes unity, Equation 3.3 reduces to:

$$
S_{f}=C f_{v^{\prime \prime} J^{\prime \prime}}\left[\frac{A_{12}}{A_{12}+Q(p, T)}\right] I f_{s} n
$$

All variables in Equation 3.18 are the same as in the narrowband version of the fluorescence equation, Equation 3.3. Visualizations and density ratio quantitative measurements rely on the broadband measurement technique, and will be discussed in the following sections.

\subsubsection{Visualization}

Visualization measurements are a useful tool in identifying key features within a flow field, such as shocks and shock interactions, as well as providing direct physical measurement of flow field geometry. In the research discussed here, a hypersonic low density wind tunnel is used for the experimental work. Chapter 4 will cover the

experimental apparatus in depth. It is useful to plot various portions of Equation 3.18 in order to draw some conclusions about applying the broadband laser technique to flow field visualizations. Since the seeding fraction of iodine in the wind tunnel is low, it is sufficient to assume the test gas is strictly nitrogen for these calculations. This assumption means a specific heat ratio of 1.4 can be used to compute the pressure and temperature in the isentropic regions of the flow at various Mach numbers. The 
computed thermodynamic isentropic flow properties can then be used to calculate the Stern-Volmer and Boltzmann population fractions, which will show how these values vary with increasing Mach number in the under-expanded jet flowfield.

Using a wind tunnel reservoir temperature of $300 \mathrm{~K}$, and a stagnation pressure of $280 \mathrm{kPa}$ (the condition in the stagnation vessel where the iodine is seeded with nitrogen), comparisons of Stern-Volmer factor versus Mach number, and Boltzmann population fraction versus Mach number can be computed.

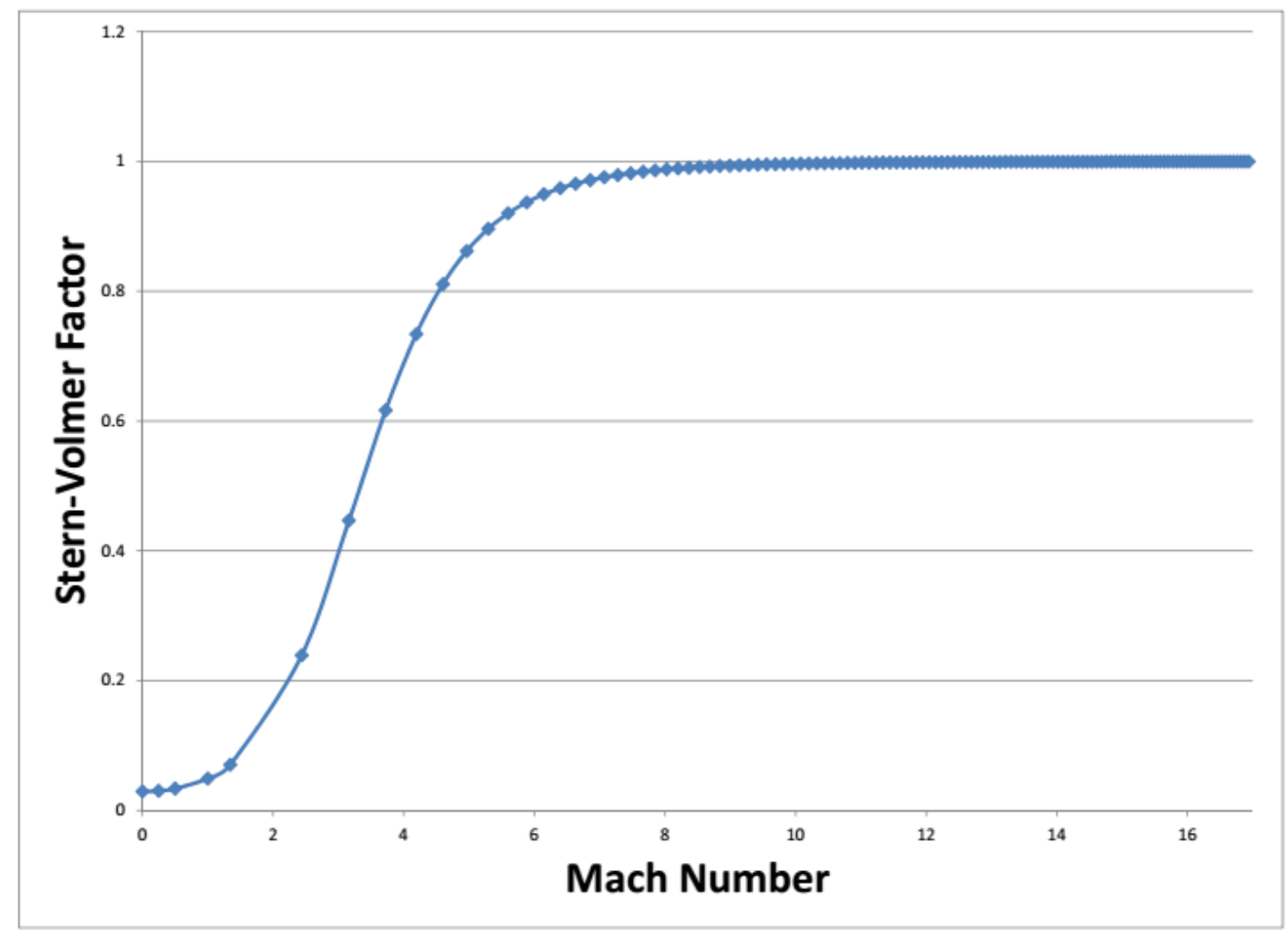

Figure 3.8: Stern-Volmer Factor versus Mach Number.

As the Mach number increases, the temperature and pressure decrease. This results in the collisional quenching term, $\Delta \nu_{c}$, becoming very small. In Figure 3.8 , it can be seen that the Stern-Volmer factor approaches unity by approximately Mach 8.

It is also pertinent to examine the Boltzmann population fraction in this manner. The Boltzmann population fraction versus Mach number is shown in Figure 3.9. 


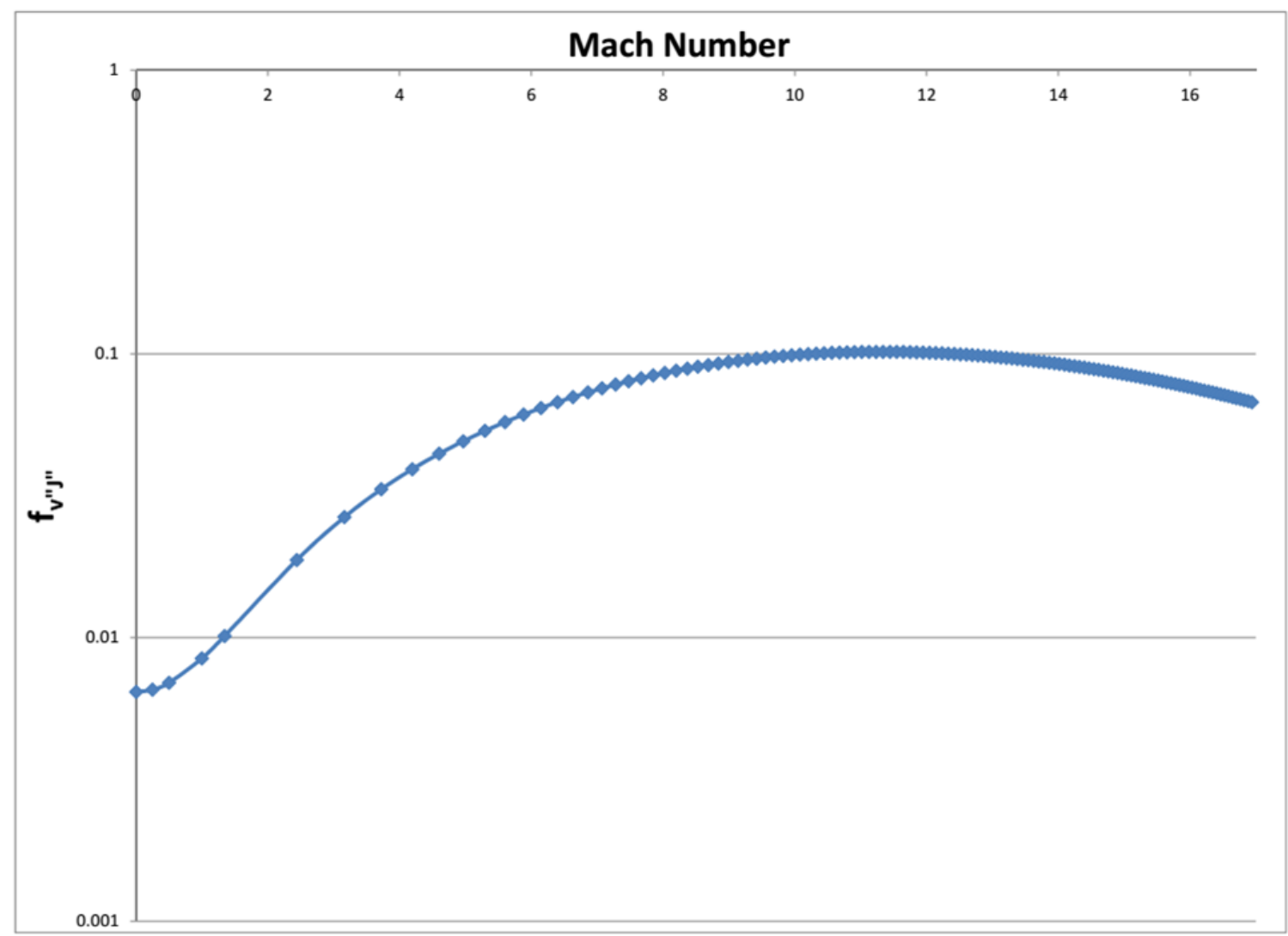

Figure 3.9: Boltzmann population fraction versus Mach number.

Similar to the Stern-Volmer factor, the Boltzmann population fraction is nearly constant between Mach 8 and Mach 14 due to the decreasing flow field temperature. The decreasing temperature results in the rotational population shifting to lower rotational quantum states. Examining the product of the Stern-Volmer factor and Boltzmann population fraction for Mach numbers ranging from zero to sixteen, the constant nature of the product of these parameters, between Mach 8 and 14, can be clearly seen in Figure 3.10 . 


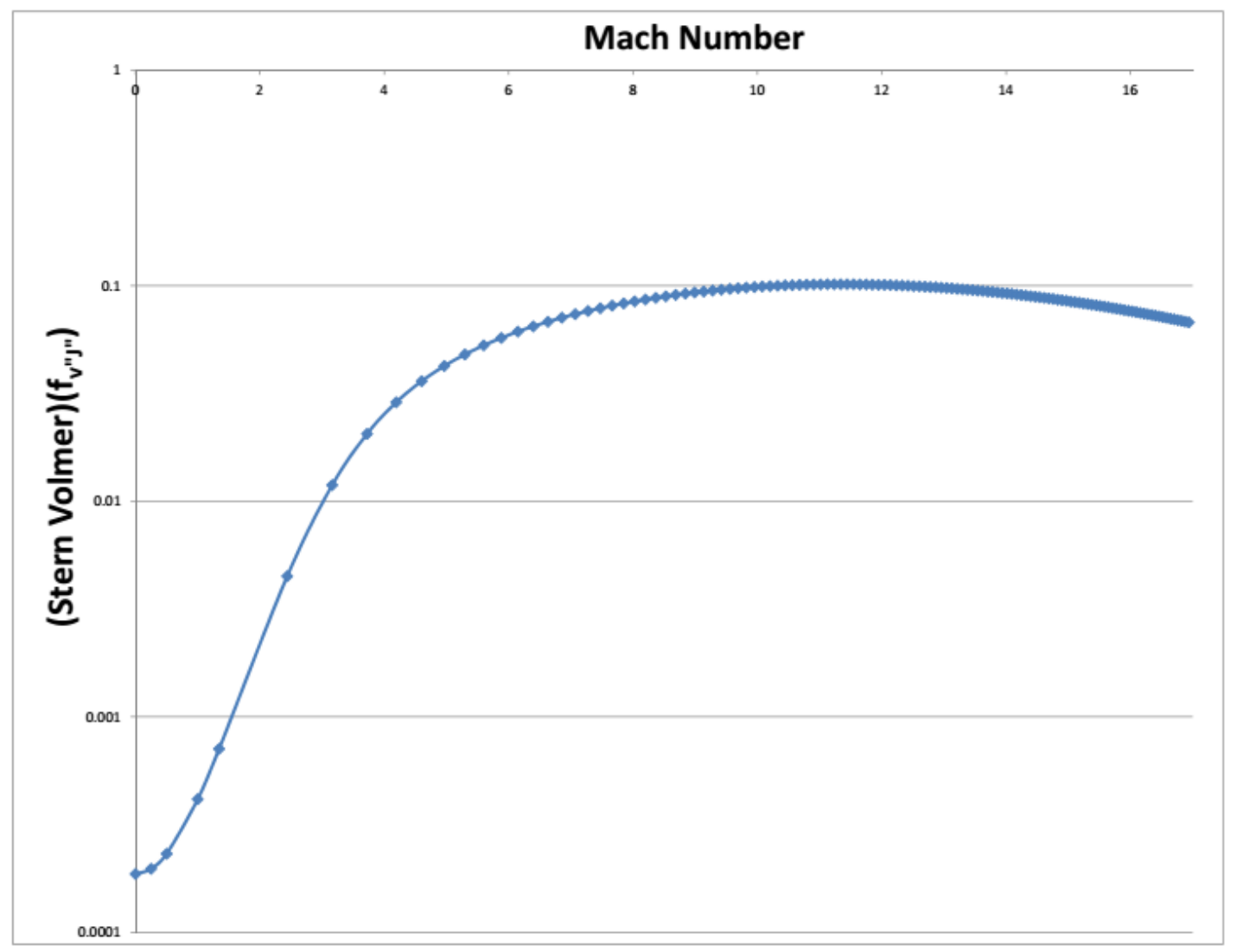

Figure 3.10: Product of Stern-Volmer Factor and Boltzmann Population Fraction versus Mach Number.

If Equation 3.18 is considered with constant laser power, I, and constant iodine seeding fraction $f_{s}$, a Stern-Volmer factor approaching unity, and a Boltzmann population fraction relatively constant at 0.1 , it becomes evident that the fluorescence signal will be directly proportional to the flow field number density over the Mach number range from 8 to 14. Therefore, flowfield density can be measured using broadband PLIIF if the Doppler shift of the broadband fluorescence signal, and actual distribution of the laser power, $I$, can be accurately taken into account.

The Doppler shift created by molecules of iodine travelling in a direction that is counter to the direction of laser sheet causes an increase in fluorescence signal. This is shown by a shift from right-to-left of relative frequency in Figure 3.6. This right-to-left shift of relative frequency observed by the iodine molecule with broadband excitation 
results in a frequency that falls under a higher intensity portion of the gain profile, and will thus cause a bias in the fluorescence signal based on direction of travel of the iodine molecule. Bias is extremely difficult to account for, as it causes non-uniform influence of the fluorescence signal, and for which there is no proven method to remove this bias from the broadband fluorescence data. Experimental methods can be used to align the laser with the flowfield in the most conducive direction possible, which will depend on flow direction and features, but even this will still yield some Doppler bias because not every molecule of iodine will be traveling in the same direction within the flowfield. Chapter 5 contains a discussion on Doppler bias in broadband images.

The ability to quantitatively determine flowfield density from fluorescence signal is useful in a Mach range of 8 to 14 if the laser intensity distribution and Doppler bias are accounted for. Outside of this range, the fluorescence signal depends on the temperature and pressure of the flowfield, which will make any images qualitative in nature.

\subsubsection{Density Ratio}

While the process of accounting for the Doppler bias in the fluorescence signal for a single broadband image is extremely difficult, the ability to measure density within a flow field from a pair of broadband images has been developed and demonstrated previously 5 . By taking the ratio of two broadband images, one with the entire flowfield seeded with iodine and one with only a single jet seeded, a ratio of the single jet contribution to flow field density can be determined. This ratio is a number density ratio, and not a mass density ratio. This ratio will also show how a single jet contributes to any fluid mixing in the flowfield and impingement surface interactions.

The ratio of the fluorescence Equation 3.18, for a single jet seeded and for the entire flowfield seeded with iodine, with a constant seeding fraction, directly results in the ratio of the iodine number density at every pixel (flowfield location). All other 
parameters in the fluorescence Equation 3.18 are identical between the two images and therefore cancel, resulting in Equation 3.19 .

$$
\frac{S_{f \text { SingleJet }}}{S_{f \text { Total }}}=\frac{n_{\text {SingleJet }}}{n_{\text {Total }}}
$$

The ratio of the single jet number density to the total number density becomes related to the ratio of fluorescence signal by the equation:

$$
\frac{n_{\text {SingleJet }}}{n_{\text {Total }}}=A \frac{S_{\text {fSingleJet }}}{S_{\text {fTotal }}}
$$

The scaling constant, $A$, is necessary because all density values calculated in this manner must be scaled proportionally to some known value within the flowfield in order to have meaning. The number density ratio is known to be unity in a region where the flow is completely single jet fluid, so the value of $A$ can be determined by the ratio of the fluorescence signals at that flow field location. This location corresponds to immediately at the jet exit in the core flow of the jet plume. Based on this method, the density ratio can be determined for the contribution of a single jet to the total flowfield, whether that interaction is between neighboring jets or with an impingement surface. 


\section{Chapter 4}

\section{DESIGN OF EXPERIMENT AND EXPERIMENTAL PROCEDURES}

In this chapter the experimental setup, design of the experiment, and the experimental procedure will be covered in depth. The experimental setup will cover the equipment contained in the research lab which make the PLIIF technique possible. The primary pieces of testing equipment consist of the hypersonic wind tunnel, the experimental test section, optical components, and the argon-ion laser. Next, the design of the experimental test section and supporting mounting hardware will be covered. Finally, the process of starting and running a data collection experiment will be discussed.

\subsection{Experimental Setup}

The primary equipment utilized in the research discussed herein is a hypersonic wind tunnel, which is made operational by an in-line series of three vacuum pumps. The vacuum pumps allow for operating background pressures in the hypersonic test chamber as low as 20 millitorr. Supersonic jets in the wind tunnel expand into this low pressure test chamber to produce underexpanded jets with hypersonic flow conditions. The wind tunnel will be discussed in the next section. 


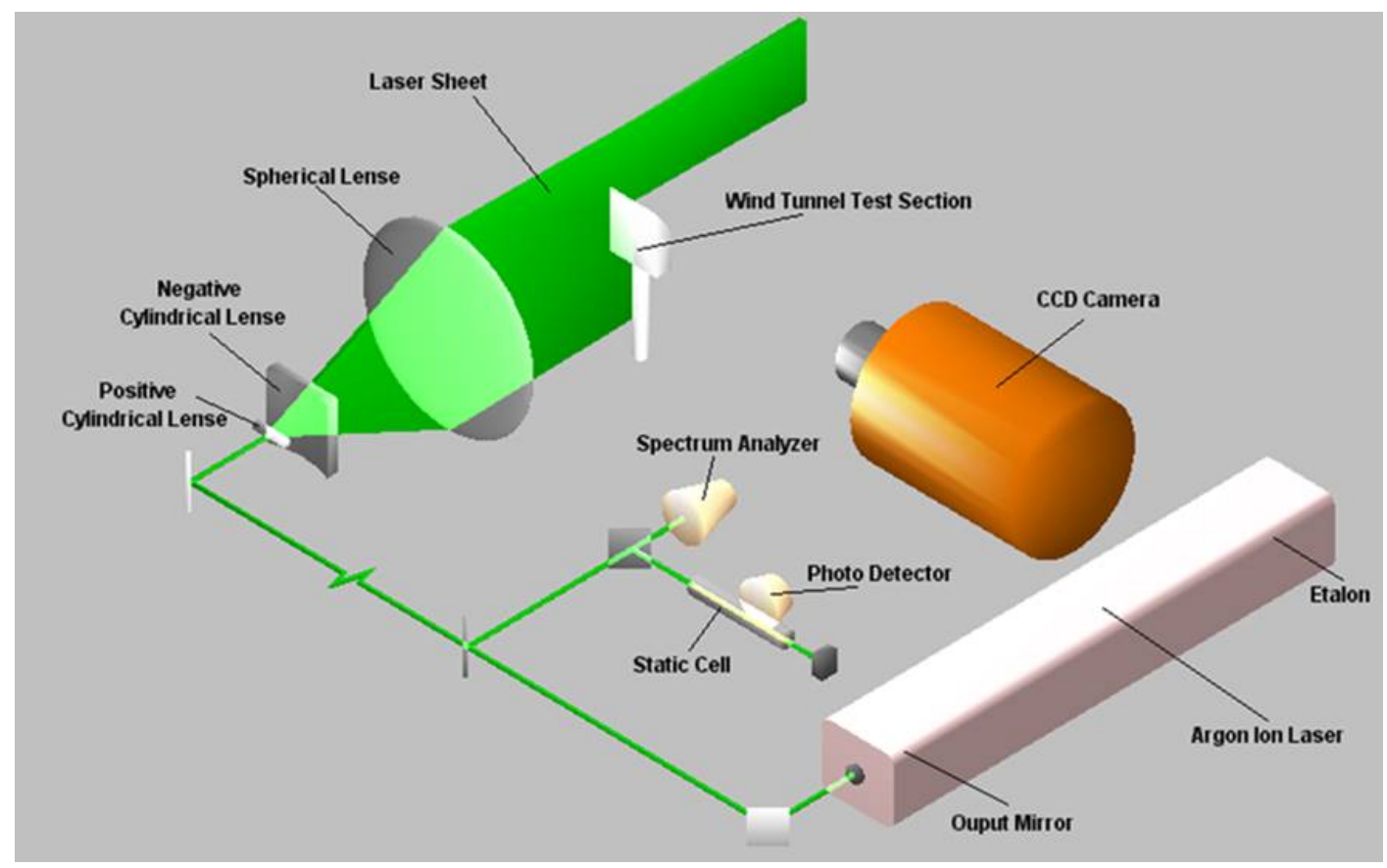

Figure 4.1: Basic experimental layout.

Figure 4.1 shows the optical setup for the PLIIF measurement technique. External to the wind tunnel is a series of optical lenses that transform the cylindrical laser beam into a collimated laser sheet used to excite the iodine molecules seeded into the flow. The laser beam originates from a Model 2080 argon-ion laser produced by Spectra Physics. The laser beam passes through a beam splitter which allows the majority of the laser beam to proceed to the sheet-forming optical array, but a small portion of the laser is sent to a power meter, an iodine static cell, and an interferometer. As part of the laser propagation path, a static cell with stationary molecular iodine is used to create a reference spectrum for iodine at known thermodynamic conditions for narrowband velocity measurement. The static cell is a constant-pressure cell containing iodine vapor at a vapor pressure of 0.3 torr. The spectrum analyzer indicates the relative laser frequency as the laser is tuned through the gain profile, as explained in the narrowband section of Chapter 3. The static cell fluorescence signal is captured by a photo-diode and the electrical signal is processed and correlated to the signal from the spectrum analyzer. In order to capture the fluorescence emission 
from the excited iodine, a charged-couple device camera is mounted perpendicular to the laser sheet propagation direction. The photo-diode, spectrum analyzer frequency, temperature probe signals, pressure probe signals, and camera data are recorded by custom LabVIEW data-logging and control software to create the reference spectrum and associated test data. For the broadband experiments conducted in this research, the static cell, photo-diode, and the spectrum analyzer are not necessary for data collection.

\subsubsection{Hypersonic Wind Tunnel}

The hypersonic wind tunnel facility is a continuous-flow tunnel that provides the formation of under-expanded hypersonic jets in a mixed continuum / rarefied flow regime. For this research, the under-expanded jets being studied result from a higher nozzle exit pressure relative to the back pressure. This pressure differential results in the fluid expanding through a centered Prandtl-Meyer expansion fan once it leaves the nozzle contour in order to match the back pressure. The wind tunnel consists of plumbing used to provide the iodine-seeded test gas into the model test section, and the exhaust of the tunnel to the in-line vacuum pumps. In order to create the flow field for study, nitrogen gas seeded with at a ratio of one part iodine to 7000 parts nitrogen is provided to the test section plenum from an external nitrogen and iodine source. The under-expanded jet plume is a function of the mass flow rate through the nozzle contours and the vacuum system pumping efficiency. The nozzle contour consists of a prescribed contour from the method of characteristic solution, which consists of a throat diameter of 0.5 millimeters, and an exit diameter of 2.5 millimeters, resulting in a Mach 5 exit condition. A picture of the wind tunnel is shown in Figure 4.2. The hypersonic test chamber, in which the low pressure needed to provide the Mach 5 exit condition is contained, is the silver vessel located on the left of the image. 


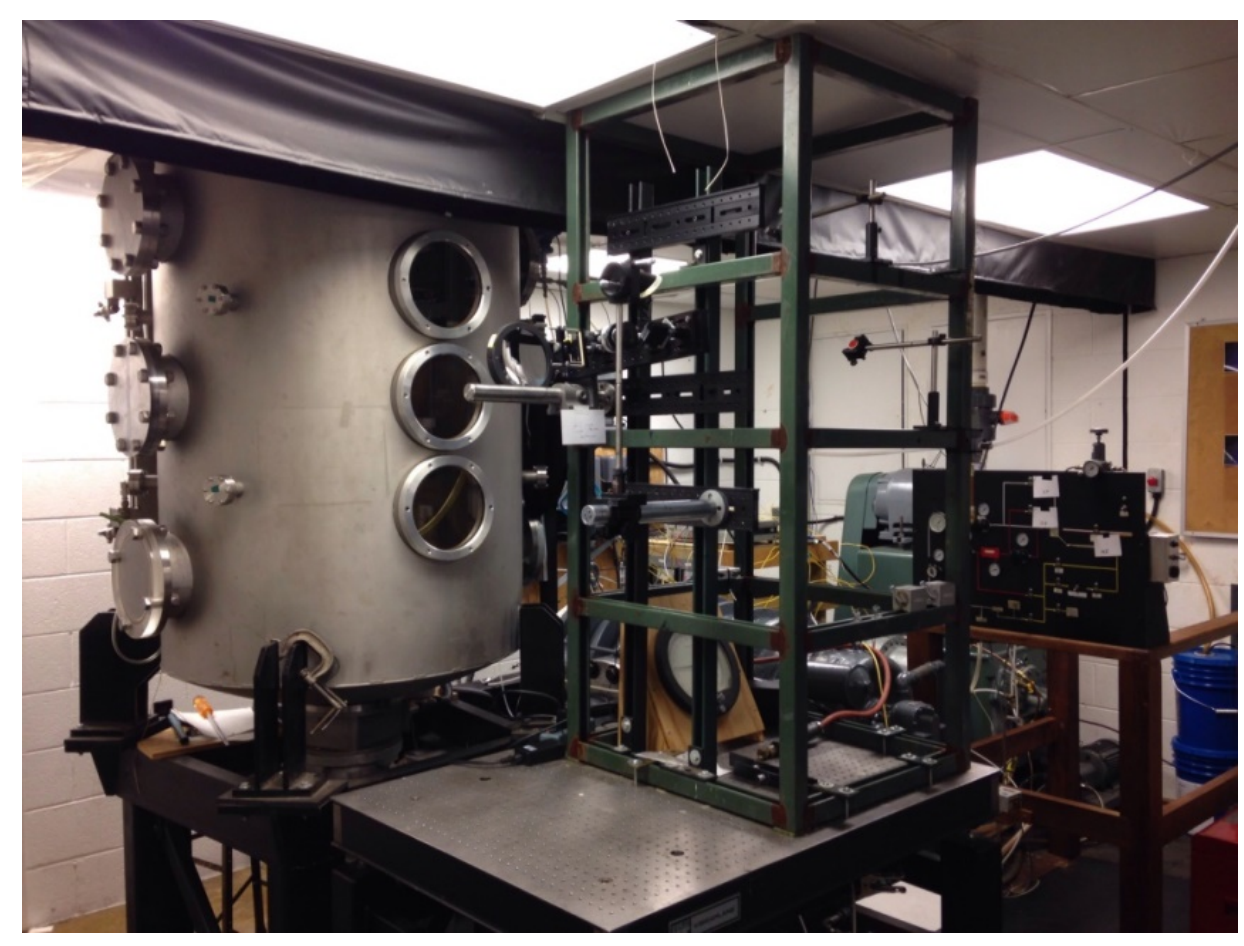

Figure 4.2: Hypersonic test chamber and optics.

Mass flow into the test chamber is created by nitrogen flow to the facility from a bank of eighteen dry nitrogen cylinders located outside of the lab. This volume of dry nitrogen allows for experimental run times up to thirty hours. Nitrogen gas flow from the bank can either flow directly into the iodine mixing vessel, or can bypass the iodine mixing vessel if a flow of only nitrogen is needed for a portion of the flow field, as shown in Figure 4.3. This ability to control the seeding to certain portions of the experimental test section allows for the collection of density ratio quantitative data, covered in Chapter 5 . 


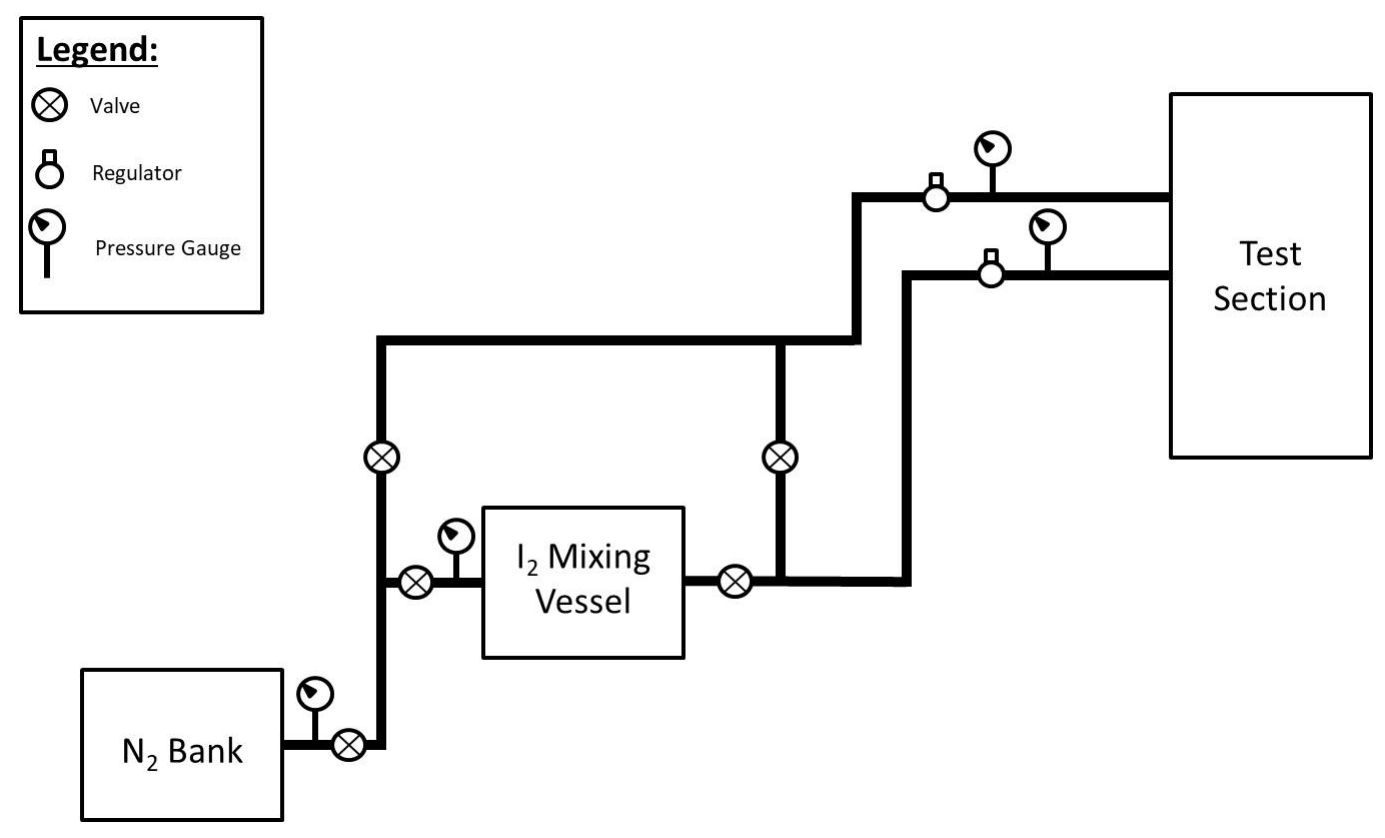

Figure 4.3: Test facility plumbing diagram.

Nitrogen gas that flows into the iodine mixing vessel can be regulated to pressures up to the pressure of the dry nitrogen cylinders, but is typically operated in the thirty to fifty pounds per square inch range. The nitrogen that flows into the mixing vessel mixes with the vapor pressure from solid iodine crystals. The seeding fraction is determined by the ratio of iodine vapor pressure at room temperature and the total pressure of the mixing vessel. The total pressure and temperature in the mixing vessel remains constant for the duration of the experiment.

In order to achieve a constant back pressure in the test chamber during an experiment, the series of vacuum pumps are connected, but vibrationally isolated, from the test chamber vessel. The vacuum system consists of three large vacuum pumps which continuously evacuate the wind tunnel during an experiment. The vacuum pumps consist of a GE-Roots Model 1236 rotary vacuum pump, a GE-Roots Model 615 booster rotary vacuum pump, and a Stokes Model 412 booster piston vacuum pump. Model plenum pressure is regulated by Viton coated plastic needle valves, which allow for consistent, stable operating pressures. Plenum pressure is monitored through two 
electronic pressure transducers and an analog pressure gauge. The electronic pressure transducers consist of a lower pressure model, the Omegadyne Model PX409 0-15 psia transducer, and a higher pressure model, the Omegadyne Model PX409 0-50 psia transducer. The two models allow for accurate measurement of plenum pressure over a wider range of pressures than a single transducer would allow. The temperature of the plenum gas is monitored by a K-type thermocouple connected to a LabVIEW USB thermocouple data-logging interface.

In order to provide laser sheet entry into the hypersonic test chamber, the test chamber has optical access ports arrayed around the circumference of the cylindrical test chamber wall (see Figure 4.2). This allows for optical access for the CCD camera and multiple laser sheet entry angles via the three vertical optical acess ports around the cylindrical test chamber body. Optical quartz windows allow for low-loss laser sheet access into the test section. The remaining unused ports are blocked in order to decrease stray light entering the tunnel from equipment and other light sources within in the lab.

\subsubsection{Optical Systems}

The ports described in the previous section, as well as the optical system, are visible in the center of Figure 4.2. The optical system used to change the cylindrical laser beam into a coherent laser sheet, was illustrated in Figure 4.1. As can be seen in Figure 4.2, the laser beam is turned by multiple mirrors to direct the laser sheet to the hypersonic test chamber.

The cylindrical laser beam is converted into a sheet through a series of three optical components. First, the beam passes through an adjustable iris which blocks the edges from the laser beam, in order to provide a more uniform laser sheet in the experimental test region. Following the iris, the laser beam passes through a -9.7 millimeter cylindrical plano-concave lens. This lens focuses the laser beam horizontally. 
Immediately after the first plano-concave lens is a -400 millimeter plano-concave lens which expands the laser sheet vertically. This lens is followed by a large 330 millimeter plano-convex lens which collimates the laser sheet. The physical spacing of the three lenses are such that the focal points of the first plano-concave lens and the large spherical lens are set to the same spatial location in order to accurately collimate the laser sheet. The -400 millimeter plano-concave lens is used to control the laser sheet thickness at the model, which is typically between 0.2 and 0.3 millimeters in thickness. This is achieved by adjusting the location of the focal point with relation to the large spherical lens. The spacing between the second cylindrical lens and the spherical lens is adjusted to ensure the smallest laser sheet thickness at the test section model location.

\subsubsection{Argon-ion Laser}

The PLIIF technique outlined in Chapter 3 of this work utilizes the 514.5 nanometer wavelength emission of the argon-ion laser to excite the iodine seeded into the flow field. The argon-ion laser used in this work is capable of being operated in two configurations. The multi-mode, or broadband, operation uses the laser in a configuration where all longitudinal modes of the laser under the gain profile are emitted ${ }^{59}$. The narrowband mode is achieved by using an intra-cavity etalon to select individual laser frequencies under the gain profile. The laser is operated in the broadband mode for this research. A figure showing the gain profile and a discussion of the broadband laser operation was covered in the previous chapter.

The laser is operated in a power control mode set to 1.5 Watts output power for both the visualizations and the density ratio experiments. The power control mode is a selectable laser operating mode that continuously monitors the laser output power and makes adjustments to the current provided to the laser head in order to maintain the selected output power. Though the laser is operated in broadband mode for all 
experiments described here, the equipment is present to operate in the narrowband configuration, using an intra-cavity etalon.

\subsubsection{Charge-Coupled Device Camera}

In order to effectively collect the very low level fluorescence emission from the excited iodine molecules, an ultra-sensitive camera is utilized. The camera selected for this research is a 16-bit Andor iKon-L Charge-Coupled Device camera with a 2048 by 2048 pixel resolution. The resolution provided by this model camera is $15.5 \pm 1.0$ pixels per millimeter in the flow area and an image area of 131 by 131 millimeters. The camera has a greater than 95 percent quantum efficiency in the 500 to 600 nanometer wavelength range. In order to maintain such a high efficiency, the camera system utilizes liquid cooling. With internal thermoelectric cooling, supplemented by an external liquid cooling unit, the camera is able to achieve sensor temperatures as low as $-100{ }^{\circ} \mathrm{C}$. This low operating temperature reduces dark current buildup within the CCD sensor chip, which translates to lower noise levels in a given exposure time. The fluorescence signal is further improved by placing an orange Heliopan \#22 filter on the camera optics in order to block scattered laser radiation from the camera lens. This configuration of optics and cooling, paired with the higher performance camera, allows for the collection of very low signal level iodine fluorescence that is common with iodine-seeded gas flowfields.

\subsection{Design of Experiment}

In previous research conducted at the UVA Aerospace Research Lab Flowfield Diagnostics Laboratory, a large under-expanded jet provided the test region. The test chamber was configured as shown in Figure 4.4a. The under-expanded jet test region is shown in Figure $4.4 \mathrm{~b}$, where the left side of the image shows the Mach 
number of the flow in the test region, and the right side shows the corresponding Knudsen number of the flow. In Figure 4.4b, the under-expanded jet exit diameter, $\mathrm{D}$, was $2 \mathrm{~mm}$.

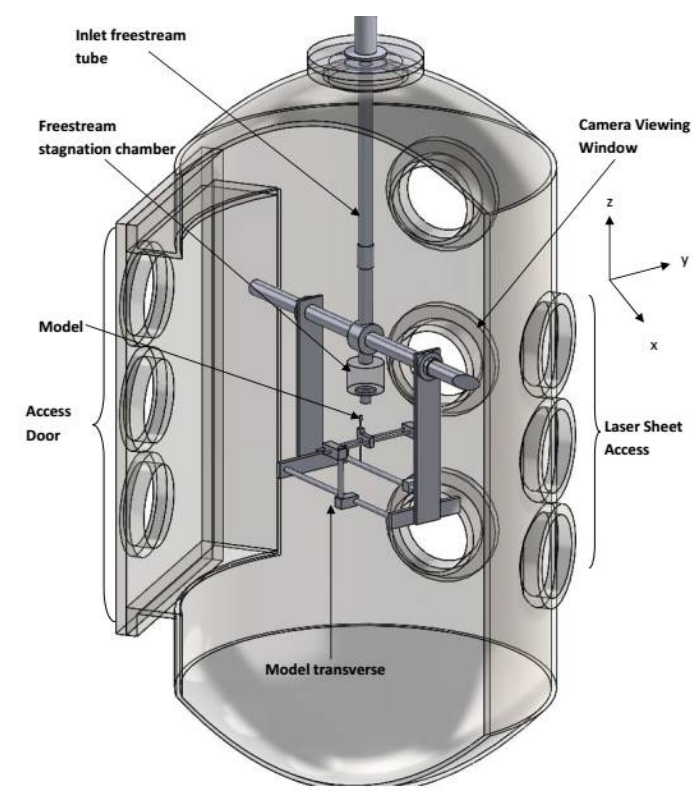

(a) SolidWorks test chamber model.

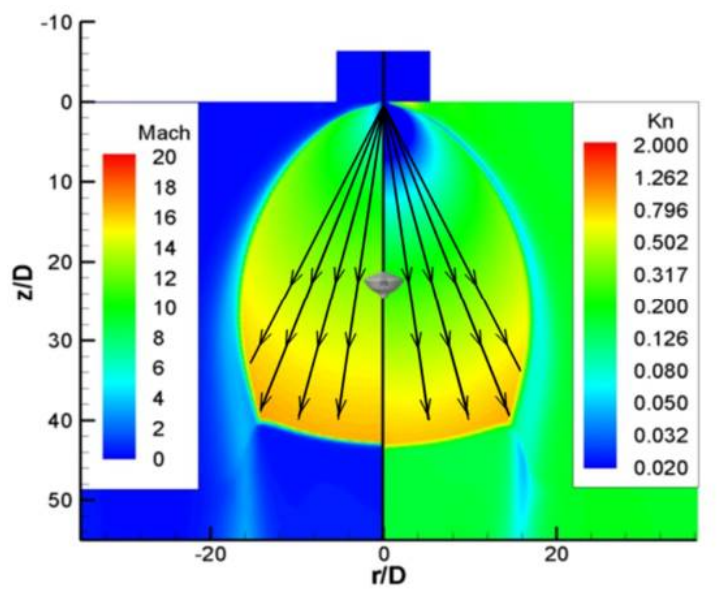

(b) Plot of Mach and Knudsen number in test region.

Figure 4.4: Previous hypersonic test chamber configuration.

The flow accelerated from Mach one at the sonic jet exit to about Mach 16 at the Mach disk location. The Knudsen number approaches one, a very rarefied condition. Models were placed in the test region at locations to give the desired experimental freestream conditions. The models were scaled versions of the Mars Science Lab with either reaction control system jets or retro-propulsion jets.

The nature of the research outlined here demanded an entirely new design for the experimental test section, as well as for the model mounting. Instead of a large under-expanded jet providing the test section, jets issued from a model fitted with a modular stagnation plenum. The modular plenum was designed to provide three different nozzle contour disks which allowed for variable jet spacing. Because the size of the model was significantly larger than previous experiments, and demanded a large degree of adjustability, a robust and flexible design was needed. 


\subsubsection{Model Mounting Design}

The test chamber had limited accommodations for mounting the model within the tunnel. Since the model, which will be covered in-depth in a following section, must contain a gas stagnation plenum with interchangeable nozzle disks, a new design for the model mounting system was needed. In addition, in order to allow for multiple adjustment options in the location of the impingement surface, as well as the model, a new mounting system was designed. In order to allow for multiple designs, the initial design concepts were created using SolidWorks models.

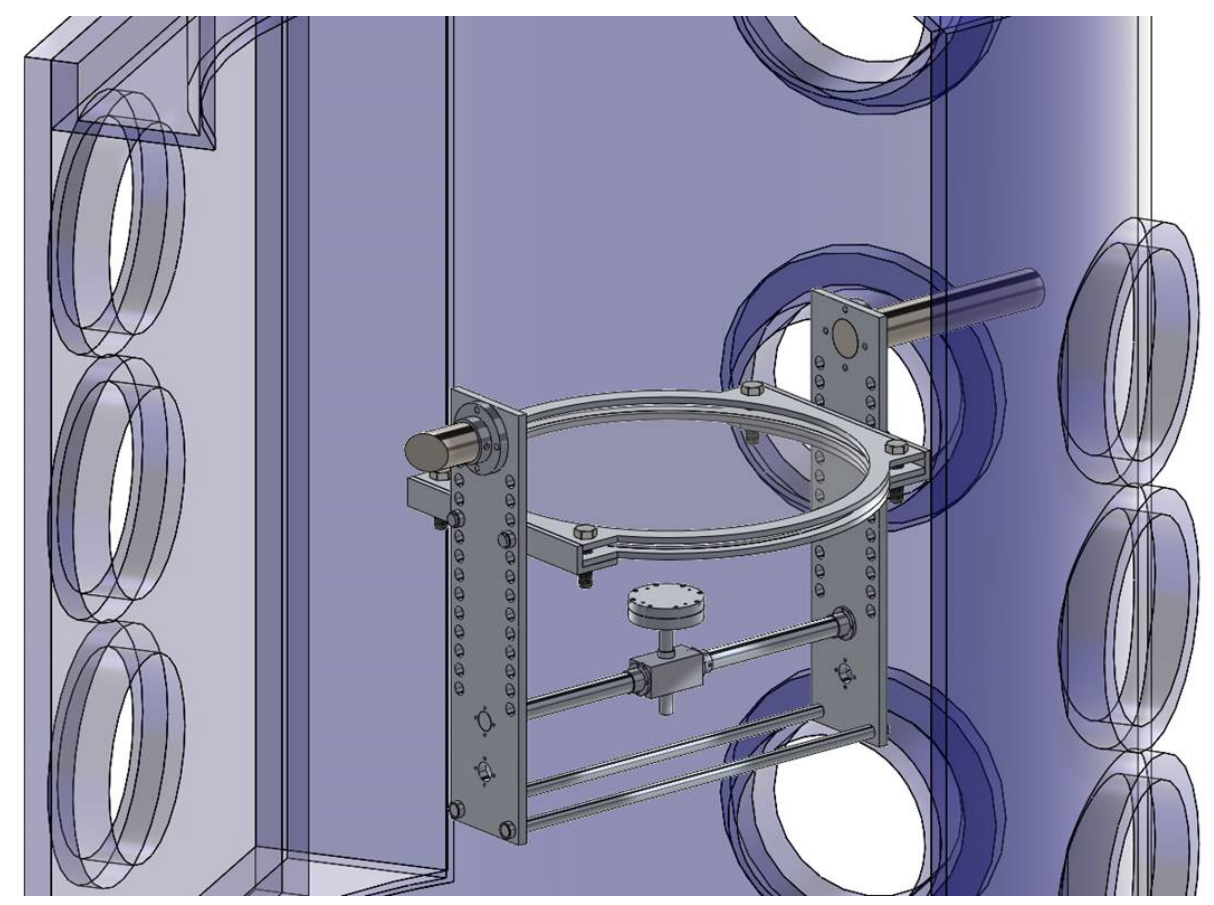

Figure 4.5: SolidWorks representation of the model design.

Within SolidWorks, a dimensionally accurate model of the hypersonic test chamber and associated model mounting points was created. By combining the dimensionally accurate test chamber model with a series of test section mounting designs, a finalized design that was dimensionally precise was developed. The final SolidWorks design is shown in Figure 4.5. In this figure, the adjustable side supports are shown with the parallel holes spaced at half inch intervals, which provides for an adjustment 
range of 0.5 to 6.5 inches of standoff distance between the model surface and an impingement surface. In the center of the image is the model with nozzle disk attached to the stagnation plenum. Above the model, and also in the center of the image, is the transparent impingement surface and retainer plates which hold the impingement surface.

Once the design was finalized, the dimensioned drawings created within SolidWorks were used to machine the model and mounting system. In Figure 4.6, the final model design is shown fitted into the test chamber. This image was taken from the tunnel access door, and shows the final model design and mounting system. In the center of the image, the stagnation plenum with unpainted (aluminum) nozzle disk is shown. In the top center of the image is the impingement surface retainer plates bolted into the vertical supports. The impingement surface is a clear quartz optical glass disk held between the retainer plates. The unpainted metal tubing on the top left and right attaches the mounting system to the hypersonic test chamber walls. This mounting is attached to the chamber walls in a floating configuration to allow for deformation of the chamber under vacuum with minimal interaction with the model mounting system. 


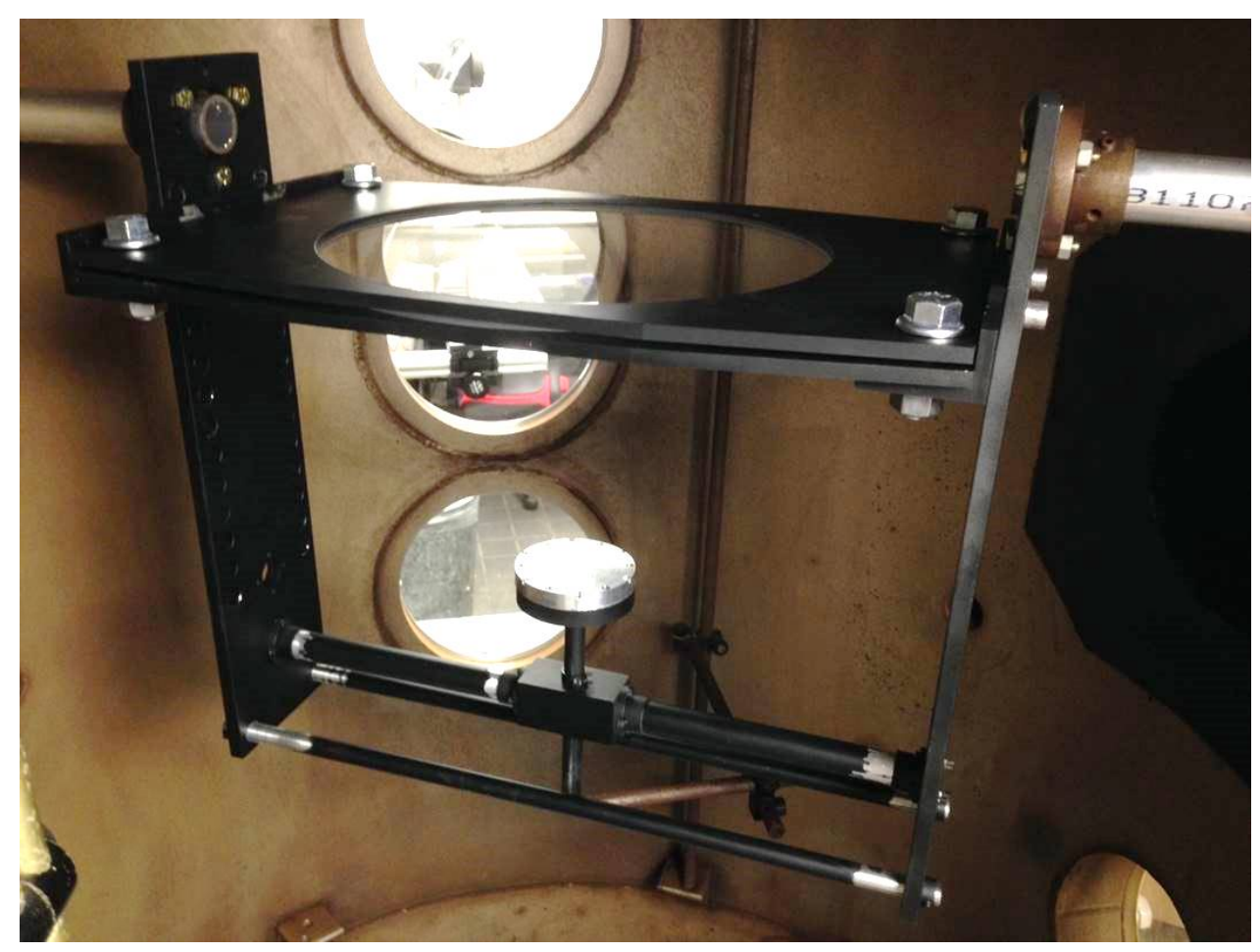

Figure 4.6: Completed model design mounted in the test chamber.

Once the design was completed an initial vacuum test of the model and plumbing was conducted to determine any final design changes that would be necessary to assure proper operation of the design. During the initial tests it was found that an internal baffle was needed to ensure no interference between the vacuum pumping flow, at the bottom of the test chamber, and the iodine-seeded flow field. Figure 4.7 shows the final design configuration used to collect the visualization and density ratio images detailed in Chapter 5 and 6, respectively. Figure 4.7 is very similar to Figure 4.6, except that it is taken from a higher angle in the tunnel access opening. This image also contains the aforementioned baffle, situated below the model in the center of the image, and a painted nozzle disk. The green line in the image is the laser sheet entering the tunnel and aligned to bisect two jet centers on the model surface. The optical quartz impingement surface is also present in Figure 4.7 . 


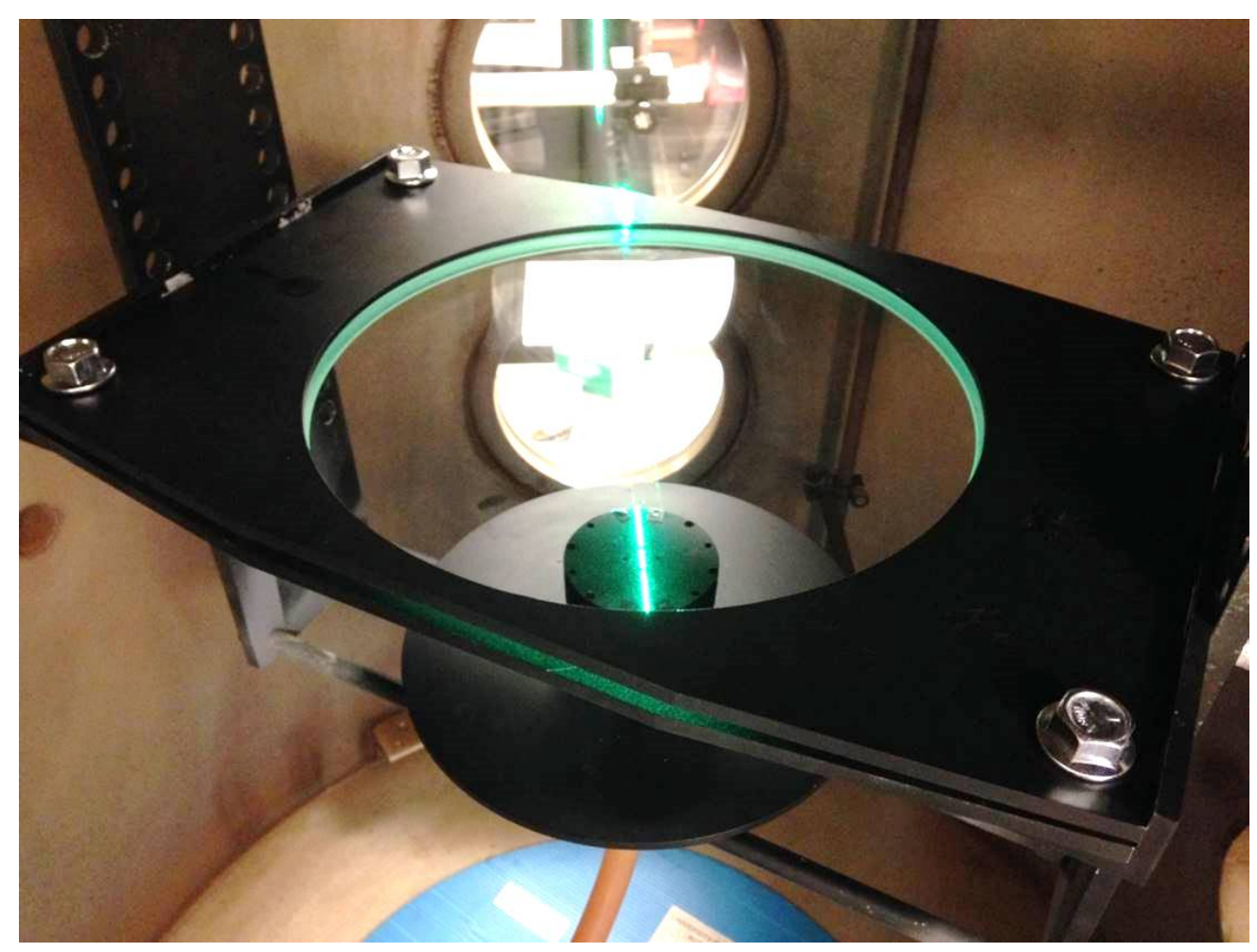

Figure 4.7: Completed experiment design with laser sheet projected on model disk..

A further series of design modifications was needed in order to accommodate broadband density ratio measurements. The addition of a secondary plenum, and associated plumbing, was added to the design shown in Figure 4.7, and will be discussed in the next section. The results of the density ratio measurements will be covered further in Chapter 6 .

\subsubsection{Test Section Design}

The primary focus of the model design was to create a geometry that as closely replicated the geometry of the HTV-3 spacecraft as possible. During the design of the test section mounting system, parallel development of the test model took place. The design of a plenum with modular nozzle disks was selected to allow for simplified changing of model test configurations. Starting from the physical dimensions of the HTV-3 spacecraft, the effort to design geometrically equivalent models was under- 
taken using SolidWorks. The nozzle contour shape was provided by NASA Langley Research Center in order to ensure a Mach 5 nozzle exit velocity. The contour shape was generated with an axisymmetric Method of Characteristics program. Using the supplied nozzle contour, a series of three nozzle disks were designed, as shown in Figure 4.8. Three model designs were selected in order to vary the neighboring jet spacing, shown in the figure by the center-to-center diameter measurement, which would allow for further study of the jet-jet interactions based on spacing. On each model disk, an exterior bolt pattern was included to allow for sealing of the nozzle disk to the stagnation plenum. Table 4.1 shows the pertinent dimensions for the physical spacecraft and the final three nozzle disk designs, labeled Disk 1 through 3 in Figure 4.8.

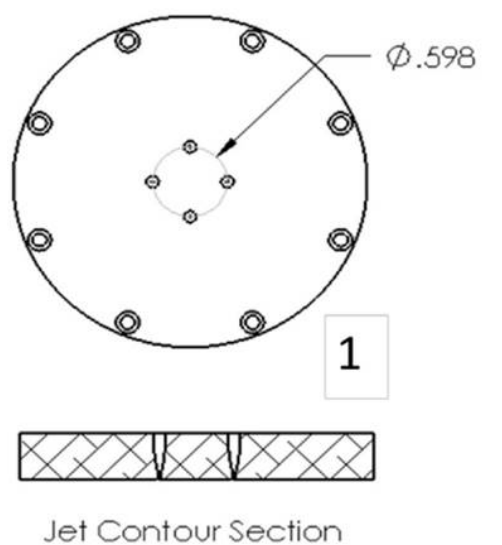

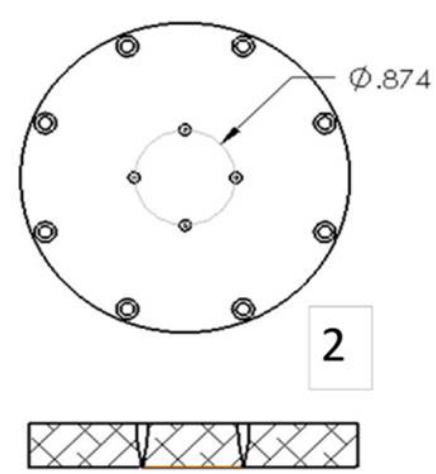

Jet Contour Section

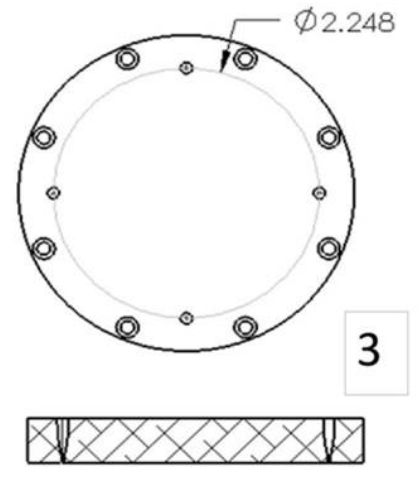

Jet Contour Section

Figure 4.8: Designs for Nozzle Disk.

\begin{tabular}{|c|c|c|c|}
\hline Item & Jet Exit Diameter, $d$ & Center-to-center Jet Spacing, $l$ & Geometric Scaling Factor \\
\hline \hline JAXA HTV-3 & $310 \mathrm{~mm}$ & $1190 \mathrm{~mm}$ & 3.84 \\
\hline Nozzle Disk 1 & $2.5 \mathrm{~mm}$ & $15.2 \mathrm{~mm}$ & 6.08 \\
\hline Nozzle Disk 2 & $2.5 \mathrm{~mm}$ & $22.2 \mathrm{~mm}$ & 8.89 \\
\hline Nozzle Disk 3 & $2.5 \mathrm{~mm}$ & $57.1 \mathrm{~mm}$ & 22.8 \\
\hline
\end{tabular}

Table 4.1: Pertinent physical dimensions.

In the process of selecting the finalized design dimensions, the focus of the research 
into jet-jet interactions was taken as the primary design criteria. In order to ensure that center-to-center jet spacing maintained a significant amount of interaction, a geometric scaling factor was calculated for each design. The geometric scaling factor used in the test section design is defined as:

$$
\text { Geometric Scaling Factor }=\frac{l_{\text {jet-jet }}}{d_{\text {jet exit }}}
$$

In Equation 4.1, $l_{\text {jet-jet }}$ represents the center-to-center jet spacing, shown as the measurements included in Figure 4.8. The diameter of the jet exit, $d_{\text {jet exit }}$, is the denominator of Equation 4.1. The geometric scaling factor for each nozzle disk design is included in Table 4.1. The smallest scaling factor is about 50\% larger than the equivalent HTV-3 scaling factor. A model with the exact geometric scaling of HTV-3 was not used in this research due to machining limitations driving the final dimensions on Disk 1.

Before final design selection and machining, a first order calculation was performed to determine the approximate jet plume boundary dimensions based on a method outlined by Latvala and Anderson 30 . Figure $4.9^{30}$ illustrates the Newton-Raphson solution method used to solve the continuity and conservation of energy equations between any two points, $n$ and $n+1$. The solution method follows a similar process to the axisymmetric irrotational method of characteristics solution discussed in Chapter 2. The solution script was run using initial conditions of: $P_{\text {ratio }}=18805$ and $\theta_{n}=0.15$ for an under-expanded single jet. The value for $P_{\text {ratio }}$, calculated as the ratio of plenum pressure to back pressure, was selected based on the experimental stagnation pressure and back pressure collected during vacuum system calibration runs. The stagnation pressure was set to 30 psia, and the corresponding back pressure was expected to be 82.5 millitorr. The nozzle exit angle, $\theta_{n}$, was selected to be slightly more than zero to account for possible machining error. 


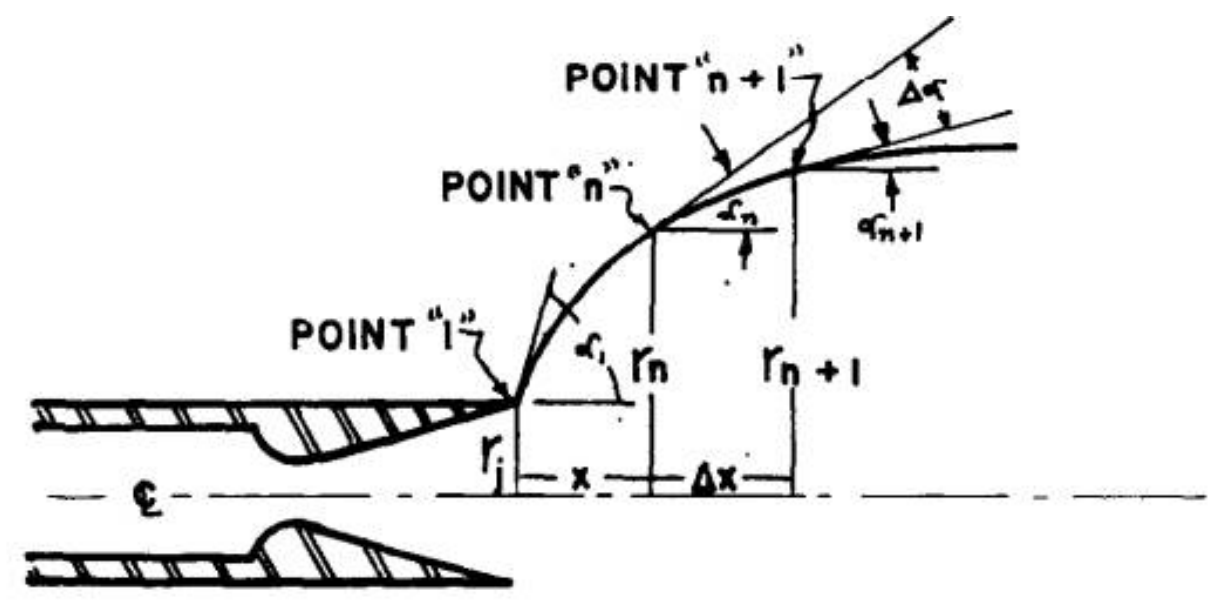

Figure 4.9: Jet Boundary Solution Method from Latvala and Anderson.

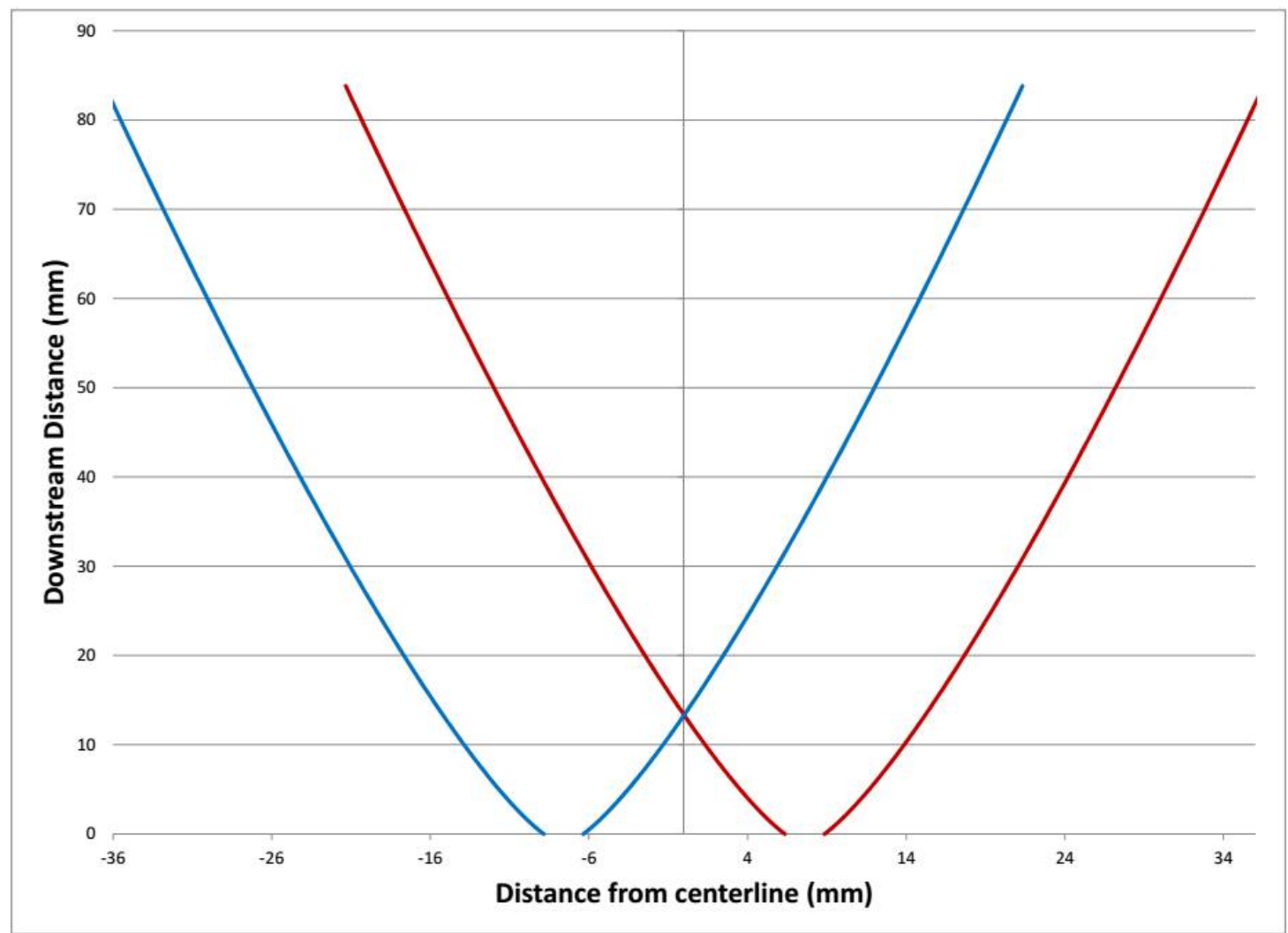

Figure 4.10: Jet Interaction Indicated by Boundary Prediction for Nozzle Disk 1.

The numerical solution was used to determine the predicted location of neighboring jet boundaries, which informed the neighboring jet spacing dimensions in order 
to achieve significant jet interactions. Using the solution with $P_{\text {ratio }}=18805$ and $\theta_{n}=0.15$, the jet boundaries were plotted based on a jet center-to-center distance of 15 millimeters, corresponding closely to nozzle disk 1. Figure 4.10 shows the solution to the jet boundary prediction, plotted in order to determine the interaction of the neighboring jet. The predicted jet boundary interaction was used in the selection of model jet center-to-center dimensions. It was decided that Disk 1 would give significant jet-jet interaction and closer spacing was not necessary.

After machining the nozzle disks and conducting the experiments described in Chapter 5, the predicted interaction location, shown in Figure 4.10 was compared to experimental data. Creating an overlay of Figure 4.10 with experimental jet-jet interaction data, an assessment of the validity of the prediction can be made. Figure 4.11 contains the result of this comparison.

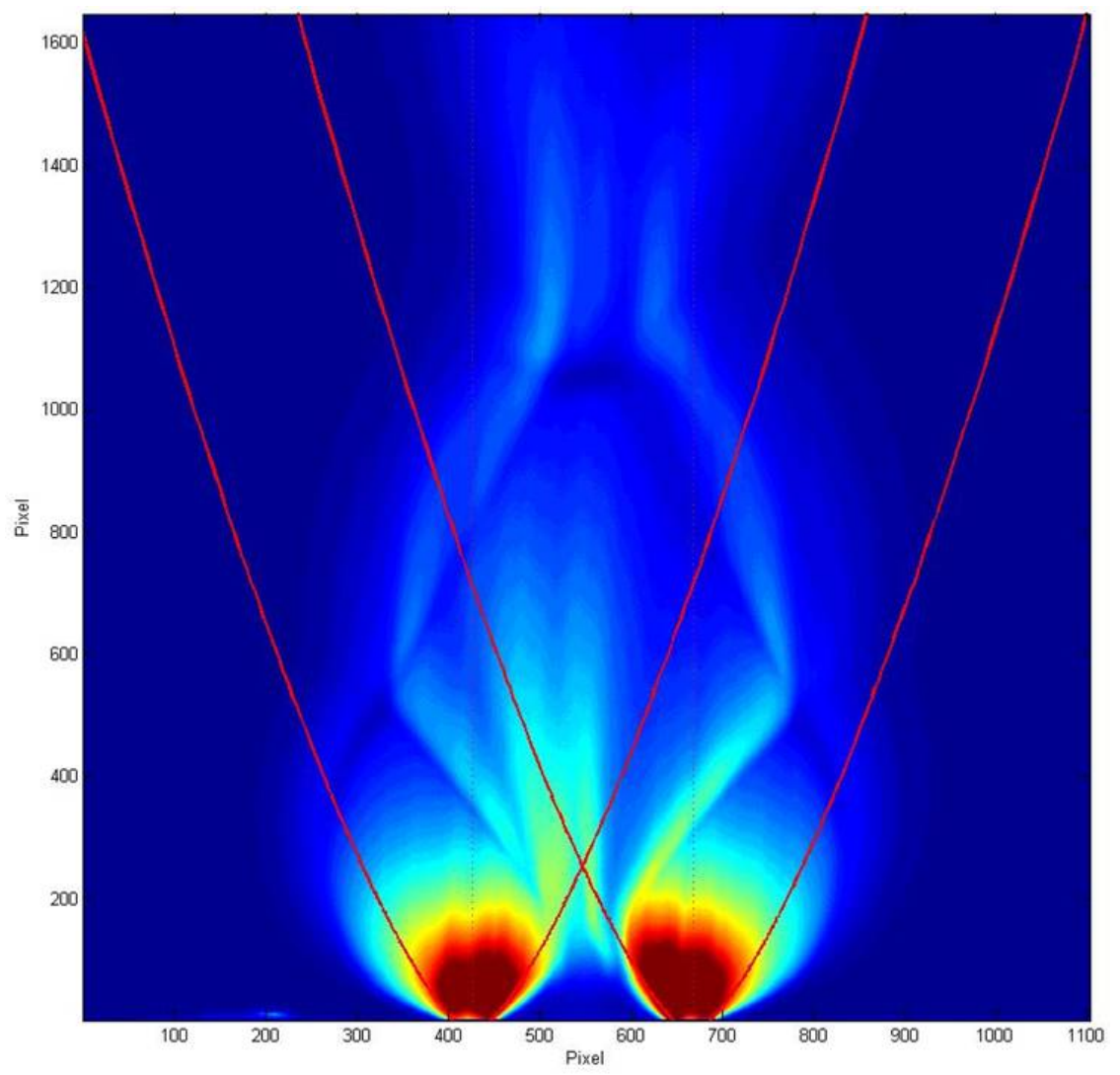

Figure 4.11: Nozzle Disk 1 Experimental Interaction versus Prediction. 
In Figure 4.11, the experimental data shows the point of intersection between neighboring jets occurring approximately $2.8 \frac{x}{D}$ above the nozzle disk surface, where $\frac{x}{D}$ refers to the ratio of downstream location, $x$, to the jet exit diameter, $D$. The Latvala solution method predicted an interaction at $5.6 \frac{x}{D}$ above the nozzle disk surface. The maximum jet boundary width predicted was $10 \frac{w}{D}$, where $\frac{w}{D}$ is the jet width normalized by the jet exit diameter, and the experimental single jet maximum width was $8 \frac{w}{D}$. Based on this comparison, a good agreement between the predicted and experimental maximum jet width can be seen. The predicted location of interaction also shows good agreement, and for the purpose of informing the design of the nozzle disk dimensions, it proved an accurate prediction.

Based on the jet boundary calculations and machining restrictions, the geometric scaling factor provided by Nozzle Disk 1 was chosen to be most equivalent to the HTV3 spacecraft. Nozzle Disk 1 is approximately 50\% larger in geometric scaling factor, which means that Nozzle Disk 1 may exhibit slightly less neighboring jet interactions than the actual spacecraft, but will provide significant interaction. The jet boundary predictions indicate that the results from experiments using Nozzle Disk 1 will be very similar to that of the HTV-3. Since the main objective of these experiments is to provide data to be replicated with DSMC modeling procedures, an identical geometric scaling factor was not strictly necessary. The two other center-to-center jet spacing disks were also machined in order to further quantify the changes in neighboring jet interactions as center-to-center distances increased.

In Figure 4.12, an exploded view of the entire model assembly clearly shows the plenum design and sealing methodology. In the exploded view, the bolts, nozzle disk, and Viton O-ring are shown above the stagnation plenum. The plenum is shown, with the mounting sting removed, at the bottom of the image. The stagnation plenum is an open cavity that allows the incoming nitrogen and iodine to stagnate at a controlled pressure prior to exhausting from the nozzle contours. 


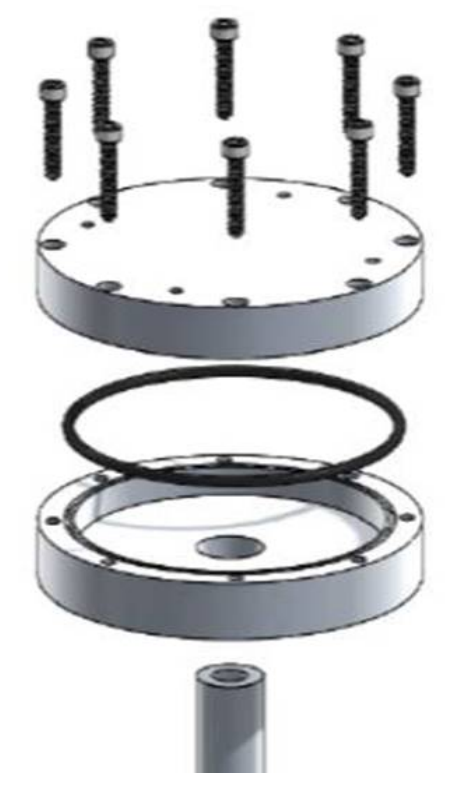

Figure 4.12: SolidWorks Exploded Model Assembly.

An example of a final machined nozzle disk, with the top of the disk on the left and bottom of the disk on the right, is shown in Figure 4.13. In this figure the nozzle exits can be seen to the inner side of the exterior bolt holes, and surrounded with a red circle. In the right portion of the image, the nozzle entrances are found in locations corresponding to the nozzle exits in the left portion of the image, and surrounded by green circles.

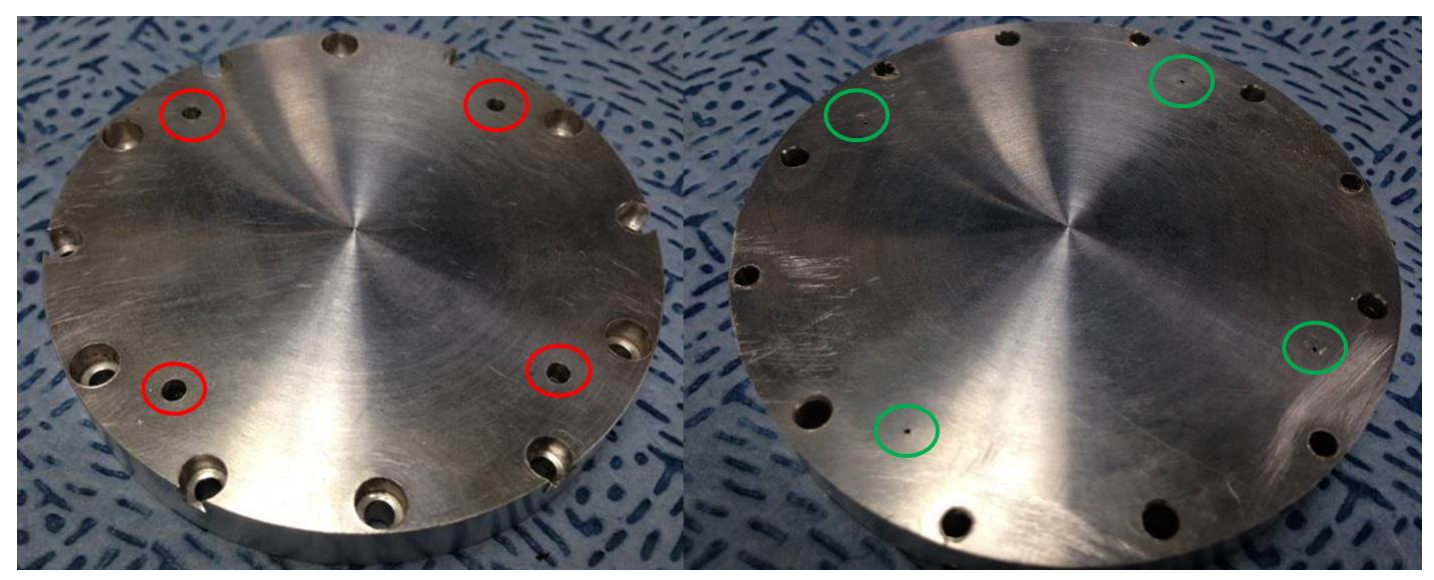

Figure 4.13: As-machined Nozzle Disk 3.

All three nozzle disks were investigated in this research, and in a variety of im- 
pingement surface standoff distance configurations. The results from these investigations will be covered thoroughly in Chapter 5 .

\subsection{Experimental Procedure}

In this final section of Chapter 4, the experimental procedure used to conduct the research investigations into jet-jet interactions and impingement surface interactions is outlined. This section will include an operating procedure for the lab equipment, as well as a general outline of how the visualization and quantitative density ratio images are collected. This experimental procedure section will not cover the operation of the PLIIF technique and equipment in the narrowband, single frequency scanning method, as that method was not an objective of this research.

To begin an experiment, the supporting equipment is brought on-line. The facility cooling water pump is started, which is used to cool the vacuum pumps. The cooling water is cycled through a Filtrine cooler unit. The Filtrine cooling unit further cools the facility water and mitigates any thermal energy introduced to the facility water loop during the experiment. Once this equipment is operating, the NESLAB System III cooling unit is started, which provides the 'clean water'cooling flow to the laser head. Once adequate flow rate and no-load operating temperature have been confirmed using mechanical flow meters and K-type thermocouples respectively, the argon-ion laser is switched on. The laser is initially started in Current Control mode, and set to an operating current that provides an output power of approximately 0.5 Watts. While the laser in warming up, the CCD camera is initialized along with the National Instruments thermocouple monitoring software. Once the monitoring of process temperatures is established, the initial start-up phase is complete.

While the equipment outlined above is reaching thermodynamic equilibrium, the model is inserted into the wind tunnel and configured in whichever experimental con- 
figuration is desired. The model is then aligned with the CCD camera using custom alignment dowels placed on the jet exits. These custom dowels, which measure 1.5 millimeters in diameter and are made of soft plastic, ensure correct alignment of the model with the camera, which is extremely important when bisecting two interacting jets with a laser sheet. Once the model alignment to the camera is confirmed, the initial laser sheet alignment can be completed. An initial rough laser sheet alignment is completed at this stage, which will be further refined prior to data collection. At this point, the test chamber is sealed and the vacuum pumps are started.

Once the vacuum pumps are engaged, the pumps are allowed to warm up to proper operating conditions prior to beginning the flow of liquid nitrogen. The pumping efficiency of the three in-line vacuum pumps is such that an operating back pressure of 60 to 80 millitorr is possible. By using liquid nitrogen flowing through a baffle at the vacuum exit to the wind tunnel, the pumping efficiency can be increased and a back pressure of 20 millitorr becomes possible (with no working gas flow into the test chamber). The liquid nitrogen cooled baffle also acts as a low temperature sink that attracts the condensation of free iodine within the tunnel. The condensation also serves to remove iodine before it is exhausted to the environment. This liquid nitrogen condensation, coupled with a charcoal scrubber, ensure that no iodine is exhausted into the atmosphere during the experimental run.

Once the pumping system has reached operating thermodynamic equilibrium, and an equilibrium flow of liquid nitrogen has been established, the working fluid can be introduced into the wind tunnel through the model test section. Once the nitrogen and iodine flow has been introduced into the wind tunnel, the vacuum system will take a period of time to achieve mass flow equilibrium. Once mass flow equilibrium has been established, the data collection can begin.

If conducting a qualitative broadband visualization experiment, the CCD camera exposure time is adjusted until the fluorescence signal is just below sensor saturation. 
Then a single jet image can be captured which shows the flow field geometry and general geometric dimensions of the flow field. If conducting the broadband planar density ratio experiment, which will be covered in depth in Chapter 6, the flow field is first established with all jets (entire flow) seeded with iodine and nitrogen. The exposure time for the CCD camera is established in the same manner as the qualitative visualization process. This image with all jets seeded with iodine is one of two necessary for the planar density ratio method. Leaving all control valves set at the all jets (entire flow) seeded image settings, the flow to the three jet plenum is switched to nitrogen only. After waiting a period of time for the iodine seeding fraction to drop to acceptable levels within the three jet plenum, an image can be taken with all jets flowing, but with a single jet seeded with iodine with the same exposure settings as the fully-seeded image. It is necessary to wait for a period of time following a switch to unseeded flow in the three jet plenum because the gas present in the plumbing and three jet plenum will have residual iodine present until a sufficient volume of nitrogen can purge the residual iodine from the flow path. This comprises the second image needed to create the density ratio quantitative measurement. The results for qualitative visualizations and quantitative planar density ratio measurements are covered in Chapter 5 and 6 . 


\section{Chapter 5}

\section{Results - Broadband Visualization}

\subsection{Disk 1 Qualitative Results}

Based on the design of the HTV spacecraft and the nature of the flight incident, a complex test matrix was developed in order to determine which experimental configurations most accurately simulated the ISS incident. Three nozzle spacings were initially selected in order to categorize the impact of jet spacing on both the jet-jet interactions and the impingement surface interactions. For the research presented here, the results from the jet spacing most dimensionally similar to the HTV spacecraft are presented first. Testing was also conducted with the other neighboring jet spacing disks, Disk 2 and 3, and the results from these experiments are presented following the discussion of the Disk 1 results.

The development of the test matrix resulted in the selection of two primary jet configurations and two flow configurations. The selections consisted of four-jet and two-jet configurations, with free exhaust into a vacuum, and impingement on a flat surface in a vacuum for flow configurations. For all experiments shown here, the jets exit normal to the model and impingement surface at Mach 5. For these experiments, pressure ratio is defined as:

$$
P_{\text {ratio }}=\frac{\text { Plenum Pressure }}{\text { Back Pressure }}=\frac{p_{o}}{p_{b}}
$$

All data discussed in this chapter was collected using the broadband technique, 
outlined in Chapter 3. With the laser emitting at all frequencies under the argonion gain profile, the raw images collected using this technique contain a bias in the fluorescence intensity, known as the Doppler bias. It is worth re-iterating, from Chapter 3, that a velocity vector opposing the laser sheet direction will result in the molecules 'seeing' an increase in laser frequency. Therefore the laser frequency must be decreased in order to be resonant with the same rotational-vibrational level. This results in the P13 / R15 absorption line to be shifted towards the center of the gain profile. This shift towards the center of the gain profile yields a larger laser power interaction with the molecule pertaining to that velocity vector. Conversely, a velocity vector with lower magnitude in the direction of the laser sheet, whether from a lower velocity magnitude or a flow direction less aligned with the laser sheet direction, will have less shift towards the center of the gain profile. The lower shift will result in a lower fluorescence intensity. This shift, either towards or away from the center of the gain profile, gives rise to the Doppler bias observed in broadband fluorescence data. In order to demonstrate the Doppler bias of the fluorescence signal, a test was conducted where the direction of travel of the laser sheet was varied at two equidistant angles from vertical for the same experimental conditions. In the experiment, the laser sheet was oriented at $-11^{\circ}$ and $+9^{\circ}$, relative to vertical, in order to capture the change in signal intensity with the change in direction of travel of the laser sheet. Figure 5.1 contains the fluorescence intensity for the same flowfield, where only the laser sheet angle-of-attack has been altered. 


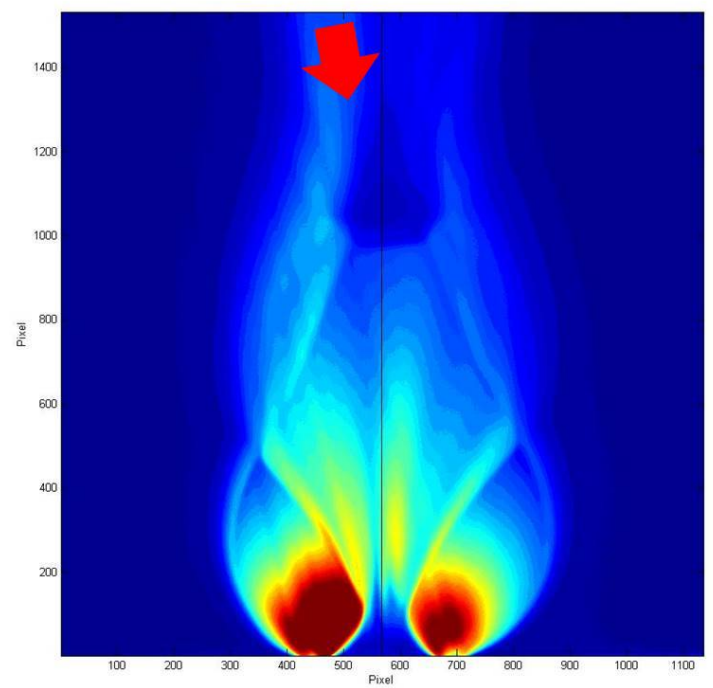

(a) Four Jet Free Exhaust $-11^{\circ}$ From Vertical.

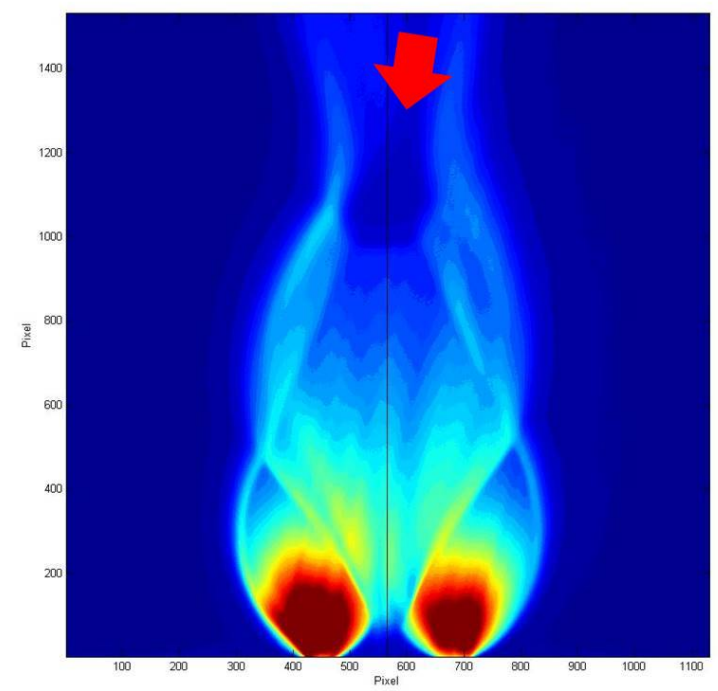

(b) Four Jet Free Exhaust $+9^{\circ}$ From Vertical.

Figure 5.1: Visualization of Doppler Bias in Broadband Images.

Figure 5.1a shows higher fluorescence intensity in the left jet core, along with the left boundary flow, while the right jet core and boundary flow has lower fluorescence intensity. It can also be seen that the fluorescence intensity in the jet core has a bias in the left-to-right orientation, which corresponds to the direction of travel of the laser sheet. The bias manifests in a higher jet core fluorescence, as well as 'streaking' of the fluorescence signal in the direction of laser sheet travel. The 'streaking' is due to the manifestation of the hyperfine absorption spectrum of the iodine molecule, and will be discussed later in this chapter. As the angle-of-attack of the laser sheet is changed to $+9^{\circ}$, Figure $5.1 \mathrm{~b}$ shows the fluorescence in the right jet core increase, along with the right boundary flow fluorescence. It can also be seen that by switching the laser sheet angle of attack, the 'streaking' of the fluorescence signal in the jet core has changed orientation to align with the new angle of attack. In this image, the bias in the jet core has shifted to a right-to-left orientation, which aligns with the angle-of-attack of the laser sheet. The two images in Figure 5.1 demonstrate the Doppler bias in broadband fluorescence images experimentally. 
Since the Doppler bias is inherent in all broadband PLIIF images, the experimental data appears to show an asymmetry in the flowfield, which is not physically present. The asymmetry rises from the Doppler bias influencing the fluorescence intensity in certain portions of the flow. In order to create symmetric images, a mirror technique is utilized. The mirroring technique consists of separating the broadband image into two portions, one to the left of the model centerline, and one to the right. The data with the greatest fluorescence is selected and then mirrored around the model centerline to form a symmetric image of the flow. This technique is used to provide a qualitative visualization of the flow, negating the Doppler bias in the image, and creating a symmetric image that is indicative of the real flowfield geometry. All images presented in Chapter 5 are presented using the mirroring technique, and have also been trimmed to only show the flow from the surface of the nozzle disk to the upper edge of the camera field-of-view, and from the left and right edge of the nozzle disk.

The first experimental configuration investigated was the four jet, free exhaust configuration. This experimental setup simulates the jet plume exhaust interaction from the HTV spacecraft prior to maneuvering within the vicinity of the ISS during docking procedures. This configuration is primarily an investigation of the neighboring jet interaction. It utilizes Disk 1, which has a jet center-to-center spacing of 0.598 inches, or $6.1 \frac{x}{D}$. As discussed previously, $\frac{x}{D}$ refers to the ratio of downstream location, $x$, to the jet exit diameter, $D$. 


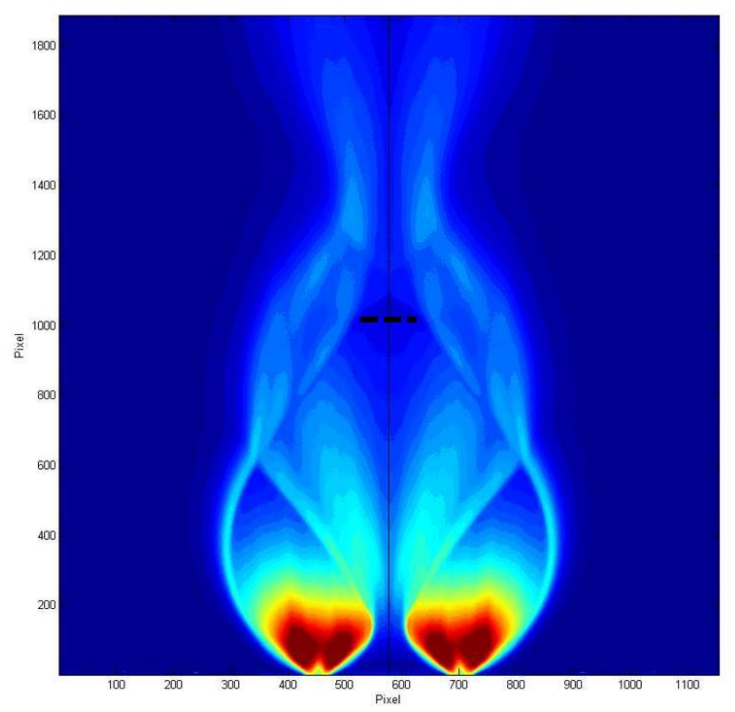

(a) Linear Contour Plot.

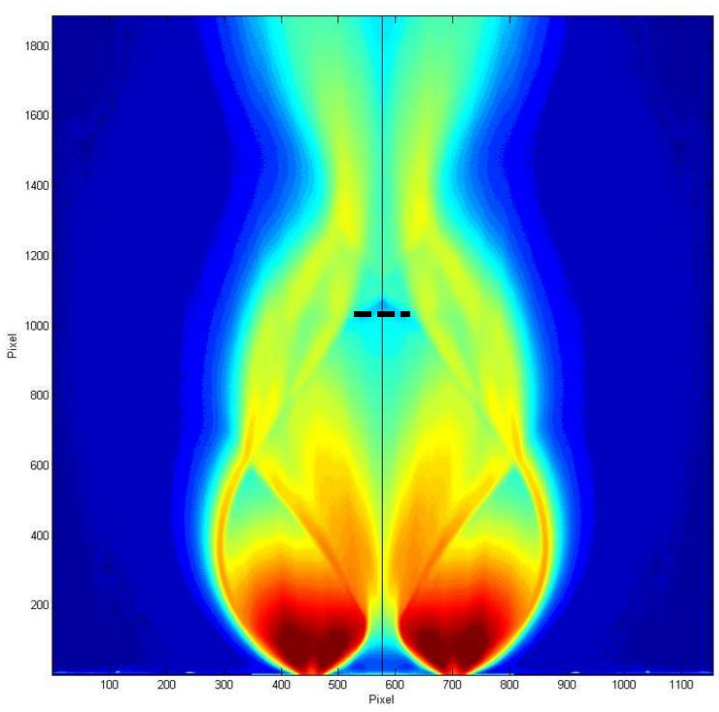

(b) Logarithmic Contour Plot.

Figure 5.2: Disk 1 - Four Jet Free Exhaust Fluorescence Plots.

For this experiment a plenum, or stagnation, pressure of 40 psia was used for the jets. This plenum pressure resulted in a vacuum chamber back pressure of 238.8 millitorr, which resulted in a $P_{\text {ratio }}$ of 8663 . The image in Figure 5.2 has been post-processed in Matlab using background subtraction to remove scattered laser light. Figure 5.2 contains a linear contour plot of fluorescence signal, as well as a logarithmic plot of the fluorescence signal which allows the lower signal regions to be clearly visualized. In the free jet experimental image, distinct jet-jet interactions are present, and the flow field is much more complex than the DAC model calculations predicted. In Figure 5.2, the distinct interaction of the jets is evident immediately downstream of the jet exit. Using a criteria of a florescence signal of $15 \%$ of the maximum fluorescence as the edge of a jet plume, the width and location of interaction can be determined. The initial interaction takes place at $2.7 \frac{x}{D}$ from the nozzle exit. This interaction causes the jets to take on a distinct shape from the deflection of the neighboring jet. Further downstream from the interacting region, an area of lower signal is evident immediately prior to flow approaching the model center line, indicated by the black line in the figure. This low density region is located at $26.8 \frac{x}{D}$ downstream from the 
nozzle exit. This decrease in fluorescence signal is due to an increase in collisional quenching due to increased pressure, which indicates a shock structure in the flow. Approximately mid-way downstream from the jet exit, the flow exhibits a distinct broadening of the barrel shock resulting from the interaction of the neighboring jets. The maximum width for the flow region is $16.3 \frac{x}{D}$.

In the following images, Figure 5.3, an optical glass impingement surface was placed 3.62 inches, or $36.8 \frac{x}{D}$, from the top surface of the model to create flow field interaction with a surface. The plenum pressure was again set to 40 psia. The back pressure was lower than the previous four jet experiment due to the interaction of the impingement plate and the pumping efficiency of the vacuum pumps. The resulting back pressure was 199.3 millitorr, which resulted in a $P_{\text {ratio }}$ of 10379 . Within this image a white line was added to mark the bottom of the impingement surface for reference. A distinct normal shock can be seen to have developed in the flow field, as well as an outward deflection of the hypersonic jet plumes. The initial interaction of the neighboring jet occurs at $2.8 \frac{x}{D}$, and the flowfield maximum width is $16.8 \frac{x}{D}$. The normal shock is marked in the figure with a dashed black line, and occurs at $22.4 \frac{x}{D}$ downstream of the nozzle exit. The normal shock and low fluorescence signal regions are much clearer in the logarithmic plot in Figure 5.3. Again, the lower fluorescence signal downstream is due to the pressure rise across the normal shock and subsequent increase in collisional quenching. 


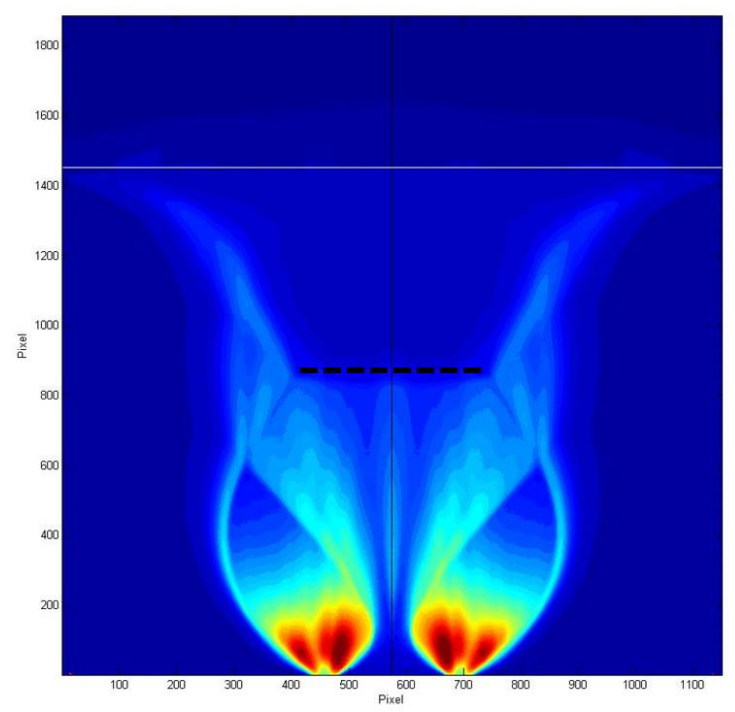

(a) Linear Contour Plot.

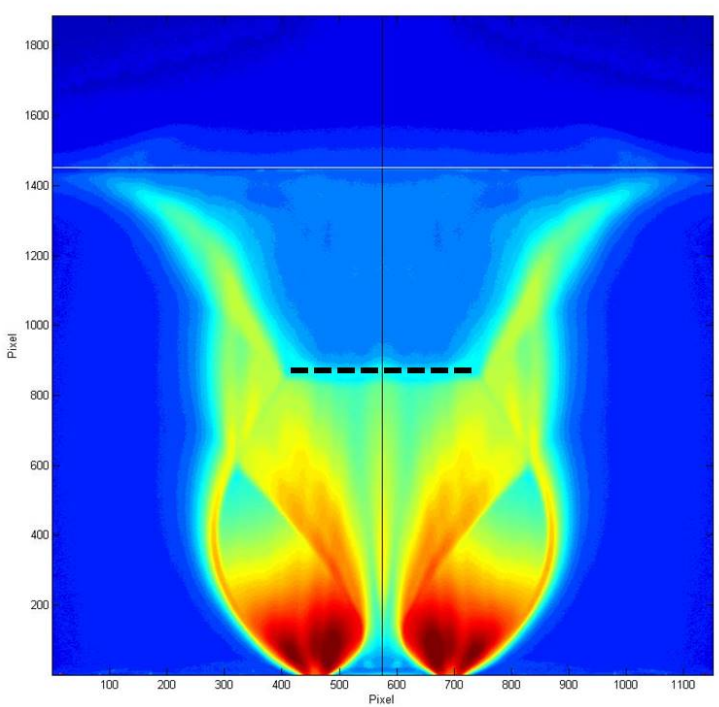

(b) Logarithmic Contour Plot.

Figure 5.3: Disk 1 - Four Jet Free 3.62" Impingement Fluorescence Plots.

In order to further determine the effect of the impingement surface standoff distance, another experiment was conducted that used a closer impingement standoff distance. In this experimental configuration the impingement surface was moved from 3.62 inches to 2.875 inches. This new standoff distance equates to a $29.2 \frac{x}{D}$. Again, the plenum pressure was set to 40 psia and the operating back pressure was 210.9 millitorr. Based on Equation 5.1, the $P_{\text {ratio }}$ is 9808 . The back pressure was lower than the four jet free exhaust experiment due to the interaction of the impingement plate and the pumping efficiency of the vacuum pumps. As with the previous four jet impingement images, a white line was added to mark the bottom of the impingement surface for reference. A distinct normal shock can be seen to have developed in the flow field, as well as an outward deflection of the hypersonic jet plumes. The initial interaction of the neighboring jet occurs at $2.9 \frac{x}{D}$, and the flowfield maximum width is $16.4 \frac{x}{D}$. 


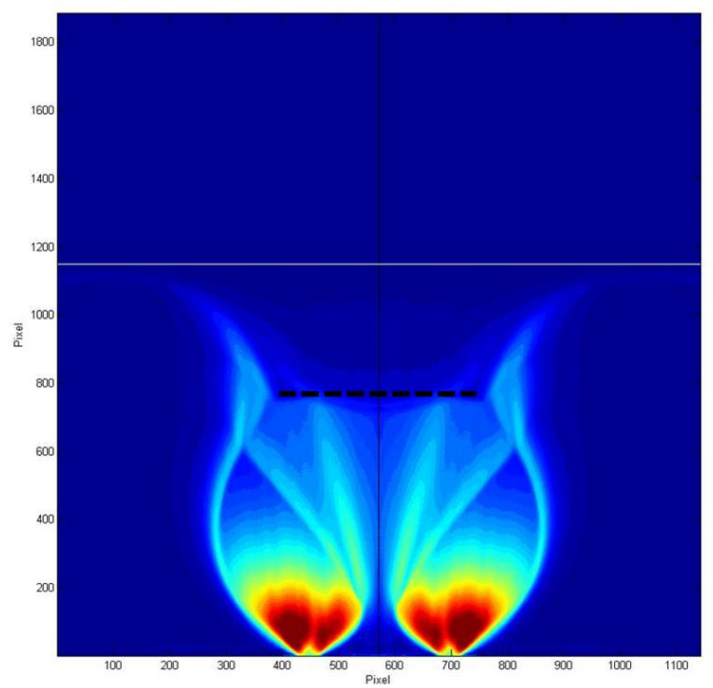

(a) Linear Contour Plot.

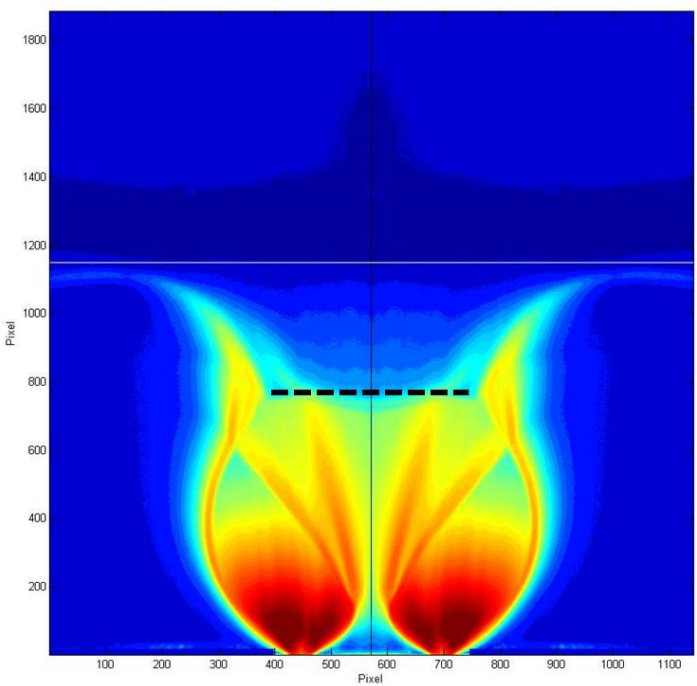

(b) Logarithmic Contour Plot.

Figure 5.4: Disk 1 - Four Jet Free 2.875" Impingement Fluorescence Plots.

The normal shock is marked in the figure with a dashed black line, and occurs at 19.5 $\frac{x}{D}$ downstream of the nozzle exit. The low signal region downstream of the normal shock, including the turning of the flow due to the interaction with the impingement surface, is clear in the logarithmic plot in Figure 5.4 .

A final impingement distance was tested to provide a complete visualization data set for comparison with the two jet experiments. The impingement standoff distance was set to 2.1875 inches. This new standoff distance is equivalent to $22.2 \frac{x}{D}$. The plenum pressure was set to 40 psia, just as in previous experiments. The resultant back pressure was 204.7 millitorr, which corresponds to a $P_{\text {ratio }}$ of 10106. 


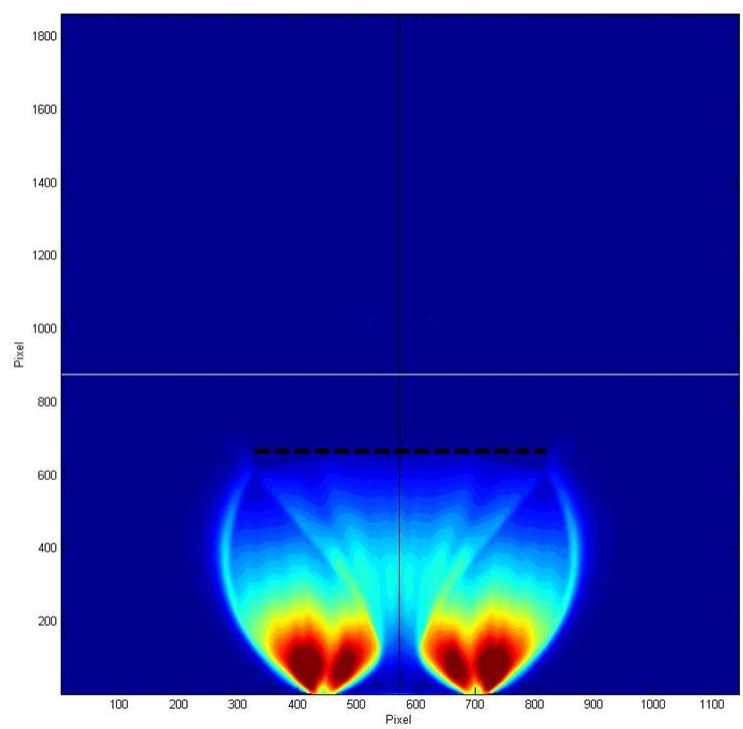

(a) Linear Contour Plot.

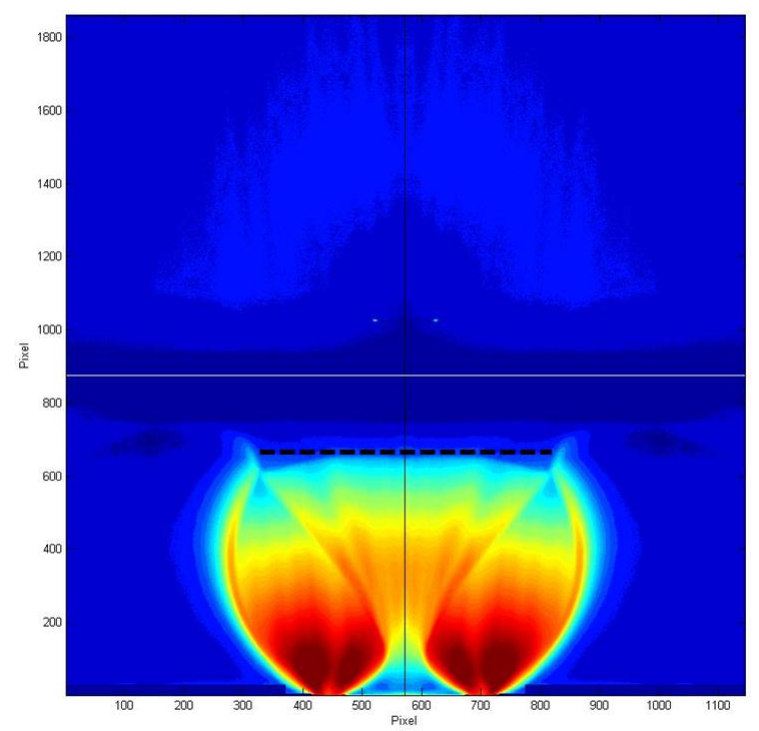

(b) Logarithmic Contour Plot.

Figure 5.5: Disk 1 - Four Jet Free 2.875" Impingement Fluorescence Plots.

Within Figure 5.5, a normal shock feature is located at $16.8 \frac{x}{D}$, and is marked with a black dashed line. The initial interaction with the neighboring jets occurs at 2.9 $\frac{x}{D}$, and results in a maximum flowfield width of $16.0 \frac{x}{D}$. Because the impingement surface is close to the model surface, a significant decrease in fluorescence signal at the impingement surface is noted. Even in the logarithmic plot, there is no signal present at the impingement surface, which is due to the high collisional quenching and post-normal shock static pressure rise in this region.

Since the pressure ratios for each of these experiments do not vary appreciably, the free exhaust and impingement cases are directly comparable for each experimental configuration. Observations about the flowfield features, including jet-jet interactions and jet-jet-surface interactions, can be made by examination of these PLIIF qualitative images. Based on this series of three experiments, the decrease in impingement plate standoff distance yields a smaller $\frac{x}{D}$ distance to the formation of the normal shock. The location of the initial neighboring jet interaction remains essentially the same, as does the maximum width of the flowfield. 
Along with four jet experiments, NASA also expressed interest in imaging a two jet configuration. The two jet configuration is meant to model the interim emergency procedure that NASA Johnson has currently implemented with HTV spacecraft operating within the vicinity of the International Space Station. This interim technique is meant to allow operation in the vicinity of the ISS while mitigating risk until the development of new flight procedures based on the results of this research can be completed.

Testing for the two jet configuration was conducted in a manner identical to the previously presented four jet experiments. The initial two jet experiment showed a significantly different flow field geometry compared to the four jet experiment. Figure 5.6 shows the resulting broadband image. This image has been post-processed using the same procedure applied to the four jet experiments. For the two jet, free exhaust experimental configuration, a plenum pressure of 39.2 psia was utilized. The resulting back pressure was 124.3 millitorr, which translated to a $P_{\text {ratio }}$ of 16310 . In this image, it can clearly be seen that the interaction region between the two jets has a markedly different flow pattern when compared to the four jet interaction. The internal edges of the jet plume impinge closer on the neighboring jet and turn less than in the four jet case. The initial interaction of the neighboring jets occurs at $2.4 \frac{x}{D}$. Also in this image, the external geometry of the jet plume can be seen to form a 'tear drop' shape and exhibit higher downstream flow recombination than in the four jet case. The maximum width of the flowfield is $19.7 \frac{x}{D}$. The presence of a small normal shock along the center of flow, which is very evident in the logarithmic plot of Figure 5.6, occurs at $27.2 \frac{x}{D}$ downstream of the nozzle exit. 


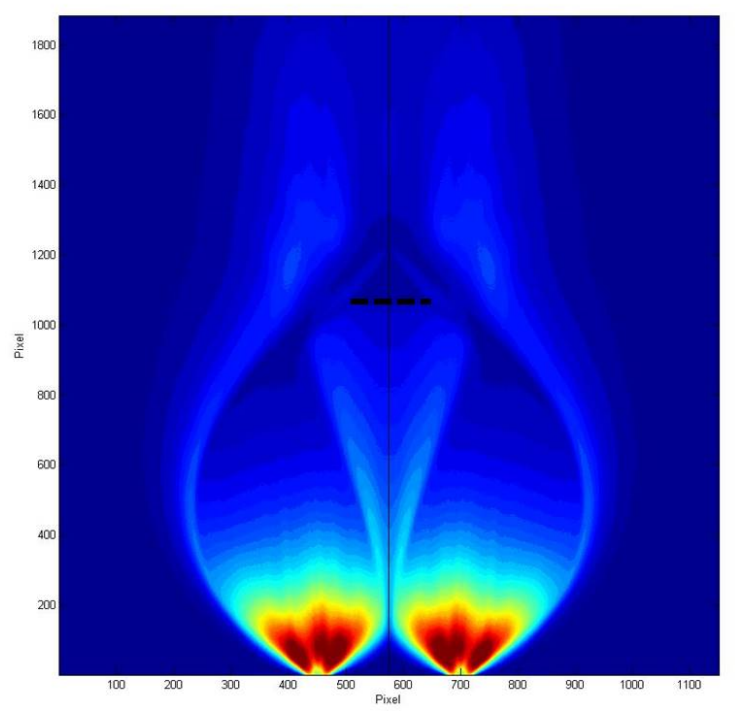

(a) Linear Contour Plot.

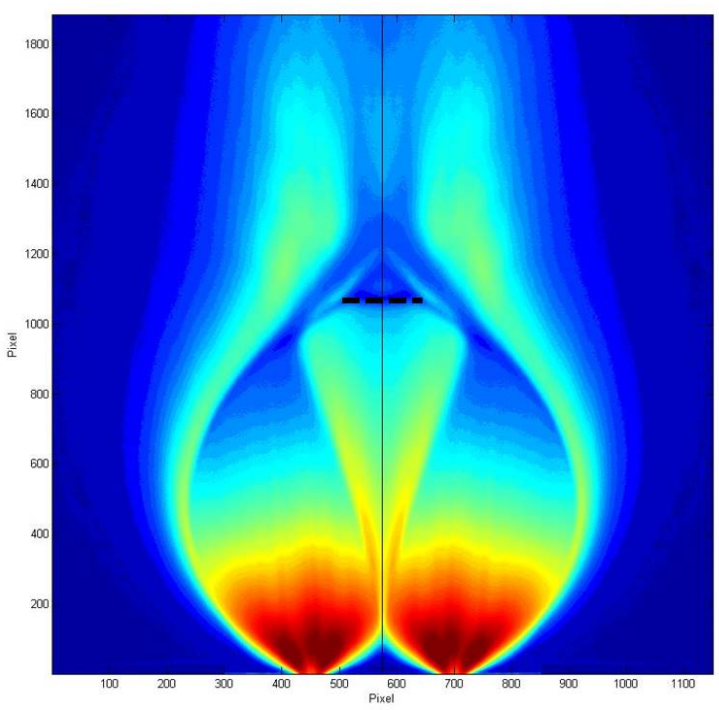

(b) Logarithmic Contour Plot.

Figure 5.6: Disk 1 - Two Jet Free Exhaust Fluorescence Plots.

Due to the two jet configuration currently being utilized in real-world operational spaceflight, a series of two jet impingement experiments was also deemed of interest. First, an experiment was conducted with the impingement standoff distance similar to the four jet investigation. The impingement surface was placed 3.62 inches, or 36.8 $\frac{x}{D}$, with the operating plenum pressure of 40 psia. This resulted in a back pressure of 137.6 millitorr, and a $P_{\text {ratio }}$ of 15033 . The resulting mirrored fluorescence image is shown in Figure 5.7. It is evident from this image that the interaction with the impingement surface, again denoted by a horizontal white line, is minimal. The initial interaction occurs at $2.4 \frac{x}{D}$, and the low density region, shown by the black dashed line, occurs at an $\frac{x}{D}$ of 24.7 downstream of the nozzle exit. The flowfield achieves a maximum width of $18.7 \frac{x}{D}$. 


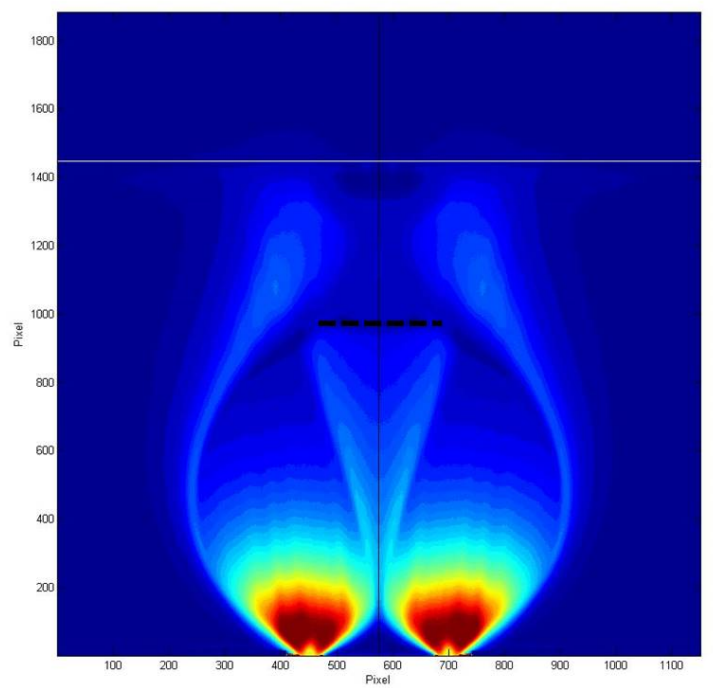

(a) Linear Contour Plot.

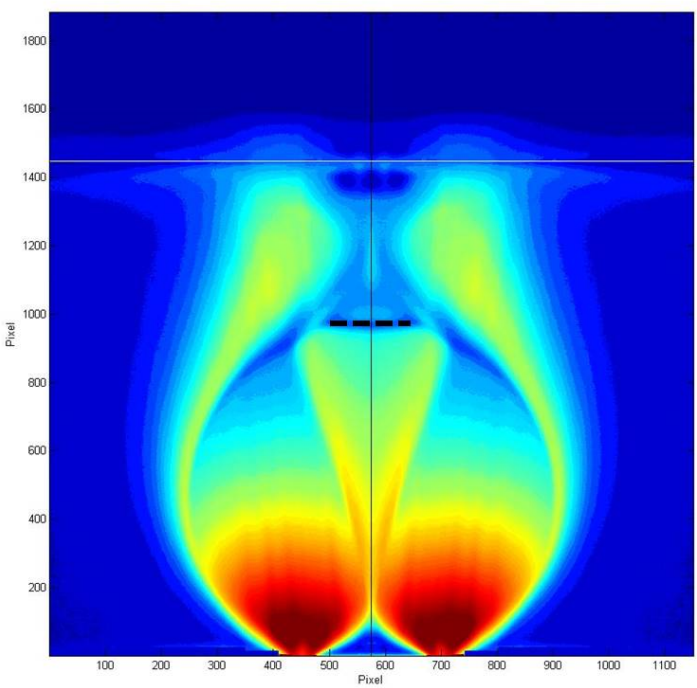

(b) Logarithmic Contour Plot.

Figure 5.7: Disk 1 - Two Jet, 3.62" Impingement Standoff Fluorescence Plots.

In the experimental configuration shown in Figure 5.8, the impingement plate was placed 2.875 inches from the surface of the model. This corresponds to an impingement standoff distance of $29.2 \frac{x}{D}$. The operating plenum pressure was set to 38.5 psia, with a resulting back pressure of 129.1 millitorr. With these operating conditions, the $P_{\text {ratio }}$ is 15422 . The initial interaction of the neighboring jets occurs at $2.0 \frac{x}{D}$. t can clearly be seen in Figure 5.8 that the normal shocks resulting from the interaction with the impingement plate are significantly different than those seen in the four jet impingement flow, or even in the two jet 3.62 inch standoff. As in the four jet impingement experimental image, the normal shocks are denoted by dashed black lines. The most evident difference is the presence of two independent normal shocks. The normal shock over each jet plume occurs at an $\frac{x}{D}$ of 15.5 . This is unique when compared to the four jet experiment, which maintains a single coherent normal shock. The maximum width of the flowfield is $19.4 \frac{x}{D}$. 


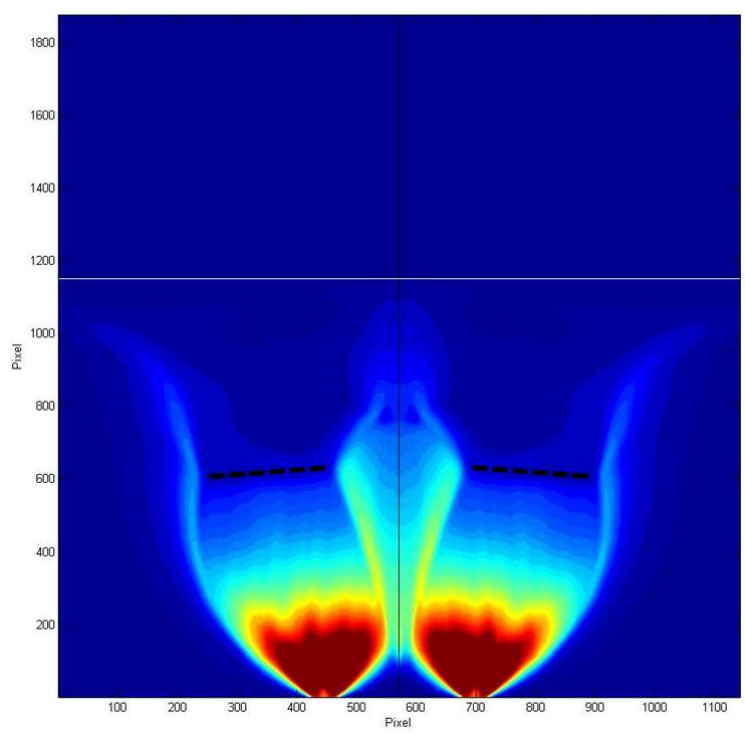

(a) Linear Contour Plot.

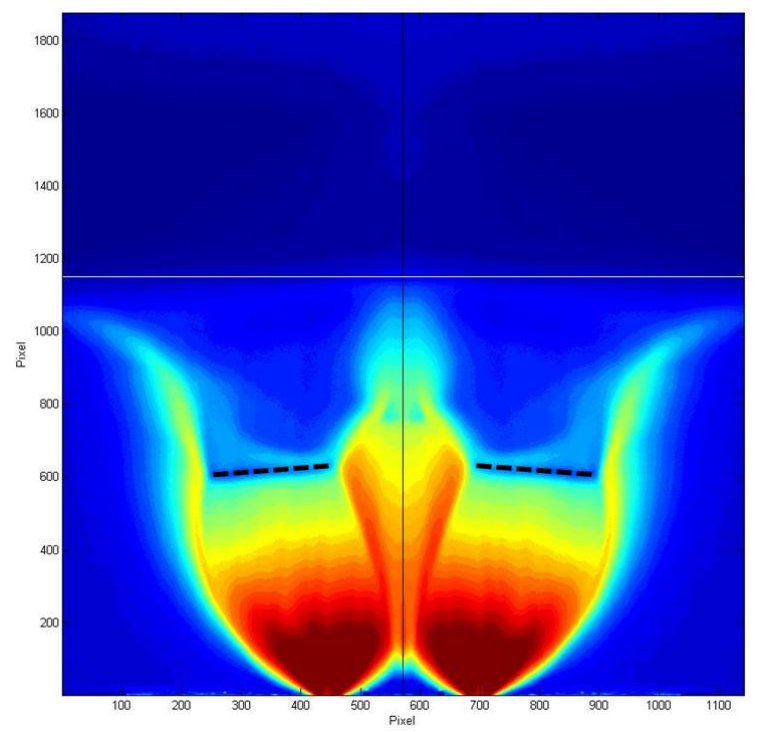

(b) Logarithmic Contour Plot.

Figure 5.8: Disk 1 - Two Jet, 2.875" Impingement Standoff Fluorescence Plots.

A final investigation of the impact of changing the impingement standoff distance was conducted for the two jet flowfield. The final impingement standoff distance was set to 2.1875 inches, or $22.2 \frac{x}{D}$. The plenum pressure for this experiment was set to 40 psia, and the corresponding back pressure was 144.6 millitorr. This yields a $P_{\text {ratio }}$ of 14306. In Figure 5.9, the separate normal shocks continue to be evident. The initial jet interaction occurs at $2.1 \frac{x}{D}$, and the normal shocks occur at $14.1 \frac{x}{D}$. With the closer impingement standoff distance, the normal shocks on each jet plume exhibit a tilting towards the outer region of the flow in Figure 5.9. This results in a wider flowfield, with a maximum width of $20.2 \frac{x}{D}$. The two jet flowfield exhibits unique characteristics as compared to the four jet flow. 


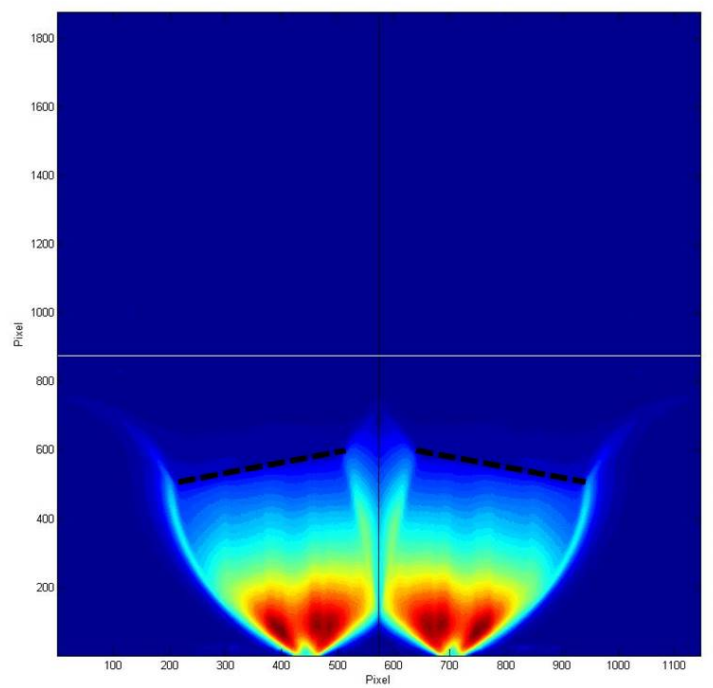

(a) Linear Contour Plot.

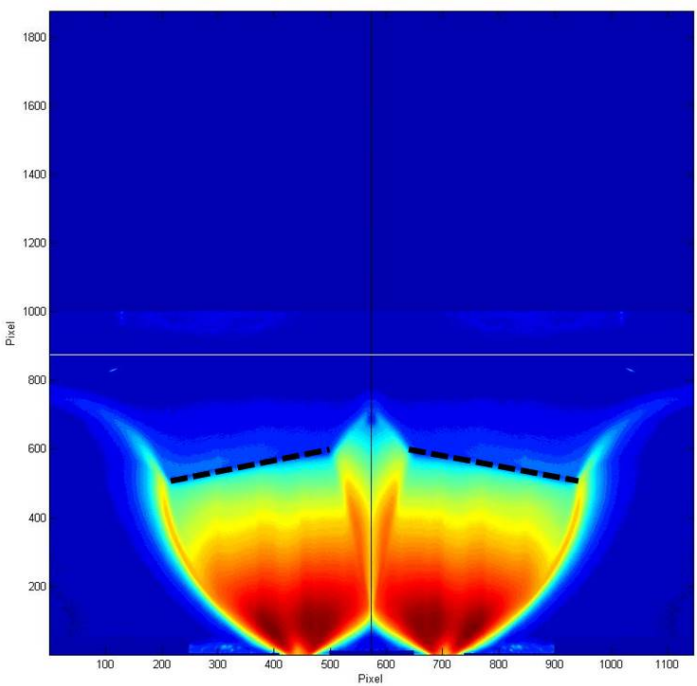

(b) Logarithmic Contour Plot.

Figure 5.9: Disk 1 - Two Jet, 2.1875" Impingement Standoff Fluorescence Plots.

The two jet flow exhibited unique out-of-plane flowfield geometry, as well as the lack of a single normal shock in the interaction region. As the two jet fluid flow interaction increased with the decrease in impingement surface standoff distance, two unique normal shocks formed. Each normal shock remained confined to jet core flow, and exhibit a change in angle as the impingement surface standoff distance decreased. These four jet and two jet experiments provided ample comparison of the effects of the change in impingement standoff distance and number of exhausting jets. In order to completely investigate the neighboring jet interaction, and it's effects on the impingement surface interaction, investigations into the effect of two jet center-tocenter spacing were also conducted and are presented in Section 5.2 and 5.3 .

\subsection{Disk 2 Qualitative Results}

With Disk 1 providing a thorough investigation of the effect of impingement plate standoff distance, as well as the differences in two jet versus four jet flow, experiments utilizing Disk 2 and Disk 3 were also conducted. The use of Disk 2 and 3 
provided a means to investigate the effect of jet center-to-center spacing on the shape of the flowfield and interaction region. The first experiment conducted was a four jet free exhaust experiment using Disk 2 spacing. The center-to-center spacing is 0.874 inches, or $8.9 \frac{x}{D}$. Figure 5.10 below shows minimal neighboring jet interaction. Each jet exhaust shows no neighboring jet interaction throughout the entirety of the flow. Though each jet does not exhibit a distinct normal shock, it does exhibit the characteristic expansion and subsequent compression of the jet flow, as discussed in Chapter 2. The logarithmic plot of this flow does show a very small region of flow existing between each jet plume, but the shape of the jet plume and downstream flow geometry exhibits no signs of interaction.

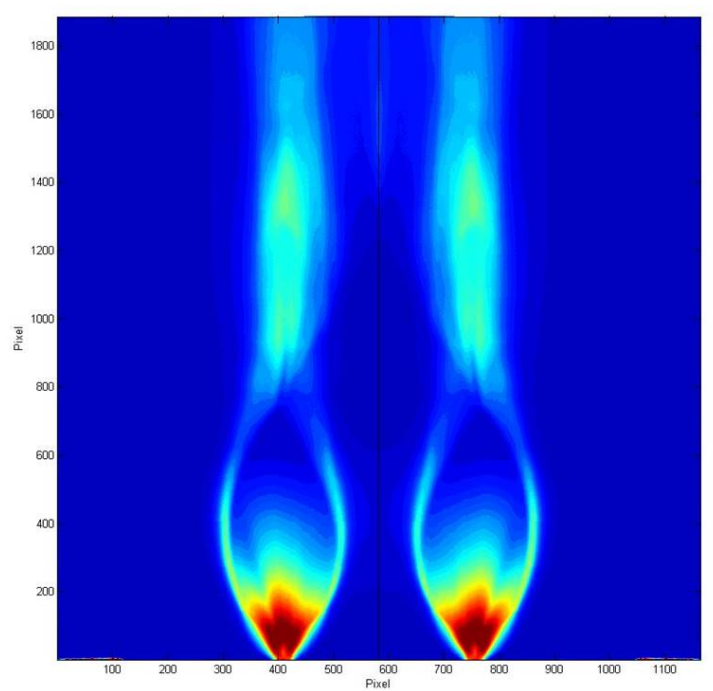

(a) Linear Contour Plot.

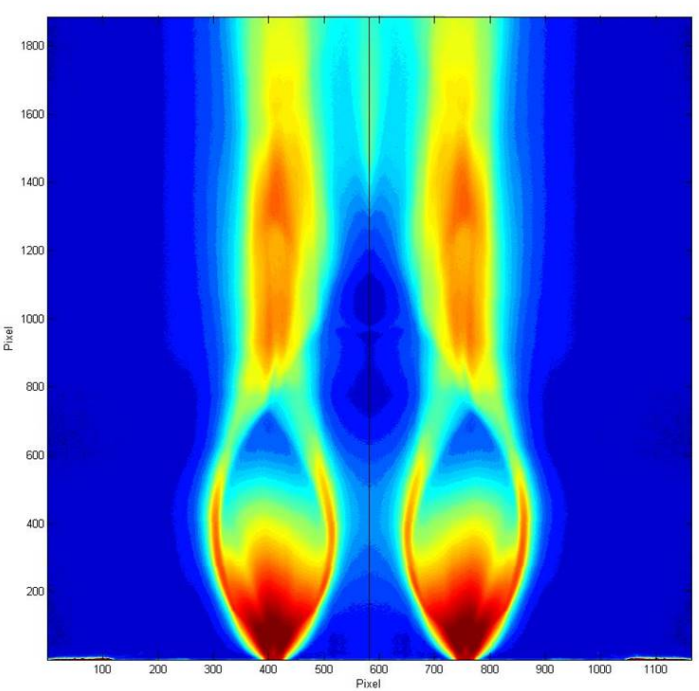

(b) Logarithmic Contour Plot.

Figure 5.10: Disk 2 - Four Jet Free Exhaust.

For the Disk 2 four jet free exhaust experiment, the plenum pressure was set to 40 psia with a back pressure of 225 millitorr. This results in a $P_{\text {ratio }}$ of 9194 . Since there is no significant neighboring jet interactions, the width of the flowfield was measured as the width of a single jet, which is $6.9 \frac{x}{D}$. Without any noticeable interaction in the free exhaust case, an experiment was conducted with a close impingement plate standoff distance. 


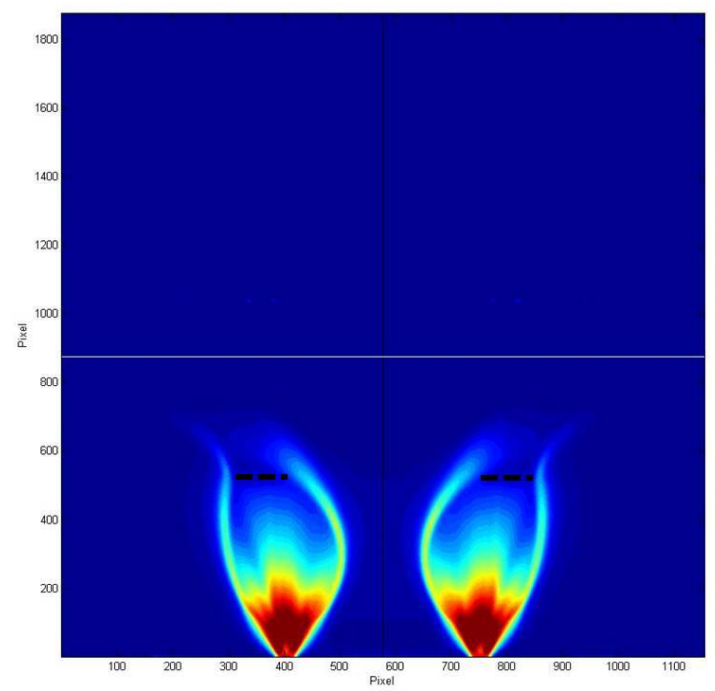

(a) Linear Contour Plot.

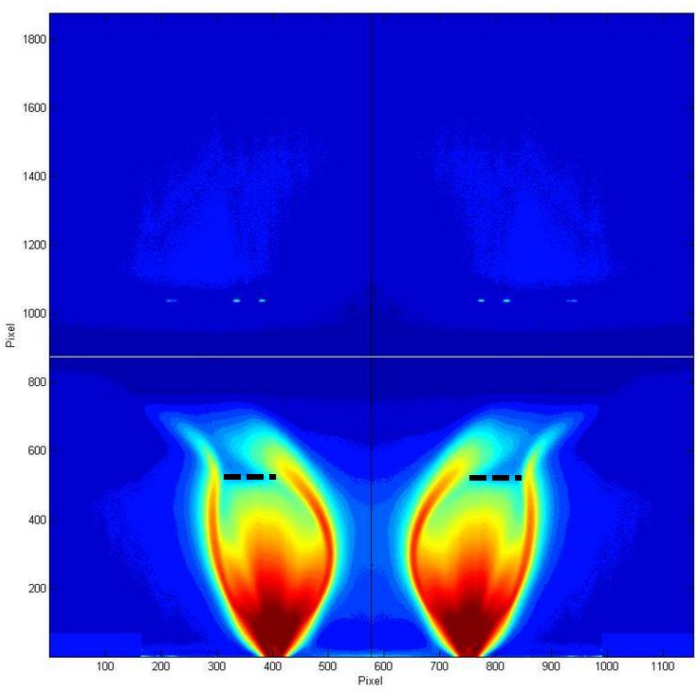

(b) Logarithmic Contour Plot.

Figure 5.11: Disk 2 - Four Jet, 2.1875" Impingement Standoff Fluorescence Plots.

The chosen standoff distance was 2.1875 inches, which is equivalent to an $\frac{x}{D}$ of 22.2. Again, the plenum pressure was set to 40 psia with a back pressure of 216.3 millitorr. This results in a $P_{\text {ratio }}$ of 9564. In Figure 5.11, the formation of a distinct normal shock on each jet plume is present, and occurs at an $\frac{x}{D}$ of 13.2 from the nozzle exit. The maximum width of the jet plume, due to the interaction, is $16.0 \frac{x}{D}$. From these images the turning of the jet plume is evident, as is an asymmetry in the jet plume that indicates a neighboring jet interaction. While the individual jet plumes do not interact directly, the flow is turned due to the location of the constant pressure boundary produced by the compressive interaction with the impingement surface. This compressive interaction results in the unique curvature of the inner boundaries of the jet flow, where the inner boundaries are the ones nearest to the disk centerline. The interaction location is not identifiable in these images, based on using the $15 \%$ of maximum fluorescence criteria applied to all other experimental images, because the fluorescence signal is so low in the region of interaction. The outer flow boundaries on each jet maintains a typical jet boundary shape. 


\subsection{Disk 3 Qualitative Results}

Even though minimal interaction was observed in the qualitative broadband images taken for the Disk 2 nozzle center-to-center spacing, an investigation utilizing Disk 3 is necessary to provide a complete investigation of the neighboring jet interactions. Disk 3 has a center-to-center spacing of 2.248 inches, or $22.8 \frac{x}{D}$. The experimental configurations were similar to the experiments for Disk 2, and is composed of a four jet free exhaust experiment and a close impingement standoff distance experiment.

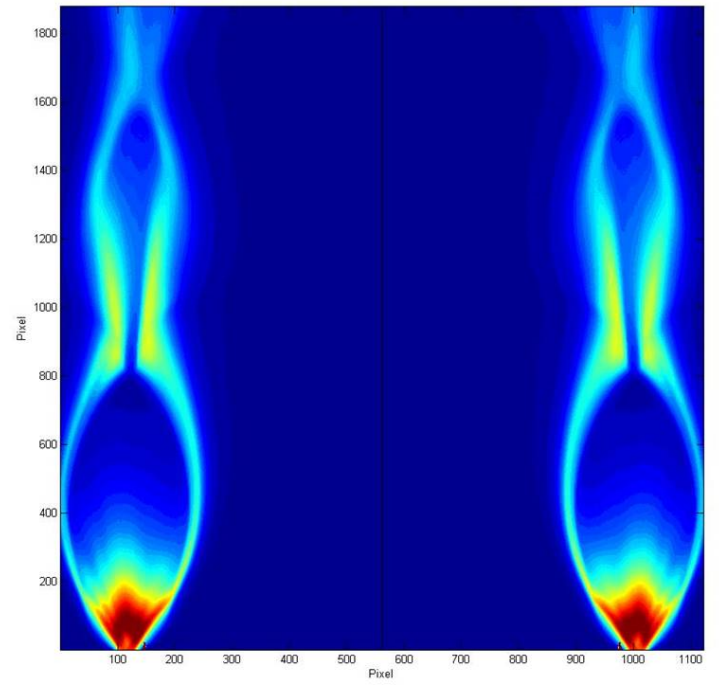

(a) Linear Contour Plot.

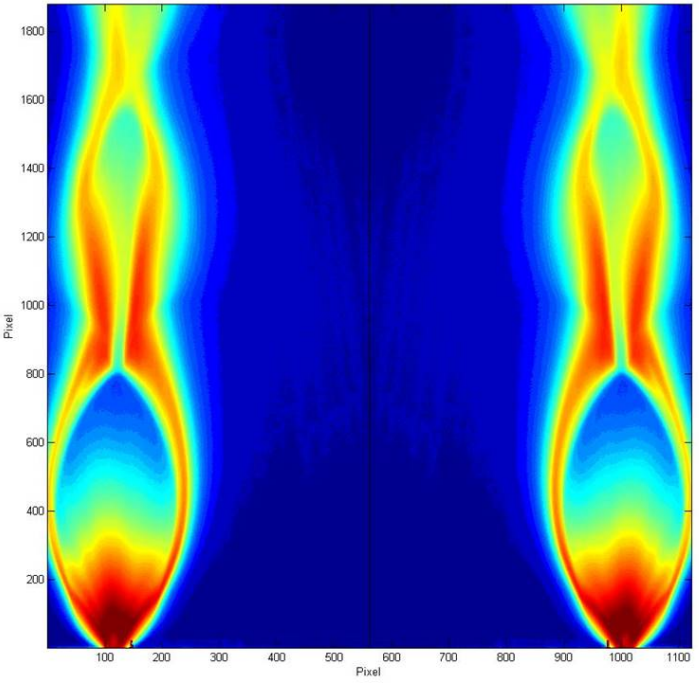

(b) Logarithmic Contour Plot.

Figure 5.12: Disk 3 - Four Jet Free Exhaust Fluorescence Plots.

Figure 5.12 contains the results of the four jet free exhaust experiment using Disk 3. The plenum pressure for this experiment was set to 40 psia and achieved a back pressure of 234.8 millitorr. These conditions results in a $P_{\text {ratio }}$ of 8810 . As expected, there is no neighboring jet interactions present with this large center-to-center spacing. With no interaction present in this experiment, the maximum single jet width was measured to be $7.3 \frac{x}{D}$. Again, there is no distinct normal shock present in the individual jet plumes, but the characteristic compression and expansion of the flow 
as it proceeds downstream is observed.

In order to further characterize the impingement surface interaction as the neighboring jet center-to-center distance increased, and experiment was conducted with a close impingement standoff distance. An impingement standoff distance of 2.1875 inches, $22.2 \frac{x}{D}$, was selected to match the Disk 2 impingement experiment.

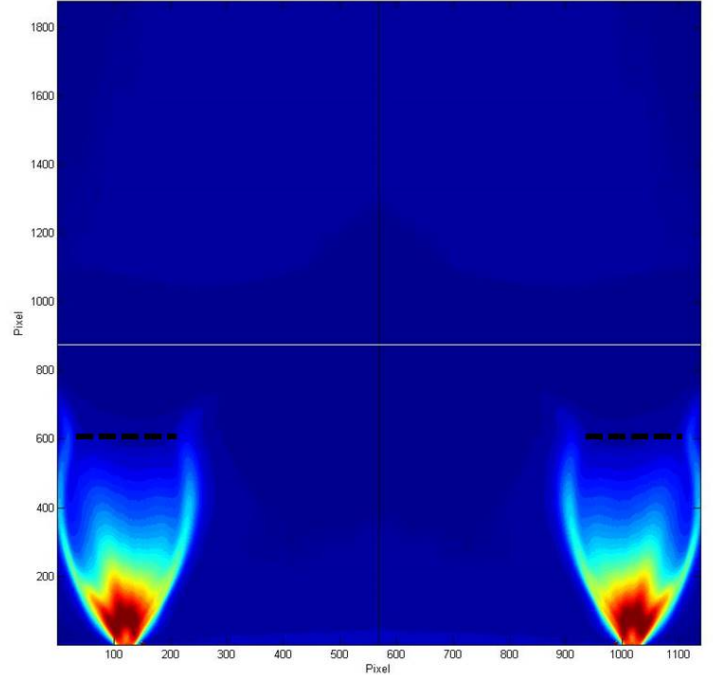

(a) Linear Contour Plot.

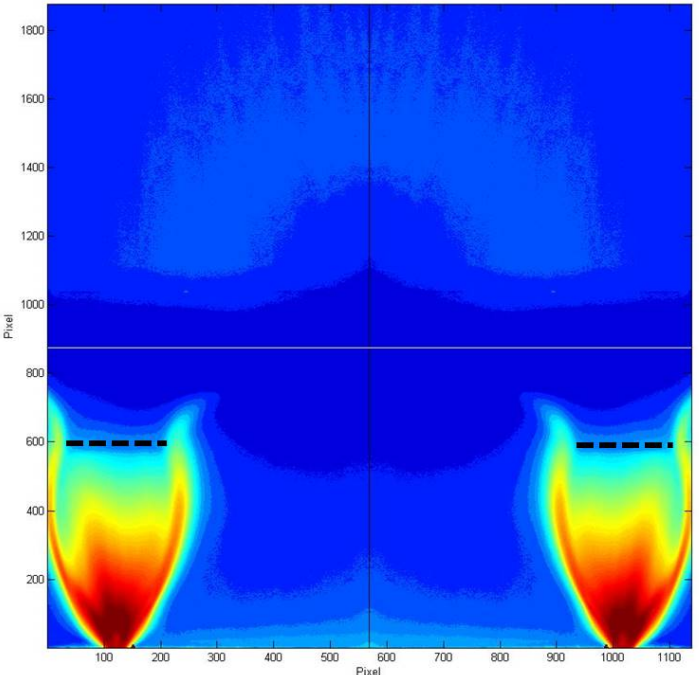

(b) Logarithmic Contour Plot.

Figure 5.13: Disk 3 - Four Jet, 2.1875" Impingement Standoff Fluorescence Plots.

As with the Disk 2 impingement experiment, the plenum pressure was set to 40 psia, which yielded a back pressure of 227.9 millitorr. This results in a $P_{\text {ratio }}$ of 9077. In Figure 5.13, the formation of a distinct normal shock can clearly be seen, with a downstream location of $14.4 \frac{x}{D}$. Also present in this image is no evidence of neighboring jet interactions at the impingement surface. This is very clear as the jet boundaries maintain their symmetric shape. The maximum width of a single jet was measured to be $7.2 \frac{x}{D}$. The normal shock occurs farther downstream than the Disk 2 impingement experiment, and the maximum jet width is also smaller. The smaller jet width is attributable to the impingement surface neighboring jet interaction, as is the farther upstream location of the normal shock. In the following section, side-by-side 
comparisons of the previously discussed flowfields will be presented, along with tables compiled to provide a summary of the geometric measurements collected from the qualitative broadband images included in this chapter.

\section{$5.4 \quad$ Flowfield Comparisons}

In this section, visual comparisons of flowfields will be presented, along with quantitative flowfield geometry measurements. Using the visualization images presented in the previous section, measurements of the initial neighboring jet interaction location, maximum flowfield width, and location of shock structures can be measured. As mentioned earlier in this chapter, a criteria of $15 \%$ fluorescence signal is used to determine the jet boundaries and normal shock location within the broadband visualization images. The first comparison presented is between the four jet free exhaust flow cases for each of the nozzle disks. This comparison will identify the effect of neighboring jet spacing on flow interaction.

Figure 5.14 contains the Disks 1,2 and 3 free exhaust linear fluorescence visualization data. The $P_{\text {ratio }}$ for each experiment is 8663,9194 , and 8810 respectively. The significant neighboring jet interaction for Disk 1 is clear, while no interaction is observed for either Disks 2 or 3. The large core flow deflection from the jet-jet interaction is also evident in the Disk 1 image, whereas the jet plumes for Disks 2 and 3 show no interaction and are very similar in size and shape. From these images, the interaction in Disk 1 is observed at $2.7 \frac{x}{D}$ and a maximum flowfield width is measured to be $16.3 \frac{x}{D}$. Also observed in the Disk 1 visualization is the presence of the normal shock at $26.8 \frac{x}{D}$, where Disks 2 and 3 exhibits no normal shock formation. 


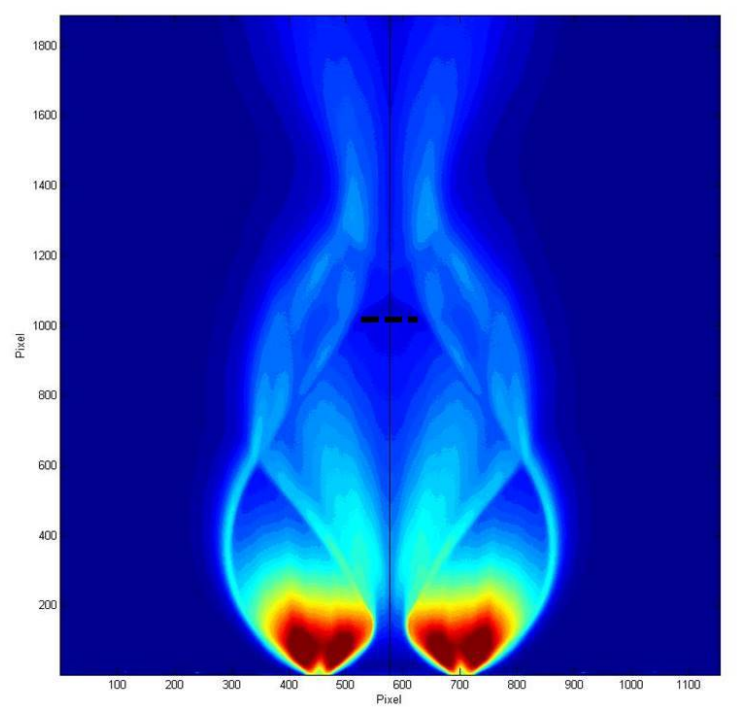

(a) Disk 1.

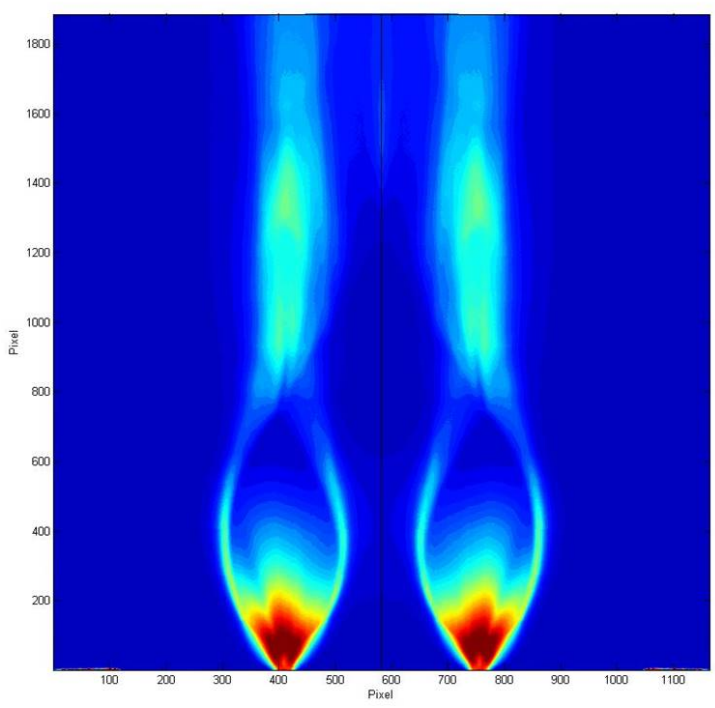

(b) Disk 2 .

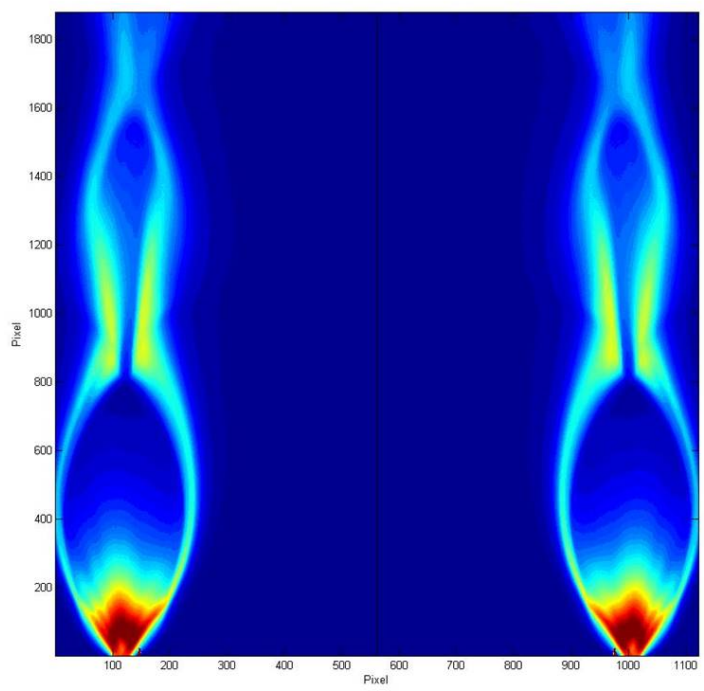

(c) Disk 3 .

Figure 5.14: Comparison of Four Jet Free Exhaust Fluorescence Visualizations.

It is evident from these images that neighboring jet interaction is highly dependent on jet center-to-center spacing, as expected. Even with Disk 1 corresponding to a geometric scaling factor 1.6 times greater than HTV-3, a significant amount of neighboring jet interaction is occurring. Table 5.1 contains a summary of the flow measurements taken from the images in Figure 5.14 


\begin{tabular}{|c|c|c|c|}
\hline Experimental Configuration & Interaction Location (downstream) & Maximum Flowfield Width & Shock Location (downstream) \\
\hline \hline Disk $1-4$ jet, free exhaust & 2.7 & 16.3 & 26.8 \\
\hline Disk $2-4$ jet, free exhaust & NONE & $6.9^{*}$ & NO SHOCK \\
\hline Disk $3-4$ jet, free exhaust & NONE & $7.3^{*}$ & NO SHOCK \\
\hline
\end{tabular}

Table 5.1: Pertinent geometry measurements, in $\frac{x}{D}$.

Along the same line as the free exhaust comparison, a comparison between impingement experiment cases reinforces the center-to-center spacing impact on neighboring jet interactions. For this comparison, Disks 1, 2 and 3 are presented with identical impingement standoff distances. The $P_{\text {ratio }}$ for each disk is 10106, 9564, and 9077 respectively. Selecting an impingement standoff distance of 2.1875 inches, the effect of jet center spacing can be clearly observed in Figure 5.15. While all three interaction cases exhibit the formation of a normal shock, Disk 1 and Disk 2 exhibit unique neighboring jet interactions. Disk 1 continues to exhibit the large neighboring jet interaction, and resultant core flow deflection. Interestingly, Disk 2 also exhibits core flow deflection resulting from the proximity of the free pressure boundary created by the compressive interaction with the impingement surface. Disk 3, on the other hand, exhibits no interaction and each jet plume forms a normal shock while maintaining flow symmetry on each jet plume. It is interesting to note that the downstream location of the normal shock for Disk 1 is $16.8 \frac{x}{D}$, whereas for Disk 2 the normal shock is located at $13.2 \frac{x}{D}$. With no neighboring jet interaction, Disk 3 formed normal shocks at $14.4 \frac{x}{D}$ downstream of the nozzle exit. This indicates that the interaction of the neighboring jet for Disk 1 causes the formation of the normal shock to occur further downstream. The interacting flowfield from Disk 1 and 2 also shared an identical maximum width of $16.0 \frac{x}{D}$, whereas the flowfield maximum for Disk 3 was only measured for single jet, since there was no interaction. The single jet width for Disk 3 is $7.2 \frac{x}{D}$. 


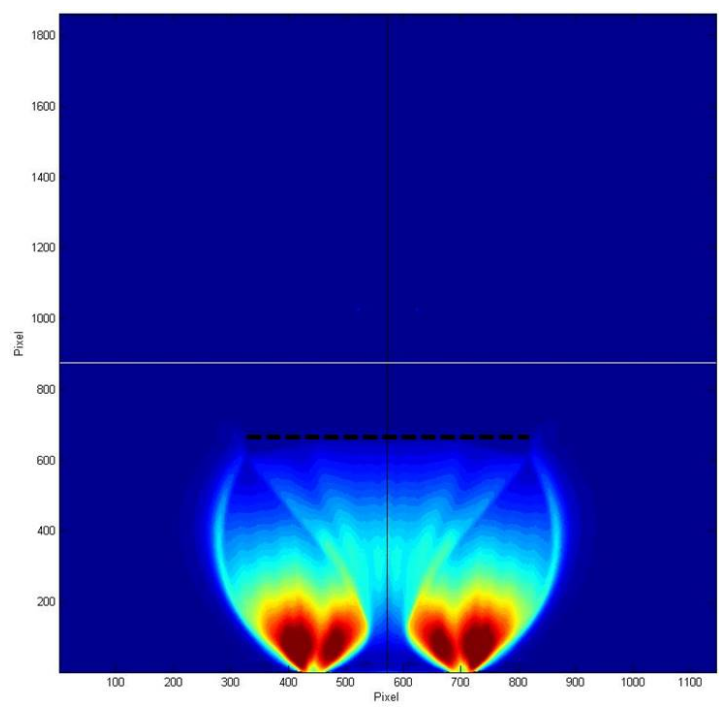

(a) Disk 1.

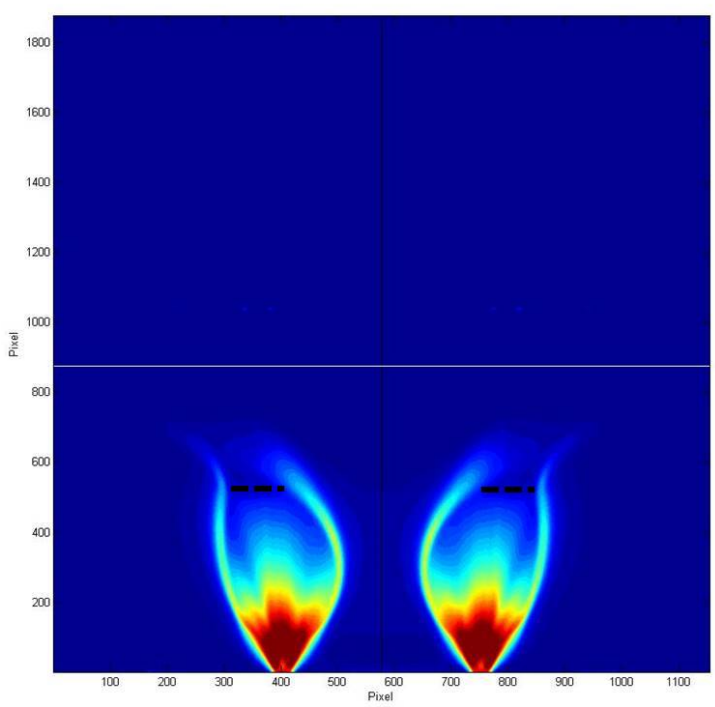

(b) Disk 2 .

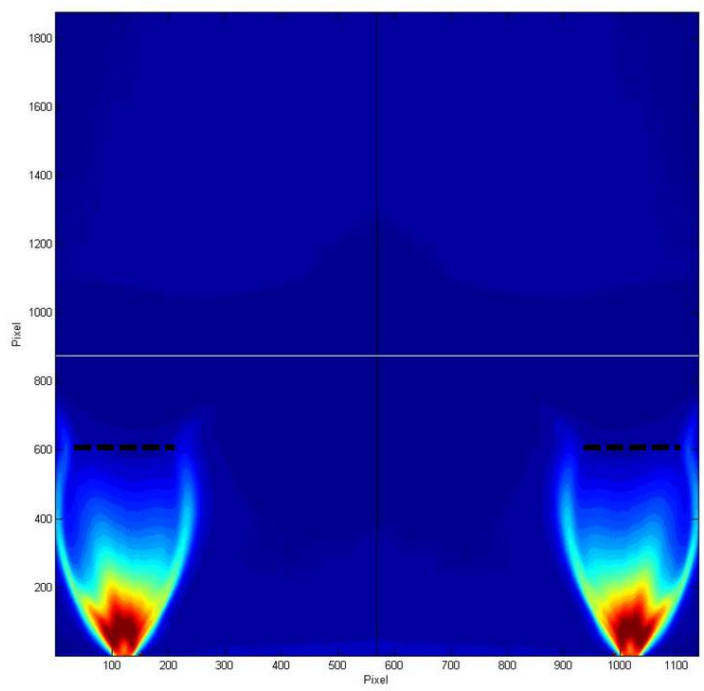

(c) Disk 3 .

Figure 5.15: Comparison of Four Jet Free 2.1875" Standoff Visualizations.

Table 5.2 contains a summary of flowfield geometry measured from the data represented in Figure 5.15 .

\begin{tabular}{|l|c|c|c|}
\hline Experimental Configuration & Interaction Location (downstream) & Maximum Flowfield Width & Shock Location (downstream) \\
\hline \hline Disk 1 - 4 jet, 2.1875" standoff & 2.9 & 16.0 & 16.8 \\
\hline Disk $2-4$ jet, 2.1875" standoff & UNIDENTIFIED & 16.0 & 13.2 \\
\hline Disk 3 - 4 jet, 2.1875" standoff & NONE & $7.2^{*}$ & 14.4 \\
\hline
\end{tabular}

Table 5.2: Pertinent geometry measurements, in $\frac{x}{D}$. 
Finally, a comparison between two and four jet experiments will show the effect of the out-of-plane jets in the free jet case on neighboring jet interactions, as well as the effect on impingement surface interactions. For this comparison visualization images from Disk 1 are presented. The impingement experiment comparison uses the 2.875 inch standoff distance configuration. The $P_{\text {ratio }}$ for the four jet free exhaust experiment is 8663 , and for the two jet free exhaust the $P_{\text {ratio }}$ is 16310 . For the four jet impingement, the $P_{\text {ratio }}$ is 9808 , while the two jet impingement $P_{\text {ratio }}$ is 15422 . A direct comparison of the flowfield width and normal shock location between the four and two jet configuration is difficult due to the large difference in pressure ratio. The large difference in pressure ratio can account for differences in shock location and flow width, but for the purpose of a visual comparison the results demonstrate the marked differences in the flow structure.

From Figure 5.16, the larger interaction region present between the neighboring jets in the four jet free exhaust experiment is clear. The presence of the larger core flow deflection in the four jet case is also clear. Similar qualitative assertions can be made for the impingement experiments. The unique formation of single versus dual normal shocks is clear in Figure 5.16. The significant neighboring jet interaction remains present in the four jet impingement results, as well. It becomes clear from the side-by-side comparison of these two jet flow configurations that the out-of-plane jets in the four jet experiments provide significant influence on the interaction region, as well as the deflection of the jet core fluid flow and formation of a normal shock. From these comparisons, it appears that the two out-of-plane jets present in the four jet flow configuration contribute to a larger mixing interaction region along the flow centerline. Without the two out-of-plane jets contributing to the flow interaction, the two jet flow configuration appear to exhibit less mixing interaction, as well as less jet core fluid deflection. It is not possible to draw direct comparisons between the two and four jet flow with regards to maximum flowfield width due to the significant 
difference in pressure ratio between the flow configurations. The introduction of an impingement surface also appears to contribute to the interaction of neighboring jets, as evidenced by the observed interaction in the Disk 2 impingement experiment.

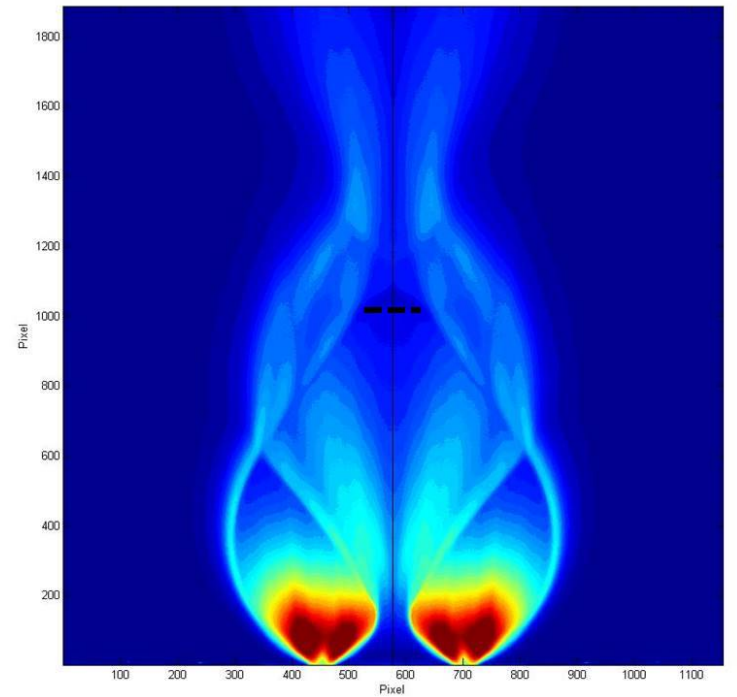

(a) Disk 1, 4 Jet Free Exhaust.

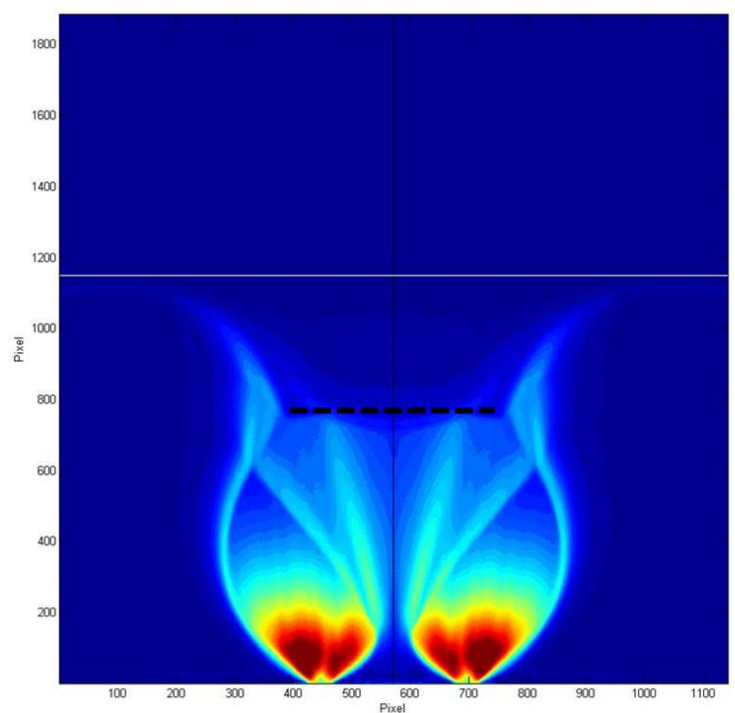

(c) Disk 1, 4 Jet 2.875" Standoff.

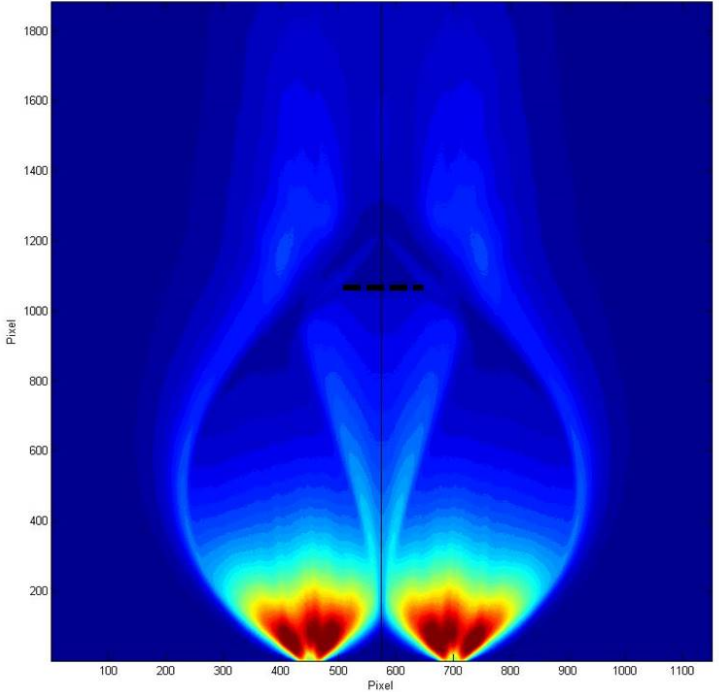

(b) Disk 1, 2 Jet Free Exhaust.

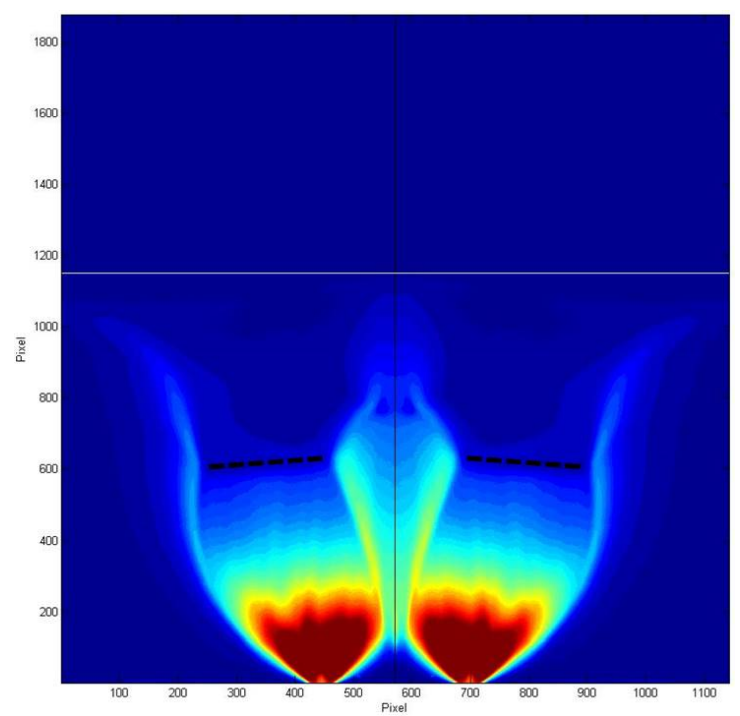

(d) Disk 1, 2 Jet 2.875" Standoff.

Figure 5.16: Comparison of Disk 1 Four and Two Jet Fluorescence Visualizations.

Though quantitative comparisons cannot be made directly between two and four jet experiments, the flow measurements obtained from the data in Figure 5.16 are 
presented in Table 5.3 .

\begin{tabular}{|c|c|c|c|}
\hline Experimental Configuration & Interaction Location (downstream) & Maximum Flowfield Width & Shock or Low Density Location (downstream) \\
\hline \hline Disk 1 - 4 jet, free exhaust & 2.7 & 16.3 & 26.8 \\
\hline Disk 1 - 2 jet, free exhaust & 2.4 & 19.7 & 27.2 \\
\hline Disk 1 - 4 jet, 2.875" standoff & 2.9 & 16.4 & 19.5 \\
\hline Disk 1 - 2 jet, 2.875" standoff & 2.0 & 19.4 & 15.5 \\
\hline
\end{tabular}

Table 5.3: Pertinent geometry measurements, in $\frac{x}{D}$.

From the visualization data presented in this chapter, a unique picture of the complex nature of the neighboring jet interactions begins to take shape. The impact of the out-of-plane jets on the interacting region is seen to be significant. This impact also manifests in the impingement experiments, where unique differences in the formation of normal shocks within the flow further add to the variation in flow geometry that is dependent on the number of exhausting jets. In order to increase the understanding of the complex nature of the flow interactions, a quantitative measure of density ratio, as well as a quantification of the mixing area, is presented in Chapter 6.

\subsection{PLIIF Fluorescence Phenomenon}

In this section, a spectroscopic phenomena associated with iodine fluorescence in hypersonic flowfields will be discussed in depth. As discussed in Chapter 3, when the flowfield is at very low pressure and temperature, the iodine fluorescence spectrum resolves into hyperfine components, as seen in Figure 3.5. An example of this is seen in Figure 5.17 . 


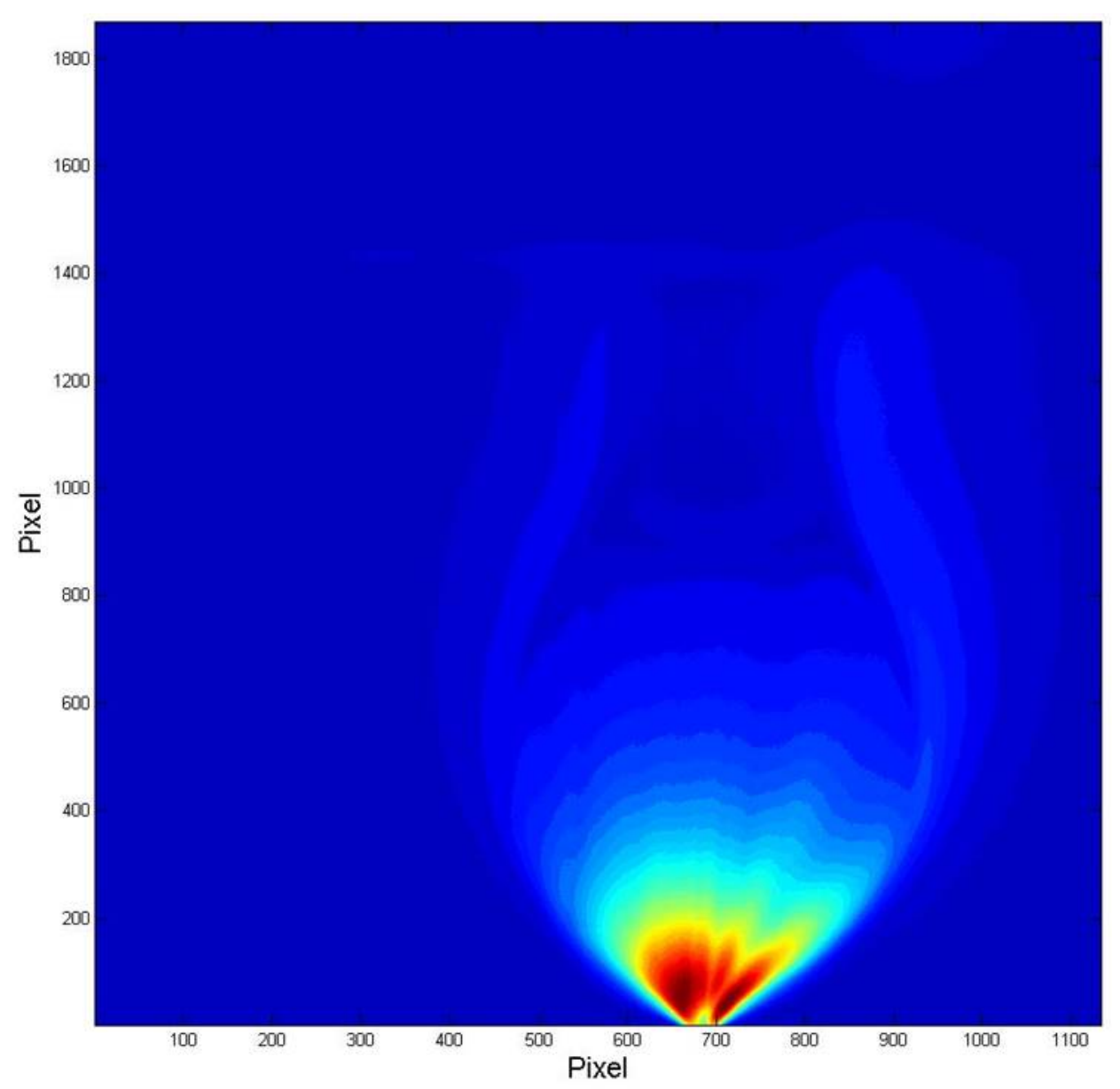

Figure 5.17: Fluorescence Contour Plot for a Single Jet, Free Exhaust.

It has been observed in previous experiments ${ }^{49151150}$, that within the core structure of a supersonic, under-expanded jet the hyperfine spectrum can be resolved in the fluorescence signal. In experiments associated with the main focus of this research, the resolution of the hyperfine spectrum in supersonic jet core fluorescence signals was investigated. The rise of the hyperfine spectrum in the jet core fluorescence signal occurs because of the spatial spread of the velocity vector directions, and Doppler shift, in the jet core. This is very similar to what occurs when the narrowband laser technique is used, as outlined in Chapter 3. The spectra discussed in this section corresponds to tuning a narrowband laser across the P13 / R15 absorption lines. These lines correspond to the absorption spectrum. With the broadband PLIIF technique, the fluorescence emissions from all excited states are collected without 
resolving the spectra. It will be shown in this section that the absorption spectra are generated within an expanded jet fluorescence by the distribution of velocities within the jet core flow, thereby providing a spatial resolution equivalent to that seen in Figure 5.19 .

As detailed in Chapter 4, during normal experimental procedures the camera exposure time is established in order to saturate the jet core, and in turn provide the optimal downstream fluorescence signal in the rarefied regions of flow. For this investigation, the camera exposure time was set in order to allow the hyperfine spectrum to resolve in the core fluorescence signal. In order to verify that the fluorescence signal does indeed reflect the iodine hyperfine spectrum, an established iodine fluorescence model ${ }^{49151}$ is utilized. Three outputs from the iodine model is shown in Figure 5.18 , where the bottom plot demonstrates the resolution of the hyperfine peaks in the iodine spectrum at low temperature and pressure. The top spectrum is representative of flow conditions corresponding to 100 Kelvin and $3.34 \times 10^{-2}$ atmosphere, which corresponds to a Mach number of approximately 3.2 for a $T_{0}$ of $300 \mathrm{~K}$, and a $P_{0}$ of 23 psia. The middle spectrum corresponds to conditions at 30 Kelvin and $5 \times 10^{-4}$ atmosphere, with a Mach number of approximately 6.6 for the same $T_{0}$ and $P_{0}$. The bottom spectrum, which corresponds to 10 Kelvin and $1.32 \times 10^{-5}$ atmosphere, as well as a Mach number of approximately 11.9 for the same $T_{0}$ and $P_{0}$ as the previous spectra. This final spectrum represents conditions that are found within the core of the under-expanded jet used in this research. 

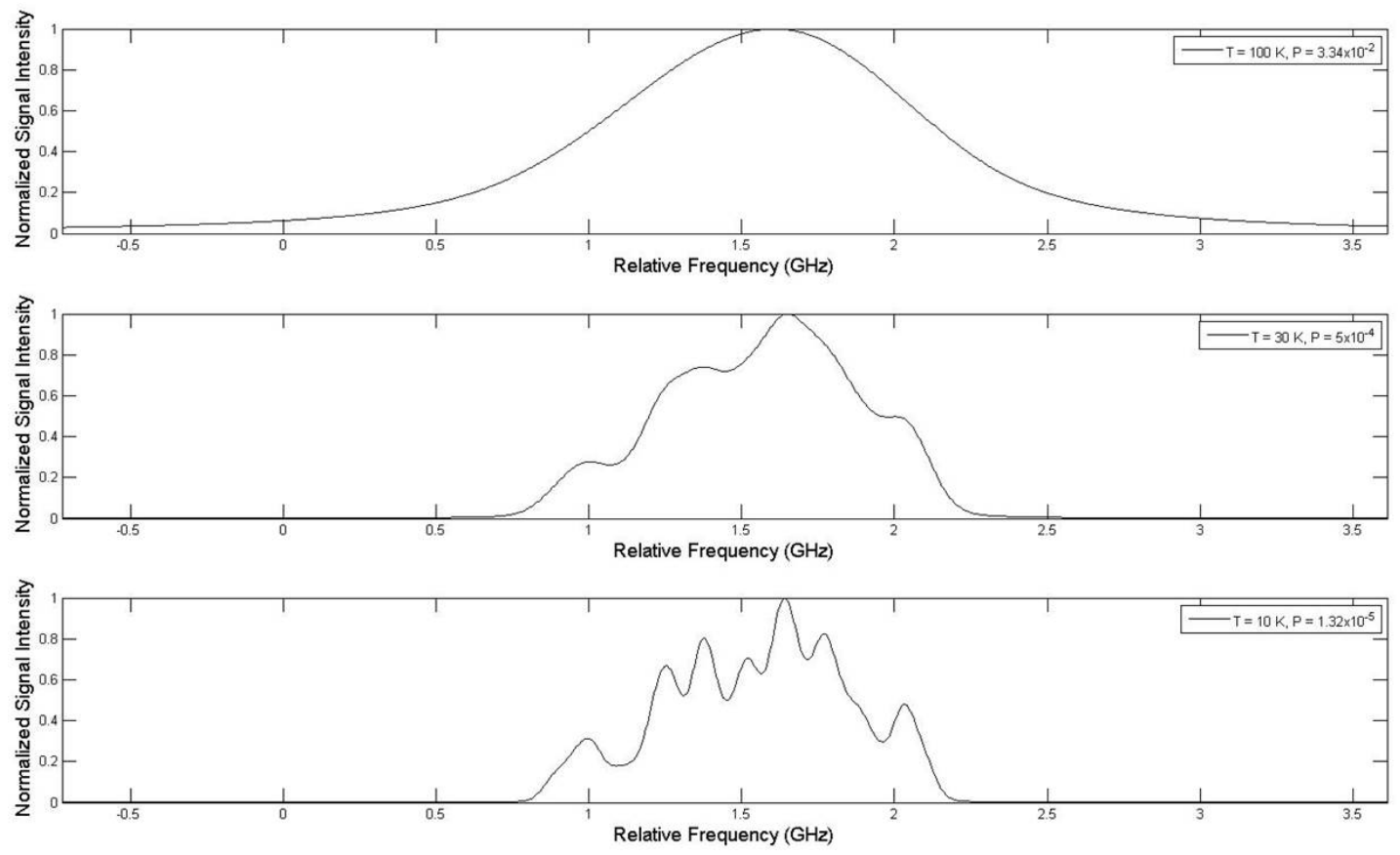

Figure 5.18: Predicted Absorption Spectrum Output from the Iodine Model.

In order to use the iodine model to demonstrate the resolved spectra in the underexpanded jet, the velocity and thermodynamic conditions are needed at discrete flowfield locations. The phenomena is confined to the jet core, therefore the isentropic chain is used to calculate the pressure and temperature at discrete locations in the jet core with predicted Mach numbers. Direct quantitative velocity measurements were not in the scope of this research, therefore a numerical prediction method for the flowfield velocity is needed. Since finite element or finite volume numerical techniques were not available to predict flowfield conditions, an assumption of uniform conditions perpendicular to the jet centerline was made. With this assumption, only predictions of Mach number are needed to calculate velocity and thermodynamic conditions along the centerline. The calculated conditions were assumed to be constant through the normal to the centerline point within the jet core. In 1965, Ashkenas and Sherman published an empirical equation that predicts the centerline Mach number variation in the inviscid central core of a supersonic free jet $\frac{60}{6}$. The empirical equation, 
based on the method of characteristics, is presented as:

$$
M=A\left(\frac{x-x_{0}}{D}\right)^{\gamma-1}-\frac{1}{2} \frac{\left(\frac{\gamma+1}{\gamma-1}\right)}{A\left(\frac{x-x_{0}}{D}\right)^{\gamma-1}}
$$

where $\gamma$ is 1.4 for a diatomic gas and the fitting constants $A$ and $\frac{x_{0}}{D}$ are 3.65 and 0.40 , respectively. Note that this equation is for a jet with sonic exit conditions. The initial value for $\frac{x_{0}}{D}$ was determined by projecting the characteristic solution streamlines back to the jet centerline, which gives the 'source' from which the streamlines radiate. For this given value, Equation 5.2 is re-arranged to solve for A. When values for the Mach number and $\frac{x}{D}$ are substituted into the characteristic solution, a valid value of $x_{0}$ results in a constant value of $A$, which indicates the threshold of the inertiadominated region 60 . Using Equation 5.2, along with the isentropic chain relating pressure, temperature, and density to Mach number:

$$
\begin{aligned}
& \frac{p}{p_{t}}=\left(1+\frac{\gamma-1}{2} M^{2}\right)^{-\frac{\gamma}{\gamma-1}} \\
& \frac{T}{T_{t}}=\left(1+\frac{\gamma-1}{2} M^{2}\right)^{-1} \\
& \frac{\rho}{\rho_{t}}=\left(1+\frac{\gamma-1}{2} M^{2}\right)^{-\frac{1}{\gamma-1}}
\end{aligned}
$$

allows for the solution of Equation 3.18 to predict the fluorescence signal along the single sonic jet centerline. The prediction is shown in Figure 5.19, where the solution of Equation 5.2 is shown in purple and marked in the legend as 'Un-adjusted A\&S Solution'. It is clear that the initial solution to Equation 5.2 does not produce a reasonable prediction of centerline fluorescence. According to Ashkenas and Sherman, the fitting constants $A$ and $\frac{x_{0}}{D}$ were determined by matching the solution of Equation 5.2 to experimental data. In order to refine the prediction of Mach number for the research presented here, the fitting constants were numerically adjusted in order to provide a more accurate comparison with the Mach 5 single jet free exhaust experimental data collected in this research. It was found that a value for $A$ of 3 , and $\frac{x_{0}}{D}$ 
of 2 provided the best correlation to the experimental data with a Mach 5 exit condition. With the adjusted solution to Equation 5.2, the results of Equations 5.3 can be used to solve the iodine fluorescence model. The new solution to Equation 3.18 is shown in Figure 5.19 as the light blue line and labeled in the legend as 'Adjusted A\&S Solution', which now agrees well with experimental centerline data. It is worth noting that the original values for $A$ and $\frac{x_{0}}{D}$ from the Ashkenas and Sherman fit were for a sonic orifice jet plume, whereas the flow studied in this research exits at Mach 5 from a contoured nozzle. Because of this fact, it is reasonable that the value for $A$ and $\frac{x_{0}}{D}$, characterizing the 'source' of the flow at the jet exit, should be larger than those originally presented for a sonic exit jet by Ashkenas and Sherman. Given the Mach distribution and the assumption of constant properties normal to the centerline, velocity, pressure and temperature can be calculated along spatial contours.

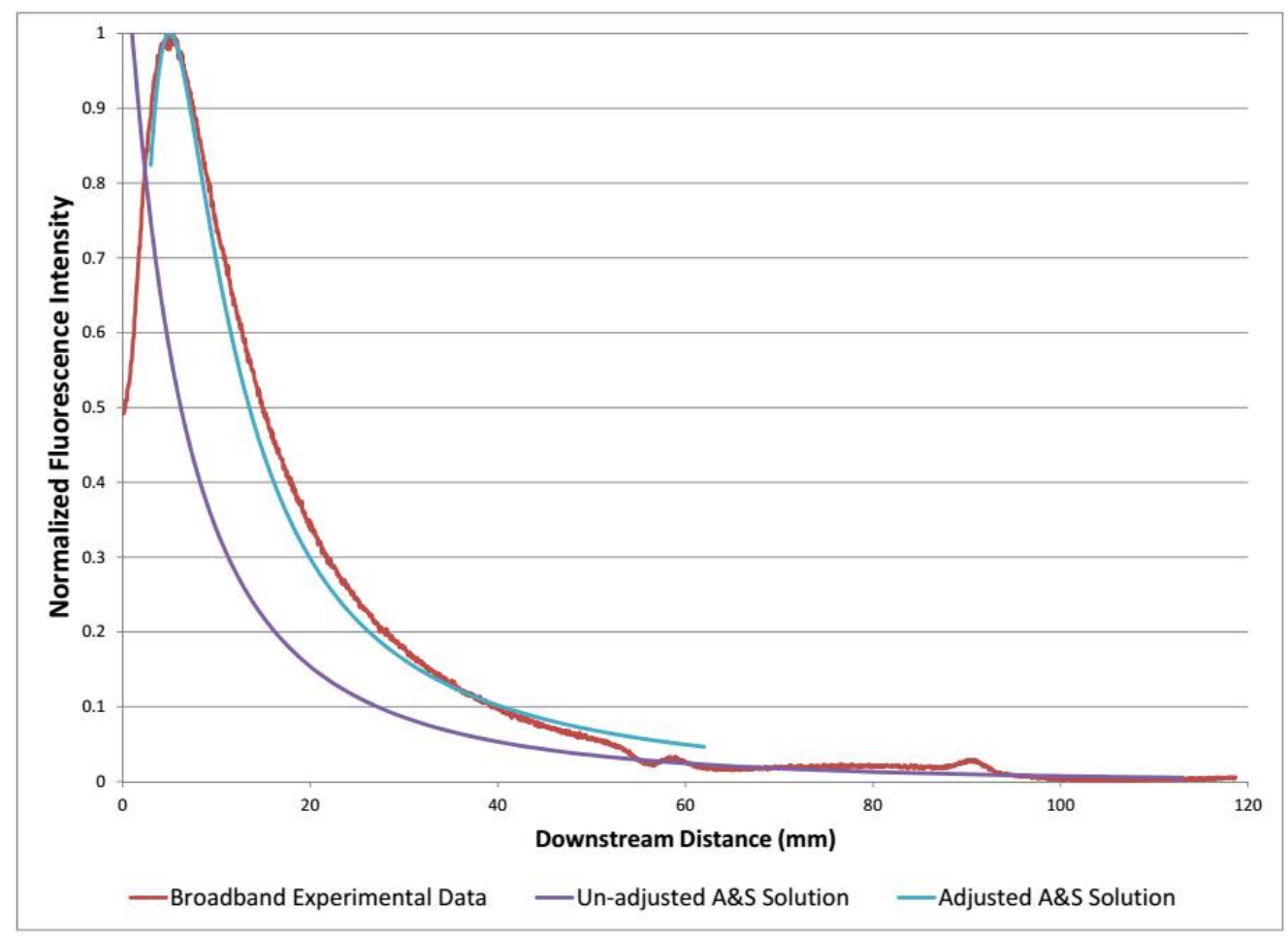

Figure 5.19: Ashkenas \& Sherman prediction vs. Centerline Fluorescence.

Contour plots from the single jet experiment at three locations within the jet core 
fluorescence were selected in order to provide specific comparison between the experimental fluorescence signal and the iodine model absorption spectrum prediction. Locations of 150,250, and 350 pixels above the top of the nozzle disk were selected for this analysis. A pixel location of 150 corresponds to $3.85 \frac{x}{D}$, pixel 250 corresponds to $6.4 \frac{x}{D}$, and pixel 350 corresponds to $8.96 \frac{x}{D}$. Figure 5.20 contains the experimental contour plots for a $P_{\text {ratio }}$ of 19550, and each pixel cross section is represented by a uniquely colored dotted line.

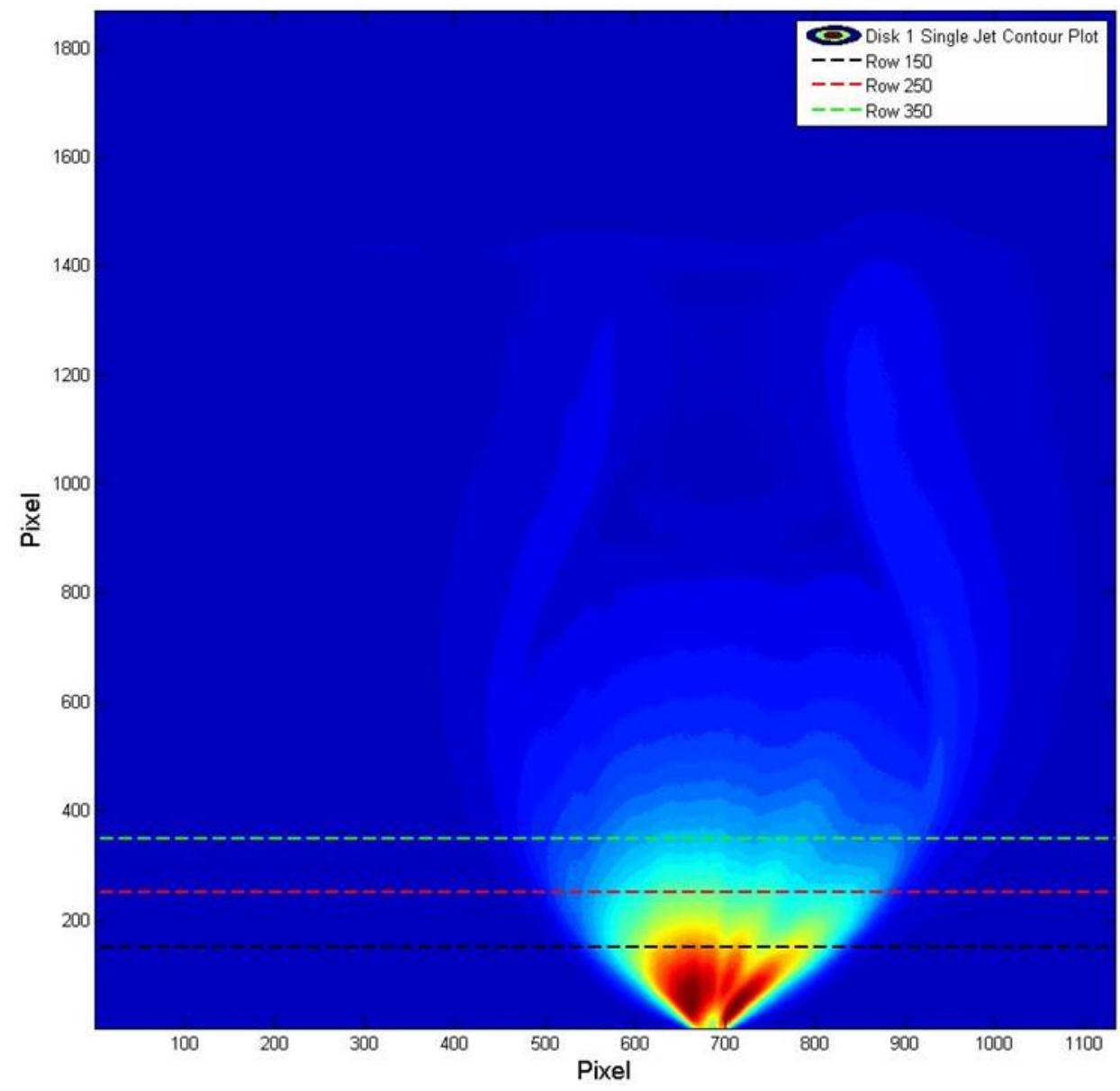

Figure 5.20: Single Jet Contour Plot.

Figure 5.21 shows the plots of fluorescence signal along each cross-section normal to the jet centerline shown in the contour image. The experimental data has had a Savitzky-Golay filter applied to smooth the noise in the line plots. The top plot corresponds to row 350 , the middle plot to row 250, and the bottom plot corresponds 
to row 150 in the fluorescence contour plot. The variation in spatial orientation of the jet core velocities relative to the laser sheet gives rise to Doppler shift in the fluorescence data, as discussed previously. This results in the resolution of the hyperfine spectra due to the spatial velocity distribution. The broadening of the spectrum is thus due to the increased angles of velocity vectors relative to the laser sheet as the flow moves downstream in the jet core.
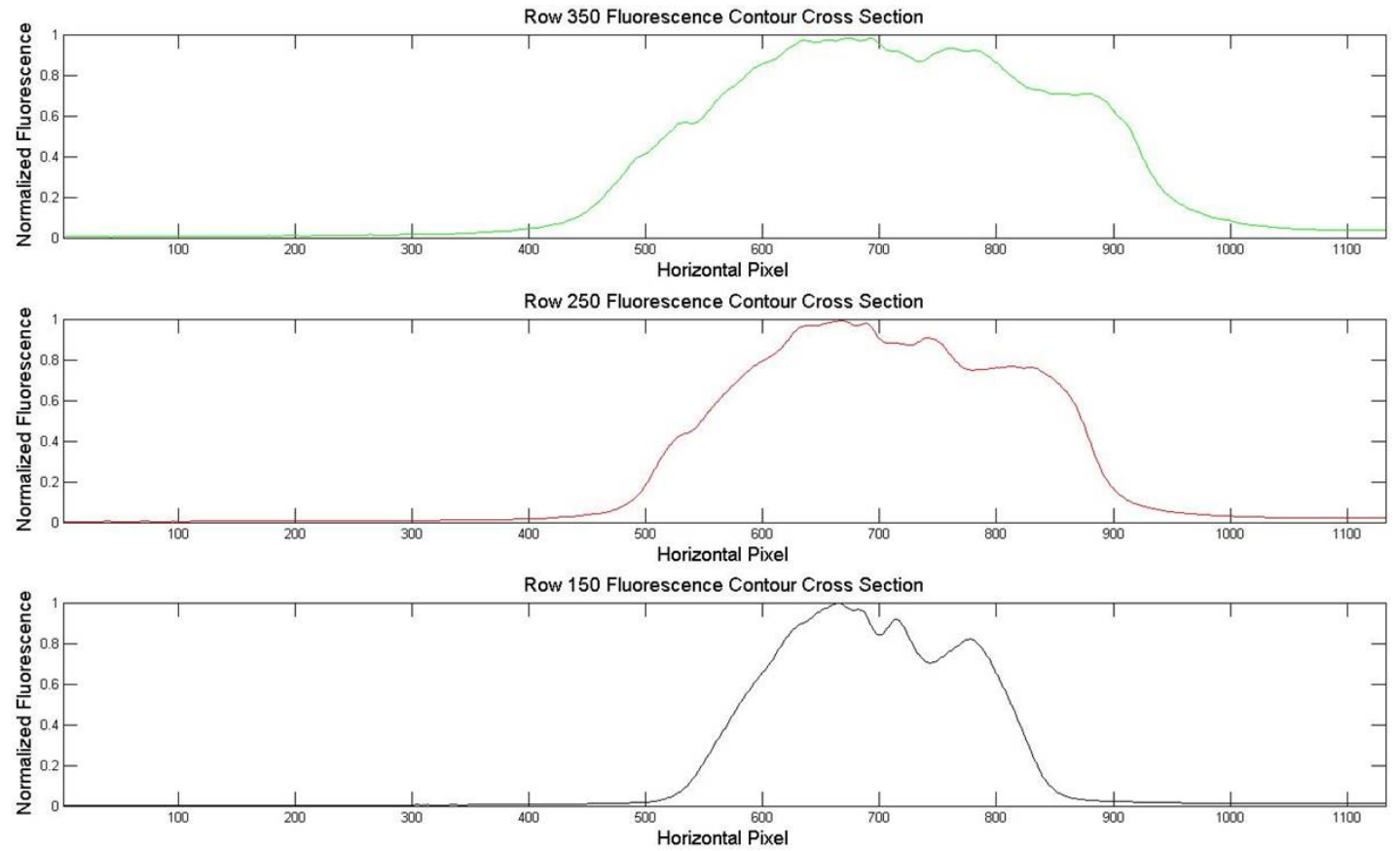

Figure 5.21: Overlay of Three Fluorescence Horizontal Cross-sections.

In Figure 5.22, a comparison of the predicted fluorescence and the experimental fluorescence at pixel $150\left(3.84 \frac{x}{D}\right)$ from Figure 5.20 is presented. Again, this comparison assumes the velocity and thermodynamic conditions normal to the centerline are the same as on the centerline, which does introduce some error in the comparison. From this image, it is clear that the predicted hyperfine spectrum correlates well with the experimental data. The location of the predicted maximum fluorescence peak corresponds directly to the location in frequency of the highest region of experimental fluorescence data. The left side peak has a minimal fluorescence signal correlation. The double peaks to the right on the other hand, correlates very well with the lo- 
cation of the fluorescence peaks in the experimental data. It is worth noting that there is some uncertainty in the location and strength of the hyperfine components because of the difficulty inherent in calculating or measuring these transitions. For reference, the hyperfine transitions used in the iodine model were shown in Figure 3.5 in Chapter 3 .

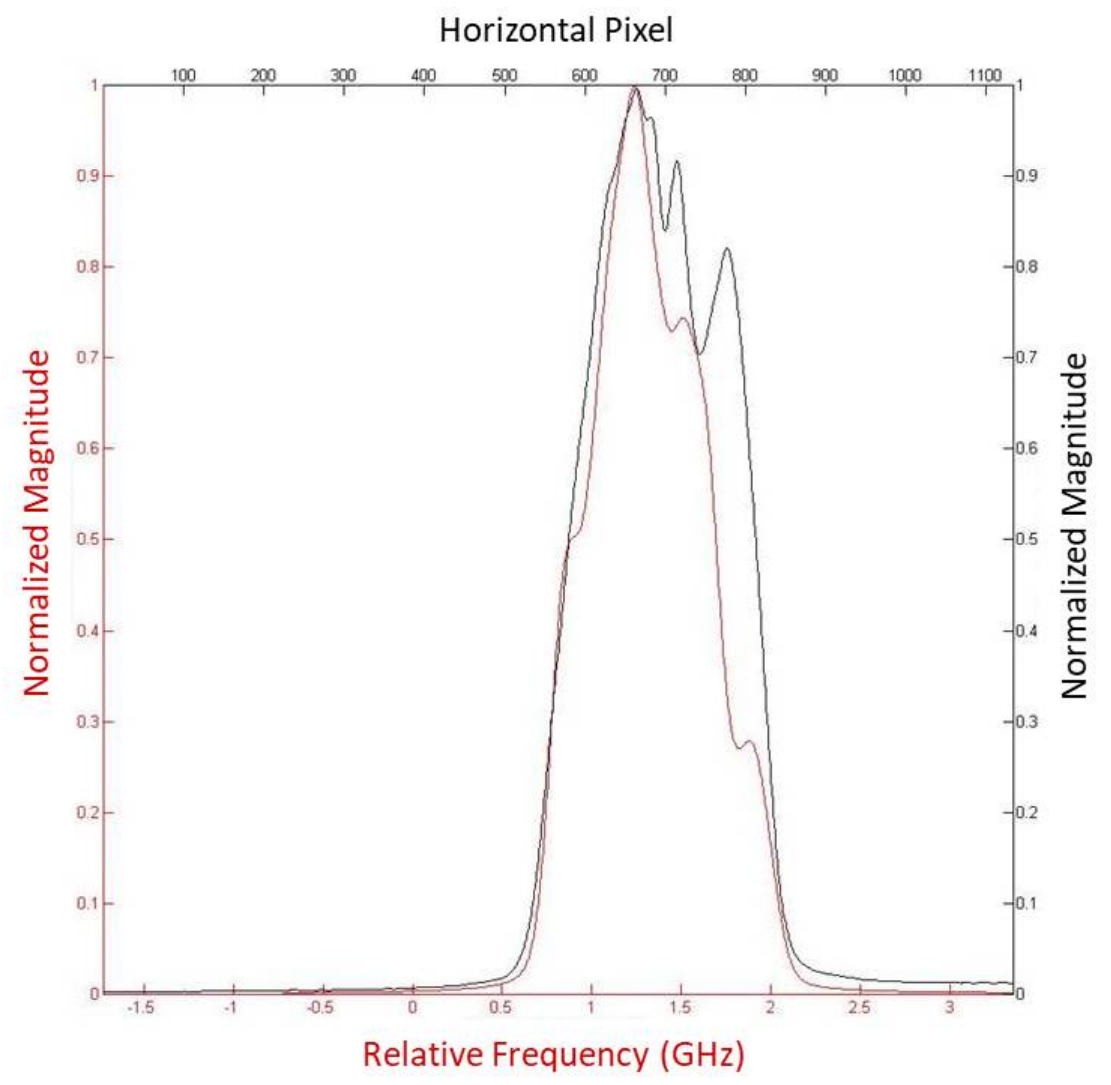

Figure 5.22: Row 150, Comparison of Predicted Spectrum and Fluorescence Signal.

Figure 5.23 shows the results for an $\frac{x}{D}$ of 6.4 . Again, the physical location of each predicted peak matches very well with the experimental data. The left side predicted peak continues to not directly correlate to an experimental fluorescence peak, but the location of the maximum and two right side peaks show very good spatial correlation. 


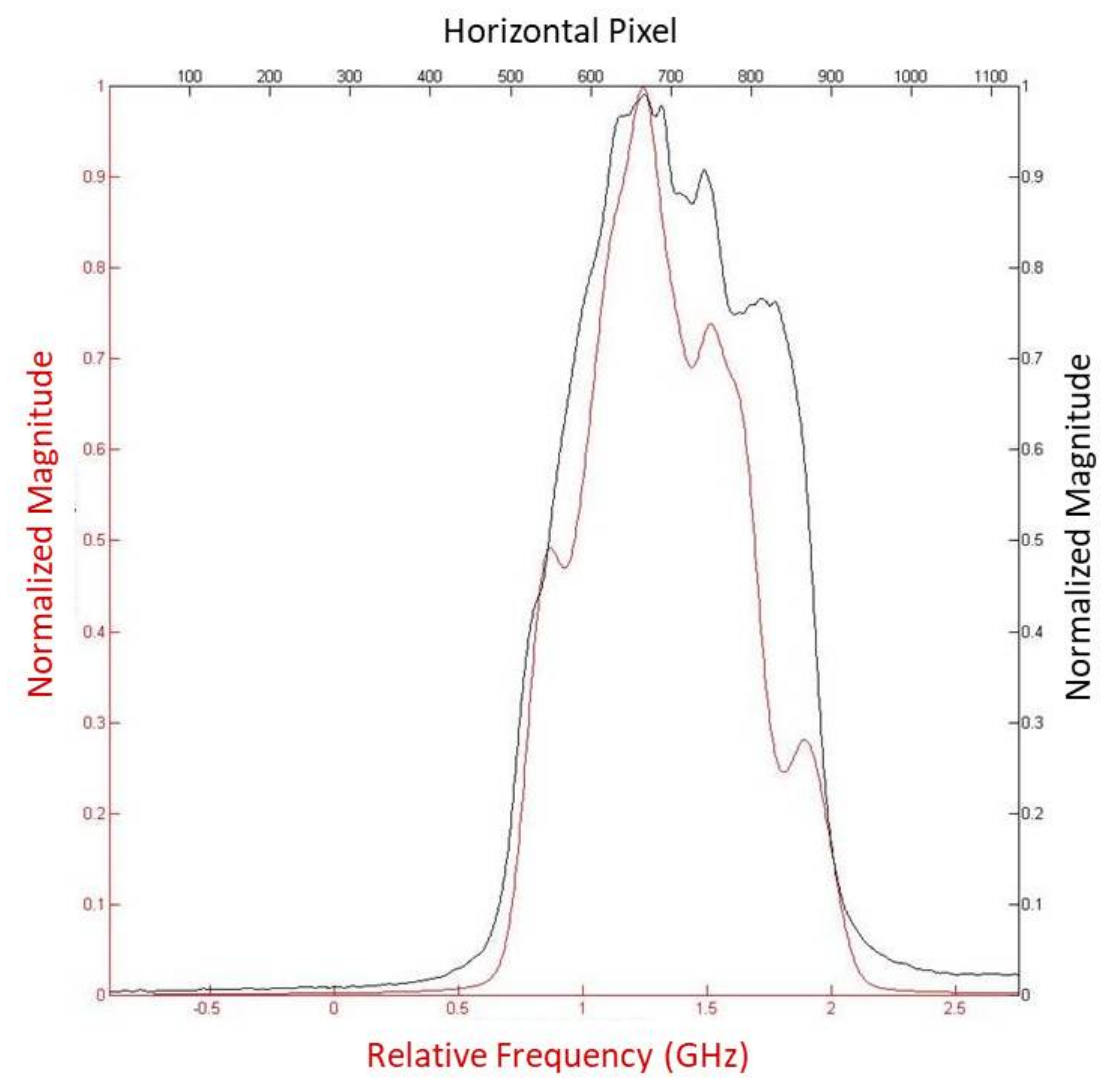

Figure 5.23: Row 250, Comparison of Predicted Spectrum and Fluorescence Signal.

Continuing downstream in the flowfield to an $\frac{x}{D}$ of 8.96 , Figure 5.24 continues to demonstrate to correlation in physical space of the hyperfine peaks. Here, the left side predicted peak begins to resolve in the fluorescence signal, while the two right side peaks begin to blend in the experimental fluorescence signal. Since this location is in a region of rarefied flow, the signal is not as finely resolved as at the upstream locations above, which can account for some of the signal blending in the experimental data. 


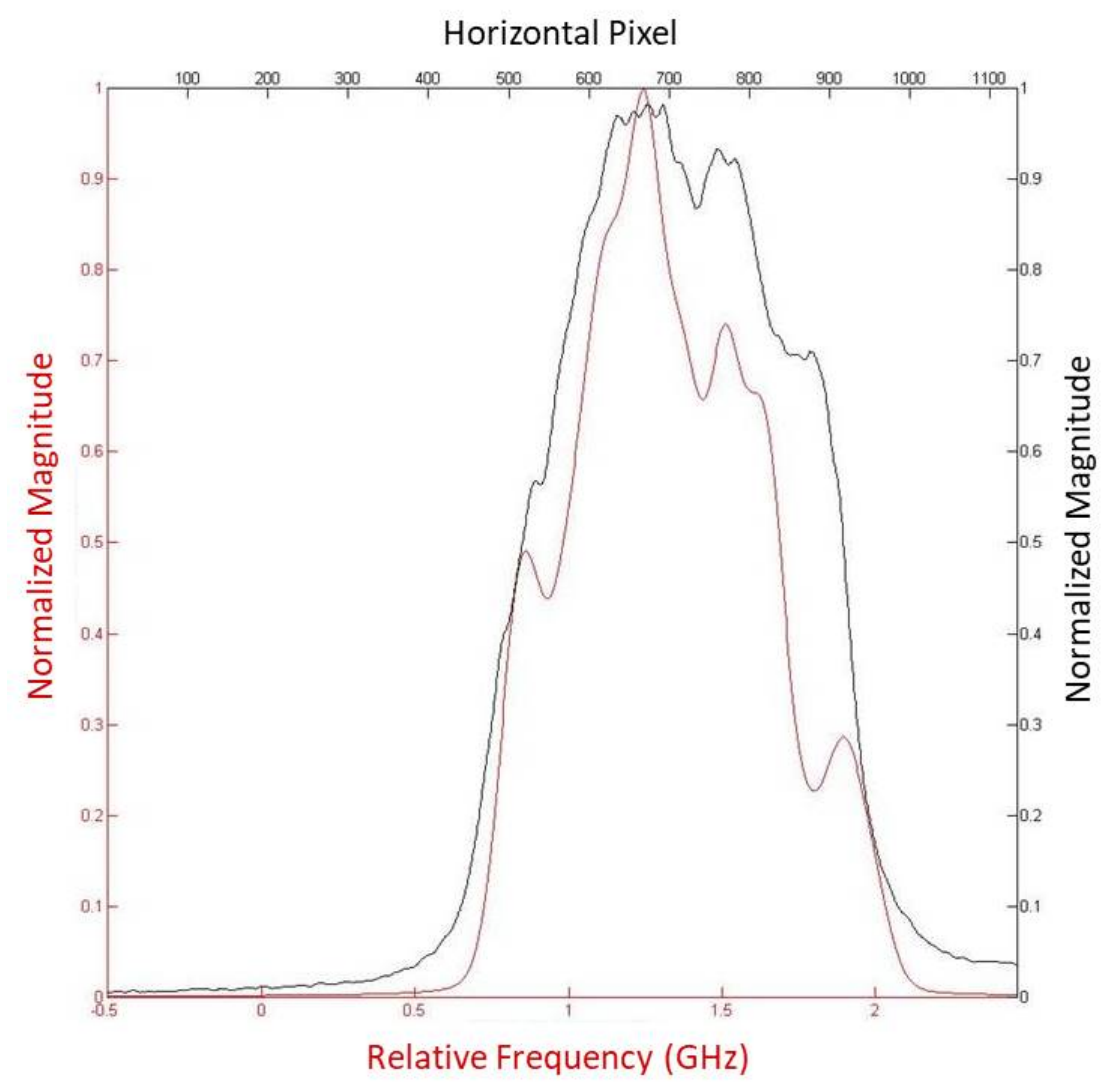

Figure 5.24: Row 350, Comparison of Predicted Spectrum and Fluorescence Signal.

While the predicted normalized magnitude does not match exactly with the experimental data, the physical location in frequency of these peaks, within the experimental core fluorescence, demonstrates that the spectroscopic phenomenon in the jet core fluorescence signal is due to the resolution of the iodine hyperfine spectrum. The iodine model script used to create the above comparisons is included in Appendix D, This script includes the relative frequency location and strength of the hyperfine components as seen in Figure 3.5 in Chapter 3 . Additional hyperfine spectrum analyses for other jet conditions are included in Appendix E.

By comparing the predicted fluorescence signal to the experimental fluorescence data for a single jet free exhaust configuration, it can clearly be seen that the variation in fluorescence intensity in the jet core can be attributed to the resolution of the 
hyperfine iodine spectrum. This spectroscopic phenomenon, along with the Doppler bias of the fluorescence signal shown at the beginning of this chapter, are two unique aspects of the PLIIF technique utilized in this research. While the hyperfine phenomenon in the jet core can be negated by adjusting the camera exposure time, the Doppler bias in the broadband fluorescence signal can only be addressed by using the single frequency technique outlined in Chapter 3. While the qualitative broadband images allow for physical geometry measurements of flow features, the broadband density ratio technique allows for a quantitative measurement of the flowfield, and will be discussed in Chapter 6 . 


\section{CHAPter 6}

\section{Results - Quantitative Density Ratio}

\subsection{Broadband Density Ratio}

The basis for the quantitative density ratio technique has been discussed previously in Chapter 3. In order to calculate the density ratio on a per-pixel basis, two broadband images are taken of the same flowfield. One image is taken with all four jets seeded with iodine. The other image is taken with a single jet seeded with iodine, and the three remaining jets seeded only with nitrogen. By taking the ratio of the single jet seeded fluorescence data by the four jet seeded fluorescence data, and scaling density ratio to make the exit density ratio one, the density ratio at each pixel in the flowfield can be calculated.

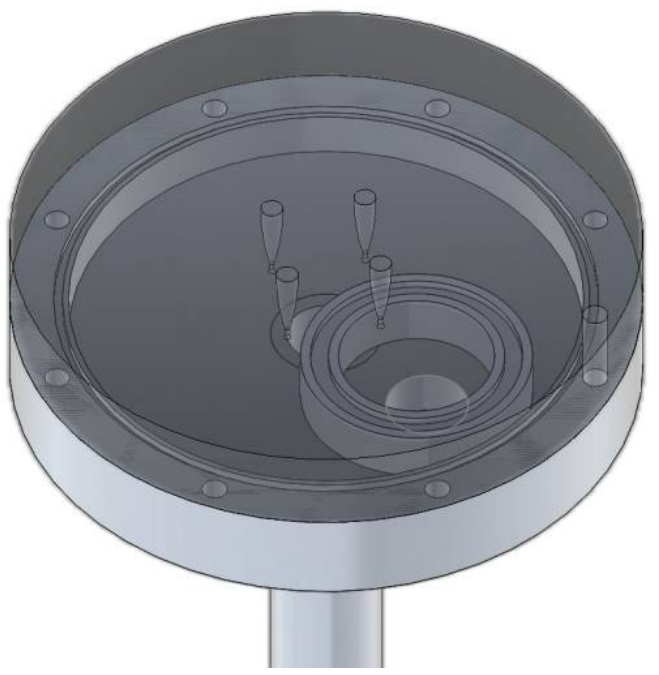

Figure 6.1: SolidWorks Model for Density Ratio Experiments. 
In order to facilitate controlling the seeding of three jets, the design of the model plenum needed to be modified. The modified SolidWorks file is shown in Figure 6.1, with the nozzle disk made transparent so the new annular plenum insert is visible. The transparent upper plate, which contains the four jet contours, also shows the alignment of the new annular plenum insert isolating a single jet nozzle contour. The original plenum design was shown in Figure 4.12. By inserting an annular secondary plenum, and including a secondary gas plumbing route, the control of iodine to three jets is made possible. The annular secondary plenum is sealed using Viton O-rings, similar to those used to seal the external diameter of the plenum. The final data collection method for density ratio experiments is outlined in Chapter 4.

\subsection{Density Ratio Contour Plots and Mixing Area}

After completing the visualization test matrix investigations, four primary test configurations were selected for density ratio investigation. Because this density ratio method measures the contribution of a single jet to the mixing and interaction with neighboring jets, Disk 1 was selected as the nozzle disk for the following experiments. The four jet, free exhaust and 3.52 inch impingement standoff were selected as the representative configurations for the four jet exhaust cases. These two cases were selected due to the similarity with the ISS flight incident that this research is based upon. The two jet cases were also investigated because this jet exhaust configuration is the current flight mode for the HTV spacecraft in the vicinity of the ISS. For the two jet investigations the free exhaust and 2.54 inch impingement standoff distance were selected. Figure 6.2 contains an example of the two component images needed to create the density ratio calculation and subsequent contour plot for a four jet free exhaust configuration. Note that some asymmetry is apparent in Figure 6.2 due to the Doppler bias. This effect is removed when the density ratio is calculated since 
both images contain the same Doppler bias.

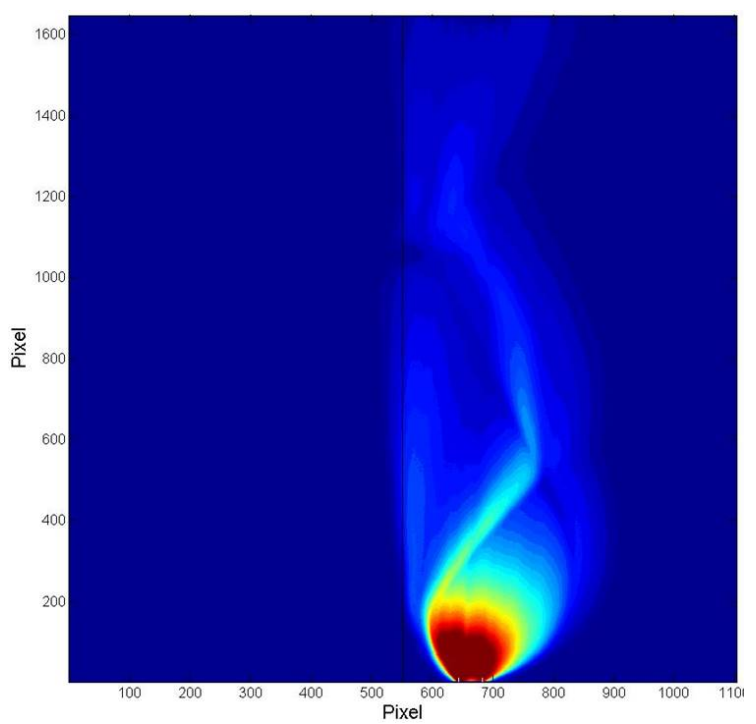

(a) Single Jet Seeded Fluorescence.

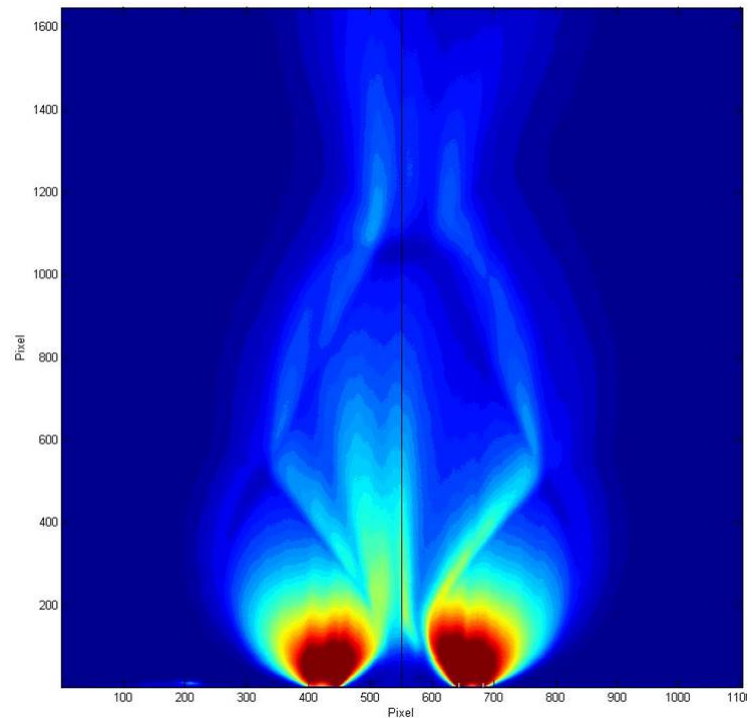

(b) Fully Seeded Fluorescence.

Figure 6.2: Component Images for Density Ratio Calculation.

From the density ratio image, the impact of neighboring jets on single jet mixing can be investigated. In order to quantify the interaction, a method for calculating the mixing area was developed. The mixing area on the density ratio image is the region where the density ratio is less than one. This represents the fact that the number density is not all from the single jet, and includes fluid from the adjacent jets. Along each horizontal pixel row an integral was calculated that represented the mixing contribution for that row. The equation used to determine the mixing integral for each row is:

$$
\text { Row Mixing Integral }=\int_{a}^{b}[1-g(x)] d x
$$

where $g(x)$ takes the form:

$$
g(x)=a_{1} e^{-\frac{\left(x-m_{1}\right)^{2}}{2 s_{1}^{2}}}+a_{2} e^{-\frac{\left(x-m_{2}\right)^{2}}{2 s_{2}^{2}}}
$$

The integral bounds $a$ and $b$ represent the beginning and ending of the mixing for the 
given row, and are shown in Figure 6.3. The start of the mixing interaction in a row is the horizontal pixel where the density ratio first exceeds 0.1 . Whereas, the end of the mixing region is the horizontal pixel where the density ratio crosses unity, i.e. a region of all single jet fluid. If the density ratio does not reach unity in a specific row, the location of the maximum density ratio is used as the end of the mixing region. For Equation 6.1, the value of one represents the upper threshold of density ratio in the mixing area, and $g(x)$ represents the density ratio data for a given horizontal row of pixels. For $g(x)$, the density ratio distribution at any given horizontal pixel row is modeled as a single or double peak Gaussian function. Within the $g(x)$ equation, $a_{1}$ and $a_{2}$ represent the density ratio value for the first and second peak, respectively. If the density ratio plot for a given pixel row only contains a single peak, the value for $a_{2}$ becomes zero. Within the exponential terms, $m_{1}$ and $m_{2}$ represent the pixel location for the first and second peak, respectively. Finally, $s_{1}$ and $s_{2}$ are the standard deviation, or the width of the peak. The equation for $g(x)$ will vary with downstream pixel, and is unique to each pixel row. 


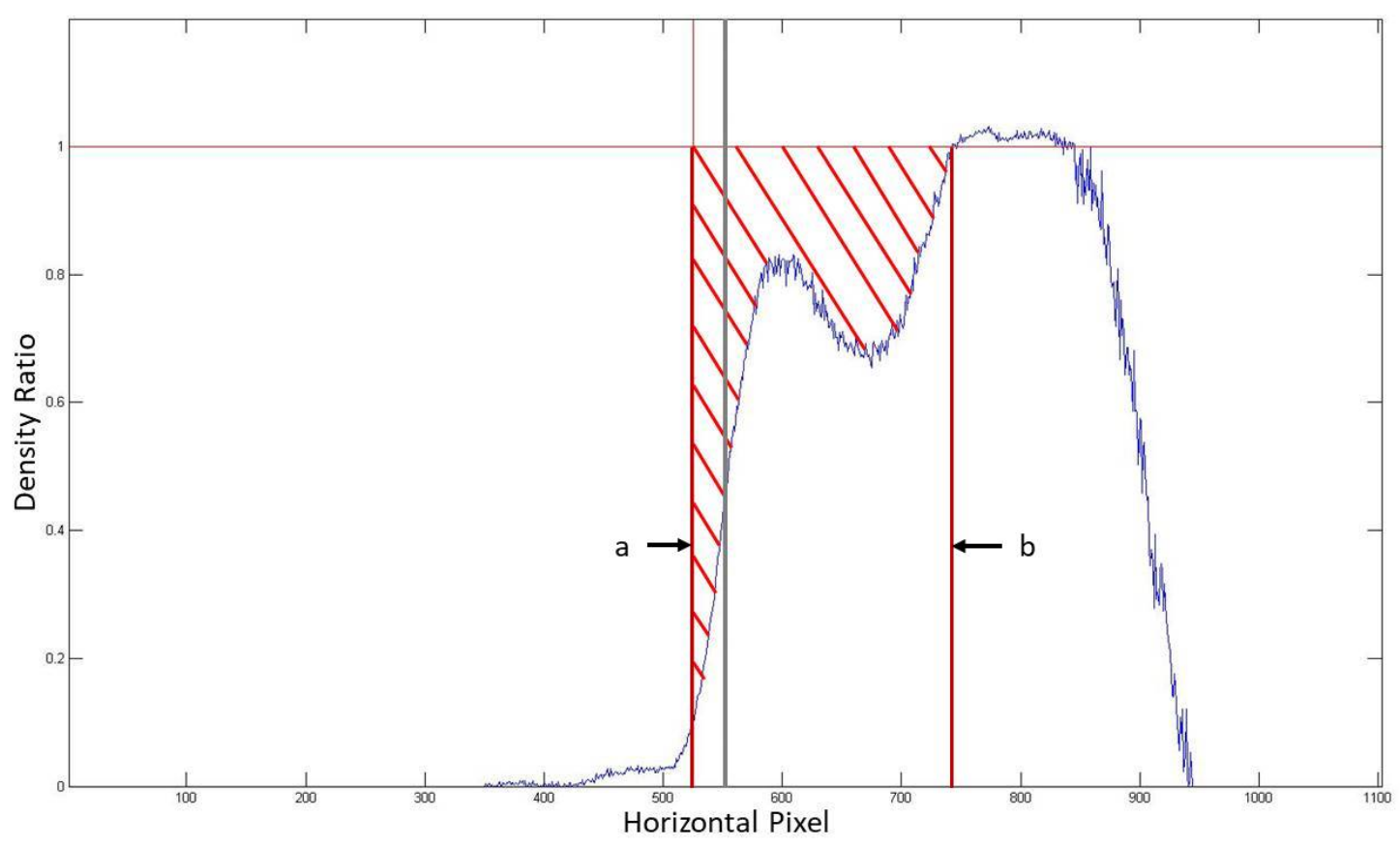

Figure 6.3: Example of Mixing Integral and Flow Integral Calculations for a Single Pixel Row.

In Figure 6.3, the centerline of the flowfield is shown at horizontal pixel 552 and is denoted by the solid black vertical line. The left vertical red line, at horizontal pixel 525, is the start of the interaction mixing region, labeled $a$. The end of the interaction mixing region is where the horizontal red line, at the density ratio of 1 , intersects with the density ratio line plot. The end of the interaction is labeled as $b$ corresponding to Equation 6.1. This intersection occurs at a horizontal pixel of 747 . The red hashed region in Figure 6.3 represents the calculation outlined in Equation 6.1 .

Next, an integral is evaluated that represents the total flow area at the given row:

$$
\text { Flow Integral }=\int_{1}^{p} g(x) d x+\int_{C L}^{b}[1-g(x)] d x
$$

In Equation 6.3, $g(x)$ is evaluated over the entire density ratio distribution, where $p$ is the final horizontal pixel in the vertical pixel row. This region is shown in Figure 6.3 by the blue line plot. The equation $g(x)$ is defined in Equation 6.2. The 
second integral is evaluated from the flow centerline, $C L$, to point $b$ and represents the additional possible flow area contribution by a single jet if there is no mixing, or neighboring jet interaction, present. By evaluating the ratio of the Flow Integral to the Row Mixing Integral at each row, a measure of the mixing percentage at each downstream pixel row can be calculated. This can be written as:

$$
\text { Mixing Percentage }=\frac{\text { Row Mixing Integral }}{\text { Flow Integral }}
$$

Once these calculations have been completed for each downstream pixel, the total mixing percentage can be calculated as a ratio of the total Row Mixing Integral values divided by the total Flow Integral values for the entire flowfield. This ratio is written as:

$$
\text { Mixing Percentage }_{\text {Total }}=\frac{\sum_{1}^{n} \text { Row Mixing Integral }}{\sum_{1}^{n} \text { Flow Integral }}
$$

Equation 6.5 contains the ratio of the sums of the Row Mixing Integral and the Flow Integral, evaluated from pixel 1 to $n$, where $n$ is the final pixel in the CCD camera field-of-view. Equation 6.5 represents a measure of the amount of mixing from unseeded jets versus the contribution to the flowfield from the single seeded jet. This can be thought of as a measure of the amount of mixing in a given flowfield configuration.

The first density ratio experiment consists of testing Disk 1 in the four jet, free exhaust configuration. The plenum pressure was established at 25 psia, with a back pressure of 131.6 millitorr. This resulted in a $P_{\text {ratio }}$ of 9824.

A side-by-side comparison of the single jet fluorescence contour and the density ratio contour is presented in Figure 6.4. The density ratio calculation was performed by dividing the single jet fluorescence data by the fully seeded flow fluorescence data, 
an example of which is shown in Figure 6.2, an normalized by the value of the density ratio at the single jet exit. This normalization is performed based on the fact that the density ratio at the jet exit is known to be solely from the fluid exiting that jet, which means the density ratio for this part of the flow is unity. It is also interesting to note that the hyperfine splitting of the iodine fluorescence in the jet core, as explained in Chapter 5, does not appear in the density ratio image since the density at those locations are all from the single jet, which means the density ratio is equal to unity everywhere in the jet core.

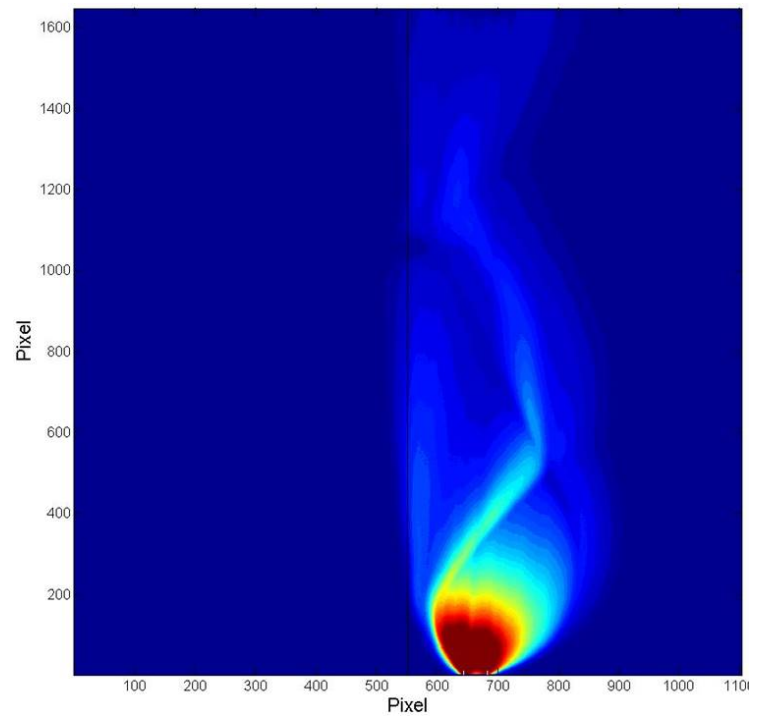

(a) Single Jet Seeded Fluorescence.

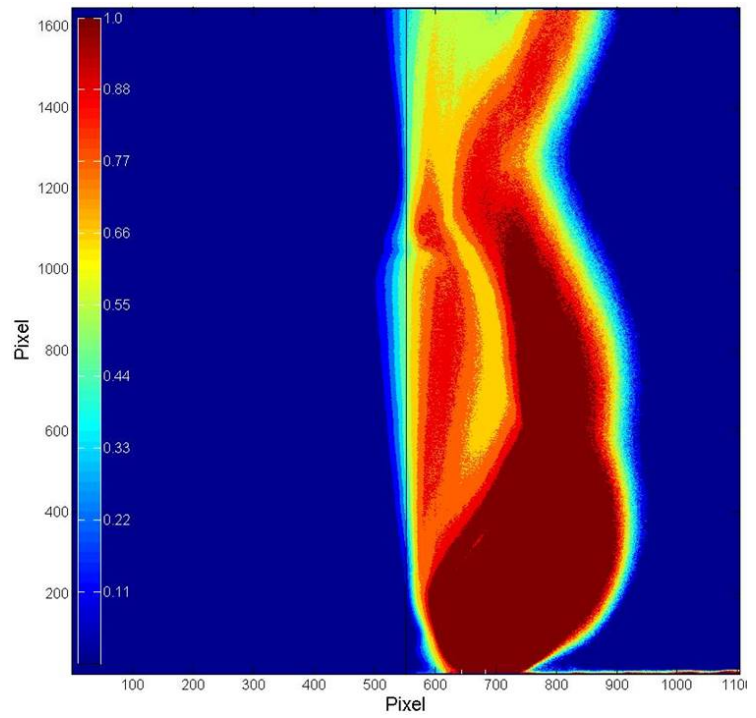

(b) Density Ratio.

Figure 6.4: Single Jet Seeded versus Density Ratio Contour Plots, for 4 jet, free exhaust.

In Figure 6.5, the density ratio image, with mixing boundaries, can be seen. Within this image, the line representing the integral $a$ boundary in Equation 6.1 is shown in red, and the $b$ values from the same integral are shown in black. It can be seen that the jet core has a density ratio of unity, which indicates the boundaries of the purely jet core flow. 


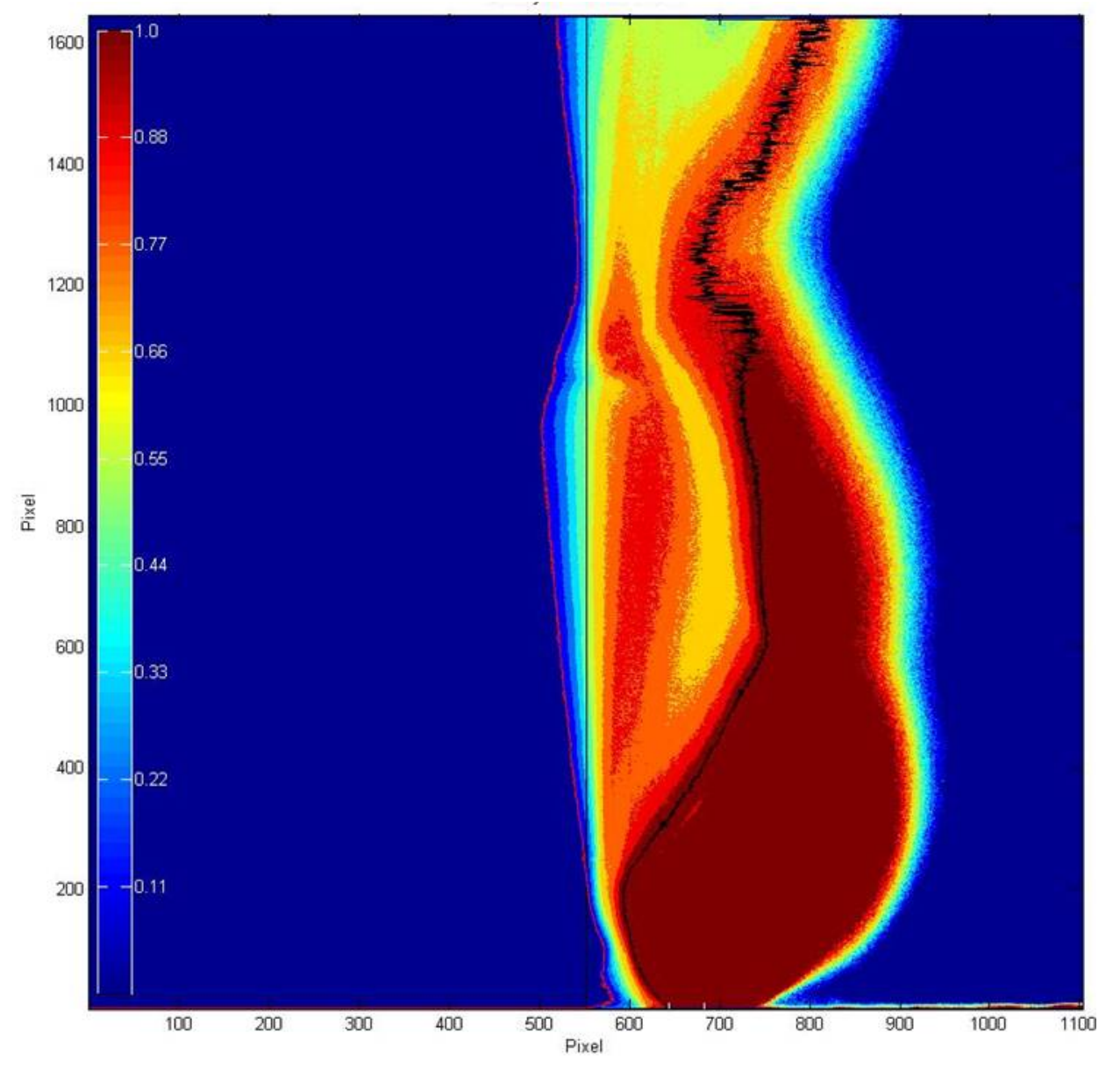

Figure 6.5: Density Ratio image - 4 Jet Free Exhaust.

The jet core flow persists until close to the flow feature (indent) in the center of the mixing at around vertical pixel $1100\left(28.1 \frac{x}{D}\right)$. In the discussion of density ratio images, the nomenclature 'flow feature' is used since it is not clear in the density ratio images what this represents. A 'flow feature' does not indicate the presence of a shock since the density ratio does not change across a shock. It is also worth noting that the flow features for all cases presented occur near the flowfield centerline. Proceeding downstream from this flow feature, the density ratio drops below unity, indicating that mixing is occurring farther into the right portion of the core flow. Between vertical pixel 200 and 210, 5.1 and $5.4 \frac{x}{D}$ respectively, the mixing area starts, and begins to rapidly widen. Between vertical pixel 210 and 1100, the mixing interaction is evident, both from density ratios lower than unity and the deflection of the core 
fluid. Downstream from the flow feature along the centerline, starting at vertical pixel 1100, the mixing interaction occurs across the majority of the flowfield and no solely jet core fluid flow is present.

Solving Equations 6.1 and 6.5. Figure 6.6 can be plotted. Figure 6.6 represents the mixing percentage at each downstream (vertical) pixel. It should be noted that all mixing percentage plots are presented using a Savitzky-Golay moving average filter in order to make the plots more readable (all calculations used un-filtered experimental results). The increase in mixing percentage from pixel 200 through pixel 1000, $5.1 \frac{x}{D}$ to $25.6 \frac{x}{D}$ respectively, corresponds to the deflection of the core flow and increase in mixing area seen in the density ratio image. Above pixel 1000, a drop in percentage followed by a sharp increase corresponds with the marked change in mixing following the flow feature. Downstream from the flow feature the entire flowfield demonstrates mixing, as is evidence by the density ratio in the flow all being below unity. The total mixing percentage, according to Equation 6.5, for the flowfield is approximately $19.7 \%$ at the most downstream pixel location. 


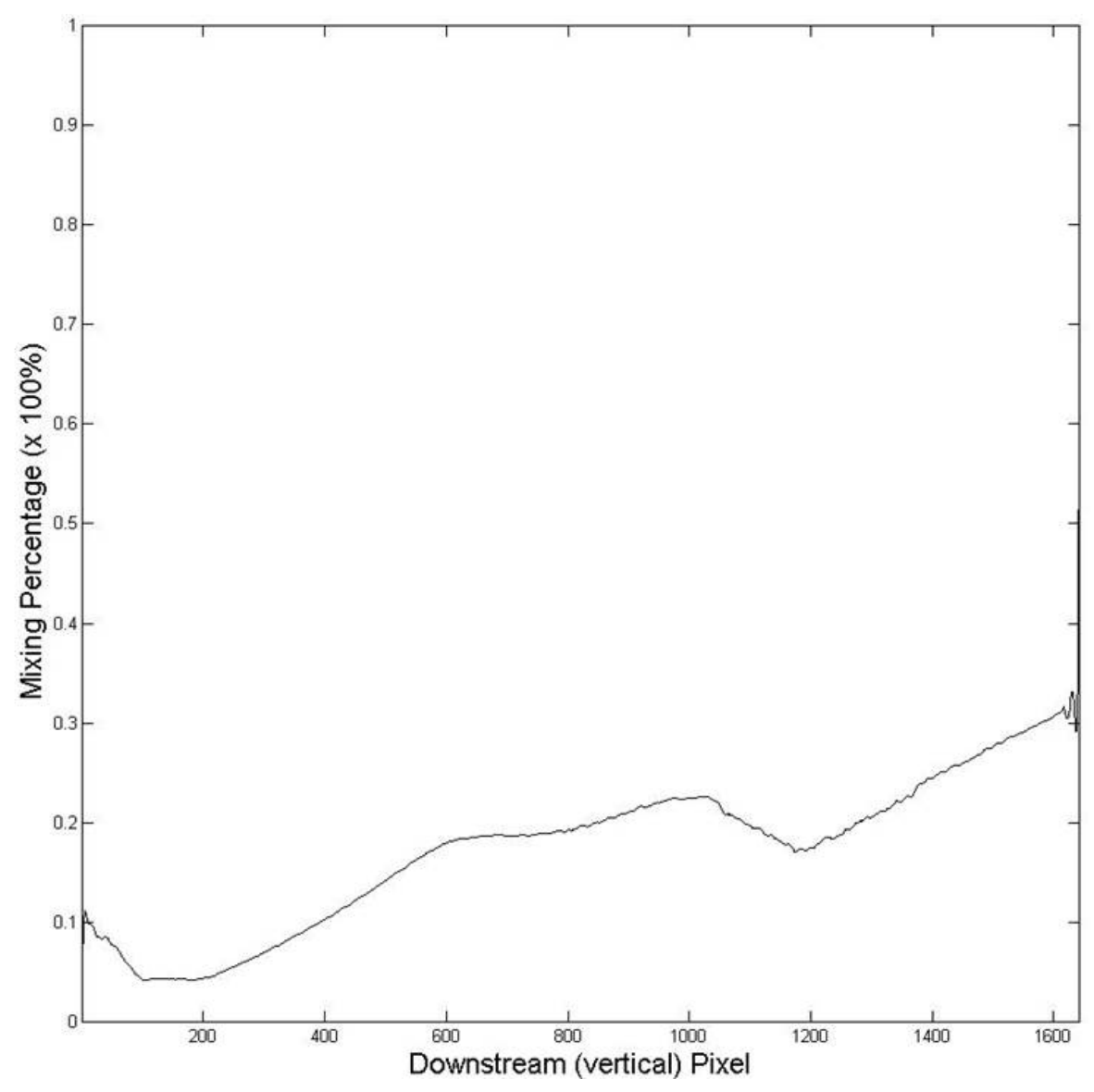

Figure 6.6: Mixing Percentage at each downstream pixel.

In order to quantify the effect on the mixing area imposed by an impingement surface, an experiment with the impingement surface standoff of 3.52 inches, or $36 \frac{x}{D}$, was conducted. The plenum pressure was set to 42 psia and the back pressure was 208.1 millitorr. The $P_{\text {ratio }}$ is 10437 , which is not only comparable to the free exhaust experiment, but also to the 3.62" visualization experiment in Figure 5.3 .

Figure 6.7 shows the fluorescence and the density ratio contour images for the four jet, 3.52 inch impingement standoff experiment. The side-by-side comparison helps clarify certain features seen in each image, allowing for a side-by-side comparison of flow features and density ratio. By comparing the fluorescence image to the density ratio image, the mixing region and impingement surface interaction becomes much clearer. The density ratio image in Figure 6.7b shows an interesting mixing feature 
between the flow centerline and the unity jet core fluid flow, starting around pixel 1100 and proceeding to the impingement surface at vertical pixel 1405.

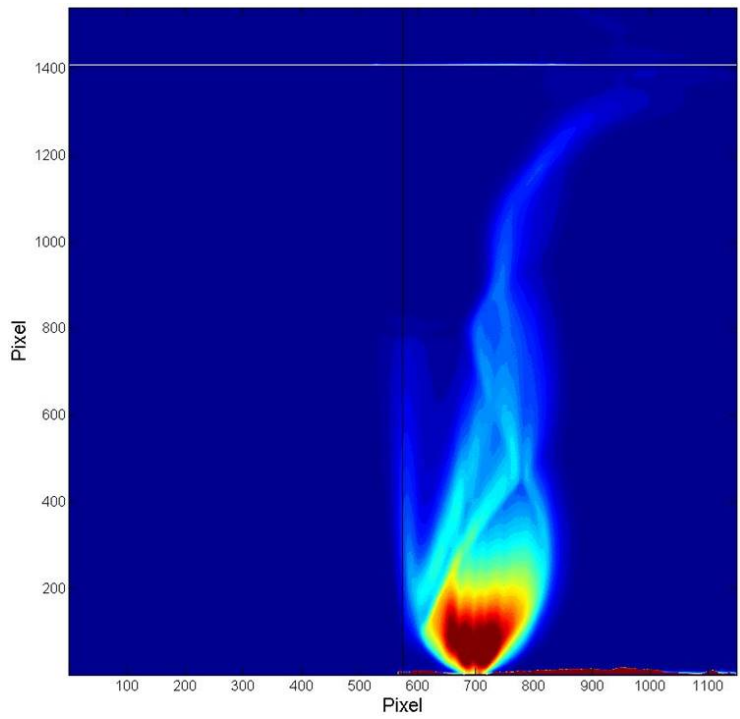

(a) Single Jet Seeded Fluorescence.

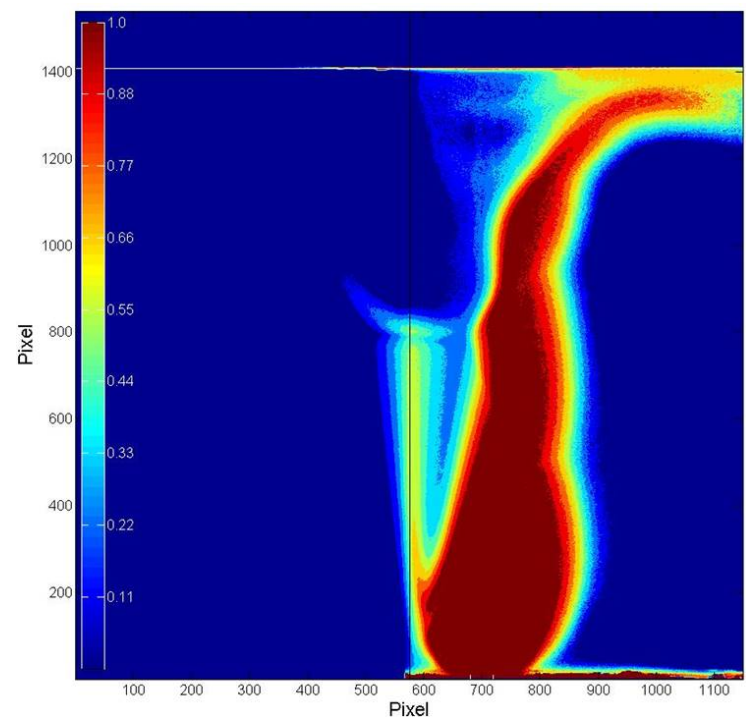

(b) Density Ratio.

Figure 6.7: Single Jet Seeded versus Density Ratio Contour Plots for 4 jet, 3.52" Standoff Distance.

In Figure 6.8, the line representing the integral $a$ boundary in Equation 6.1 is shown in red, and the $b$ values from the same integral are shown in black. The image follows a similar flow structure to the four jet free exhaust experiment, though the mixing area and presence of a new flow feature near pixel 800, as seen in the fluorescence image in Figure 6.7a, are significant differences. Note that any wave structure in the jet core is not evident in the density ratio images because the density ratio does not change across a shock or expansion wave. 


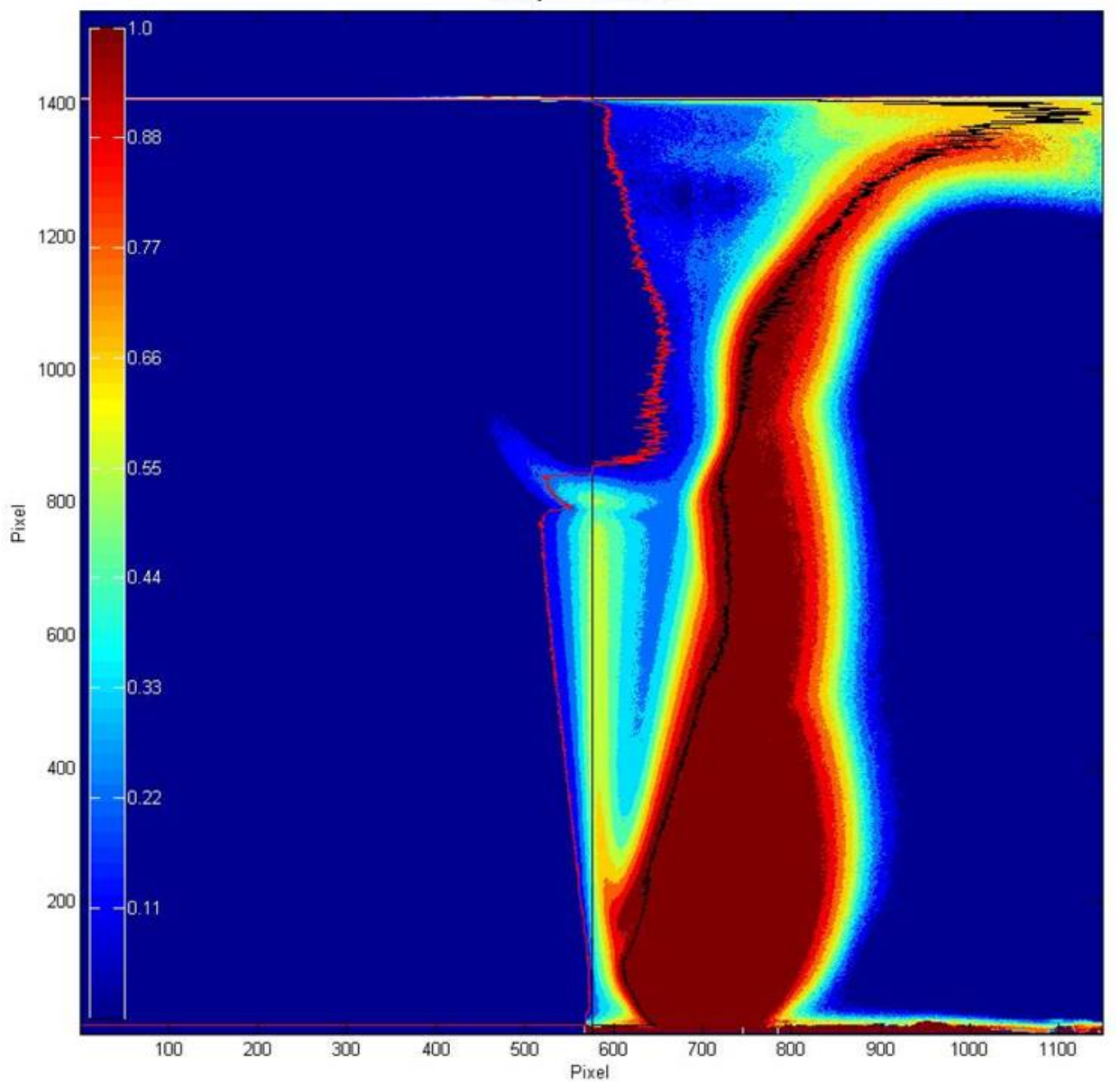

Figure 6.8: Density Ratio Image - 4 Jet, 3.52" Standoff.

The unity values for the jet core fluid also progress much further downstream than in the free exhaust experiment. The effect of the new flow feature, as seen in the linear fluorescence image in Figure 6.7a at around pixel $800\left(20.5 \frac{x}{D}\right)$, marks a sudden change in the mixing area downstream. Also, as seen in the free exhaust experiment, the density ratios in the far downstream regions do not reach unity, which indicates contributions from the other jets and mixing of the fluid flow. In the mixing region upstream of the new flow feature, seen in Figure 6.7a between pixel 200 and 800, the density ratios are not as high as the free exhaust experiment, 0.55 for this experiment and 0.7 for the free exhaust. Though the density ratios do vary between the two experiments, the structure of the mixing is very similar. Following the flow feature, as seen in Figure 6.7a, the width of the mixing region increases significantly, which 
demonstrates a large downstream contribution to mixing induced by the interaction with the impingement surface. Turning of the flow can be seen starting at the flow feature and continuing until the flow becomes nearly horizontal at the impingement surface located at pixel 1405, or $36 \frac{x}{D}$.

Following the same calculation method as the free exhaust experiment, the mixing percentage for the impingement experiment is calculated. Figure 6.9 represents the mixing percentage at each downstream (vertical) pixel. Similar to the four jet free exhaust experiment, from pixel 200 to 800 (5.1 and $20.5 \frac{x}{D}$, respectively) the mixing percentage sharply increases. This corresponds to the deflection of the core flow as soon in Figure 6.7a. At around pixel 800, $20.5 \frac{x}{D}$, there are sharp changes in the mixing percentage, which can be seen to correspond to the location of the flow feature in Figure 6.7a. Following the flow feature, at around pixel 1050 (26.9 $\left.\frac{x}{D}\right)$, the mixing percentage is seen to rise sharply. This corresponds to the large mixing area increase and the core flow size decreases post-flow feature. This points to significant mixing contributions from the unseeded jets, resulting in a highly mixed flow as the fluid approaches the impingement surface. The total mixing percentage at the most downstream pixel, which corresponds to the impingement surface at pixel 1405, for this flow is $29.7 \%$, as calculated by Equation 6.5 . 


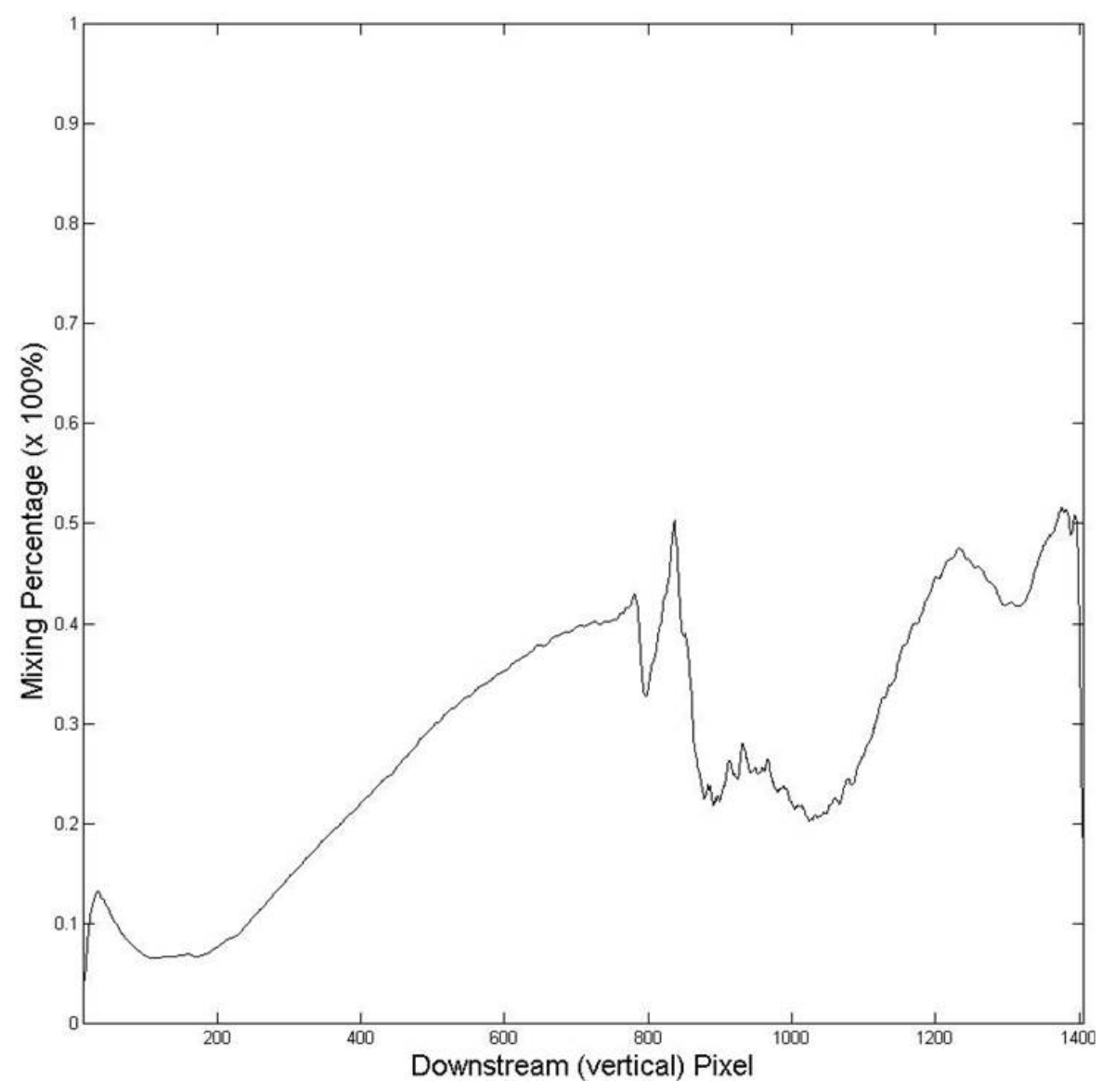

Figure 6.9: Mixing Percentage at each downstream pixel.

When the total mixing percentage at the impingement surface is compared between the free exhaust and impingement configuration, a direct quantification of the contribution of the impingement surface to mixing can be made. The total mixing percentage in the free exhaust case, at the impingement surface and calculated by Equation 6.5, is 17\%. Comparing that to the total mixing percentage of the impingement case, $29.7 \%$, an approximately $70 \%$ increase in mixing percentage can be attributed to the introduction of the impingement surface.

Following the results of the investigation into the effect of the impingement surface on the mixing area of the four jet flowfield, experiments were conducted to quantify the effect on the two jet flowfield. The two jet, free exhaust experiment was conducted with a plenum pressure of 48 psia, and a back pressure of 163.1 millitorr. This results 
in a $P_{\text {ratio }}$ of 15220 . When compared with the visualization experiment $P_{\text {ratio }}$ of 16310 , the close agreement of the pressure ratio in the two jet free exhaust experiment is indicated.

Figure 6.10 contains the fluorescence and the density ratio images for the two jet free exhaust experiment. The fluorescence data side-by-side with the density ratio, allows for comparison between flow features evident in the fluorescence image, and corresponding density ratio quantitative data in the density ratio image.

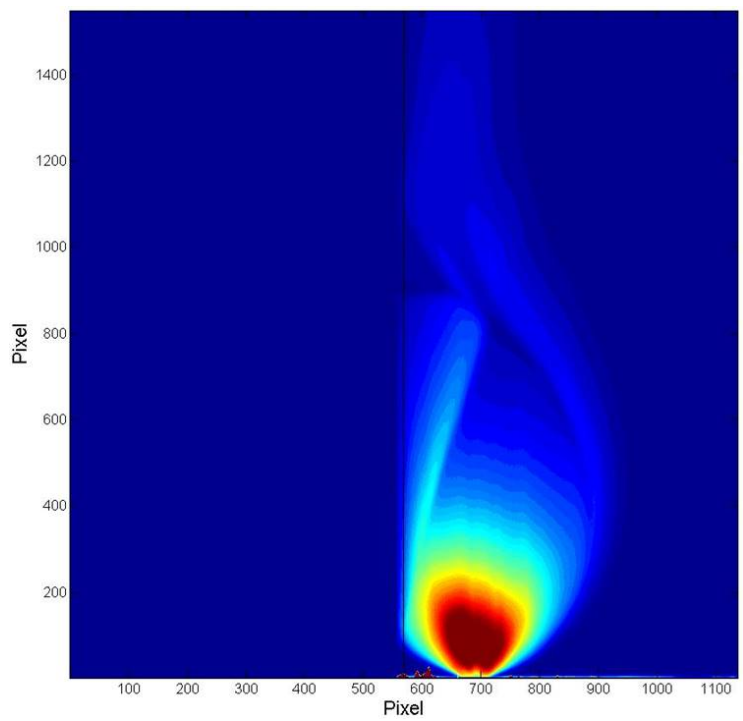

(a) Single Jet Seeded Fluorescence.

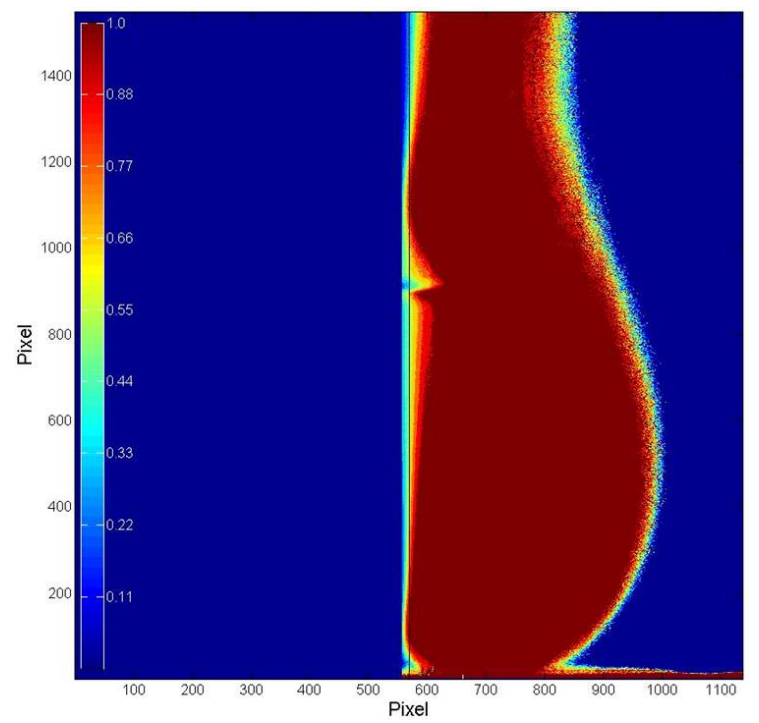

(b) Density Ratio.

Figure 6.10: Single Jet Seeded Fluorescence versus Density Ratio Images for 2 jet free exhaust.

The presence of a flow feature along the centerline, at approximately 900 pixels (23 $\frac{x}{D}$ ), is evident in Figure 6.10a. It is also interesting to note in this comparison that the fluorescence data shows a drop in fluorescence in the jet core flow, while the density ratio, correctly, shows a value of unity for the entire region of jet core flow. This indicates that the majority of the flowfield in the two jet free exhaust case is jet core fluid, and demonstrates very little mixing. 


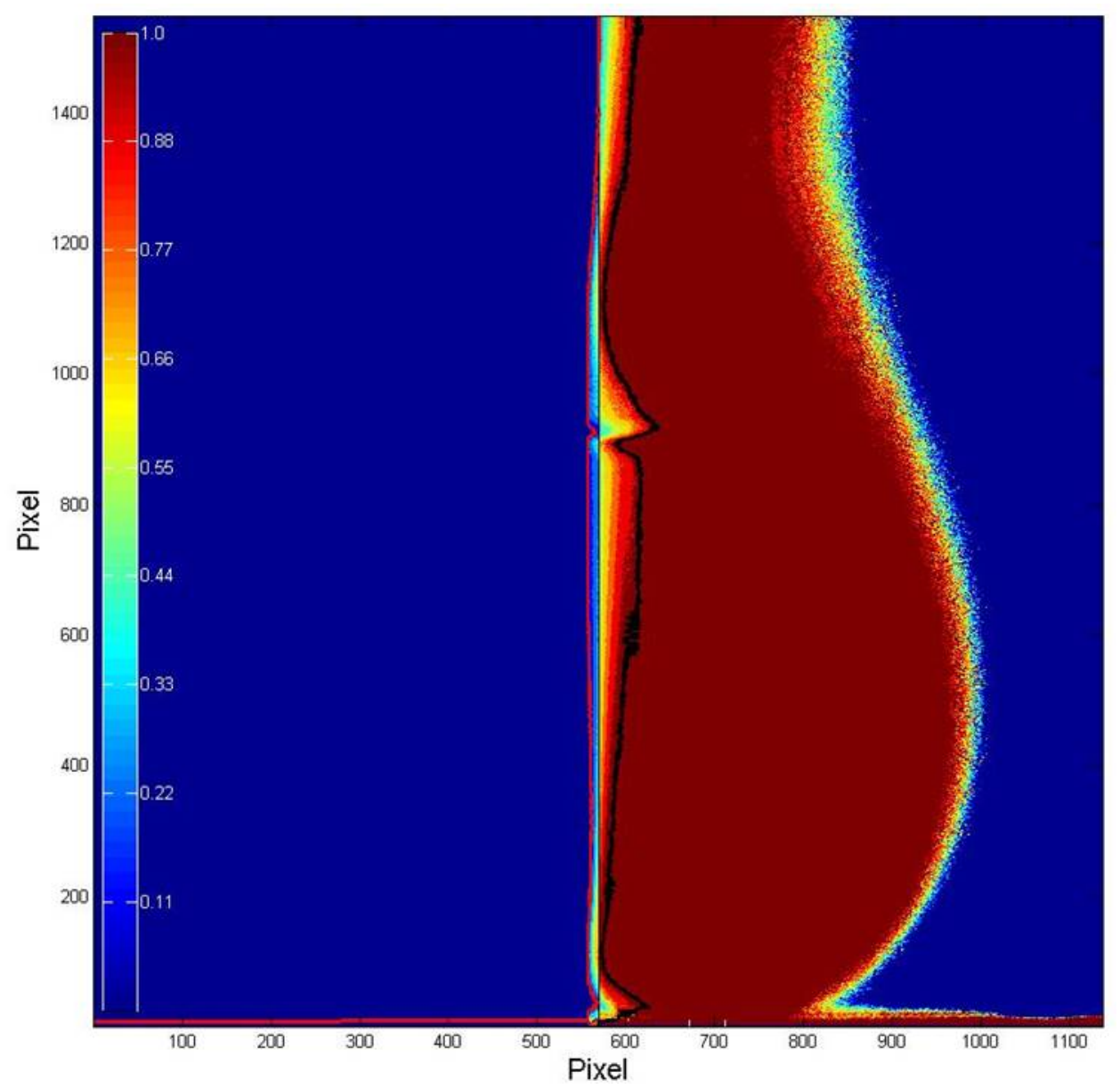

Figure 6.11: Density Ratio Image - 2 Jet, Free Exhaust.

Within Figure 6.11, as with the other density ratio images, the line representing the integral $a$ boundary in Equation 6.1 is shown in red, and the $b$ values from the same integral are shown in black. While the start of the mixing area demonstrates little deflection of the jet core fluid, there are in fact small variations to the contour. The unity density ratio region, indicating jet core fluid flow, is much larger than that of the four jet free exhaust core fluid region. According to the density ratio image, this jet core fluid flow progression downstream appears to be due to the decreased size of the neighboring jet interaction region. The initial neighboring jet interaction occurs between vertical pixels 75 and $100,1.9$ and $2.6 \frac{x}{D}$ respectively, and leads to the increase in mixing area from vertical pixel 100 to the location of the flow feature along the centerline between vertical pixel 900 and 950, 23 and $24.3 \frac{x}{D}$ respectively. 


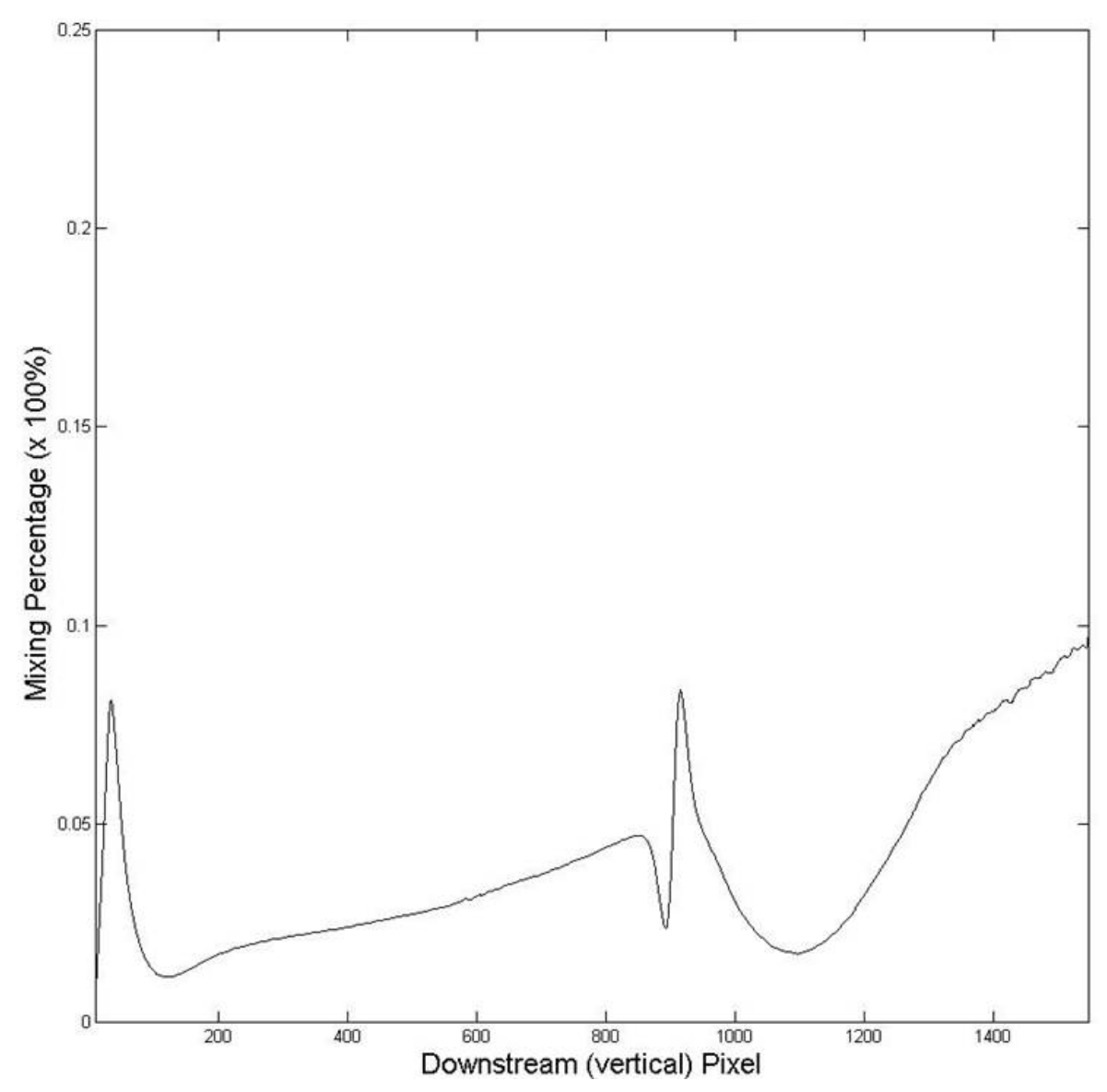

Figure 6.12: Mixing Percentage at each downstream pixel.

Figure 6.12 represents the mixing percentage at each downstream (vertical) pixel. The shape of the mixing percentage plot is significantly different than either of the four jet experiments. After the initial interaction at downstream (vertical) pixel 100 $\left(2.6 \frac{x}{D}\right)$, the mixing percentage gradually increases until the abrupt change in mixing percentage around the location of the flow feature. Between downstream pixel 900 and 950 (23 and $24.3 \frac{x}{D}$ ), the mixing percentage changes abruptly before recovering and continuing to increase as the flow progresses downstream. It should be noted that the mixing percentage for the two jet free exhaust case is approximately six times smaller than the four jet free exhaust mixing percentage. The total mixing percentage for the four jet free exhaust is $19.7 \%$, whereas the total mixing percentage for the two jet free exhaust case is $3.7 \%$, as computed using Equation 6.5. 
In order to quantify the effect on the mixing area in the two jet case caused by interactions with an impingement surface, an experiment with two jets impinging on a flat surface was conducted. For this experiment, the impingement surface was placed at 2.54 inches, or $26 \frac{x}{D}$, downstream of the model surface. The plenum pressure was set to 45 psia, resulting in a back pressure of 158.7 millitorr during the experiment. These operating conditions yielded a $P_{\text {ratio }}$ of 14664 . The two jet, 2.875 inch standoff visualization experiment had an experimental back pressure of 15422 , which verifies the repeatability of the pressure ratio of the two jet impingement experiments conducted in this research.

Figure 6.13 contains the side-by-side comparison of the single jet fluorescence density image and the density ratio image for two jets and an impingement surface with a standoff of 2.54 inches. This figure allows for comparison between flow features evident in the fluorescence image, and corresponding density ratio quantitative data in the density ratio image.

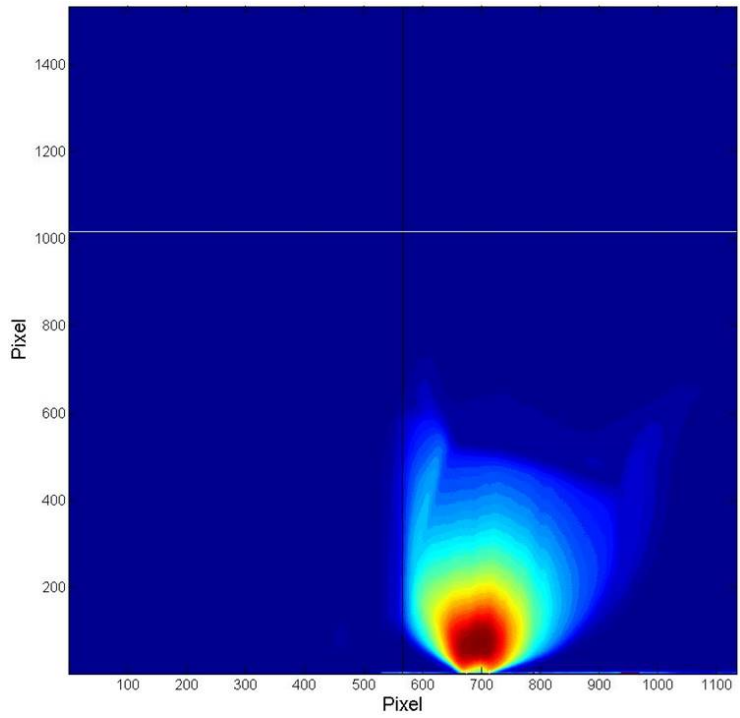

(a) Single Jet Seeded Fluorescence.

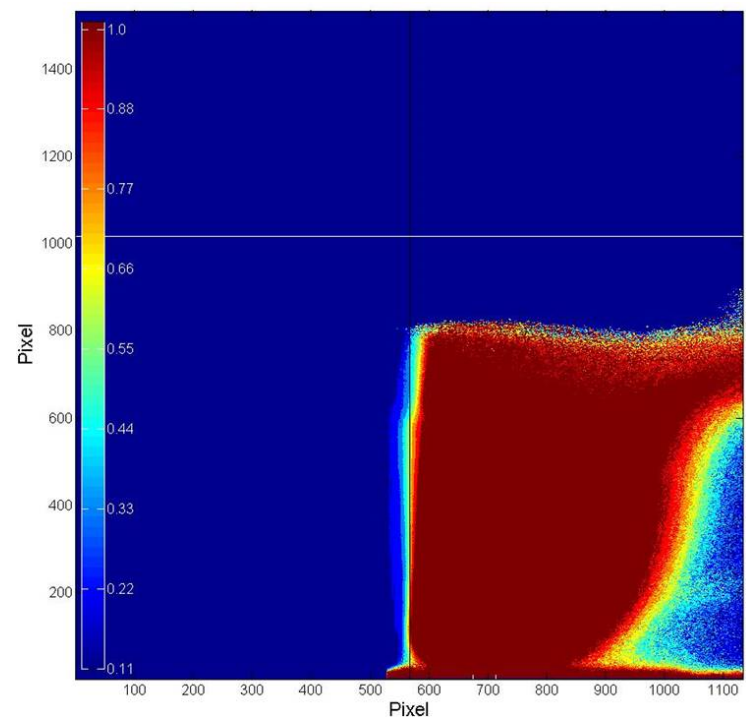

(b) Density Ratio.

Figure 6.13: Single Jet Seeded Fluorescence versus Density Ratio Images for 2 Jets Impinging on a Surface at 2.54".

The presence of a shock wave at approximately pixel $550\left(14.1 \frac{x}{D}\right)$, is evident in 
Figure 6.13a. The fluorescence data shows a sharp drop in fluorescence in the jet core flow across the normal shock due to pressure rise and increased collisional quenching, while the density ratio shows a value of unity for the entire region of jet core since the density ratio does not change across a shock. The large region of density ratio near unity indicates that the majority of the flowfield in this configuration is jet core fluid, and again demonstrates very little mixing.

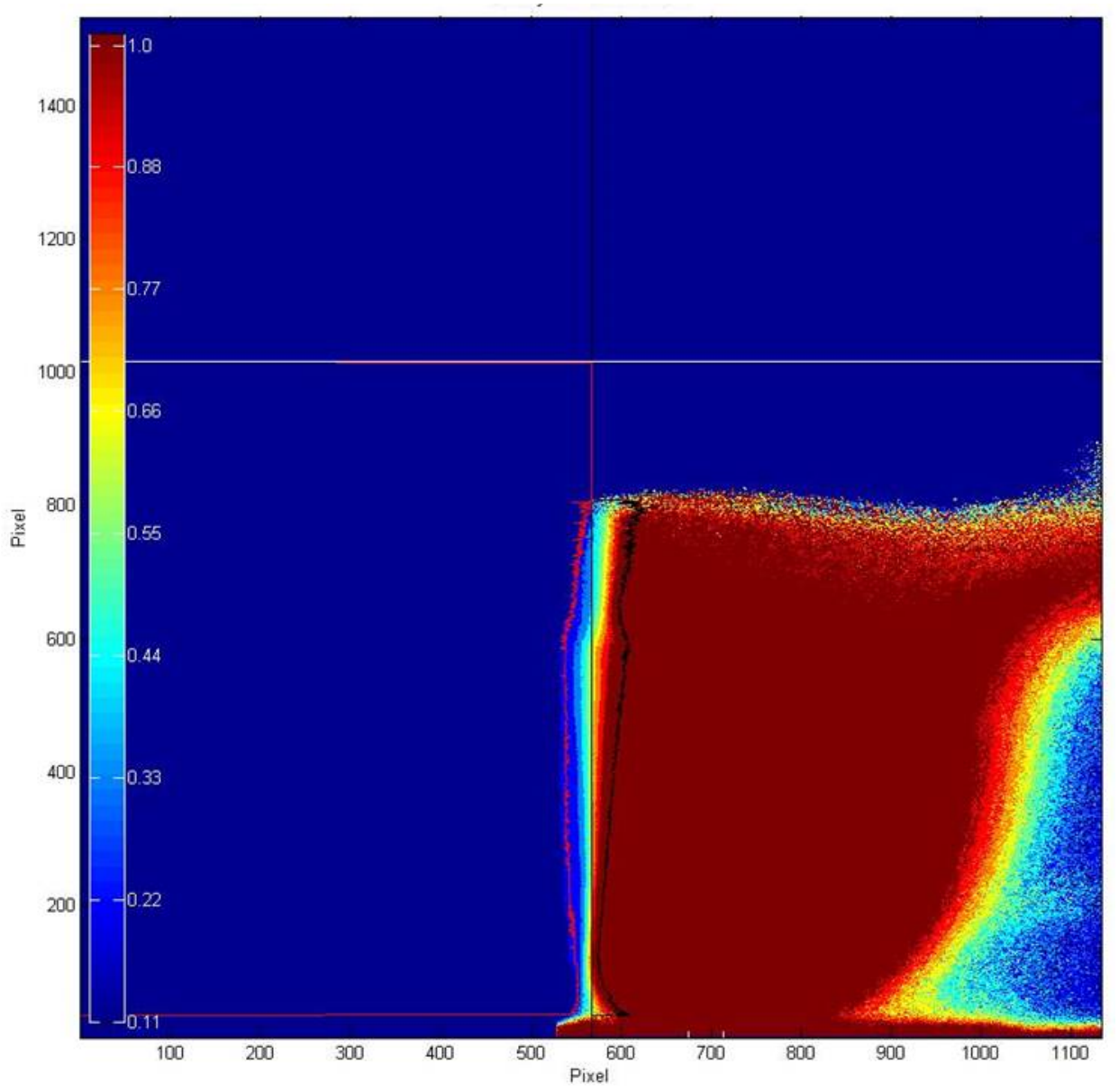

Figure 6.14: Density Ratio image - 2 Jet, 2.54" Standoff.

Figure 6.14, as with previous density ratio images, shows the line representing the integral $a$ boundary in Equation 6.1 in red, and the $b$ values from the same integral in black. Unlike the four jet impingement case, the mixing area could not be measured all the way to the impingement surface. Due to the loss of fluorescence signal, as is seen in Figure 6.13a, the density ratio could not be calculated and thus the mixing 
area could not be quantified all the way to the impingement surface. The initial neighboring jet interaction occurs between vertical pixels 75 and 100, 1.9 and 2.6 $\frac{x}{D}$ respectively, and shows an increase in mixing area from vertical pixel 100 to the location near the shock wave (as seen in Figure 6.13a) around vertical pixel 650 (16.6 $\left.\frac{x}{D}\right)$. The mixing area is very similar to the two jet free exhaust case, as well as the decrease in mixing area in the vicinity of vertical pixel $600\left(15.4 \frac{x}{D}\right)$.

The plot of the calculated mixing percentage, per Equation 6.4, is shown in Figure 6.15. Figure 6.15 represents the mixing percentage at each downstream (vertical) pixel.

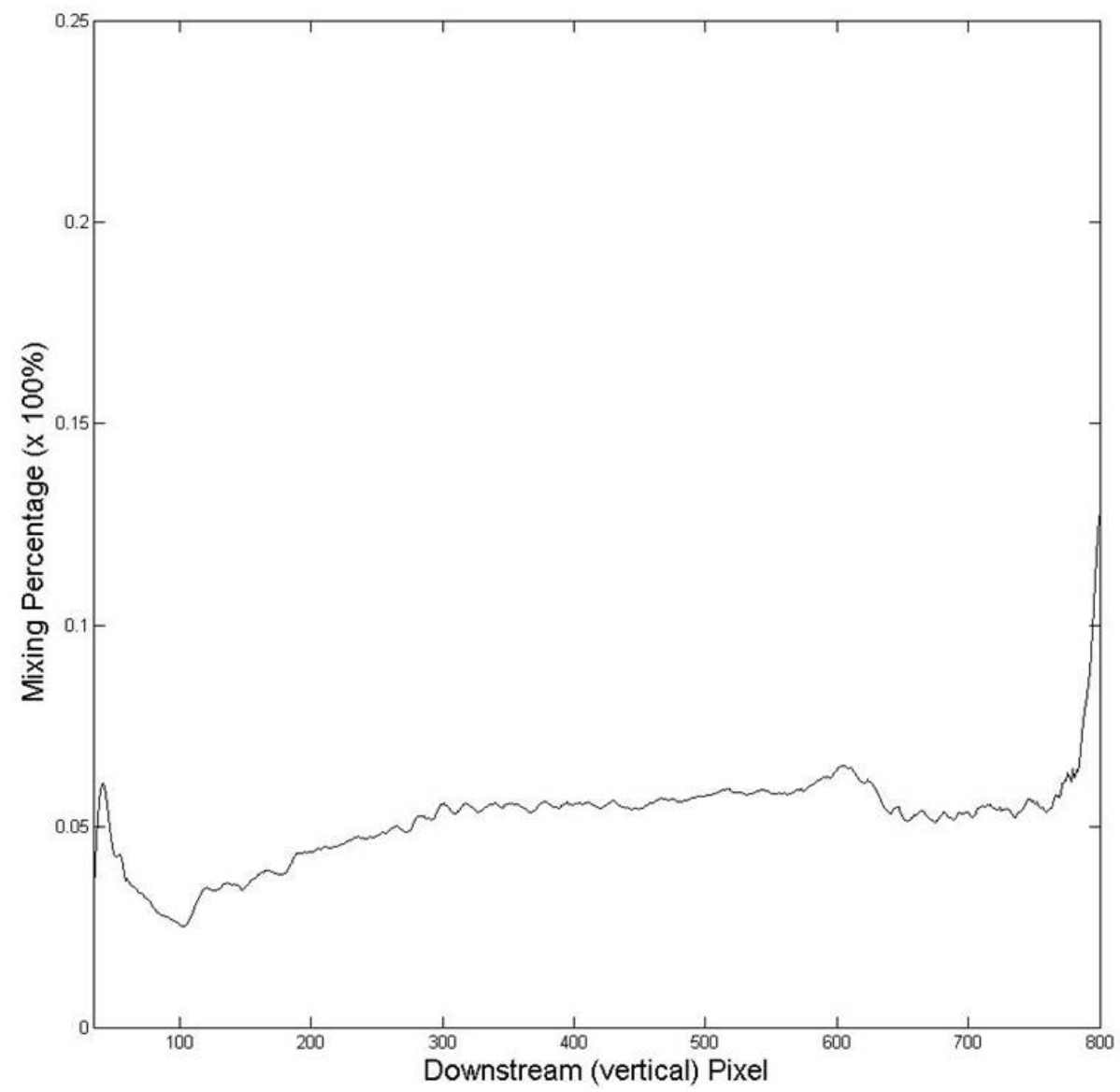

Figure 6.15: Mixing Percentage at each downstream pixel.

In Figure 6.15, the mixing percentage can be seen to remain nearly constant throughout the entire downstream fluid interaction. The total mixing percentage, as calcu- 
lated by Equation 6.5, for the two jet 2.54 inch impingement case is $5.2 \%$ at the most downstream pixel row. By comparing the maximum value for the impingement experiment to the equivalent location in the free exhaust experiment, some conclusions about the effect of the impingement surface on mixing area can be made. The value of the free jet mixing percentage at the downstream pixel equivalent of the impingement surface is $3.1 \%$, and for the impingement experiment that value is $5.2 \%$. This corresponds to an increase of approximately $70 \%$ to the neighboring jet interaction from the introduction of an impingement surface into the flowfield.

From the density ratio experiments and mixing area calculations, consistent conclusions can be made regarding the effect of the impingement surface on the mixing area interactions of neighboring jets. For both the four jet and two jet cases, the introduction of the impingement surface led to an approximately $70 \%$ increase in the total mixing percentage. Both the two jet and four jet neighboring interactions took on unique mixing characteristics that were consistent between the free and impingement experiments. The four jet experiments exhibited highly complex interactions that led to significant jet core fluid deflection, as well as very complex mixing along the entirety of the neighboring jet interaction. The neighboring jet interaction in the two jet case led to a small amount of jet core fluid deflection, but overall the mixing percentage was smaller than the four jet experiments. Between the free exhaust experiments, the two jet cases exhibited an approximately five times smaller total mixing percentage than the four jet configuration. For the impingement experiments, the two jet case exhibited an approximately six times smaller total mixing percentage than the four jet impingement configuration. By combining the flowfield geometry measurements from qualitative visualizations, and the quantitative measurements of the mixing area and density ratios, several unique conclusions can be made, as discussed in the next chapter. 


\section{Chapter 7}

\section{Conclusions and Recommendations}

Following the flight incident between the autonomous HTV spacecraft and the International Space Station, the University of Virginia's Aerospace Research Laboratory engaged in high speed fluids research in an effort to ascertain the root cause of the differences in the computational model and real-world fluid dynamics observed during the HTV maneuvers. Planar Laser Induced Iodine Fluorescence was utilized as the spectroscopic method with which to qualitatively and quantitatively investigate the complex hypersonic flowfield. Qualitative broadband visualizations were collected to visually classify each flowfield, as well as determine quantitative flowfield geometry measurements. Visualizations were collected for numerous configurations including varying the number of exhausting jets, varying the separation between neighboring jets, and for a variety of impingement standoff distances. Along with providing qualitative images of the flowfield interactions, quantitative flowfield density ratios were measured. The quantitative density ratio experiments were conducted for a smaller subset of experimental configurations. For the four jet configuration, density ratios were measured for the free exhaust and 3.52 inch $\left(36 \frac{x}{D}\right)$ standoff configurations. For the two jet flow configuration, density ratios for the free exhaust and 2.54 inch $\left(26 \frac{x}{D}\right)$ standoff configurations were measured. The combination of the qualitative images, geometry data, and density ratio data provides significant experimental insight into the complex jet-jet and jet-jet-surface interactions. 


\subsection{Visualization and Density Ratio}

The over-arching objective for this research is to investigate a hypersonic rarefied gas flowfield that is representative of the fluid flow encountered during an incident between a HTV autonomous spacecraft and the ISS. In order to fully investigate the incident, there were two primary research objectives. The first objective is to study the interaction of parallel Mach 5 under-expanded jets. This involved experiments where Mach 5 jets are allowed to exhaust into a quiescent atmosphere. The goal of these experiments were to determine the effect of center-to-center spacing on jet-jet interactions, as well as quantify the mixing resulting from the jet-jet interactions.

The second research objective was to determine the impact of the introduction of an impingement surface on the jet-jet interactions. By introducing an impingement surface into the flowfield at a number of standoff distances, this investigation offers insight into the effect of the impingement surface on the mixing interaction, as well as changes to flowfield geometry. The introduction of an impingement surface into the flowfield also allowed for the characterization of the impact of the impingement surface on neighboring jet interaction and mixing.

In order to achieve these two main objectives, two experimental approaches were utilized during this research. First, broadband Planar Laser Induced Iodine Fluo-

rescence (PLIIF) was used for flowfield visualization. This experimental approach provided the data necessary to determine the effect of center-to-center spacing on jet-jet and jet-jet-impingement surface interactions. Secondly, PLIIF quantitative density ratio imaging was employed to quantify the mixing resulting from the jet-jet and jet-jet-impingement surface interactions. By focusing on these two research objectives, a thorough investigation into the jet-jet interactions, and the contribution to mixing from the introduction of an impingement surface, was completed. 


\subsection{Research Objective 1}

From the qualitative broadband visualization images, some initial insight into the nature of the neighboring jet interaction was available. As outlined in Chapter 4 . three nozzle disks were selected to vary the center-to-center spacing of the Mach 5 under-expanded jets. Disk 1, with center-to-center spacing of $15.2 \mathrm{~mm}$, exhibited significant neighboring jet interaction with four jets exhausting freely. The jet core flow is significantly deflected, and a normal shock forms at $26.8 \frac{x}{D}$. The neighboring jet interaction occurs at $2.7 \frac{x}{D}$ downstream of the jet exit. Disk 2, with centerto-center spacing of $22.2 \mathrm{~mm}$, with four jets exhausting exhibited no neighboring jet interaction. The third disk, with a center-to-center spacing of $57.1 \mathrm{~mm}$, also exhibited no jet-jet interactions in the free exhaust configuration. In addition to experiments using four jets, experiments using only two jets exhausting were conducted based on current operational recommendations for the HTV spacecraft. Utilizing Disk 1, with only two jets exhausting, neighboring jet interactions were observed to a smaller degree than those observed with four jets exhausting. There was no significant core flow deflection, and the interaction region appeared to be smaller. The interaction of the neighboring jets was observed to occur at $2.9 \frac{x}{D}$ downstream of the nozzle exit.

Once it was observed that both two and four jet flow configurations exhibited neighboring jet interactions, quantitative density ratio experiments were conducted to determine the nature of the jet-jet interactions. By conducting quantitative density ratio measurements, as outlined in Chapter 3 , a thorough investigation of the jet-jet interactions could be made. Using the data conducted in the density ratio experiments to calculate the total mixing percentage, as covered in Chapter 6, a comparison of the impact of the number of jets on neighboring jet interaction could be made. Both free exhaust density ratio experiments used the Disk 1 center-to-center spacing. The

Disk 1, four jet free exhaust configuration yielded a total mixing percentage of 19.7\%, 
which indicates that approximately $20 \%$ of the fluid from a single jet is involved in neighboring jet interactions and mixing. For the two jet experiment, utilizing Disk 1, the total mixing percentage was calculated to be 3.7\%. By comparing these values, a five fold increase in neighboring jet interaction is observed with the introduction of the out-of-plane jets.

From the investigation into this research objective two primary assertions can be made. First, by increasing the jet spacing the neighboring jet interaction decreases, as expected. Second, the removal of two exhausting jets led to a 5 times smaller mixing percentage, which means mixing was enhanced by the two additional jets. The core flow in the two jet case was not deflected appreciably, and the interaction region was much smaller than when the out-of-plane jets contributed to the flowfield. A summary of all results used for the neighboring jet interaction comparison can be found in Appendix A. From these results, comparisons with results from the second research objective provide a complete picture of the complex flowfield encountered during the HTV maneuver.

\subsection{Research Objective 2}

For the second research objective, the interaction location and maximum flowfield width from the visualization images provides some very interesting results with an impingement surface. For Disk 1, the four jet free exhaust experiment interacted at 2.7 $\frac{x}{D}$, while the impingement experiments interacted between 2.8 and $2.9 \frac{x}{D}$. A similar outcome was observed for the Disk 1 two jet experiments. The two jet free exhaust configuration interacted at $2.4 \frac{x}{D}$ downstream, while the impingement experiments interacted between 2.0 and $2.5 \frac{x}{D}$. From these results, it can be observed that the introduction of the impingement surface did not appreciably impact the downstream location of the initial neighboring jet interaction. An interesting observation was that 
the Disk 2 four jet impingement experiment, with an impingement standoff distance of 2.1875 inches, did exhibit neighboring jet interactions. The minor deflection of the core flow was due to the proximity of the free pressure boundary created by the compressive interaction with the impingement surface. The impingement experiment for the widest jet spacing, Disk 3, showed no neighboring jet interactions. From the same visualization images used to investigate the initial interaction location, some observations concerning the flowfield width can be made.

In order to continue to characterize the impact of the impingement surface on neighboring jet interactions, a comparison of maximum flowfield width and normal shock location is needed. For Disk 1, the 4 jet free exhaust flowfield has a maximum flowfield width of $16.3 \frac{w}{D}$, while the impingement experiments yielded maximum widths between 16.0 and $16.8 \frac{w}{D}$. Using the same disk, the two jet free exhaust configuration achieved a maximum flowfield width of $19.7 \frac{w}{D}$. The impingement experiments for the two jet configuration achieved maximum widths ranging between 18.7 and $20.3 \frac{w}{D}$. For each flow configuration, either four or two jet, it can be seen that the introduction of the impingement surface did not appreciably impact the maximum flowfield width. The introduction in the impingement surface did yield variation in the configuration of the normal shock in the flow. The four jet experiments formed a single normal shock over the interacting region in both the free exhaust and impingement experiments. Conversely, the two jet experiments yielded a small normal shock over the interacting region in the free exhaust experiment, but separated into two normal shocks above the core fluid flow in the impingement configurations. It can also be seen in Table A.1, that the reduction in standoff distance corresponds to a reduction in downstream location of the normal shock. By measuring the shock locations, a relation between impingement standoff distance and normal shock formation was found. The normal shock in impingement cases was found to occur, on average, at $60 \%$ of the impingement standoff distance. 
Utilizing the quantitative density ratio measurements, along with the calculation of mixing percentage for both the free exhaust and impingement configurations, a quantitative determination of the effect of the impingement surface on the jet-jet interactions is possible. For Disk 1, the four jet free exhaust experiment yielded a total mixing percentage of $19.7 \%$. The Disk 1, four jet 3.52 inch impingement standoff distance experiment exhibited a total mixing percentage of $29.7 \%$. The total mixing percentage at the downstream pixel location of the impingement surface is calculated for the free exhaust experiment, a total mixing percentage of $17.0 \%$ is found. If the total mixing percentage at the downstream location of the impingement surface is compared between the free exhaust and impingement case, an approximately $70 \%$ increase in total mixing percentage due to the introduction of the impingement surface is observed.

For the two jet free exhaust experiment, using Disk 1 spacing, a total mixing percentage of $3.7 \%$ is found, demonstrating very little mixing. By introducing an impingement surface at a standoff distance of 2.54 inches, the total mixing percentage becomes $5.2 \%$. Again, by calculating the total mixing percentage at the impingement surface in the free exhaust experiment, a value of $3.1 \%$ is found. By comparing this value to the mixing percentage in the impingement case, an approximately $70 \%$ increase in mixing percentage is observed. For both the four jet and two jet flow configurations, the introduction of the impingement surface to the flow yielded an approximately $70 \%$ increase in total mixing percentage, which means there was an approximately $70 \%$ increase to the neighboring jet interactions. Combining the observations from the first research objective with the results found under the second research objective, a full understanding of the impact of neighboring jet interactions and jet-jet-impingement surface interactions is achieved.

The interactions observed utilizing the most geometrically similar jet center-to- 
center spacing, Disk 1, shows that a decrease in jet spacing results in an increase in jet-jet interactions. With the introduction of an impingement surface into the flowfield, the jet-jet interactions are increased, and can even result in neighboring jet interaction that is not present during free exhaust flow. This research provides important insight into the complex fluid dynamics of the hypersonic under-expanded neighboring jet interactions, as well as the interactions with an impingement surface. Thorough insight into the fluid dynamics involved in the flight incident will lead to significant improvements in computational modeling of these complex fluid flows, as well as changes to operational flight procedures around the ISS.

\subsection{Impact of this Research}

The research presented here has demonstrated the extremely complex nature of hypersonic jet-jet interactions. This research is important to the field of hypersonic rarefied gas dynamics research for a number of reasons. First, this research ultimately provides sets of experimental data to the NASA Johnson Space Center. As part of the real-world impact this research will have, the data will be used to refine the DAC predictive model for Mach 5 rarefied jet-jet and jet-jet-surface interactions. The results from the refined model, as well as from flowfield data collected during this research, will result in modifications to real-world flight procedures for all spacecraft operating in the vicinity of the International Space Station. Additionally, the refined DAC model will be more robust in its predictions when applied to a variety of other hypersonic flow conditions.

Secondly, the improved predictive models will have an impact on better understanding the complex flows encountered during retro-propulsion for planetary entry and deceleration. By increasing comprehension of neighboring jet interactions, it will allow spacecraft designers to optimize maneuvering and deceleration jet locations. 
This will in turn allow for optimized control of larger payloads during planetary entry and atmospheric deceleration. This work further contributes to previous research efforts into the understanding of propulsive deceleration, which was conducted as part of an investigation into landing large payloads on Mars $\$$.

Thirdly, the results from the impact of impingement surface interaction on neighboring jet fluid mixing not only applies to orbiting spacecraft, but also to spacecraft landing on celestial bodies with a low density atmosphere, or even no atmosphere. By understanding how under-expanded hypersonic neighboring jets interact, optimized designs can be developed for increased control and performance of spacecraft landing on celestial bodies. Similar to the second impact of this research, the knowledge gained from these experiments will facilitate the design of higher performance spacecraft, especially performance optimization during the landing phase. As a result, these designs will allow for larger payloads and more robust control of these larger spacecraft during planetary landings. By gaining a fuller understanding of the complex dynamics of hypersonic neighboring jet interactions, as well as the nature of the interaction with impingement surfaces, an improvement in spacecraft propulsion design is possible. This could facilitate the production of spacecraft capable of safely traveling, entering the atmosphere (if present), and landing on a planetary body with a large payload capable of supporting the current planned exploration missions, namely the manned exploration of Mars.

\subsection{Recommendations for Future Work}

Continued research into the interaction of hypersonic under-expanded jet flowfields would result in a more thorough computational and experimental understanding of the nature of rarefied fluid dynamics. Further investigation into the flowfield geometry from parallel under-expanded jets, namely the maximum flowfield width, would 
provide significant additional information necessary for application to industry engine design efforts. This investigation could be achieved by matching the $P_{\text {ratio }}$ values between the two and four jet flow configurations. For the laboratory equipment outlined in Chapter 4 of this work, that could be achieved by modifications to the vacuum pumping system or to the liquid nitrogen cooling loop.

A primary effort of future research should be the application of the single-frequency etalon scanning technique. This method was outlined briefly in Chapter 3 and used on other supersonic flowfields in previous research. This technique would allow for the collection of quantitative flowfield thermodynamic and velocity data in any of the experimental configurations outlined in this work. This additional research would provide invaluable insight into the complexity of the interactions which were not obtained under the purview of this research. In order to collect these measurements at the Aerospace Research Laboratory, equipment in the form of a new argon-ion laser and a re-designed etalon would be necessary.

Finally, while NASA Johnson will utilize the test data to refine their computational model, applications of newer hybrid and DSMC modeling techniques would provide ample grounds for future research. The application of hybrid codes like LeMANS 50151 could prove to more accurately model the complex flow, when compared to traditional DSMC models. Based on previous research, the application of a hybrid code, specifically LeMANS, has resulted in excellent agreement with experimental data. Traditional DSMC codes such as SPARTA, SMILE, and dsmcFoam would also benefit from being applied to this research. By using another traditional DSMC code to model the experiments conducted in this research, the predictive algorithms in these codes could be modified to make them more accurate and applicable to extremely complex rarefied flows. By applying these analysis codes, significant evolution in the algorithms and predictive capability of the codes will be realized. 


\section{Appendix A}

\section{Data Summary Table}

This appendix contains summary tables for the experimental data presented in Chapters 5, 6, and 7] of this research.

\begin{tabular}{|c|c|c|c|}
\hline Experimental Configuration & Interaction Location $\left(\frac{x}{D}\right)$ & Maximum Flowfield Width $\left(\frac{w}{D}\right)$ & Shock Location $\left(\frac{x}{D}\right)$ \\
\hline Disk 1 - 4 jet, free exhaust & 2.7 & 16.3 & 26.8 \\
\hline Disk 1 - 4 jet, 3.62" standoff & 2.8 & 16.8 & 22.4 \\
\hline Disk 1 - 4 jet, 2.875" standoff & 2.9 & 16.4 & 19.5 \\
\hline Disk 1 - 4 jets, $2.1875 "$ standoff & 2.9 & 16.0 & 16.8 \\
\hline Disk 1 - 2 jet, free exhaust & 2.4 & 19.7 & 27.2 \\
\hline Disk 1 - 2 jet, 3.62" standoff & 2.4 & 18.7 & 24.7 \\
\hline Disk 1 - 2 jet, 2.875" standoff & 2.0 & 19.4 & 15.5 \\
\hline Disk 1 - 2 jet, 2.1875" standoff & 2.1 & 20.3 & 14.1 \\
\hline Disk 2 - 4 jet, free exhaust & NONE & 6.9 & NO SHOCK \\
\hline Disk 2 - 4 jet, 2.1875" standoff & UNIDENTIFIED & 16.0 & 13.2 \\
\hline Disk $3-4$ jet, free exhaust & NONE & 7.3 & NO SHOCK \\
\hline Disk $3-4$ jet, 2.1875" standoff & NONE & 7.2 & 14.4 \\
\hline
\end{tabular}

Table A.1: Pertinent geometry measurements.

\begin{tabular}{|c|c|c|}
\hline Experimental Configuration & Total Mixing Percentage (max, \%) & Total Mixing Percentage at Impingement Location (\%) \\
\hline \hline 4 jet, free exhaust & 19.7 & 17 \\
\hline 4 jet, 3.52" standoff & 29.7 & 29.7 \\
\hline \hline 2 jet, free exhaust & 3.7 & 3.1 \\
\hline 2 jet, 2.54" standoff & 5.2 & 5.2 \\
\hline
\end{tabular}

Table A.2: Mixing Percentage Comparison. 


\section{ApPendix B}

\section{UNCERTAINTY ANALYSIS}

\section{B.1 Uncertainty Analysis for Broadband Techniques}

Since the flowfield geometric measurements were calculated based on broadband fluorescence images, there are two primary sources for uncertainty in the measurement. The first source of uncertainty comes from the alignment of the laser sheet with the center of the jet exits, and the second is introduced by the finite width of shock and boundary region fluorescence signals.

The approximation of the uncertainty arising from the laser sheet alignment stems from two sources. Linear misalignment refers to any misalignment that occurs when the laser sheet is shifted horizontally off the centerline of the two jets that are bisected during the measurement. An angular misalignment occurs when the laser sheet is not perpendicular to the jet exit, therefore not bisecting the flowfield vertically. The linear misalignment would indicate that the flowfield florescence captured by the CCD would contain information the did not accurately reflect the centerline flow characteristics in each jet core. Based on the optical configuration used in these experiments, a laser sheet thickness of approximately 0.5 millimeters is present at the jet exits. This thickness is $\frac{1}{5}$ of the diameter of the jet exit, making the alignment uncertainty at most $\frac{2}{5}$ of a jet exit diameter. Since the size of the laser sheet is small, and the optical alignment procedures robust, the linear misalignment is minimal and not expected to be a major source of uncertainty. The alignment of the model with 
respect to the CCD camera is also confirmed before the start of every experiment, so an introduction of uncertainty due to model misalignment is considered negligible. With respect to the angular misalignment, the angle of both the optical train and the tunnel internal mirror were measured and set accurately prior to each experimental run. The accuracy of the tools used to align these apparati has an accuracy of $0.25^{\circ}$. If both the optical train and internal mirror are assumed to be out of alignment with one another, a total error in the angle-of-attack of the laser sheet would be approximately $1^{\circ}$. With a total possible uncertainty of $1^{\circ}$ in the laser sheet angle-ofattack, significant deviation in Doppler bias between experiments will not be present. This means that the fluorescence signal will be highly repeatable, leading to minimal uncertainty due to laser sheet alignment.

The uncertainty in measurements of flow geometry arising from analyzing broadband images is not negligible. In order to account for error in geometry measurements for each flowfield, the size of the region defining the flow feature of interest can be used as a gauge for the range of variability in the measurement. Because flow features, such as a normal shock or flow boundary, contain significant changes in pressure, the fluorescence signal will correspondingly vary across the feature. This variation of fluorescence signal is used to define the flow feature, and presents a finite range of fluorescence signal that constitutes the flow feature. In the case of measuring the flow width, the size of the jet flow boundary fluorescence signal will determine the uncertainty in the width measurement. This means that from the fluorescence signal that indicates the inner edge (on the jet core flow side) of the jet boundary to the free pressure boundary edge, a finite width for the flow feature can be determined. On average, the jet flow boundary on the non-neighboring-jet side of the flow is found to be 20 pixels wide. With an uncertainty in flowfield width of 20 pixels, the relative uncertainty in flowfield width for the four jet experiments is $3.1 \%$. For the two jet experimental configuration, the maximum flowfield width uncertainty is $2.6 \%$ of 
maximum flowfield width.

Similar to the fluorescence signal width for the jet boundary, the normal shock represents a rise in pressure and collisional quenching of the iodine molecules. Since this rise in pressure occurs over a finite distance in the flowfield, the fluorescence signal that represents the normal shock also has a finite width. This finite width in fluorescence signal represents a small region in which the normal shock is present. For the normal shock measurements, the vertical width of the shock region is an average of 30 pixels tall. With an uncertainty in normal shock downstream location of 30 pixels, the relative uncertainty for the downstream location of the normal shock is $4.2 \%$.

Since laser sheet alignment is considered a negligible source of uncertainty, the uncertainty for flowfield geometry measurements is approximately $\pm 1.5 \%$ for the maximum jet width measurements, and $\pm 2 \%$ for the normal shock downstream location measurement. Since the downstream location of the neighboring jet interaction was determined based on the same criteria in fluorescence signal used to determine the jet plume boundary location, an error of $\pm 1.5 \%$ is also applied to those measurements.

\section{B.2 Uncertainty Analysis for Density Ratio}

In this section of the Appendix, the analysis of the uncertainty in the density ratio measurements will be presented. The experimental uncertainty associated with the data collection and the systematic error associated with the operation of the experiment will be addressed. Following an error analysis method similar to previous works ${ }^{48}$ an equation for the error in density ratio measurements can be determined.

Referring back to the equations presented in Chapter 3 , the density ratio equation 
can be written as:

$$
\text { Density Ratio }=\frac{n_{\text {SingleJet }}}{n_{\text {Total }}}=A \frac{S_{f \text { SingleJet }}}{S_{\text {fTotal }}}
$$

where the left side of the equation is the number density ratio, $A$ is the density ratio determined where the ratio of fluorescence signals is know, and the $S_{f}$ variables represent the fluorescence signal collected for either the single jet seeded with iodine, or the entire flowfield seeded with iodine. By taking the logarithmic derivative of Equation B.1, an equation for experimental uncertainty in the density ratio measurements can be written as:

$$
\frac{\Delta \text { Density Ratio }}{\text { Density Ratio }}=\frac{\Delta A}{A}+\frac{\Delta S_{f \text { SingleJet }}}{S_{f \text { SingleJet }}}-\frac{\Delta S_{f \text { Total }}}{S_{f \text { Total }}}
$$

where each term in Equation B.2 represents the uncertainty associated with the measurement of each individual parameter in the density ratio calculation. Before performing the density ratio calculation in Equation B.1, the background signal is subtracted from both the single and fully seeded fluorescence data, though the background subtraction technique cannot fully eliminate the scattering effect of the laser sheet interacting with the model surface. However, after a short distance downstream from the model surface, the background signal is almost completely eliminated with this technique. Because of the nature of the background subtraction, the constant background signal is not taken into account in the uncertainty analysis. This means that the uncertainty in $S$ is attributed to the photon statistical noise ${ }^{61}$ plus a residual background value given by:

$$
\Delta S=\sqrt{S}+k S
$$

where $\mathrm{k}$ is a constant used to account for the residual background. By substituting 
Equation B.3 into Equation B.2, the resulting equation can be written as:

$$
\frac{\Delta \text { Density Ratio }}{\text { Density Ratio }}=\frac{\Delta A}{A}+\left[\frac{1}{\sqrt{S_{\text {fSingleJet }}}}+k_{\text {SingleJet }}\right]-\left[\frac{1}{\sqrt{S_{\text {fTotal }}}}+k_{\text {Total }}\right]
$$

Re-writing Equation B.4 in standard deviation form yields:

$$
\begin{array}{r}
\sigma\left(\frac{\Delta \text { Density Ratio }}{\text { Density Ratio }}\right)= \\
\qquad \sqrt{\left(\frac{\Delta A}{A}\right)^{2}+\left[\frac{1}{S_{\text {fSingleJet }}}+\left(k_{\text {SingleJet }}\right)^{2}\right]-\left[\frac{1}{S_{\text {fTotal }}}+\left(k_{\text {Total }}\right)^{2}\right]}
\end{array}
$$

A typical value for the fluorescence photo-electron count at a single pixel on the detector, in the applicable portion of the flowfield, is measured to be 30750. After background subtraction from the image, the residual background is typically less than $0.5 \%$ of the maximum signal level. The error in determining the constant ratio $\frac{\Delta A}{A}$ is estimated to be around 1\%. Using these values and Equation B.5, the experimental uncertainty for the density ratio measurements is $1.2 \%$.

Systematic errors are difficult to evaluate for a complex experiment of this nature and there is no rigorous, highly developed method to account for systematic error in the overall uncertainty analysis $\frac{62}{}$. Because of this difficulty, a conservative estimate for systematic uncertainty due to change in plenum total conditions during the density ratio experiments will be made and added linearly to the experimental uncertainty outlined above. Both the single jet plenum pressure and the three jet plenum pressure are independently controlled with accurate regulators and gauges, as outlined in Chapter 4. While the plenum pressures are accurately controlled for the experiment, the total temperature for the iodine seeded nitrogen is not specifically controlled. Heating of the iodine mixing vessel is in place to create a constant total temperature for the iodine mixing, but during density ratio experiments ni- 
trogen bypasses the mixing vessel in order to only seed a single jet. This bypass nitrogen that proceeds to the three jet plenum does not have a temperature control scheme similar to the mixing vessel. This is a very complex situation to account for, but based on typical operating temperatures for the nitrogen bank compared to the temperature controlled mixing vessel, a difference of approximately $1 \%$ of total temperature could be expected. The sensitivity of the fluorescence signal to temperature varies exponentially according to the Clausius-Claperon equation for vapor pressure. While variation in total temperature will effect the fluorescence signal of the iodine, this is accounted for in the constant in the density ratio calculation. By the nature of the density ratio calculation, specifically by taking the ratio of two fluorescence images with approximately identical fluid operating temperatures, any variation in temperature during the experiment is not a significant source of error. Therefore, a total uncertainty of $1.2 \%$ is calculated, based on the experimental uncertainty being the primary contributor to overall density ratio uncertainty. Based on this value, the uncertainty of the density ratio experiment is considered to be $\pm 0.6 \%$. 


\section{ApPENDix C \\ LATVALA \& ANDERSON Jet BOUndARY PREDICTION}

This appendix contains the Matlab code for the script used during the initial design phase of this research. It was used as a secondary check to ensure neighboring jet interaction when selecting the smallest center-to-center measurement. It is based on the Method of Characteristics approach, and its use is detailed in Chapter 4.

$\%$ Script to calculate the radius of a an underexpanded supersonic jet $\%$ plume. Based on a method proposed by Latvala \& Anderson proposed in 1959. \% Method closely approximates the method of characteristics solution. function Latvala_jet_radius()

clear all; clc

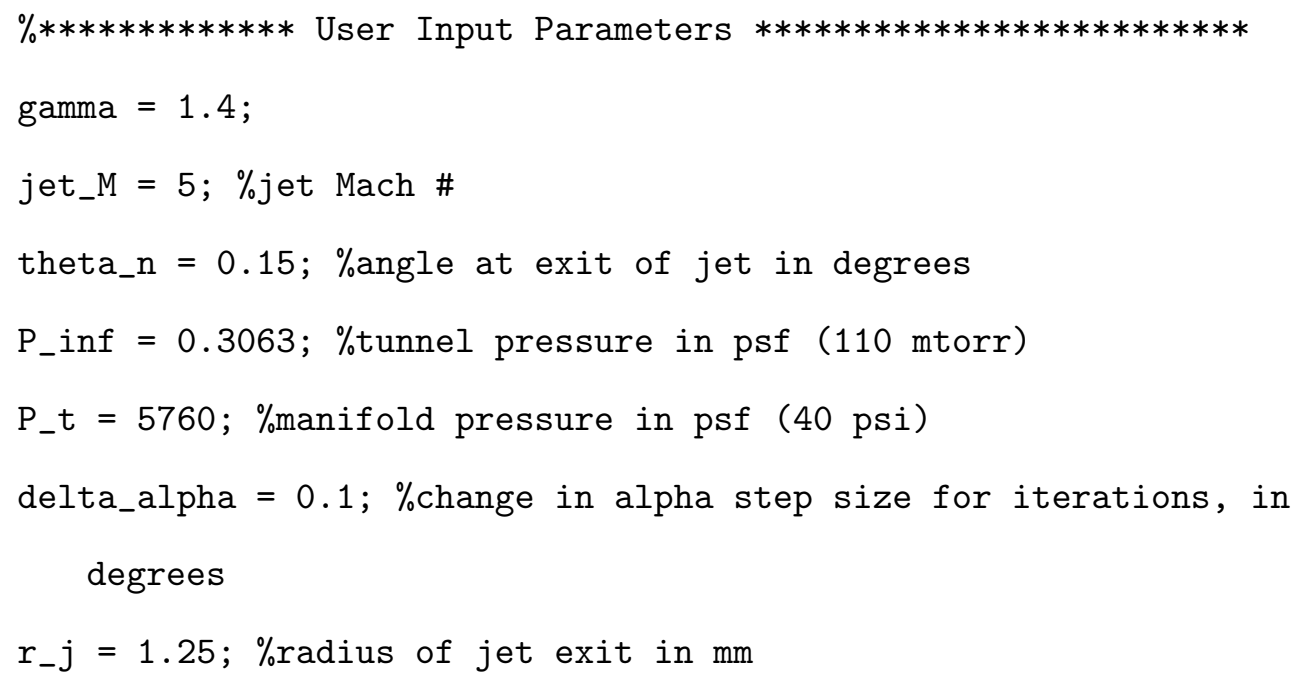


$q=300 ; \%$ matrix size and number of loop iterations

$\% * * * * * * * * * * * * * * * * * * * * * * * * * * * * * * * * * * * * * * * * * * * * * * * * * * * * * * * * * * * * * * *)$

$\begin{array}{lllllll}\% & 1 & 2 & 3 & 4 & 5 & 6\end{array}$

$\%$ init solution matrix; contains ( $n u, M, A / A *$, alpha, $r / r_{-} j$, delta_X/r_j )

solution $=\operatorname{zeros}(q, 6)$;

\%Calculate P-M angle for jet exit

$n u_{-} j=\operatorname{sqrt}(($ gamma +1$) /($ gamma -1$)) * \operatorname{atand}(\operatorname{sqrt}((($ gamma -1$) /($ gamma +1$)) *($ jet_M^2-1)))-atand (sqrt (jet_M^2-1));

$\% * * * * * * * * * * * * *$ Point 1 Calculations

$\%$ Calculate other point to determine info for Point 1 (jet exit start \%point). Solve for Mach\#, P-M angle, and Area Ratio at point 1 $\%$ (effectively the jet exit). delta_X for point 1 is zero b/c it is $\%$ located at jet exit.

P_ratio_inftot $=P_{-}$inf $/ P_{-} t$;

syms $M$;

tmp_M $=\operatorname{solve}\left(\left(1+(\text { gamma-1) }) / 2 * M^{\wedge} 2\right)^{\wedge}(-\right.$ gamma $/($ gamma -1$))==$

P_ratio_inftot, M);

$\operatorname{solution}(1,2)=$ tmp_M(1);

$\% \mathrm{P}-\mathrm{M}$ angle

solution $(1,1)=\operatorname{sqrt}(($ gamma +1$) /($ gamma -1$)) * \operatorname{atand}(\operatorname{sqrt}((($ gamma -1$) /($ gamma

$\left.\left.+1)) *\left(\operatorname{solution}(1,2)^{\wedge} 2-1\right)\right)\right)$-atand (sqrt (solution $\left.\left.(1,2)^{\wedge} 2-1\right)\right)$;

\%Area Ratio based on Mach number above

solution $(1,3)=((\text { gamma }+1) / 2)^{-}(-($gamma +1$) /(2 *($ gamma -1$))) *((1+($ gamma -1$)$

$/ 2 *$ solution $\left.\left.(1,2)^{\wedge} 2\right)^{\wedge}((\operatorname{gamma}+1) /(2 *(\operatorname{gamma}-1)))\right) / \operatorname{solution}(1,2)$;

\%Calculate jet boundary tangent angle (alpha in degrees)

solution $(1,4)=$ solution $(1,1)-n u_{-} j+$ theta_n; 


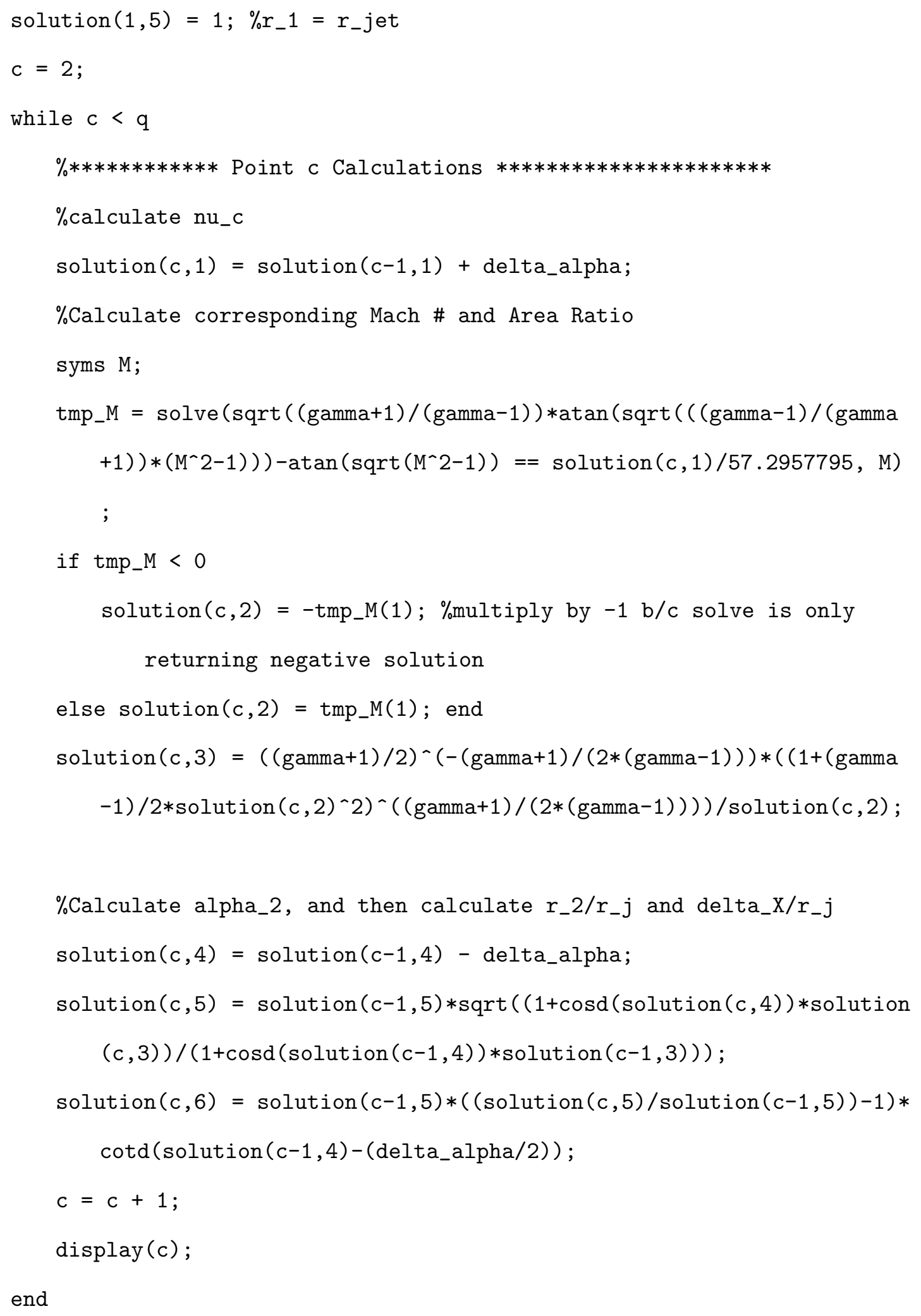


save('Latvala_solution.mat', 'solution'); \%save the solution matrix out of the script workspace

csvwrite('Latvala_solution.csv', solution);

end 


\section{Appendix D}

\section{Iodine Model Matlab Code}

This appendix contains the (slightly modified) iodine model that has been used repeatedly in PLIIF research conducted in this lab, and has proven to very accurately model the iodine spectrum. It has been modified specifically for the analysis of the hyperfine iodine spectrum resolving in hypersonic jet core fluorescence signals, as presented in this research. The only modifications that were made for the research dealt with cleaning up the code visually and some small optimization changes, none of which effected the accuracy of the code. The iodine model consists of two Matlab scripts: IodineModelSpectrumAnalsysis.m (iodine hyperfine model) and Humdev2.m (solution method for solving the Voigt function convolution numerically).

\section{IodineModelSpectrumAnalysis.m:}

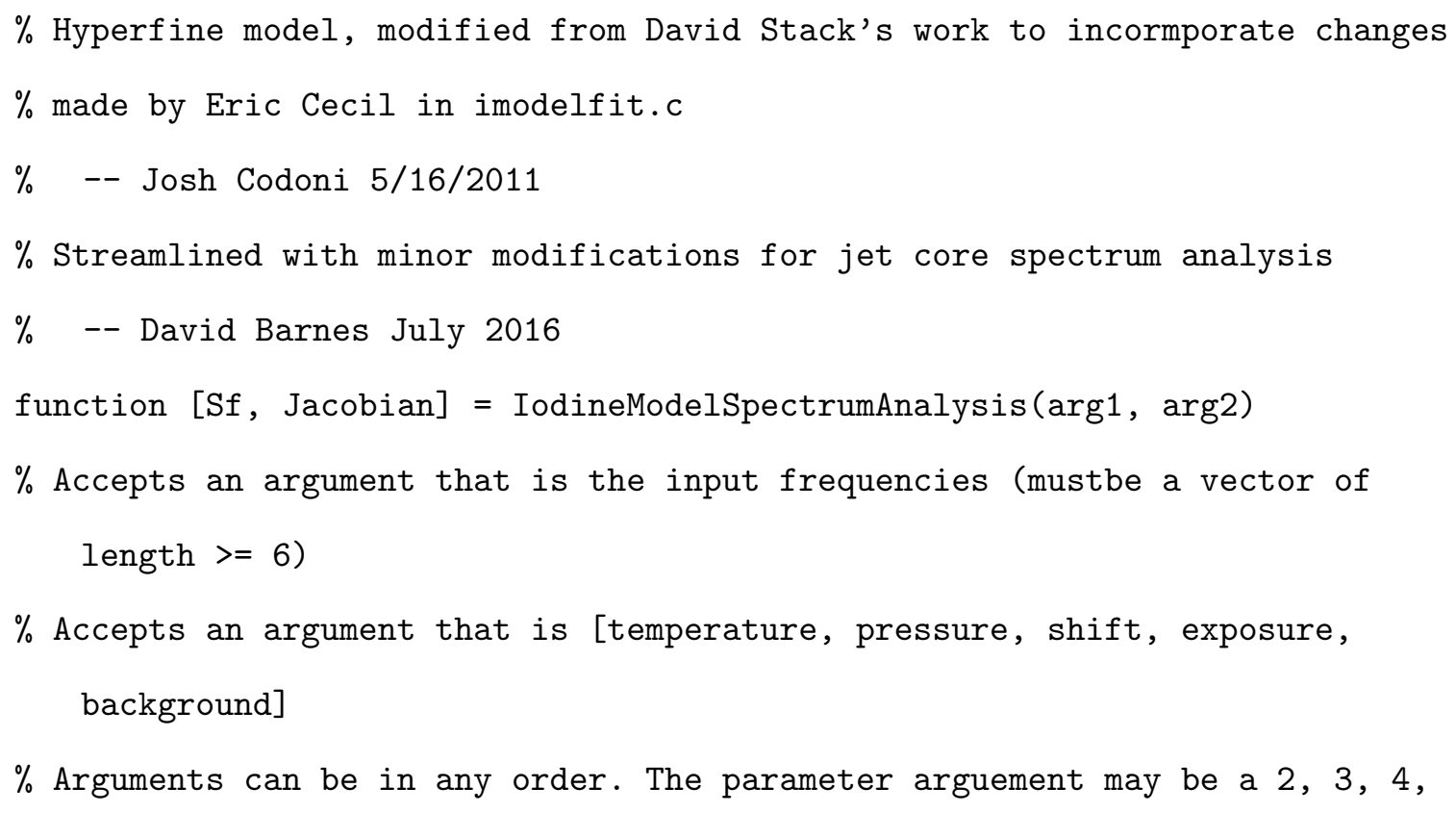


or 5 vector.

$\%$ Kelvin, Atmospheres, GHz; the Sf has no units

$\%$ Sf: a column vector of Fluorescence

\% Jacobian: matrix dSf_dparam, where param = [temp, pres, shift, expo, bgrnd]

if length $(\arg 1)<=5 \%$ called as (param, freq)

$\operatorname{nmax}=$ length $(\arg 2) ;$

temp $=\arg 1(1)$;

pres $=\arg 1(2) ;$

if length(arg1) $>2$, shift $=\arg 1(3) ;$ else shift $=0$; end;

Frequency $=\operatorname{reshape}(\arg 2,[\operatorname{nmax}, 1])-\operatorname{shift} ;$

else $\%$ called as (freq, param)

$\operatorname{nmax}=$ length $(\arg 1)$;

temp $=\arg 2(1)$

pres $=\arg 2(2)$;

if length $(\arg 2)>2$, shift $=\arg 2(3)$; else shift $=0$; end;

Frequency $=\operatorname{reshape}(\arg 1,[\operatorname{nmax}, 1])-$ shift;

end

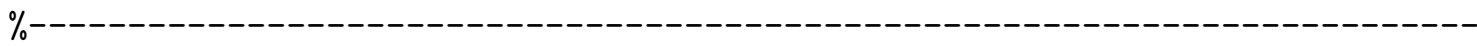

$\%$ CONSTANTS USED

$\mathrm{Cq}=1.5 ; \% \mathrm{Cq} / \mathrm{A} 21$ Hartfield Value $\mathrm{K}-0.5 / \mathrm{kPa}--$ Donohue used 2.1 as did Stack

$\mathrm{Cq}=\mathrm{Cq} \cdot * 101 \cdot 3 ; \% \mathrm{Cq} \rightarrow \mathrm{atm}$

$\mathrm{Cb}=4.1962 . * 101.3 ; \%$ ?? 4.4 ?? GHz K - 0.7 / KPa Donohue value (from Cecil

)

$\%$ dhfw not used by Eric, he includes derivitives of Voigt and dnuL / dnuG

$\% \operatorname{dhfw}=.0075$

$\%$ Cimpact $=.39$;

$\%$ Cimpact $=$ Cimpact.$* 101.3 ; \%$ for atm 
\% Einstein spontaneous emission coef vs V", normalized by the same for V" = \% 43 (i.e. P13/R15). This is Jim Donohue's 4th order polynomial (?1) fit to $\%$ fig. 8 of paper by Capelle \& Broida. Considering the scatter of data, $\%$ this all is questionable. Needless to say, his a's are not meaningful to $\% 13$ significant figures -- so only using float (approx 7 sig figs). If the $\%$ quenching is tiny, this won't figure into things.

$\%$ - Eric Cecil

\%instein spontaneous emmision coef vs V" , approximate curve from Lehman $\mathrm{a} 0=4.381977209869 \mathrm{E}+00 ;$

$\mathrm{a} 1=-1.587067053876 \mathrm{E}-01 ;$

$\mathrm{a} 2=2.840871548044 \mathrm{E}-03$;

$\mathrm{a} 3=-2.832453672502 \mathrm{E}-05 ;$

$\mathrm{a} 4=1.280451088469 \mathrm{E}-07$;

$\%$ These commands read in the transition data for iodine from the external $\%$ text file. dnunL is set to 0.0075 based on work by $\mathrm{J}$. Codoni $\%$--D. Barnes

TRANSDATA = textread ('hfmodel_1storderNEQ_ms.txt', '\%f', 'headerlines', 1); TRANSDATA=reshape (TRANSDATA , 9 , length (TRANSDATA)/9);

$\mathrm{J}=\operatorname{TRANSDATA}(1,:)$;

$\mathrm{JP}=\operatorname{TRANSDATA}(2,:)$;

$\mathrm{V}=\operatorname{TRANSDATA}(3,:)$;

$\operatorname{VP}=\operatorname{TRANSDATA}(4,:)$;

FrequencyOfTransition $=$ TRANSDATA $(5,:) ; \%$ NUT

HyperFineDegeneracy $=$ TRANSDATA $(6,:) ; \%$ GHF

FrankCondonFactor $=\operatorname{TRANSDATA}(7,:) ; \% \mathrm{FCF}$ 


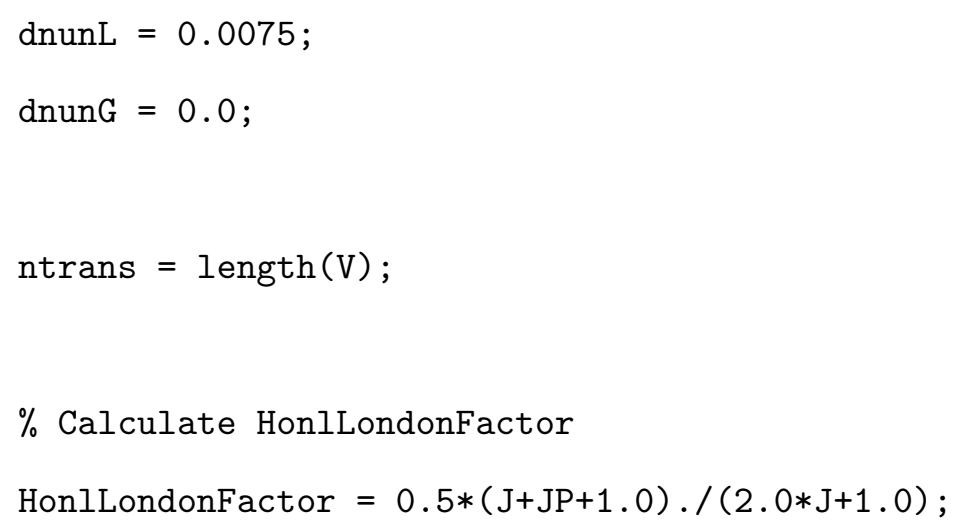




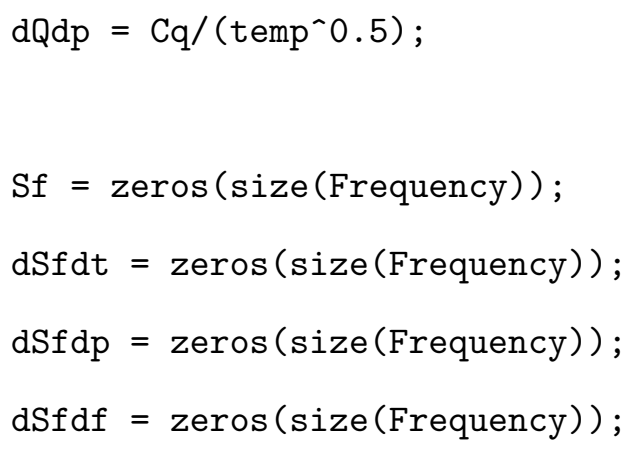




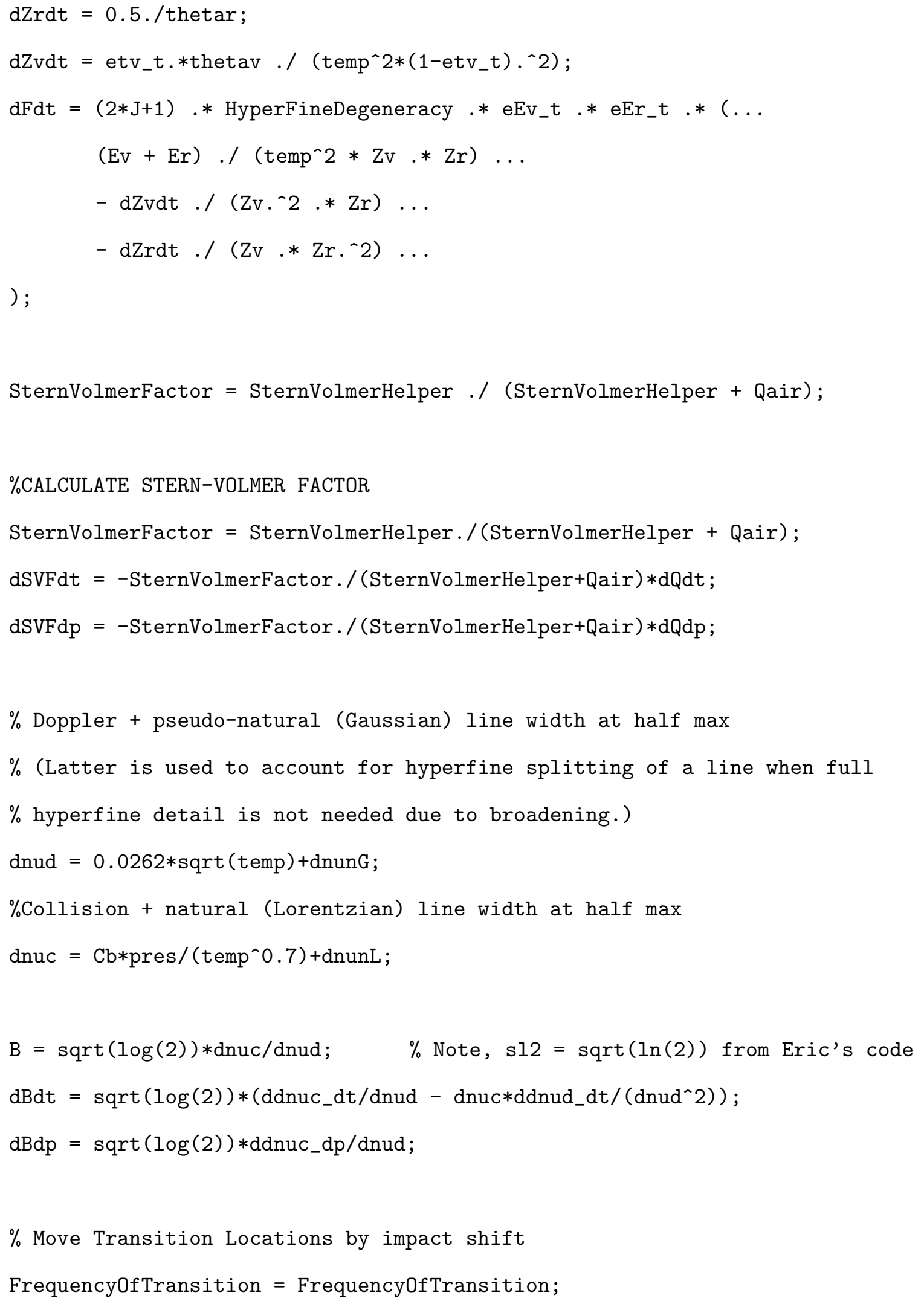




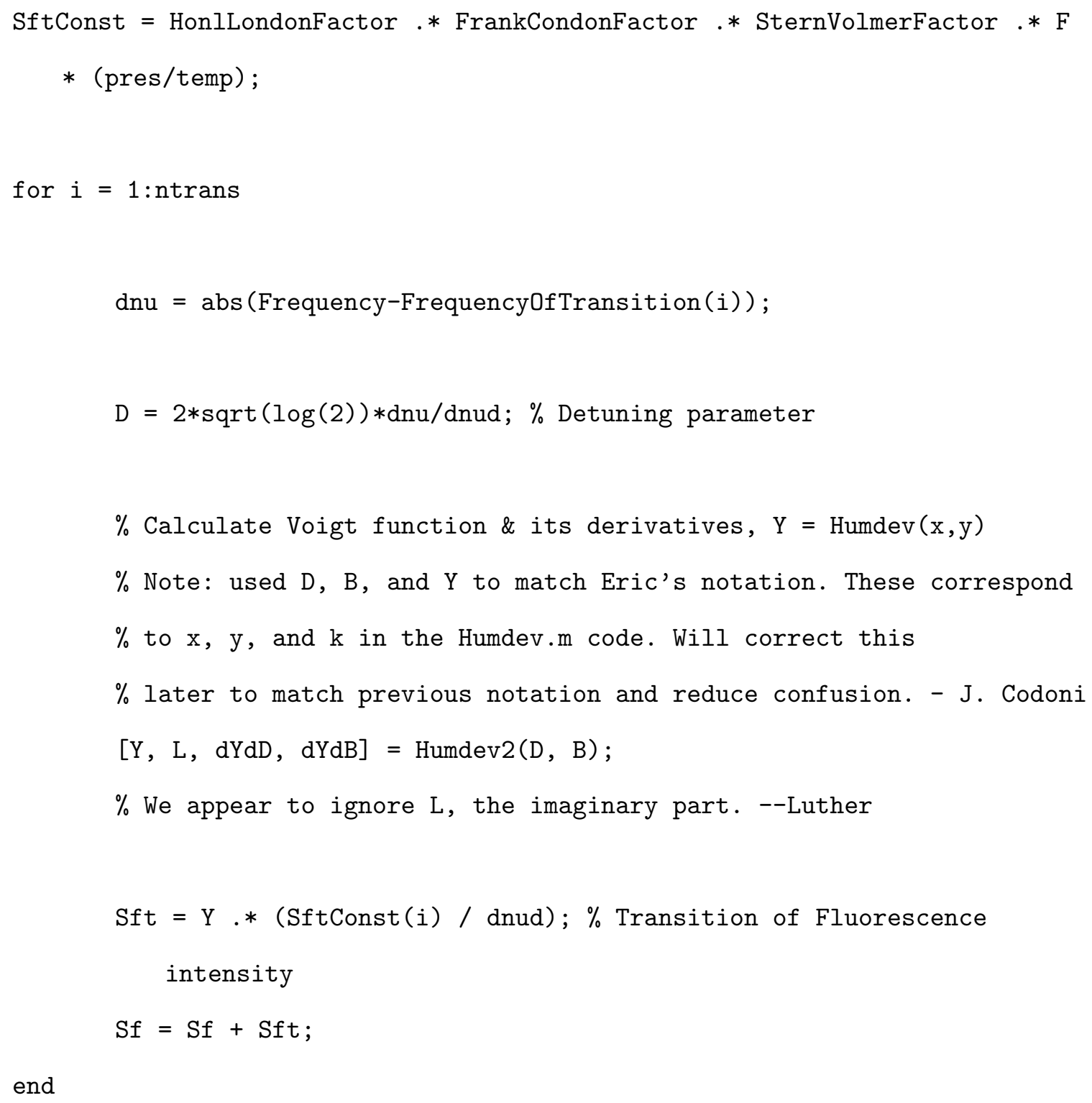

end

$\mathrm{Sf}=\mathrm{Sf}$

$\mathrm{Sf}=\mathrm{Sf} . / \max (\mathrm{Sf}) ; \%$ Commented out if spectra plots not normalized to 1

\section{Humdev2.m:}

function $[\mathrm{k}, \mathrm{L}, \mathrm{dkdx}, \mathrm{dkdy}]=\operatorname{Humdev} 2(\mathrm{x}, \mathrm{y})$

$\%$ HUMDEV Calculates the Faddeeva function and derivatives of the Voigt $\%$ function for $y>=0$ 
$\%$ Based on HUMDEV.C from Eric Cecil, his was a slightly modified code

$\%$ for the standard boxed Fortran 77 code called HUMDEV.for, which can

$\%$ be found here: http://www.atm.ox.ac.uk/user/wells/humdev.for

$\% \quad$ Adapted by Josh Codoni, 5/12/2011

$\% \quad \mathrm{k}$ is the Voigt function,

$\% \quad L$ is the imaginary part

$\% \mathrm{dk} / \mathrm{dx}$ is the partial derivative of the Voigt function

$\% \quad d k / d y$ is the partial derivative of the Voigt function

$\% \mathrm{n}$ is the number of points

$\% \quad \mathrm{x}$ is the input array in the $\mathrm{x}$-dir

$\% \quad y$ is the scalar input in the $y-\operatorname{dir}(y>=0)$

$\% \quad$ All outputs are column vectors

\% Edited by 20 Jun 2011 to accept $\mathrm{x}$ and y as vectors, have no loops, $\%$ and only compute $k$. The goal of this edit was speed. --Luther Tychonievich

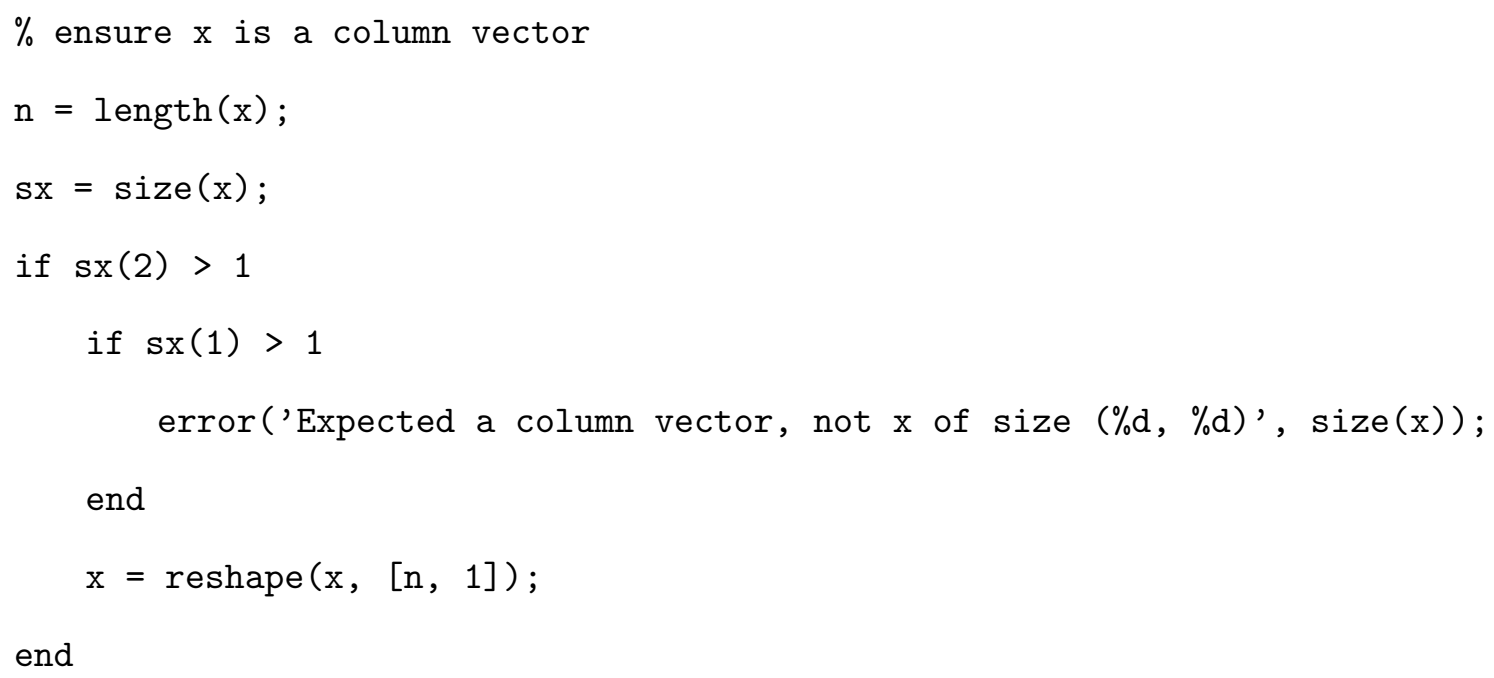




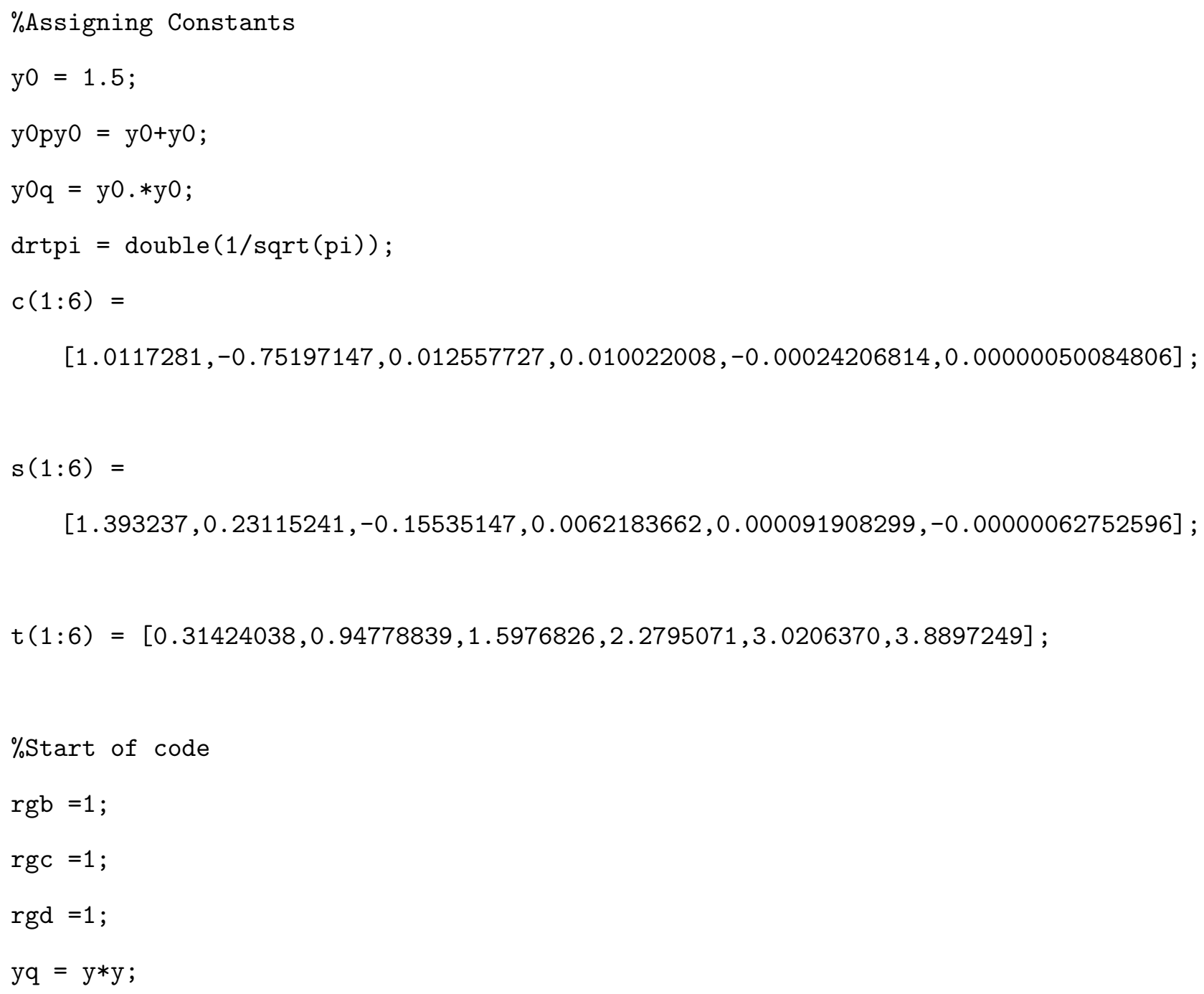


\%Loop over all points, taken out for now. When compared to values listed \%in R.J. Wells 1999 paper, Reference \#21 in Eric Cecil's Master's thesis, $\%$ in the table (page 40), results would match for $\mathrm{x} 1, \mathrm{y} 1, \mathrm{x} 2, \mathrm{y} 2, \mathrm{x} 4, \mathrm{y} 4$ values \%but produced strange results for $\mathrm{x} 3, \mathrm{y} 3$ values. Will attempt to correct $\%$ later.

\% replaced all loops with matrix operations. 20/Jun/2011 --Luther

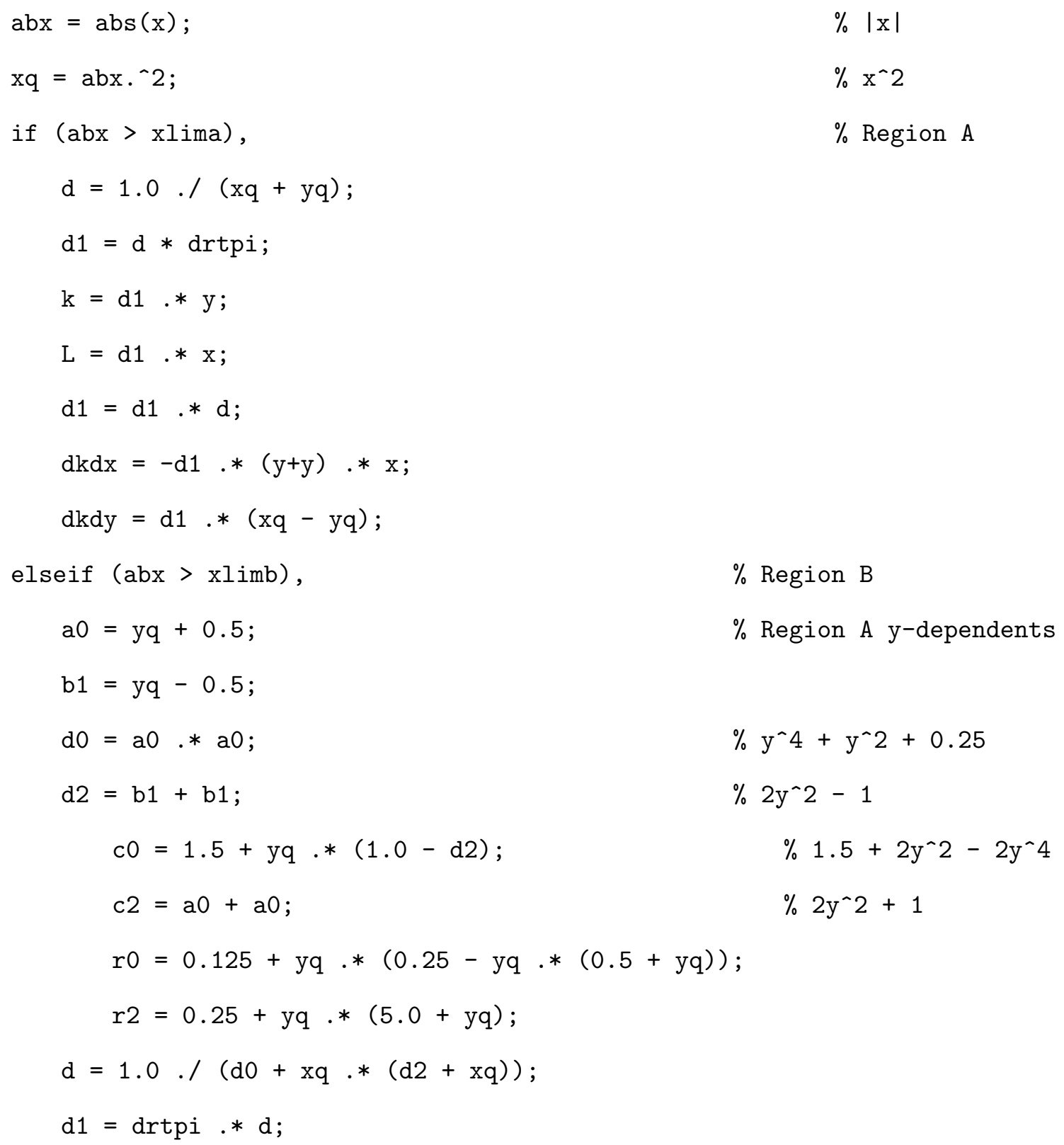




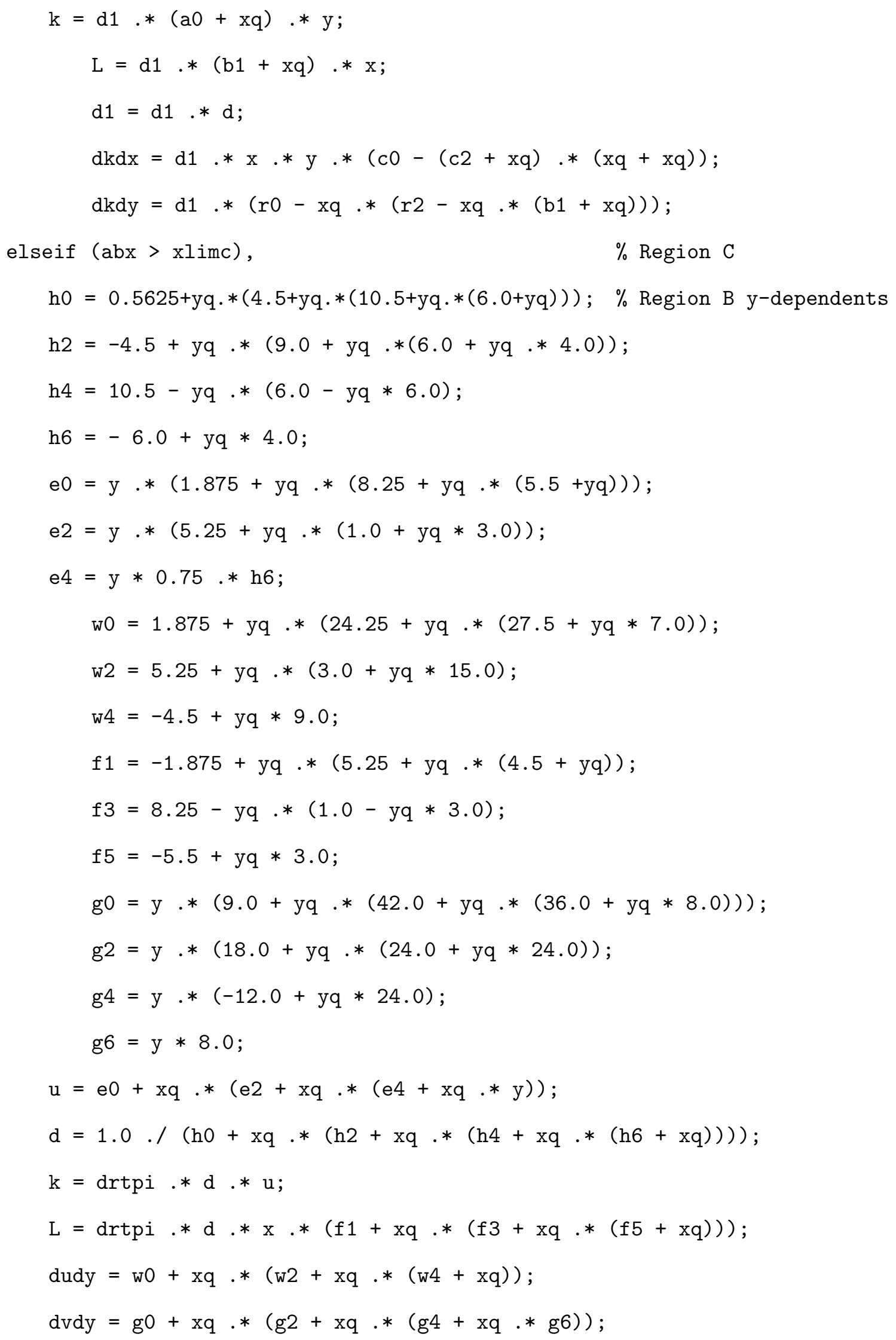




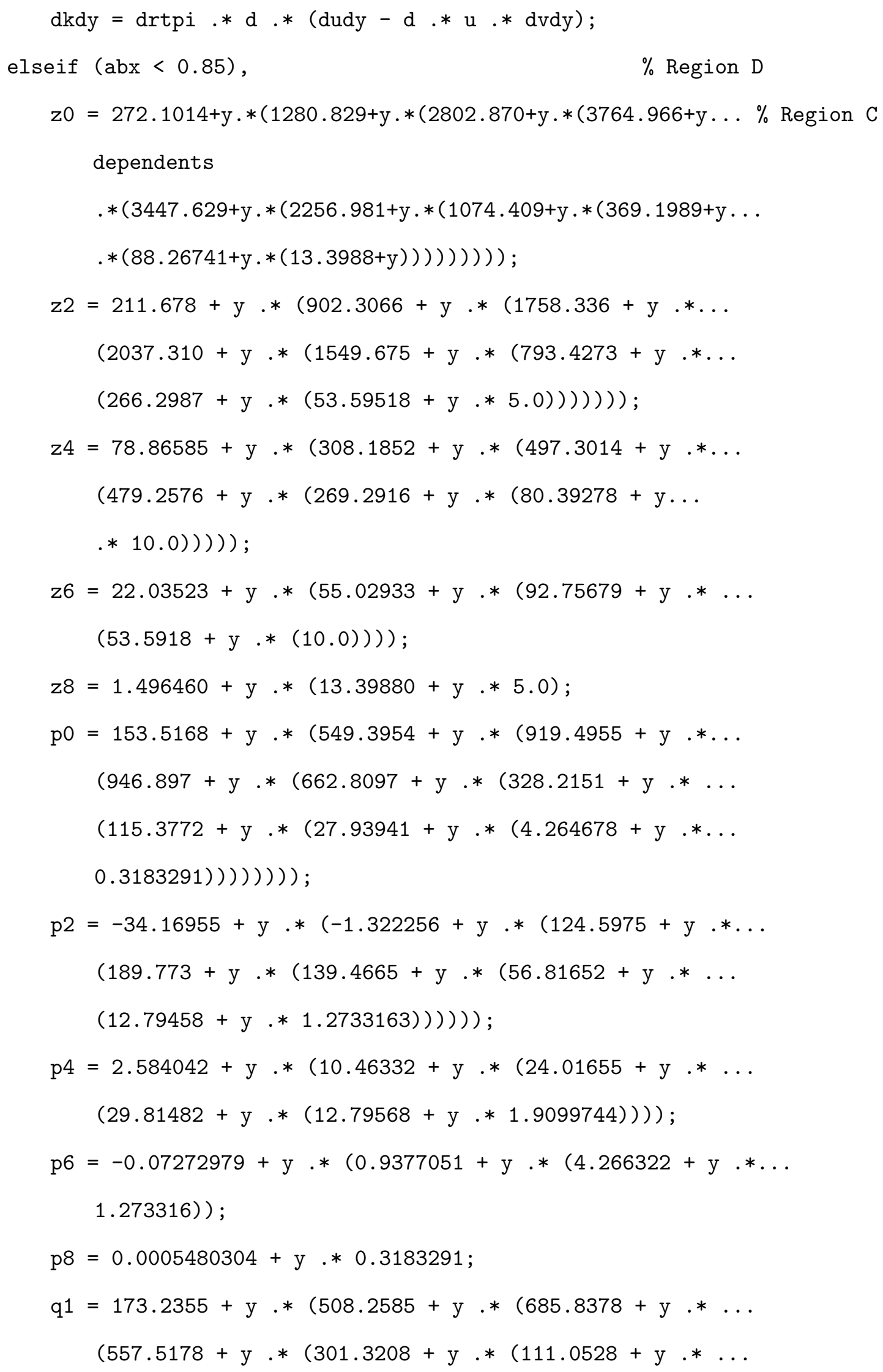




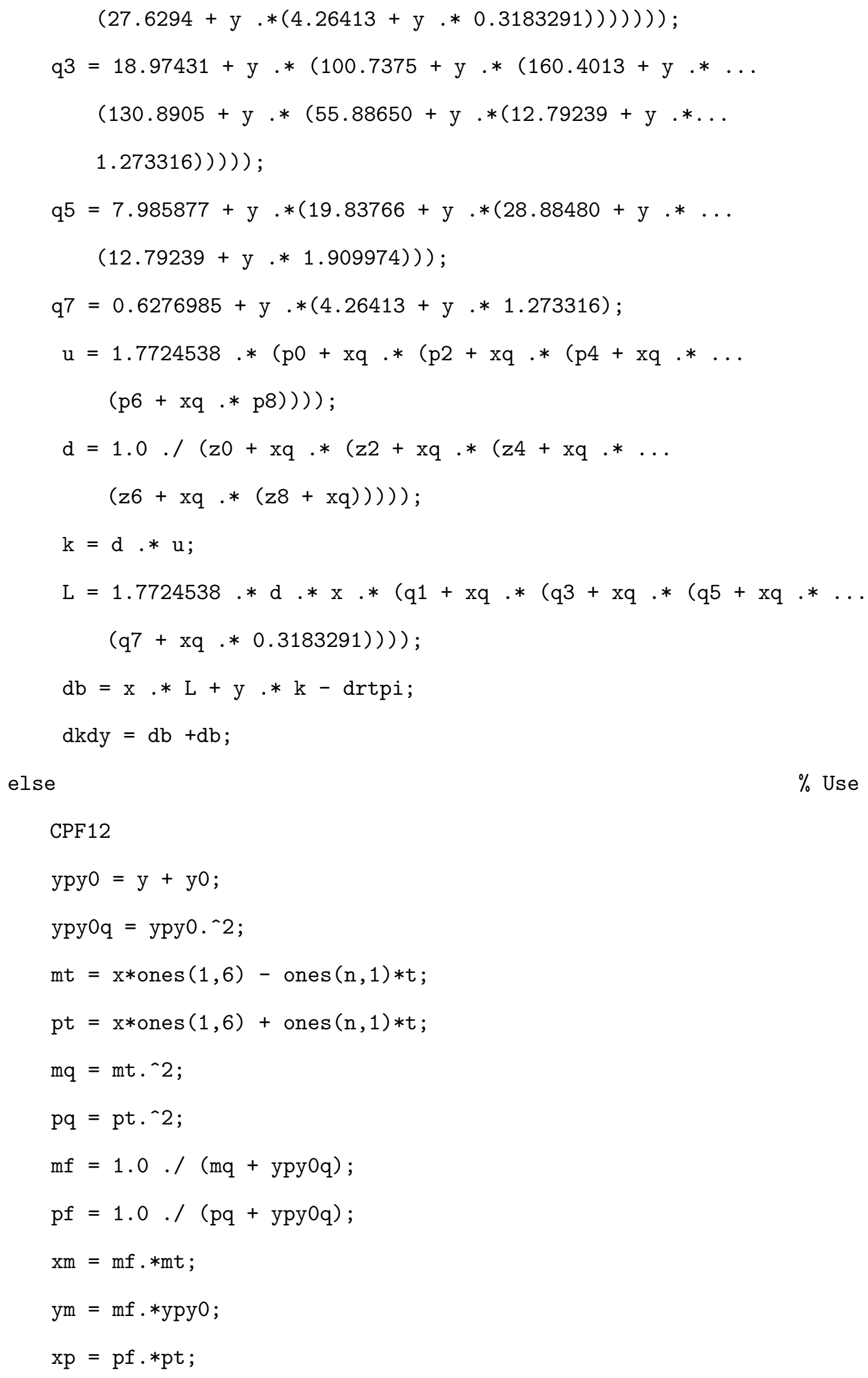


yp $=p f . * y p y 0 ;$

$L=(x m+x p) * c^{\prime}+(y m-y p) * s^{\prime} ;$

if $(\mathrm{abx}<=\mathrm{xlim} 4)$,

\% Humlicek CPF12 Region I

$$
\begin{aligned}
& \text { yf1 = ypy0 + ypy0; } \\
& \text { yf2 = ypy0q + ypy0q; } \\
& \text { dkdy = } 0.0 ;
\end{aligned}
$$

$m f q=m f \cdot{ }^{\wedge} 2$

$\mathrm{pfq}=\mathrm{pf} \cdot \wedge^{\wedge} 2$

$\mathrm{k}=(\mathrm{ym}+\mathrm{yp}) * \mathrm{c}^{\prime}-(\mathrm{xm}-\mathrm{xp}) * \mathrm{~s}^{\prime} ;$

$\mathrm{dkdy}=(\mathrm{mf}+\mathrm{pf}-\mathrm{yf} 2 *(\mathrm{mfq}+\mathrm{pfq})) * \mathrm{c}^{\prime}+\mathrm{yf} 1 *(\mathrm{mt} . * \mathrm{mfq}-\mathrm{pt} . * \mathrm{pfq}) * \mathrm{~s}^{\prime} ;$

else \% Humlicek CPF12 Region II

$$
\text { yp2y0 = y + y0py0; }
$$

$\mathrm{cl}=\operatorname{ones}(\mathrm{n}, 1) * \mathrm{c}$;

$\mathrm{sl}=\operatorname{ones}(\mathrm{n}, 1) * \mathrm{~s} ;$

$\mathrm{k}=(\mathrm{cl} . *(\mathrm{mq} . * \mathrm{mf}-\mathrm{y} 0 . * \mathrm{ym})+\mathrm{sl} \cdot * \mathrm{yp} 2 \mathrm{y} 0 . * \mathrm{xm}) . /(\mathrm{mq}+\mathrm{y} 0 \mathrm{q}) \ldots$

$+(\mathrm{cl} *(\mathrm{pq} . * \mathrm{pf}-\mathrm{y} 0 . * \mathrm{yp})-\mathrm{sl} *$ yp2y0.*xp) ./ (pq+y0q);

$\mathrm{k}=\mathrm{k} *$ ones $(6,1)$;

$\mathrm{k}=\mathrm{y} \cdot * \mathrm{k}+\exp (-\mathrm{xq}) ;$

$\mathrm{db}=\mathrm{x} \cdot * \mathrm{~L}+\mathrm{y} \cdot * \mathrm{k}-\mathrm{drtp} i$

$\mathrm{dkdy}=\mathrm{db} * 2 ;$

end

end

$\mathrm{db}=\mathrm{y} \cdot * \mathrm{~L}-\mathrm{x} \cdot * \mathrm{k} ;$

$\mathrm{dkdx}=2 * \mathrm{db}$ 


\section{Appendix E}

\section{Additional Hyperfine Spectrum Jet Core Comparisons}

This appendix contains additional comparisons of the resolution of the iodine hyperfine spectrum in the core fluorescence signal of a single jet. This is additional information and analyses performed in the manner presented in Chapter 5 of this research, and acts as additional proof of the resultion of the iodine hyperfine spectrum in the jet core fluorescence signal.

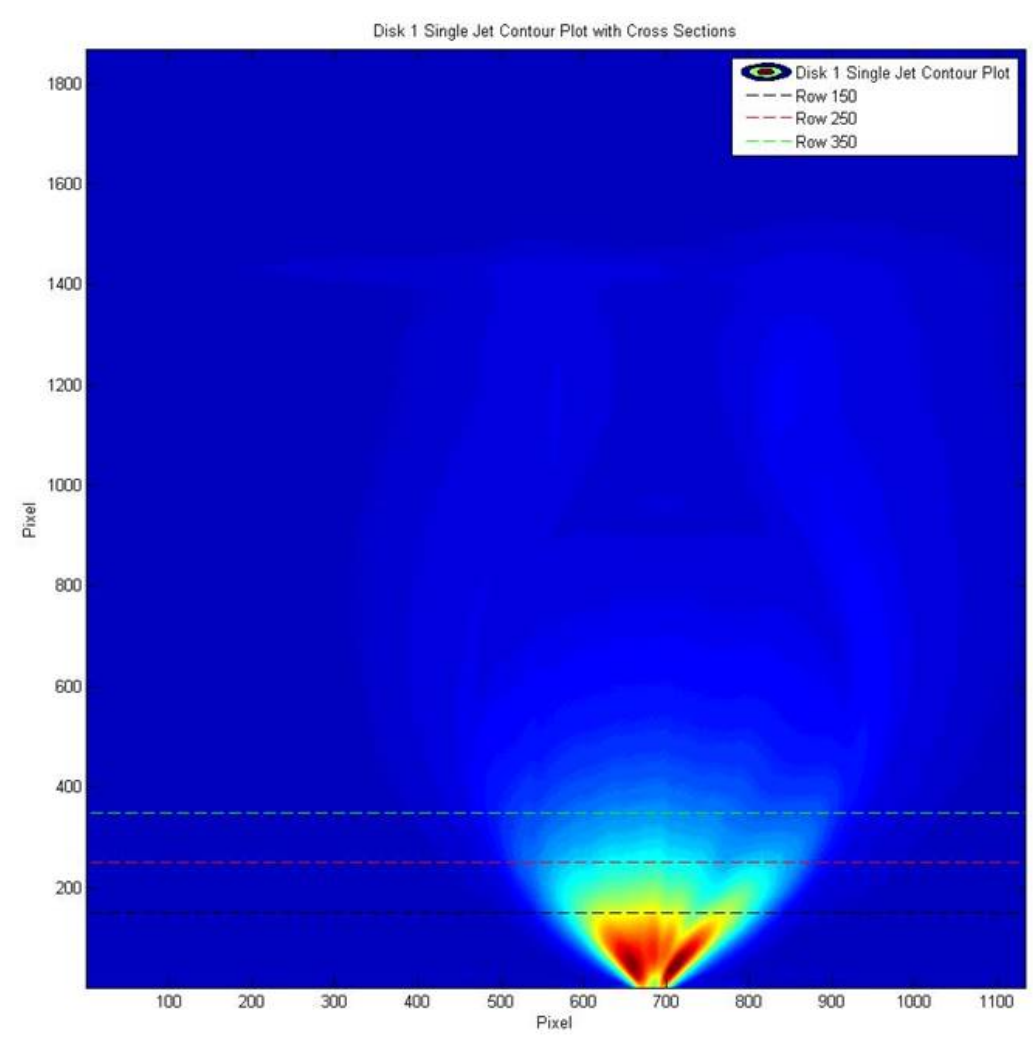

Figure E.1: Single jet contour plot, $P_{\text {ratio }}=19310$. 

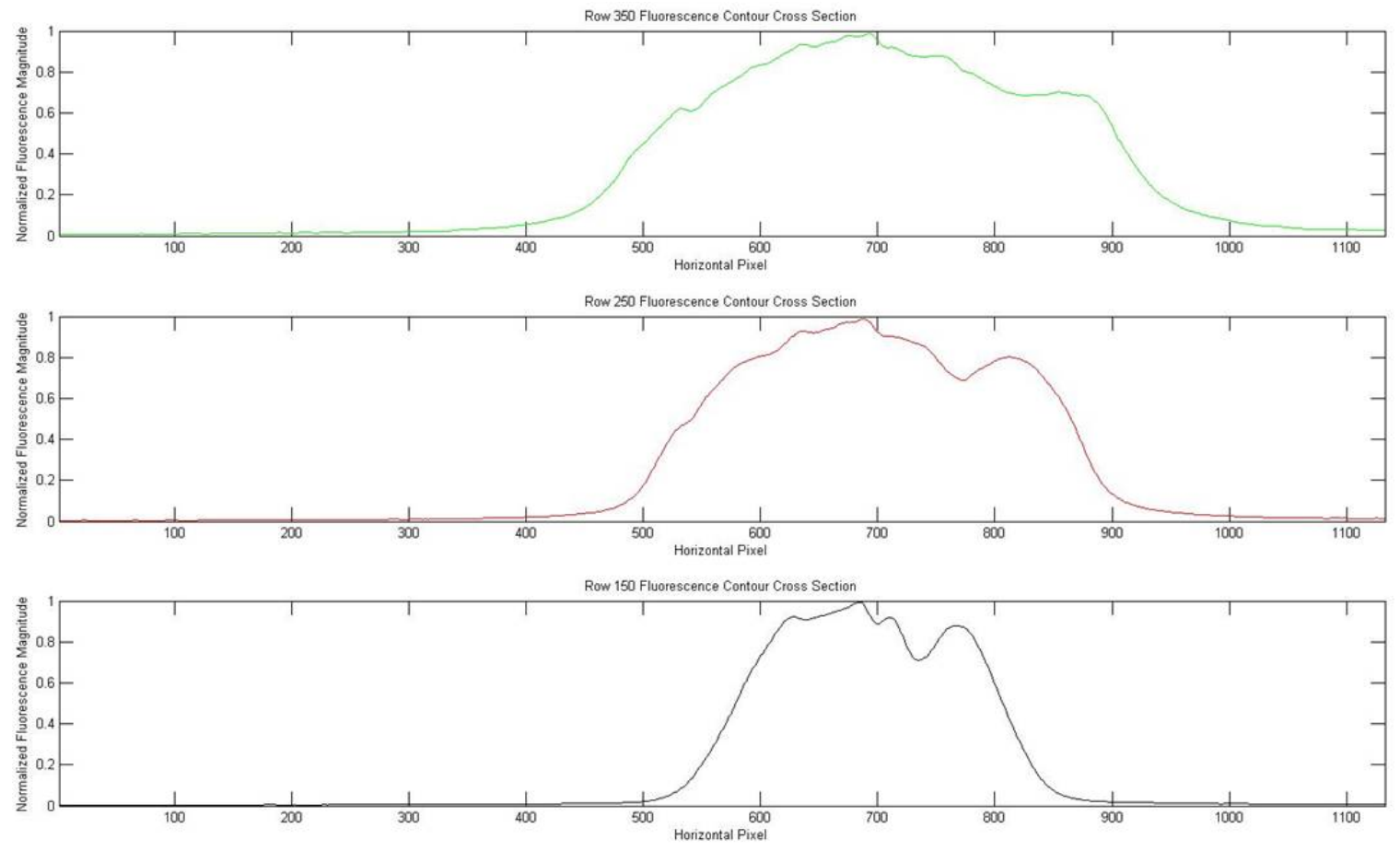

Figure E.2: Overlay of Three Fluorescence Horizontal Cross-sections. 


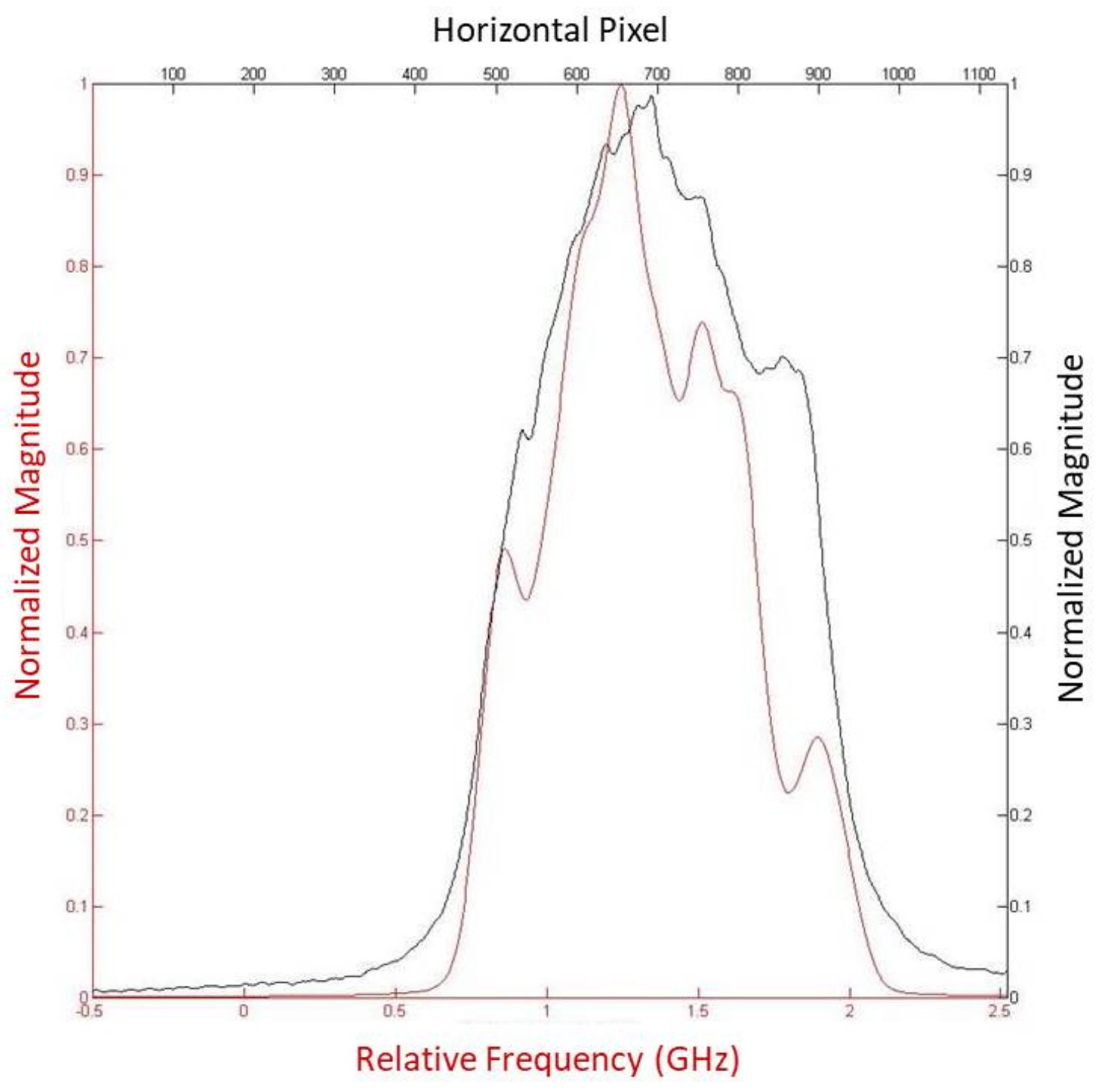

Figure E.3: Row 350, Comparison of Predicted Spectrum \& Fluorescence Signal. 


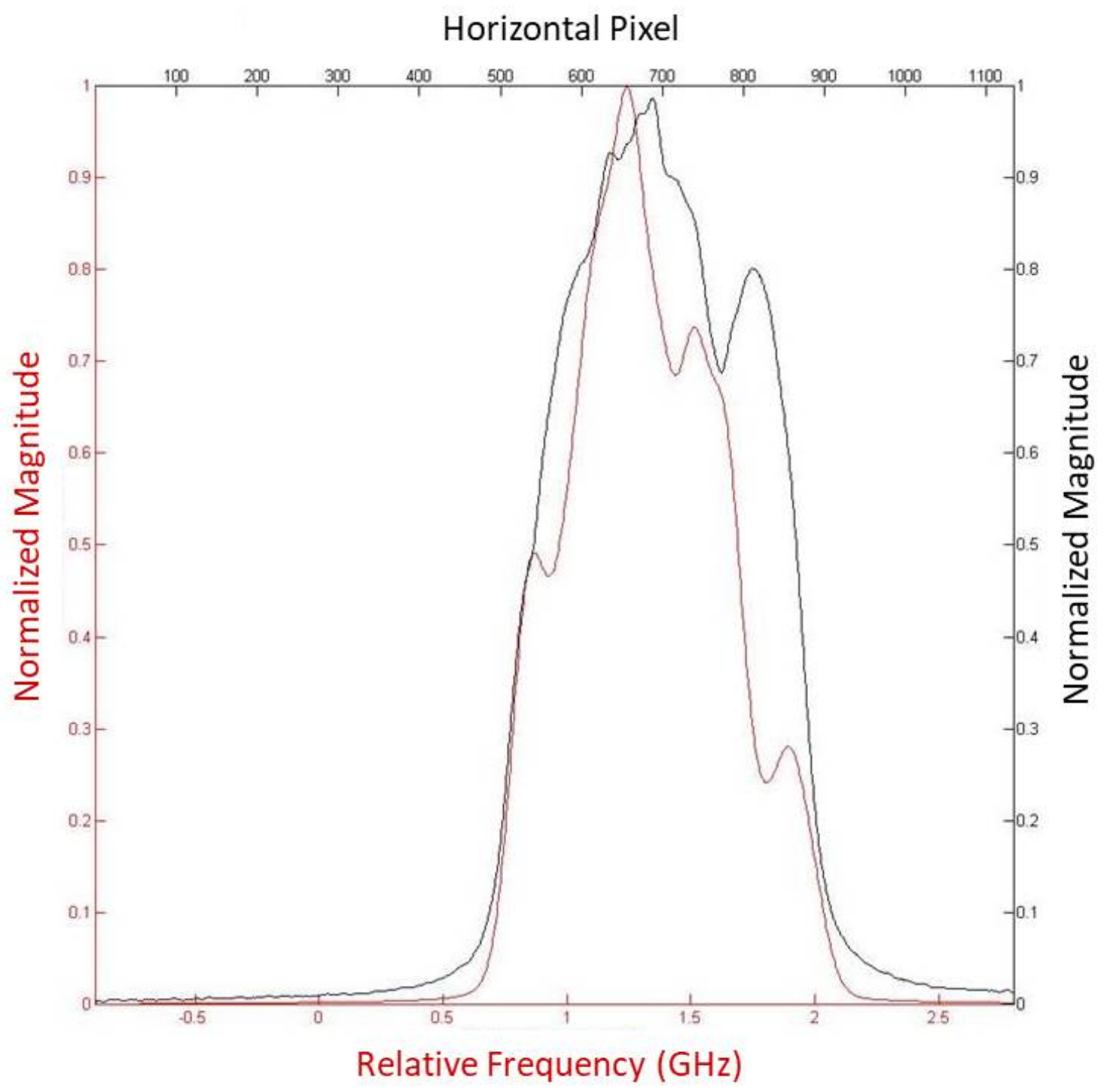

Figure E.4: Row 250, Comparison of Predicted Spectrum \& Fluorescence Signal. 


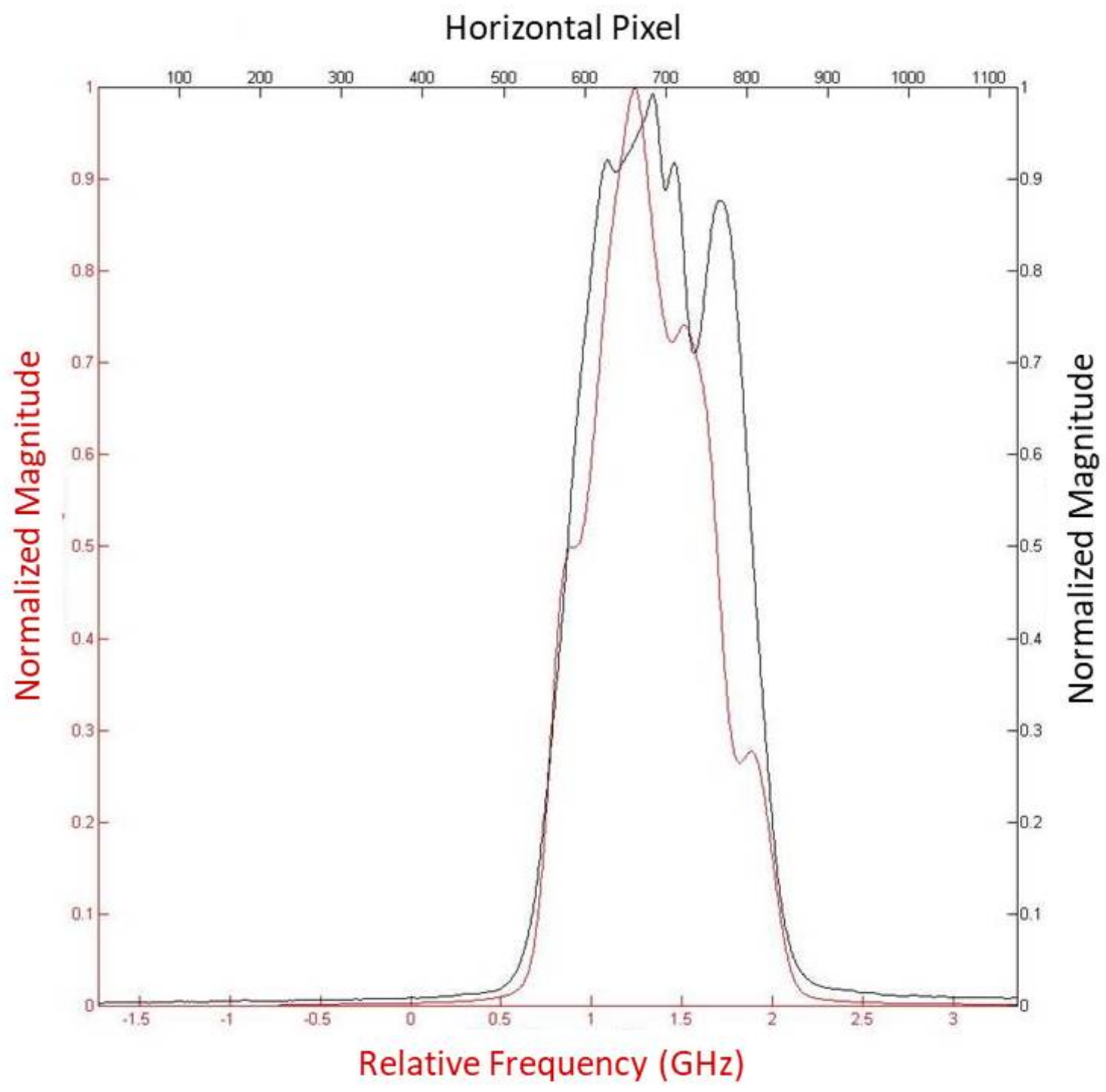

Figure E.5: Row 150, Comparison of Predicted Spectrum \& Fluorescence Signal. 


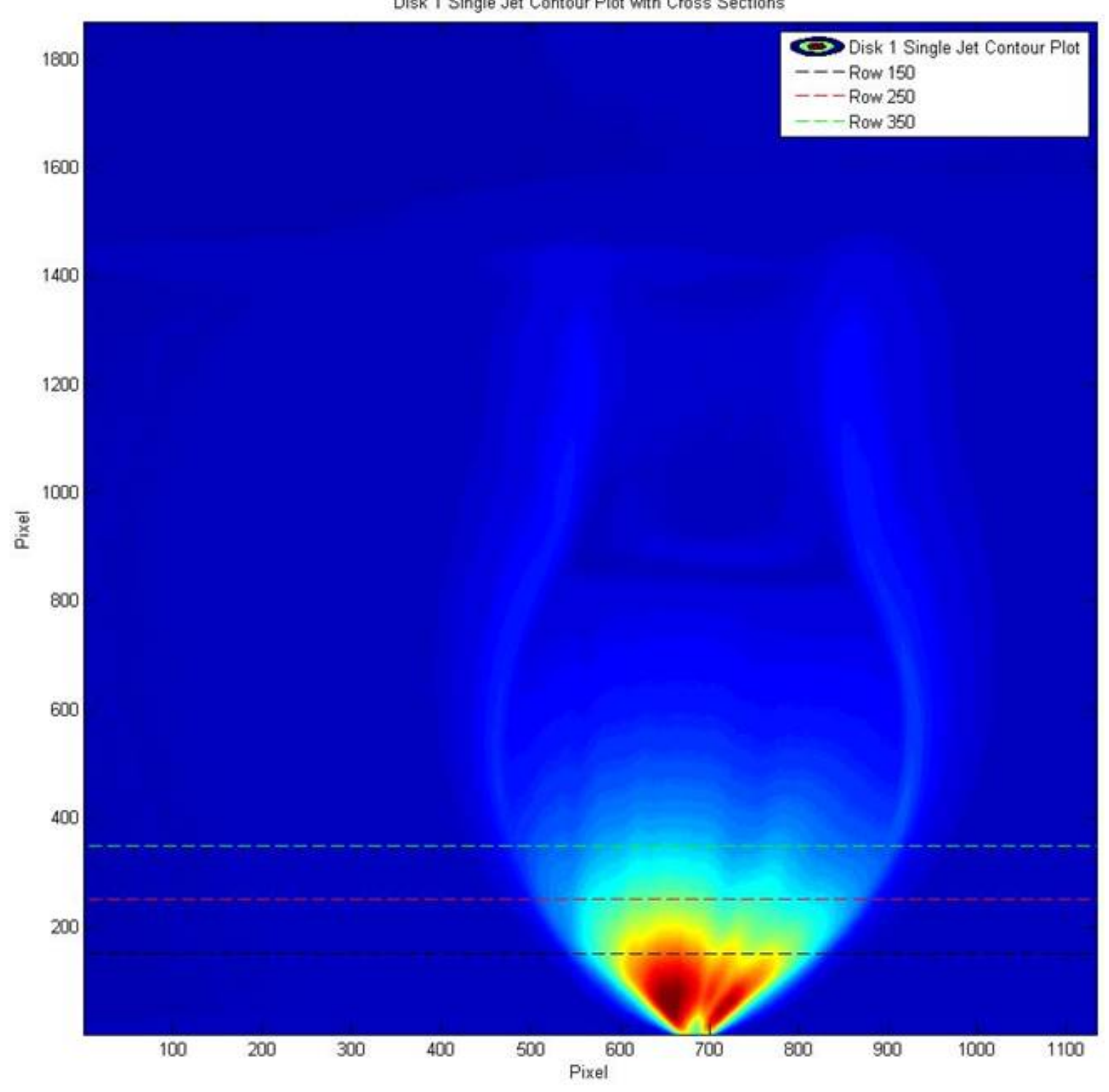

Figure E.6: Single jet contour plot, $P_{\text {ratio }}=18170$. 

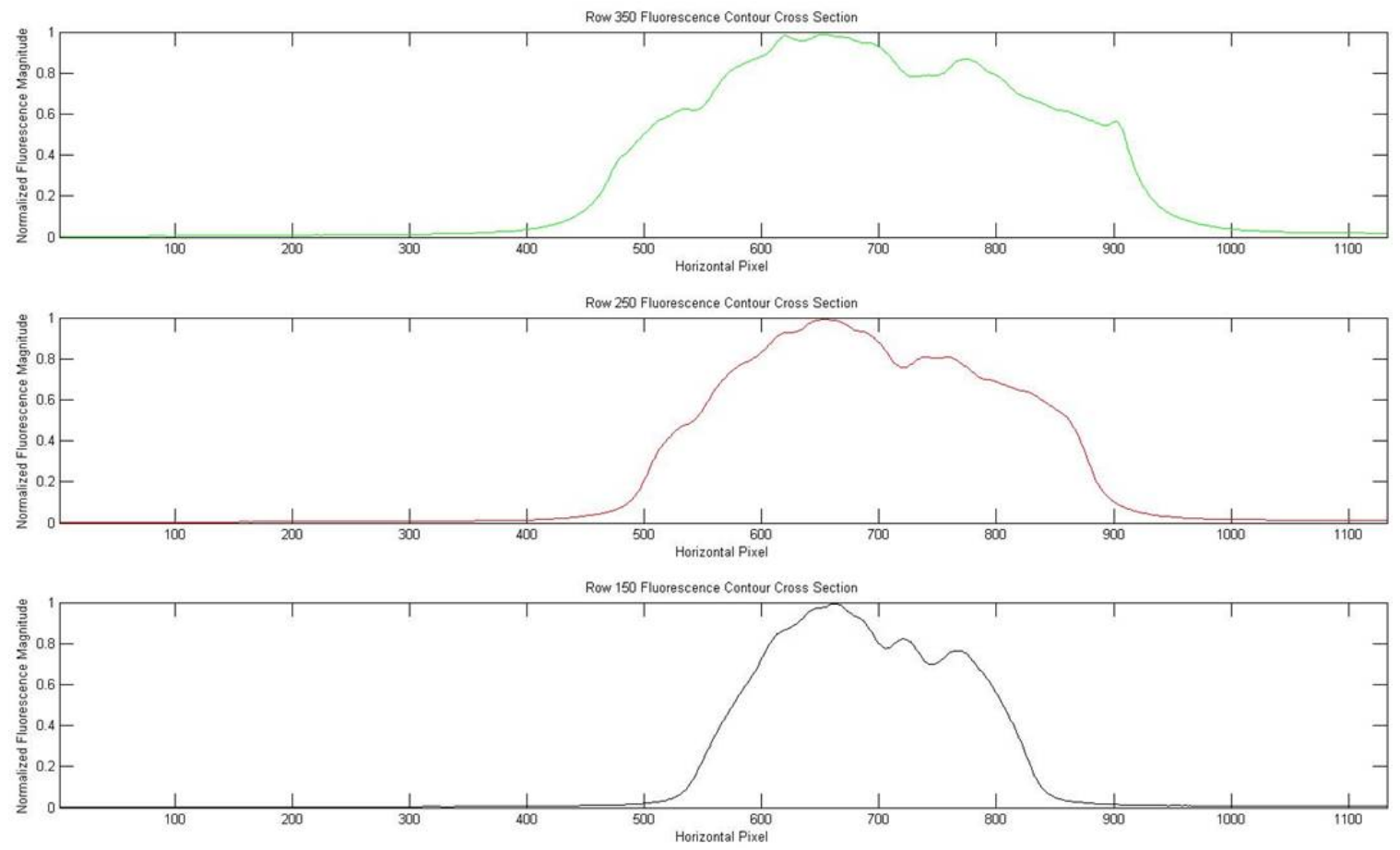

Figure E.7: Overlay of Three Fluorescence Horizontal Cross-sections. 


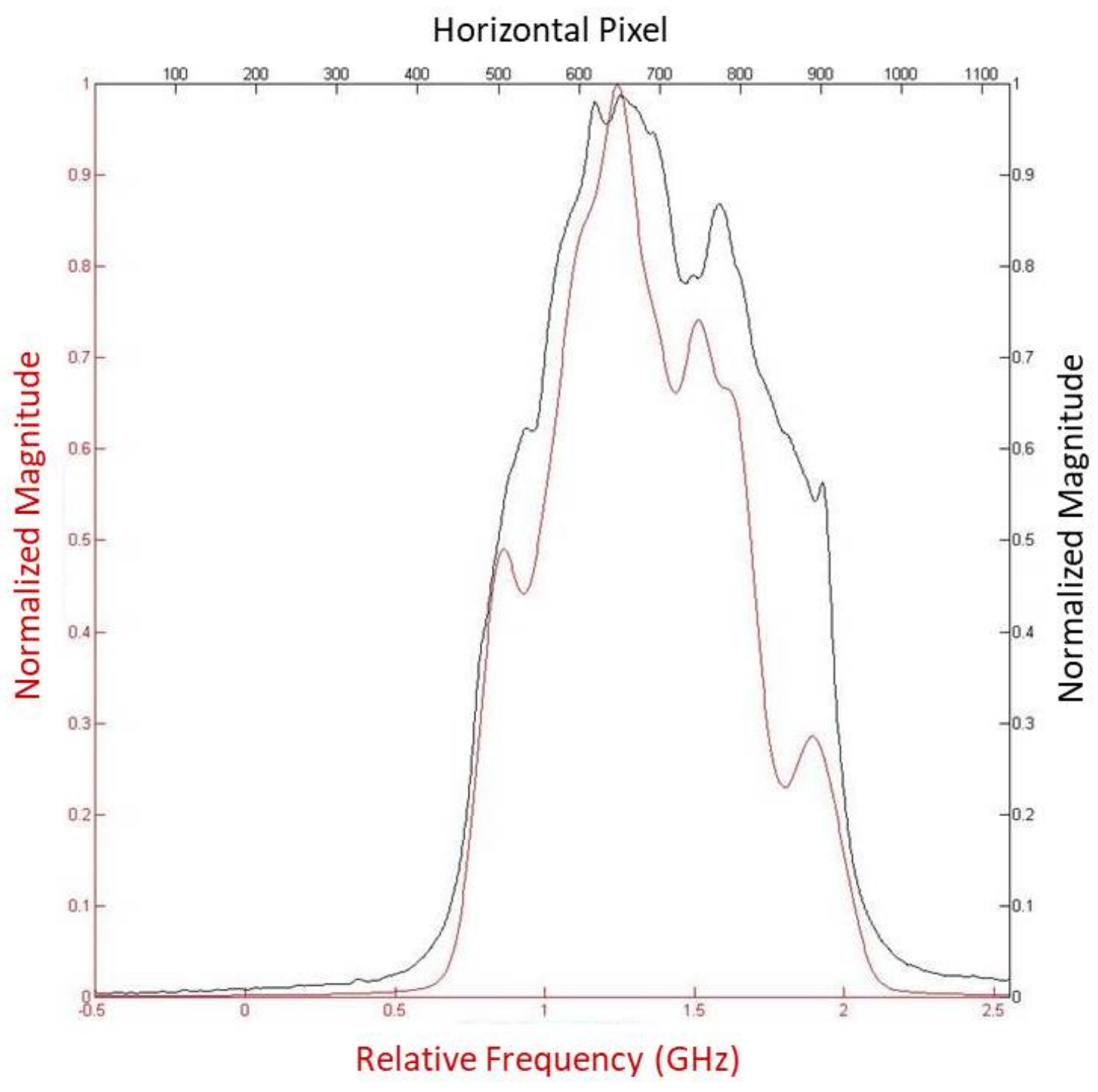

Figure E.8: Row 350, Comparison of Predicted Spectrum \& Fluorescence Signal. 


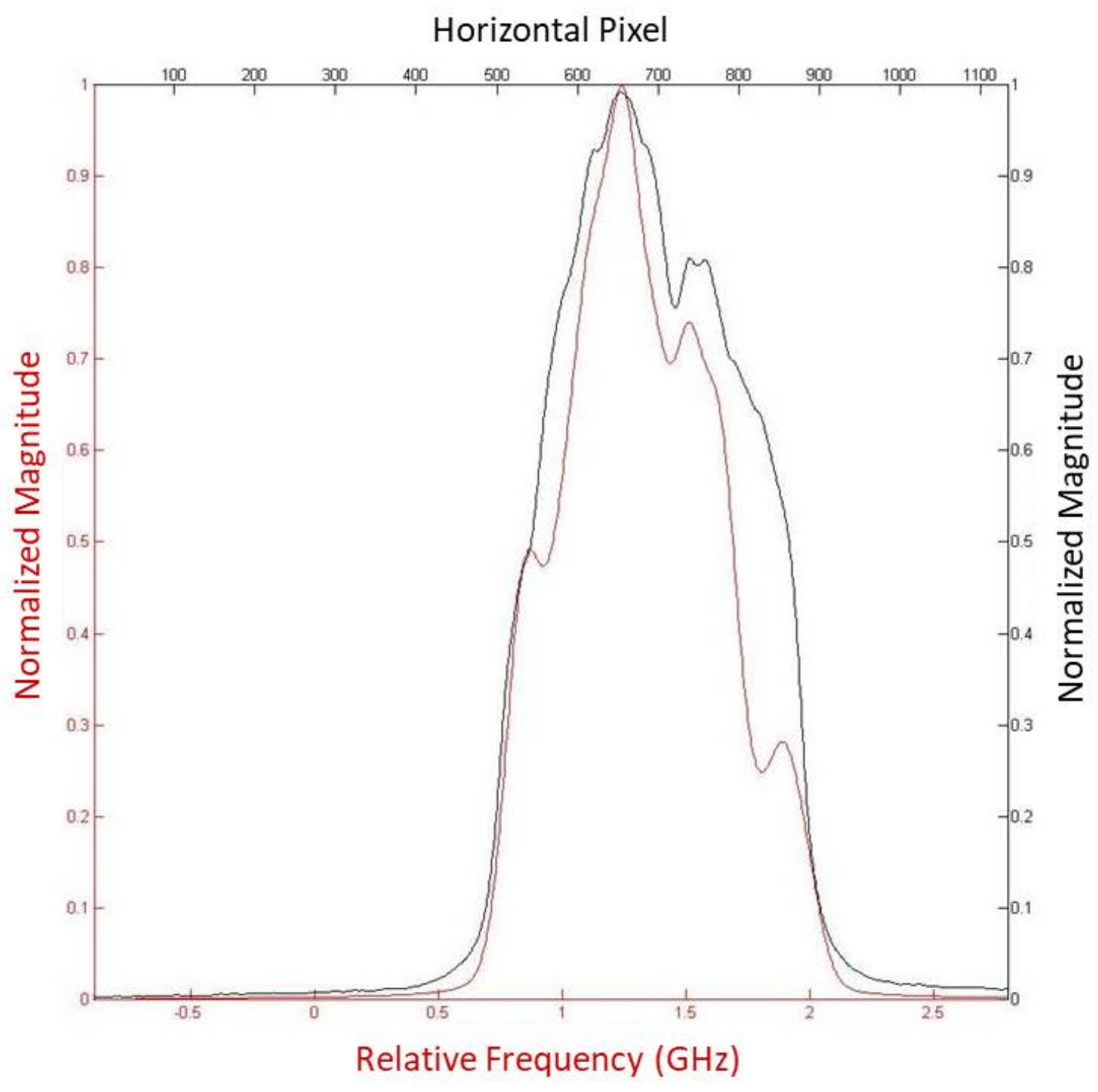

Figure E.9: Row 250, Comparison of Predicted Spectrum \& Fluorescence Signal. 


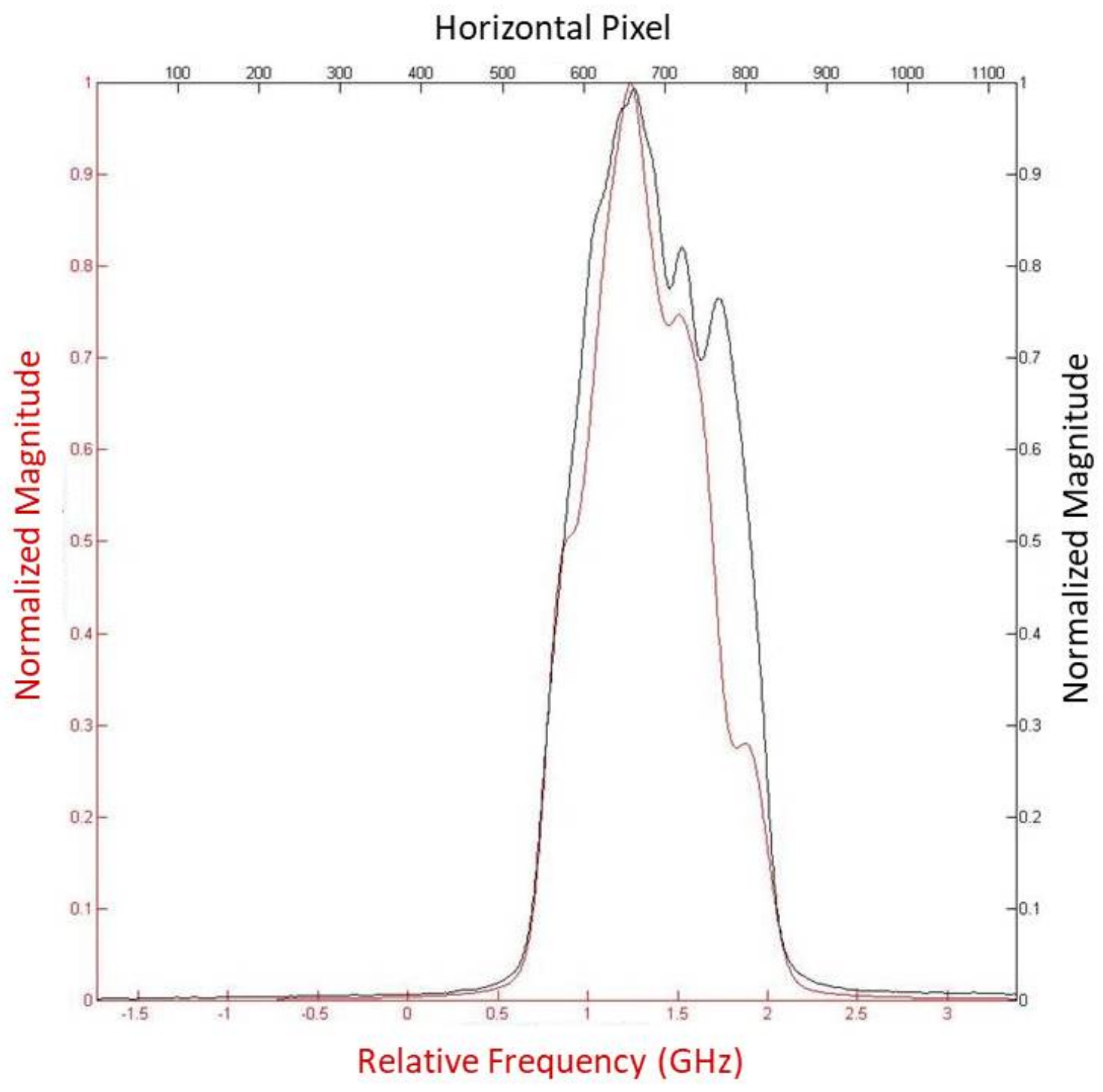

Figure E.10: Row 150, Comparison of Predicted Spectrum \& Fluorescence Signal. 


\section{REFERENCES}

[1] K. Yamanaka. Rendezvous strategy of the japanese logistics support vehicle to the international space station. In Spacecraft Guidance, Navigation and Control Systems, volume 381, page 103, 1997.

[2] Forrest E. Lumpkin III, Jeremiah Marichalar, and Benedicte D. Stewart. High fidelity simulations of plume impingement to the international space station. 33rd JANNAF Exhaust Plume aud Signatures, 2012.

[3] T. C. Adamson Jr. On the structure of jets from highly underexpanded nozzles into still air. Journal of the Aerospace Sciences, 26(1), 1959.

[4] D. J. Carlson and C. H. Lewis. Normal shock location in underexpanded gas and gas-particle jets. AIAA Journal, 2(4), 1964.

[5] I. Shih Chang and W.L. Chow. Mach disk from underexpanded axisymmetric nozzle flow. AIAA journal, 12(8):1079-1082, 1974.

[6] S. Crist, D. R. Glass, and P. M. Sherman. Study of the highly underexpanded sonic jet. AIAA Journal, 4(1), 1966.

[7] B. C. R. Ewan and K. Moodie. Structure and velocity measurements in underexpanded jets. Combustion Science and Technology, 45(5-6), 1986.

[8] Michael Abbett. Mach disk in underexpanded exhaust plumes. AIAA Journal, 9(3):512-514, 1971.

[9] A.L. Addy. Effects of axisymmetric sonic nozzle geometry on mach disk characteristics. AIAA Journal, 19(1):121-122, 1981.

[10] J.M. Foster, B.H. Wilde, P.A. Rosen, T.S. Perry, M. Fell, M.J. Edwards, B.F. Lasinski, R.E. Turner, and M.L. Gittings. Supersonic jet and shock interactions. Physics of Plasmas, 9(5):2251-2263, 2002.

[11] Yiannis Andreopoulos, Juan H. Agui, and George Briassulis. Shock waveturbulence interactions. Annual Review of Fluid Mechanics, 32(1):309-345, 2000.

[12] H.K. Cheng. Perspectives on hypersonic viscous flow research. Annual review of fluid mechanics, 25(1):455-484, 1993.

[13] Jean M. Delery. Experimental investigation of turbulence properties in transonic shock/boundary-layer interactions. AIAA journal, 21(2):180-185, 1983. 
[14] R.W. Wlezien. Nozzle geometry effects on supersonic jet interaction. AIAA journal, 27(10):1361-1367, 1989.

[15] J. Panda and R. G. Seasholtz. Measurement of shock structure and shockvortex interaction in underexpanded jets using rayleigh scattering. Physics of Fluids, 11(12), 1999.

[16] F. S. Alvi, J. A. Ladd, and W. Bower. Experimental and computational investigation of supersonic impinging jets. AIAA Journal, 40(4), 2002.

[17] P. J. Lamont and B. L. Hunt. The impingement of underexpanded, axisymmetric jets on perpendicular and inclined flat plates. Journal of Fluid Mechanics, 100(3), 1980 .

[18] Yusuke Nakai, Nobuyoshi Fujimatsu, and Kozo Fujii. Experimental study of underexpanded supersonic jet impingement on an inclined flat plate. AIAA Journal, 44(11), 2006.

[19] S.G. Jennings. The mean free path in air. Journal of Aerosol Science, 19(2):159$166,1988$.

[20] Dan Dessau. Vacuum science techniques and applications. University Lecture, 2007.

[21] N.M. Laurendeau. Statistical Thermodynamics: Fundamentals and Applications. Cambridge University Press, 2005.

[22] Earle Hesse Kennard. Kinetic theory of gases: with an introduction to statistical mechanics. McGraw-Hill, 1938.

[23] Walter Guido Vincenti and Charles H. Kruger. Introduction to physical gas dynamics. Krieger, 1967.

[24] P.L. Owen and C.K. Thornhill. The flow in an axially symmetric supersonic jet from a nearly sonic orifice into a vacuum. Ministry of Supply, Armament Research Establishment, Physical Research Division, 1948.

[25] Eugene S. Love, Carl E. Grigsby, Louise P. Lee, and Mildred J. Woodling. Experimental and theoretical studies of axisymmetric free jets. NASA Technical Report NASA-TR-R-6, 1959.

[26] J.D. Anderson. Modern Compressible Flow: With Historical Perspective. Aeronautical and Aerospace Engineering Series. McGraw-Hill Education, 2003.

[27] S. K. Godunov. Finite-difference method for the numerical computation of discontinuous solutions of the equations of fluid dynamics. Matematicheskii Sborni, 47(1):271-306, 1959.

[28] Frank A. Albini. Approximate computation of underexpanded jet structure. AIAA Journal, 3(8):1535-1537, 1965. 
[29] Frederick P. Boynton. Highly underexpanded jet structure-exact and approximate calculations. AIAA Journal, 5(9):1703-1704, 1967.

[30] E.K. Latvala and T.P. Anderson. Studies of the spreading of rocket exhaust jets at high altitudes. Planetary and Space Science, 4:77-91, 1961.

[31] Richard M. Beam and Robert F. Warming. An implicit finite-difference algorithm for hyperbolic systems in conservation-law form. Journal of computational physics, 22(1):87-110, 1976.

[32] Richard M Beam and RoF Warming. An implicit factored scheme for the compressible navier-stokes equations. AIAA journal, 16(4):393-402, 1978.

[33] W.R. Briley and H. McDonald. Solution of the multidimensional compressible navier-stokes equations by a generalized implicit method. Journal of Computational Physics, 24(4):372-397, 1977.

[34] Tannehill John C. Pletcher, Richard H. and Dale Anderson. Computational Fluid Mechanics and Heat Transfer, Second Edition. CRC Press, 1997.

[35] Er-yun Chen, Gui-gao Le, Gai-ping Zhao, et al. Numerical simulation of highly underexpanded axisymmetric jet with runge-kutta discontinuous galerkin finite element method. Journal of Hydrodynamics, Ser. B, 20(5):617-623, 2008.

[36] Antony Jameson, Wolfgang Schmidt, and Eli Turkel. Numerical solution of the euler equations by finite volume methods using runge kutta time stepping schemes. In 14th fluid and plasma dynamics conference, page 1259, 1981.

[37] Malsur Dharavath and Debasis Chakraborty. Numerical simulation of supersonic jet impingement on inclined plate. Defence Science Journal, 63(4):355-362, 2013.

[38] G. A. Bird. Molecular Gas Dynamics and the Direct Simulation of Gas Flows. Clarendon Press, 1994.

[39] Iain D. Boyd. Direct simulation monte carlo for atmospheric entry. 2. code development and application results. Technical report, DTIC Document, 2009.

[40] J. C. McDaniel. Investigation of laser-induced iodine fluorescence for the measurement of density in compressible flows. PhD thesis, Stanford University, 5 1981.

[41] James C. McDaniel, B. Hiller, and Ronald K. Hanson. Simultaneous multiplepoint velocity measurements using laser-induced iodine fluorescence. Optics Letters, 8(1):51-53, 1983.

[42] William Letko. Loads induced on a flat plate at a Mach number of 4.5 with a sonic or supersonic jet exhausting normal to the surface. National Aeronautics and Space Administration, 1963. 
[43] Mara M. Orescanin, David Prisco, Joanna M. Austin, and Susan W. Kieffer. Flow of supersonic jets across flat plates: Implications for ground-level flow from volcanic blasts. Journal of Geophysical Research: Solid Earth, 119(4):2976-2987, 2014.

[44] Huaping Zhen, Zhenxun Gao, and Chunhian Lee. Numerical investigation on jet interaction with a compression ramp. Chinese Journal of Aeronautics, 26(4):898908, 2013.

[45] B. Hiller and R.K. Hanson. Properties of the iodine molecule relevant to laserinduced fluorescence experiments in gas flows. Experiments in fluids, 10(1):1-11, 1990.

[46] James Donohue. Experimental and numerical study of ramp injectors for supersonic fuel/air mixing. PhD thesis, University of Virginia, 11995.

[47] Douglas G. Fletcher. Spatially-resolved, nonintrusive measurements in a nonreacting scramjet combustor using laser-induced iodine fluorescence. $\mathrm{PhD}$ thesis, University of Virginia, 11989.

[48] R. J. Hartfield. Planar Measurement of Flow Field Parameters in Nonreacting Supersonic Flows with Laser-induced Iodine Fluorescence. PhD thesis, University of Virginia, 51991.

[49] Eric Cecil. Velocity Field Measurements in Rarefied, Hypersonic Flows of Nitrogen Using Laser-Induced Fluorescence of Iodine. PhD thesis, University of Virginia, 122011.

[50] Erin M. Reed. Planar Laser Induced Iodine Fluorescence for the Investigation of the Aerodynamics of Reaction Control System Jets on Mars-Entry Aeroshells. PhD thesis, University of Virginia, 122013.

[51] Joshua Codoni. Investigation of Hypersonic Retropropulsion Using Planar LaserInduced Iodine Fluorescence. PhD thesis, University of Virginia, 52014.

[52] A.K. Mohamed, J. Bonnet, S. Larigaldie, T. Pot, J. Soutadé, and B. Diop. Electron beam fluorescence in hypersonic facilities. AerospaceLab, 1(1):p-1, 2009.

[53] Eric Phillip Muntz. The electron beam fluorescence technique. Technical report, DTIC Document, 1968.

[54] James C. McDaniel, Joshua R. Codoni, Erin M. Reed, Hicham Alkandry, and Iain D. Boyd. Propulsion deceleration studies using planar laser-induced iodine fluorescence and computational fluid dynamics. Journal of Spacecraft and Rockets, 50(4):771-780, 2013.

[55] G. Herzberg. Molecular spectra and molecular structure. Number v. 1 in PrenticeHall physics series ; . U. Condon, ed. Van Nostrand, 1950. 
[56] J. Humlíček. An efficient method for evaluation of the complex probability function: the voigt function and its derivatives. Journal of Quantitative Spectroscopy and Radiative Transfer, 21(4):309-313, 1979.

[57] Ira N. Levine et al. Molecular spectroscopy. Wiley, 1975.

[58] Roy J. Hartfield, John D. Abbitt, and James C. McDaniel. Injectant molefraction imaging in compressible mixing flows using planar laser-induced iodine fluorescence. Optics letters, 14(16):850-852, 1989.

[59] Spectra Physics Lasers. Spectra Physics BeamLok 2060 and 2080 Ion Lasers User's Manual, 1997.

[60] H. Ashkenas and F.S. Sherman. The structure and utilization of suerpsonic free jets in low density wind tunnels. Rarefied Gas Dynamics, 2, 1966.

[61] Daniel Rimkus and Norman A. Baily. Quantum noise in detectors. Medical physics, 10(4):470-471, 1983.

[62] John Taylor. Introduction to error analysis, the study of uncertainties in physical measurements. University Science Books, 1997. 